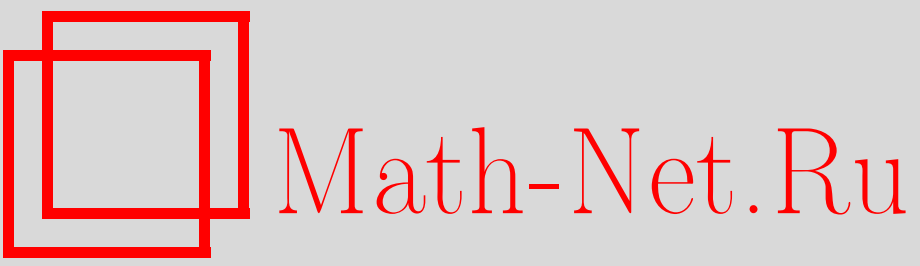

С. П. Суетин, Аппроксимации Паде и эффективное аналитическое продолжение степенного ряда, УМH, 2002, том 57, выпуск 1, 45-142

DOI: https://doi.org/10.4213/rm475

Использование Общероссийского математического портала Math-Net.Ru подразумевает, что вы прочитали и согласны с пользовательским соглашением

http://www.mathnet.ru/rus/agreement

Параметры загрузки:

IP: 52.205.19.152

26 апреля 2023 г., 13:52:05 


\title{
АППРОКСИМАЦИИ ПАДЕ И ЭФФЕКТИВНОЕ АНАЛИТИЧЕСКОЕ ПРОДОЛЖЕНИЕ СТЕПЕННОГО РЯДА
}

\author{
С. П. СуЕтин
}

Обзор отражает современное состояние теории аппроксимаций Паде - наилучших рациональных приближений степенного ряда. Главное внимание уделяется так называемьм обратным задачам этой теории, в которых требуется сделать те или иные вьводы об аналитическом продолжении заданного степенного ряда на основании известного асимптотического поведения полюсов некоторой последовательности его аппроксимаций Паде. С этой точки зрения в работе рассматриваются строчные и диагональные последовательности. Кроме результатов автора, приводятся фундаментальные резултаты обратного характера, принадлежащие А.А. Гончару и Е.А. Рахманову.

Библиография: 79 названий.

\section{СОДЕРЖАНИЕ}

Введение

Глава I. Полюсы строк таблицы Паде и особые точки аналитической функции

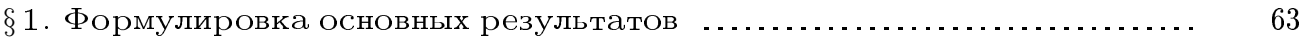

$\S 2$. Доказательство теоремы 1 ................................... 65

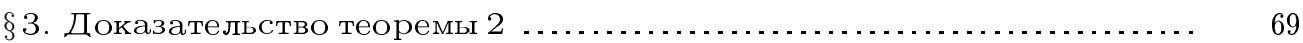

Глава II. Равномерная сходимость диагональных аппроксимаций Паде для некоторых классов аналитических функций $\ldots \ldots \ldots \ldots \ldots \ldots .63$

$\S 1$. Постановка задачи и формулировка основных результатов ........ 83

$\S 2$. Основные определения и вспомогательные результаты . ........... 95

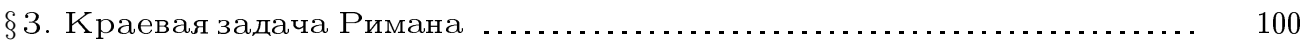

$\S 4$. Построение "явного" решения проблемы обрашения Якоби . ........ 108

$\S 5$. Доказательство основных результатов ......................... 111

Глава III. Аппроксимации Паде ортогональных разложений ........... 116

$\S 1$. Основные понятия и результаты $\ldots . \ldots \ldots \ldots \ldots \ldots \ldots \ldots \ldots \ldots \ldots \ldots \ldots . . \ldots \ldots$

$\S 2$. Доказательство предложения 3.1 ............................... 121

$\S 3$. Доказательство теоремы 6 ................................... 128

Список литературы ............................................... 139

Работа выполнена при поддержке Российского фонда фундаментальных исследований (грант № 99-01-01149) и фонда INTAS (грант № 2000-272). 


\section{Введение}

1. Задача эффективного аналитического продолжения заданной степенньм рядом аналитической функции и локализации ее особенностей непосредственно по коэффициентам этого ряда является классической задачей комплексного анализа. Как известно, указанная проблема возникла под влиянием вейерштрассовского подхода к понятию аналитической функции, которьй исходит из того, что функция задается аналитическим элементом - сходяшимся степенным рядом

$$
f(z)=\sum_{k=0}^{\infty} c_{k} z^{k}
$$

и ставит своей цель выразить свойства аналитической функции непосредственно в терминах коэффициентов этого ряда. Фундаментальные результаты в этом направлении были получены еще в конще XIX века. Наиболее известными из них являются теорема Адамара о нахождении радиусов кругов мероморфности аналитической функции по коэффициентам ее разложения в степенной ряд и теорема Фабри "об отношении”. К этому же кругу вопросов относятся исследования Чебьшёва, Маркова и Стилтьеса о непрерьвных дробях, которые также строятся непосредственно по коэффициентам степенного ряда. Эти и другие результаты, как оказалось впоследствии, допускают естественную интерпретацию в терминах конструктивных рациональных приближений степенного ряда - аппроксимаций Паде. В рамках исследования сходимости таких аппроксимаций удалось разработать достаточно общий подход к проблеме эффективного аналитического продолжения функций и получить естественное обобщение некоторых классических задач.

Как известно, аппроксимации Паде - это локально наилуишие рациональные аппроксимации заданного степенного ряда. Они конструируются непосредственно по его коэффициентам и позволяют осушествлять эффективное аналитическое продолжение этого ряда за пределы его круга сходимости, а их полюсы в определенном смысле локализуют особые точки (в том числе полюсы и их кратности) продолженной функции в соответствующей области сходимости и на ее границе. Последнее свойство аппроксимаций Паде основано на том, что все их полюсь “свободнь” " и определяются только условием максимальности касания заданного степенного ряда. Этим апшроксимации Паде принципиально отличаются от рациональных аппроксимаций с (полностью или частично) фиксированными полюсами, в том числе от полиномиальных приближений, в случае которых все полюсы фиксированы в одной, бесконечно удаленной, точке.

Именно указанное вьше свойство аппроксимаций Паде - эффективно решать задачу аналитического продолжения степенного ряда - и лежит в основе их многочисленных успешных применений в анализе и при исследовании прикладных задач. В настояшее время метод аппроксимации Паде является одним из наиболее перспективных нелинейных методов суммирования степенного ряда и локализации его особых точек. В том числе и по этой причине теория аппроксимаций Паде превратилась во вполне самостоятельньй раздел теории приближений, а сами эти аппроксимации нашли разнообразные применения как непосредственно в теории рациональных приближений, так и в теории чисел, теории несамосопряженных операторов, исследовании дифференщиальных уравнений, зависящих от малого параметра, в теории возмущений (см. [1]-[12], а также монографиию [13], где имеются дальнейшие ссылки). 
Для заданного степенного ряда $(*)$ и произвольной пары неотрицательных целых чисел $n, m$ аппрожсимачией Паде $[n / m]_{f}$ типа $(n, m)$ ряда $f$ называется (единственная) рациональная функция, доставляющая максимально возможный порядок касания к этому ряду в начале координат в классе функций $\mathscr{R}_{n, m}=\left\{r \in \mathbb{C}(z): r=\left(a_{0}+\right.\right.$ $\left.\left.a_{1} z+\cdots+a_{n} z^{n}\right) /\left(b_{0}+b_{1} z+\cdots+b_{m} z^{m}\right)\right\}$. Соответствуюшая таблица $\left\{[n / m]_{f}\right\}_{n, m=0}^{\infty}$ называется таблицей Паде ряда $f$, последовательности вида $\left\{[n / m]_{f}\right\}, n=0,1,2, \ldots$, где $m \in \mathbb{Z}_{+}-$фиксировано, назьваются строчными последовательностями (или "строками" ) в таблище Паде, а последовательность $\left\{[n / n]_{f}\right\}, n=0,1,2, \ldots,-$ диагональной последовательностью (или “главной диагональю”).

Вопрос о мероморфном восстановлении функции $f$ по степенному ряду $(*)$ в ее так называемом максимальном круге $m$-мероморфности $D_{m}(f)$ (где $f$ мероморфна и имеет $\leqslant m$ полюсов) при условии, что $f$ имеет в $D_{m}(f)$ в точности $m$ полюсов (как обычно, полюсы функций считаются с учетом их кратностей), решается классической теоремой Монтессу де Болора [14].

Теорема Монтессу ДЕ БолОРА. Пусть функция $f$ имеет ровно $m$ полюсов в круге $D_{m}(f):|z|<R$. Тогда:

$1^{\circ}$. При всех достаточно больших $n$ аппроксимации Паде $[n / m]_{f}$ ряда $f$ имеют ровно $m$ конечных полюсов, которые при $n \rightarrow \infty$ стремятся $\kappa$ полюсам функции $f$ в $D_{m}(f)$, причем каждый полюс $f$ “притягивает" столько полюсов $[n / m]_{f}$, какова его кратность.

$2^{\circ}$. Последовательность $[n / m]_{f}, n=0,1,2, \ldots$, сходится $к$ функиии $f$ равномерно внутри (т.е. на компактных подмножествах) области $D_{m}^{\prime}$, которая получается из $D_{m}$ удалением полюсов функции $f$.

При этом в условиях теоремы скорость сходимости последовательности $[n / m]_{f}$ к функции $f$ в $D_{m}^{\prime}$ характеризуется неравенством:

$$
\varlimsup_{n \rightarrow \infty}\left|f(z)-[n / m]_{f}(z)\right|^{1 / n} \leqslant \frac{|z|}{R}<1 .
$$

При доказательстве своего результата Монтессу де Болор в существенной степени опирался на полученные ранее Адамаром [15] непосредственно в терминах коэффициентов ряда $(*)$ формулы для радиусов $R=R_{m}(f)$ кругов $D_{m}(f)$. Точнее, пусть

$$
\left.H_{n, m}=\left|\begin{array}{cccc}
c_{n-m+1} & c_{n-m+2} & \ldots & c_{n} \\
\ldots \ldots \ldots \ldots \ldots \ldots \ldots \ldots & \ldots \ldots \ldots \ldots \\
c_{n} & c_{n+1} & \ldots & c_{n+m-1}
\end{array}\right| \text { (полагаем } c_{k}=0 \text { при } k<0\right) .
$$

Справедлива следуюшая

Теорема АДАмАРА. Для произвольного $m \in \mathbb{N} \in \mathbb{Z}_{+}$

$$
R_{m}=\frac{\ell_{m}}{\ell_{m+1}}, \quad \text { zдe } \quad \ell_{j}=\varlimsup_{n \rightarrow \infty}\left|H_{n, j}\right|^{1 / n}
$$

$\left(\ell_{0}=1 ;\right.$ ecлu $\ell_{1}, \ldots, \ell_{m} \neq 0, \ell_{m+1}=0$, mo $\left.R_{m}=\infty\right)$.

Из теоремы Монтессу де Болора уже легко следует, что конечные полюсы рациональных функций $[n / m]_{f}$ стремятся к полюсам $f$ со скоростью геометрической прогрессии. 
На самом деле это свойство полюсов функций $[n / m]_{f}$ является характеристическим. Это вытекает непосредственно из полного описания $m$-мероморфного продолжения степенного ряда $f$ с помощью $m$-й строки таблицы Паде при произвольном $m \in \mathbb{N}$, полученного А.А. Гончаром [16].

В терминах, связанных с асимптотическим поведением конечных полюсов $m$-й строки таблицы Паде, в [16] получены формулы для радиуса $m$-го круга мероморфности и дивизора полюсов продолженной функции $f$ внутри этого круга, а также доказана общая теорема о сходимости $m$-й строки таблищы Паде по (логарифмической) емкости внутри $D_{m}(f)$ : результат Монтессу вытекает из нее как частный случай.

Естественным образом возникает следующий более общий вопрос: какие выводы можно сделать о функции $f$ в целом, если известно, что конечные полюсы $m$-й строки таблицы Паде стремятся к некоторым точкам в комплексной плоскости без какого-либо предположения о скорости этой сходимости. Рассмотрим первую строку, т.е. случай $m=1$. Если $c_{n} \cdot c_{n+1} \neq 0$, то единственный конечньй полюс $\zeta_{n}$ рациональной функции $[n / 1]_{f}$ вычисляется по формуле $\zeta_{n}=c_{n} / c_{n+1}$. Таким образом, соотношение $\zeta_{n} \rightarrow a \in \mathbb{C}^{*}=\mathbb{C} \backslash\{0\}$ эквивалентно тому, что $c_{n} / c_{n+1} \rightarrow a$ при $n \rightarrow \infty$, и мы оказьваемся в условиях классической теоремы Фабри "об отношении" [17] (см. также монографию [18]).

ТЕОРЕМА ФАБРИ. Если для коэффициентов степенного ряда (*) имеет место соотношение

$$
\lim _{n \rightarrow \infty} \frac{c_{n}}{c_{n+1}}=a
$$

то $z=а$ - особая точка суммы этого ряда на границе его круга сходимости $|z|<R_{0}, R_{0}=|a|$.

Тем самым, для $m=1$ теорема Фабри фактически устанавливает связь между асимптотическим поведением конечного полюса первой строки таблицы Паде и особьми точками функции $f$ на гранище круга голоморфности $D_{0}(f)$. Обсуждение аналогичной проблемы для произвольного $m \in \mathbb{N}$ является одной из основных целей настоящего обзора.

Из сказанного вьше вытекает, что строчные последовательности аппроксимаций Паде приспособленыпрежде всего для описания мероморфных продолжений ряда (*) в соответствуюшие круги. Присутствие на гранище круга сходимости этого ряда какой-либо особенности иного характера, например точки ветвления, приводит к тому, что никакая строчная последовательность не эффективна при решении задачи аналитического продолжения.

Иначе обстоит дело с диагональными последовательностями аппроксимаций Паде. Одним из первых результатов общего характера о сходимости таких рациональных аппроксимаций аналитических функций является классическая теорема Маркова [19], полученная им в терминах чебьшёвских непрерьвных дробей для функций вида

$$
\widehat{\mu}(z):=\int_{S} \frac{d \mu(\zeta)}{z-\zeta}
$$

где $\mu$-положительная борелевская мера с носителем $S=S_{\mu} \Subset \mathbb{R}$. 
Теорема Маркова. Для функиии $\widehat{\mu}$ вида $(* *)$ с носителем $S_{\mu} \Subset \mathbb{R}$, cocтоящим из бесконечного множества точек, диагональные аппроксимации Паде $[n / n]_{\widehat{\mu}}$, построенные по коэффициентам разложения $\widehat{\mu}$ в ряд Лорана в точке $z=\infty$, сходятся $\kappa \widehat{\mu}$ равномерно внутри области $\overline{\mathbb{C}} \backslash[a, b]$, әде $[a, b]-$ минимальный отрезок вещественной оси, содержащий $S_{\mu}$.

Тем самым, любая марковская функиия (т.е. функция вида $(* *)$ с $\left.S_{\mu} \Subset \mathbb{R}\right)$ может быть восстановлена вне выпуклой оболочки $\widehat{S_{\mu}}=[a, b]$ носителя меры по коэффициентам своего лорановского разложения в точке $z=\infty$ (моментам меры $\mu$ ).

Тот факт, что в теореме Маркова речь идет о равномерной сходимости главной диагонали Паде не в $\overline{\mathbb{C}} \backslash S_{\mu}$ - области голоморфности функции $\widehat{\mu}$, а лишь вне выпуклой оболочки $\widehat{S_{\mu}}$ носителя меры, связан с существом дела: в наиболее типичной ситуации множество предельных точек полюсов рациональных функций $[n / n]_{\widehat{\mu}}$ совпадает с $\widehat{S_{\mu}}$. В обшем же случае, когда носитель меры $\mu$ в $(* *)$ не лежит на прямой, предельные точки полюсов диагональных аппроксимаций Паде могут составлять аналитические дуги в области $D=\overline{\mathbb{C}} \backslash S_{\mu}$ и даже быть всюду плотными в $\overline{\mathbb{C}}$. Более точно, по некоторым подпоследовательностям полюсы аппроксимаций Паде могут сходиться к любой наперед заданной точке аналитической дуги или, соответственно, к произвольной точке $\overline{\mathbb{C}}$. В такой ситуации принщипиальным становится вопрос о том, может ли какой-либо полюс апроксимаций Паде иметь предел (а не только предельную точку) по всей последовательности $n \in \mathbb{N}$, отличный от полюса функции $f$. Этот вопрос непосредственно связан с задачей о восстановлении диагональю Паде дивизора полюсов мероморфной в $\overline{\mathbb{C}} \backslash S_{\mu}$ функции вида

$$
f=\widehat{\mu}+r,
$$

где $r \in \mathbb{C}(z)$ - рациональная функция, голоморфная на $[a, b]$ ( $f$ - "рациональное возмущение" марковской функции $\widehat{\mu})$. Конструкция аппроксимаций Паде существенно нелинейна, поэтому исследование сходимости таких аппроксимаций для функций вида $(* * *)$ - сложная задача. Положительное решение задачи о восстановлении дивизора вытекает непосредственно из сушествования подпоследовательности главной диагонали, равномерно сходящейся в сферической метрике (расстояние в которой измеряется длиной меньшей дуги между соответствуюшими точками на сфере Римана) внутри $\overline{\mathbb{C}} \backslash S_{\mu}$ к мероморфной функции $f$. Обзор полученных результатов по этой проблеме для класса функций вида (***) является одной из основных целей статьи.

В последнее время [13] в связи с некоторьми задачами математической физики естественным образом возник интерес к изучению аппроксимаций Паде общих ортогональных разложений, прежде всего - разложений по полиномам Лежандра и Чебьшёва, приводяшим к представлению “заданной” функции в виде ряда Фурье по ортогональным многочленам. При этом, так же как и в классическом случае, особый интерес представляет вычисление значений функции вне пределов "канонической" области сходимости ортогонального разложения, а коэффициенты разложения определяются по таким рекуррентным формулам, что с ростом номера коэффициента резко возрастает объем вычислений, а следовательно, и "стоимость" численного нахождения коэффициентов. Метод аппроксимаций Паде ортогональных разложений является хорошо проявившим себя (нелинейным) методом обработки найденных коэффиициентов Фурье и эффективного аналитического продолжения соответствующего ряда за 
пределы его канонической области сходимости. Обсуждение вопросов сходимости таких рациональных аппроксимаций составляет содержание последней, третьей, главы работы.

Наконец, прежде чем переходить к изложению основного содержания обзора, упомянем о работах Н.У. Аракеляна и его учеников [20]-[23], в которых получили дальнейшее развитие другие классические результаты теории аналитического продолжения (см. прежде всего монографию [18]), относяшиеся к задаче локализации особых точек степенного ряда $(*)$ на границе круга сходимости, а также находящихся в вершинах главной звезды Миттаг-Леффлера, в том числе и за пределами этого круга. Кроме того, в [20]-[23] исследуется и задача эффективного аналитического продолжения степенного ряда за границу его круга сходимости, точнее - задача продолжения в главную звезду Миттаг-Леффлера и в произвольную спиральную звезду. В качестве способа продолжения предлагается линейный метод суммирования с помощю некоторой бесконечной матрицы, элементы которой зависят от параметра. Частными случаями такого способа являются классические методы суммирования Миттаг-Лефффлера и Линделёфа.

2. Статья состоит из введения и трех глав.

В первой главе рассматривается связь между асимптотическим поведением полю-

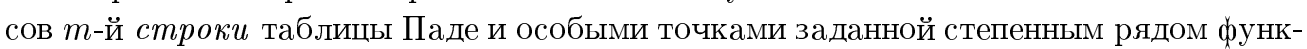
ции как внутри, так и на границе $m$-го круга мероморфности. Главньм результатом этой главы является обобщение классической теоремы Фабри “об отношении” на случай произвольной строки таблицы Паде степенного ряда. В основе доказательства обобщенного варианта теоремы Фабри лежит модификация метода Агмона - одного из классических методов, основанного на связи между опорной функцией и индикатором для соответственно нижней и верхней функций, ассоциированных по Борелю. Основные результаты этой главы опубликованы в работах автора [24], [25].

Пусть

$$
f=\sum_{k=0}^{\infty} c_{k} z^{k}
$$

- произвольный степенной ряд, $R_{0}=R_{0}(f)>0$ - радиус его сходимости. Для любых неотрицательных целых чисел $n, m$ сушествует пара полиномов $p, q \in \mathbb{C}[z]$ таких, что $\operatorname{deg} p \leqslant n, \operatorname{deg} q \leqslant m, q \not \equiv 0$ и выполняется следуюшее соотношение:

$$
(q f-p)(z)=O\left(z^{n+m+1}\right), \quad z \rightarrow 0
$$

Полиномы $p, q$, удовлетворяюшие системе (0.2), определяются, вообще говоря, не единственньм образом, однако их отношение $p / q$ задает единственную рациональную функцию - аппроксимацию Паде $[n / m]_{f}$ типа $(n, m)$ ряда $(0.1)$, доставляющую максимально возможньй порядок касания к этому ряду в классе $\mathscr{R}_{n, m}=\{r \in \mathbb{C}(z)$ : $\left.r=\left(a_{0}+a_{1} z+\cdots+a_{n} z^{n}\right) /\left(b_{0}+b_{1} z+\cdots+b_{m} z^{m}\right)\right\}$ в начале координат.

Зафиксируем произвольное натуральное число $m$ и рассмотрим $m$-ю строку таблищы Паде ряда $(0.1):[n / m]_{f}, n=0,1, \ldots$

Пусть $\mathscr{P}_{n}=\left\{\zeta_{n, 1}, \ldots, \zeta_{n, m_{n}}\right\}, 0 \leqslant m_{n} \leqslant m,-$ множество конечных полюсов рациональной функции $[n / m]_{f}$. Введем следуюшие характеристики асимптотического 
поведения последовательности множеств $\mathscr{P}_{n}, n=0,1, \ldots$, в окрестности произвольной точки $a \in \mathbb{C}^{*}=\mathbb{C} \backslash\{0\}: \mu(a)$ - число точек множества $\mathscr{P}_{n}$, стремяшихся к точке $a$ со скоростью геометрической прогрессии при $n \rightarrow \infty ; \mathscr{P}=\left\{a \in \mathbb{C}^{*}: \mu(a) \geqslant 1\right\}$;

$$
\Delta(a)=\varlimsup_{n \rightarrow \infty} \prod_{j=1}^{m_{n}}\left|a ; \zeta_{n, j}\right|^{1 / n},
$$

где $|\cdot ; \cdot|$ - расстояние между точками в сферической метрике.

Очевидно, $\mu(a) \geqslant 1 \Longleftrightarrow \Delta(a)<1$.

Через $D_{m}=D_{m}(f):|z|<R_{m}$ обозначим максимальньй открытьй круг с центром в точке $z=0$, в которьй функция $f$ продолжается как мероморфная функция, имеюшая $\leqslant m$ полюсов $\left(R_{m}=R_{m}(f)\right.$ - радиус $m$-мероморфности функции $\left.f\right)$.

А. А. Гончар [16] в терминах характеристик $\mu$ и $\Delta$ полностью описал $m$-меромор ффное продолжение ряда $(0.1)$.

ТеОРема ГОНЧАРА. Пусть $f-p_{я} \partial(0.1), m \in \mathbb{N}$ и $a \neq 0-$ фиксированная точка комплексной плоскости. Следующие утверждения әквивалентны:

$1^{\circ}$. $R_{m}>|a|$ u $f$ имеет полюс в точке $а$;

$2^{\circ} . \Delta(a)<1$ (или, что то же самое, $\left.\mu(a) \geqslant 1\right)$.

При әтом (если выполнено какое-либо из условий $1^{\circ}$ и $2^{\circ}$ ) кратность полюса $f \boldsymbol{\theta}$ точке а равна $\mu($ а) и справедлива формула

$$
R_{m}=\frac{|a|}{\Delta(a)} .
$$

Тем самым, если $d_{m}=\left\{\left(a_{1}, \nu_{1}\right), \ldots,\left(a_{s}, \nu_{s}\right)\right\}$ - дивизор полюсов функции $f$ в круге $D_{m}\left(a_{j}\right.$ - полюсы $f$ в $D_{m}, \nu_{j} \geqslant 1$ - кратность полюса в точке $a_{j}$; $\left|d_{m}\right|=\nu_{1}+\cdots+\nu_{s} \leqslant m-$ число полюсов $f$ в $\left.D_{m}\right)$, то из теоремы Гончара вытекает формула для вычисления дивизора полюсов функции $f$ в круге $D_{m}$ при любом $m \in \mathbb{N}$ : дивизор $d_{m}=\{(a, \mu(a)): a \in \mathscr{P}\}$. Утверждение классической теоремы Монтессу де Болора по существу состоит в том, что эта формула для вычисления дивизора функции $f$ справедлива при условии, что $\left|d_{m}\right|=m$.

Естественным образом возникает следующий вопрос: какие вьводы можно сделать о функции $f$ в целом, если известно, что конечные полюсы $m$-й строки таблищы Паде стремятся к некоторым точкам в комплексной плоскости без какого-либо предположения о скорости этой сходимости.

Основньми результатами первой главы являются следующие теоремы.

TeOpema 1. Пусть $\operatorname{dist}\left(a, \mathscr{P}_{n}\right) \rightarrow 0$ nри $n \rightarrow \infty$ для некоторой точки $a \neq 0$ плоскости $\mathbb{C}$. Тогда $R_{m} \geqslant|a|$. Если при этом $|a|<R_{m}$, то а- полюс функиии $f$.

ТЕОрема 2. Пусть для всех достаточно больших $n$ рациональные функции $[n / m]_{f}$ имеют ровно $m$ конечных полюсов $\zeta_{n, 1}, \zeta_{n, 2}, \ldots, \zeta_{n, m}$, причем

$$
\zeta_{n, j} \rightarrow a_{j} \in \mathbb{C}^{*}, \quad n \rightarrow \infty, \quad j=1,2, \ldots, m
$$

əde

$$
0<\left|a_{1}\right| \leqslant \cdots \leqslant\left|a_{\mu-1}\right|<\left|a_{\mu}\right|=\cdots=\left|a_{m}\right|=R .
$$


Tогда:

$1^{\circ} . R_{\mu-1}(f)=\cdots=R_{m-1}(f)=R$ и все точки $a_{1}, \ldots, a_{\mu-1}$ и только они полюсы функции $f$ в круге $|z|<R$.

$2^{\circ}$. Все точки $a_{\mu}, \ldots, a_{m}$ - особье точки функиии $f$ на границе круга $|z|<R$.

Теорема 2 обобщает теорему Фабри “об отношении” на случай $m$-й строки таблищы Паде.

Некоторые другие вопросы сходимости строк таблицы Паде степенного ряда изучены в работах [26], [27].

3. Во второй главе рассматривается задача о восстановлении главной диагональю Паде дивизора полюсов находящейся в “общем положении” произвольной функции из класса Наттолла, в частности доказьвается один из вариантов гипотезы Бейкера-Гамелля-Уиллса о существовании равномерно сходящейся в сферической метрике подпоследовательности диагональных аппроксимаций Паде. Анализ равномерной сходимости главной диагонали Паде в настояшей работе опирается на хорошо известньй факт, что для функций класса Наттолла знаменатели таких аппроксимаций суть полиномы, неэрмитово ортогональные относительного комплексного веса на специальных аналитических дугах, обладающих определенным свойством "симметрии". Сильная асимптотика таких ортогональных полиномов описывается в терминах решений определенной краевой задачи Римана на соответствующей гиперэллиптической римановой поверхности. В свою очередь, анализ существования и нахождение "явного" вида решения такой задачи дается в работе в терминах обладающего спешиальными свойствами решения проблемы Якоби обрашения абелевых интегралов. Основные представленные здесь результаты второй главы опубликованы в работах автора [28], [29].

Пусть

$$
f(z)=\sum_{k=0}^{\infty} \frac{c_{k}}{z^{k+1}}
$$

- функция, голоморфная в бесконечно удаленной точке $z=\infty$. Если все ганкелевы определители, составленные из коэффициентов $\left\{c_{k}\right\}$, отличны от нуля, то $f$ можно сопоставить чебьшёвскую непрерьвную дробь

$$
\frac{b_{1}}{z-a_{1}-\frac{b_{2}}{z-a_{2}-\ddots}}
$$

подходящие дроби $P_{n} / Q_{n}$ которой обладают следуюшим замечательным свойством:

$$
\frac{P_{n}}{Q_{n}}(z)=\sum_{k=0}^{2 n-1} \frac{c_{k}}{z^{k+1}}+\frac{A_{n}}{z^{2 n+1}}+\cdots,
$$

тем самьм,

$$
\left(f-\frac{P_{n}}{Q_{n}}\right)(z)=O\left(\frac{1}{z^{2 n+1}}\right) .
$$


Последнее соотношение единственным образом определяет рациональную функцию $P_{n} / Q_{n}$ в классе всех рациональных дробей порядка не вьше $n$. Такая дробь доставляет наилучшее локальное приближение ряда (0.3) в указанном классе функций. Это свойство служит определением диагональньх аппроксимаций Паде $[n / n]_{f}=P_{n} / Q_{n}$ для произвольного (формального или сходяшегося) ряда (0.3).

П. Л. Чебьшёв [30] впервые рассмотрел формальные разложения в непрерьвные дроби для функций вида

$$
\widehat{\mu}(z):=\int_{S} \frac{d \mu(\zeta)}{z-\zeta}
$$

где $\mu$ - положительная мера с носителем $S=S_{\mu} \subset \mathbb{R}$. Именно на этом пути Чебышёв открыл и исследовал обшие ортогональные многочлены, соответствующие произвольной мере на $\mathbb{R}$ : такие многочлены естественным образом возникают как знаменатели $Q_{n}=Q_{n}(z ; \widehat{\mu})$ подходяших дробей функции $\widehat{\mu}$ вида $(0.4)$.

Одним из первых результатов обшего характера о сходимости диагональных аппроксимаций Паде аналитических функций является классическая теорема Маркова [19], полученная им в терминах чебышёвских непрерывных дробей, из которой вытекает, что любая марковская функиия (т.е. функция вида $(0.4)$ с носителем $\left.S_{\mu} \Subset \mathbb{R}\right)$ может быть восстановлена вне вьпуклой оболочки $\widehat{S_{\mu}}=[a, b]$ носителя меры по коэффициентам своего лорановского разложения в точке $z=\infty$ (моментам меры $\mu$ ).

Отметим, что установленное в этой теореме свойство диагональных аппроксимаций Паде относится и к следуюшим әлементарным функииям:

$$
\widehat{\mu}(z)=\frac{1}{\sqrt{z^{2}-1}} \text { и } \widehat{\mu}(z)=\log \frac{z+1}{z-1}
$$

где в первом случае $d \mu(x)=d x /\left(\pi \sqrt{1-x^{2}}\right)$, а во втором $d \mu(x)=d x-$ положительные меры на $S_{\mu}=[-1,1]$; соответственно этому знаменатели рациональных функций $[n / n]_{\widehat{\mu}}$ - это полиномы Чебьшёва (первого рода) и полиномы Лежандра. В обоих случаях $\widehat{\mu}(z)$ - голоморфная функция в области $\overline{\mathbb{C}} \backslash[-1,1]$, ряд Лорана которой сходится лишь во внешности единичного круга $\{z \in \overline{\mathbb{C}}:|z|>1\}$.

$\mathrm{K}$ теореме Маркова непосредственно примыкают результаты А. А. Гончара [31] и Е. А. Рахманова [32] о сходимости диагональных аппроксимаций Паде для мероморфньъ $x$ функций $f$ вида

$$
f=\widehat{\mu}+r
$$

где $\widehat{\mu}$ - марковская функция, $r$ - рациональная функция, голоморфная на $[a, b]=\widehat{S_{\mu}}$ $(r \in \mathbb{C}(z) \cap \mathscr{H}[a, b], f$ - "рациональное возмушение" $\widehat{\mu})$. Конструкция аппроксимаций Паде существенно нелинейна, поэтому исследование сходимости таких рациональных приближений для функций вида (0.5) - нетривиальная задача. В теореме Гончара утверждается, что диагональнье аппроксимачии Паде $[n / n]_{f}$ сходятся равномерно в сферической метрике $к$ функиии $f$ внутри области $\overline{\mathbb{C}} \backslash[a, b]$ при условии, что $S_{\mu}=[a, b]$ и $\mu^{\prime}(x)=d \mu / d x>0$ почти всюдун на $[a, b]$, а функция $r \in \mathbb{C}(z)$ $u$ голоморфна на $[a, b]$. В работе Рахманова аналогичньй результат о равномерной сходимости $[n / n]_{f}$ в сфферической метрике вне $[a, b]$ установлен в предположении, что $\widehat{\mu}-$ произвольная марковская функиия, а функиия $r \in \mathbb{R}(z)$ и голоморфна на $[a, b]$. 
Тем самым, диагональные аппроксимации Паде в классе функций вида (0.5) при определенных условиях эффективно решают задачу мероморфного продолжения в область $\overline{\mathbb{C}} \backslash[a, b]$ функции, заданной своим разложением в ряд Лорана в бесконечно удаленной точке: полюсы этих аппроксимаций локализуют отрезок $[a, b]$ и особые точки (полюсы и их кратности) функции $f$ в $\overline{\mathbb{C}} \backslash[a, b]$, а сами аппроксимации осуществляют мероморфное продолжение ряда $f$ в $\overline{\mathbb{C}} \backslash[a, b]$.

Тот факт, что в теореме Маркова речь идет о равномерной сходимости не в $\overline{\mathbb{C}} \backslash S_{\mu}-$ области голоморфности функции $\widehat{\mu}$, а лишш вне вьпуклой оболочки $\widehat{S_{\mu}}$ носителя меры, связан с сушеством дела: непосредственно из открытого Чебьшёвым свойства ортогональности знаменателей диагональных аппроксимаций Паде функции вида (0.4) вытекает, что если носитель меры $\mu$ состоит из нескольких непересекаюшихся отрезков, то в лакунах между этими отрезками могут располагаться полюсы рациональной функции $[n / n]_{\widehat{\mu}}$. Более того, как показано автором (подробнее см. главу II), "в общем положении" множество предельных точек полюсов рациональных функций $[n / n]_{\widehat{\mu}}$ - нулей соответствуюших ортогональных полиномов - совпадает $c \widehat{S_{\mu}}$, что делает принципиально невозможной равномерную сходимость $[n / n]_{\widehat{\mu}}$ внутри $\overline{\mathbb{C}} \backslash S_{\mu}$ в классе всех марковских функций (0.4). Аналогичная ситуация, когда полюсы диагональных аппроксимаций Паде имеют предельные точки в области мероморфности заданной функции вида (0.5), не соответствуюшие полюсам рациональной функции $r$, имеет место и в условиях теоремы Рахманова. Если же в этом случае допустить, что $r \in \mathbb{C}(z)$, а $S_{\mu}$ состоит в точности из двух отрезков, то, как показано в [32], существует аналитическая дуга $\gamma$, соединяющая эти отрезки и лежашая в $\overline{\mathbb{C}} \backslash[a, b]$, такая, что равномерная сходимость апроксимаций Паде $[n / n]_{f}$ к функции $f$ в сферической метрике имеет место в $\overline{\mathbb{C}} \backslash\left(S_{\mu} \cup \gamma\right)$. При этом если число $\omega_{1}(\infty)$ - гармоническая мера первого отрезка относительно области $\overline{\mathbb{C}} \backslash S_{\mu}$ в бесконечно удаленной точке - иррационально, то сходимость не имеет места ни в какой области, пересекающейся с $\gamma$; в противном случае сушествует конечное множество точек $\mathscr{P} \subset \gamma$ такое, что равномерная сходимость имеет место в $\overline{\mathbb{C}} \backslash\left(S_{\mu} \cup \mathscr{P}\right)$. Дуга $\gamma$ полностью определяется "геометрией” задачи: она зависит только от $S_{\mu}$ и расположения полюсов функции $r$ и не зависит от меры $\mu$ и вычетов функции $r$ в полюсах.

В общем случае, когда носитель меры $\mu$ в (0.4) не лежит на прямой, предельные точки полюсов диагональных аппроксимаций Паде могут составлять аналитические дуги в области $D=\overline{\mathbb{C}} \backslash S_{\mu}$ и даже быть всюду плотными в $\overline{\mathbb{C}}$. Более точно, по некоторым подпоследовательностям полюсы аппроксимаций Паде могут сходиться к любой наперед заданной точке аналитической дуги или, соответственно, к произвольной точке $\overline{\mathbb{C}}$. В такой ситуации принципиальным становится вопрос о том, может ли какой-либо полюс аппроксимаций Паде иметь предел (а не только предельную точку) по всей последовательности $n \in \mathbb{N}$, отличный от полюса функции $f:$ этот вопрос связан с важной задачей о восстановлении диагональю Паде дивизора полюсов мероморфной в $\overline{\mathbb{C}} \backslash S_{\mu}$ функции $f$.

Основная цель второй главы работы - доказательство некоторых результатов достаточно общего характера о восстановлении главной диагональю Паде дивизора полюсов мероморфной функции. Методы, используемые нами во второй главе, таковы, что эти результаты вытекают непосредственно из положительного решения задачи о сушествовании для произвольной функции из достаточно широкого класса некоторой 
подпоследовательности диагональных аппроксимаций Паде, равномерно сходяшейся в сферической метрике к этой функции в ее области мероморфности. Как известно, вопрос о существовании такой подпоследовательности в более общей ситуации составляет содержание широко известной гипотезы Бейкера-Гамелля-Уиллса (подробнее см. [33]). Недавно В.И. Буслаевым ${ }^{1}$ построен пример гиперэллиптической функции, опровергающий эту гипотезу как в первоначальной постановке, так и в более широком смысле, предложенном в [33]. Отметим, что в случае Буслаева не вьполняются условия "обшего положения", предполагаемые в этом обзоре.

Наиболее обший результат о сходимости диагональных аппроксимаций Паде был получен Шталем [34]. Для некоторого естественного класса функций вида (0.5), содержащего все алгебраические функции, Шталь доказал сходимость диагональных последовательностей аппроксимаций Паде по емкости. Сходимость диагональных аппроксимаций Паде непосредственно связана с асимптотикой их знаменателей многочленов, неэрмитово ортогональных на дугах, составляющих $S$, относительно меры $\mu$. Подход Шталя фактически основан на изучении так назьваемой "слабой" асимптотики таких многочленов.

Во второй главе настоящей работы мы развиваем подход Наттолла, в основе которого - изучение “сильной” (т.е. типа Бернштейна-Сегё) асимптотики знаменателей аппроксимаций Паде. В рамках такого подхода Наттолл [9], [35] получил результат о сходимости по емкости последовательности диагональных апшроксимаций Паде функции класса (0.5) при условии, что носитель $S$ меры $\mu$ - компакт "минимальной емкости”, соответствующий некоторой многозначной функции вида $\sqrt{\left(z-e_{1}\right) \cdots\left(z-e_{2 \mathrm{~g}+2}\right)}$, где точки $e_{1}, \ldots, e_{2 \mathrm{~g}+2} \in \mathbb{C}$ попарно различны, $\mathrm{g} \geqslant 0$. Сама же мера в этом случае предполагается достаточно гладким комплекснозначньм весом на $S$. Этот класс функций содержит обшие гиперәллиптические функции.

Отметим, что случай эллиптической функции $f \in \mathscr{H}(\infty)$ вида

$$
f(z)=\sqrt{\left(z-e_{1}\right) \cdots\left(z-e_{4}\right)}-z^{2}+z\left(e_{1}+\cdots+e_{4}\right) / 2
$$

где точки $e_{1}, \ldots, e_{4} \in \mathbb{C}$ попарно различны и выбрана та ветвь корня, старший член которой в окрестности бесконечно удаленной точки равен $z^{2}$, был исследован Дюма [36] в терминах непрерьвных дробей. Он показал, что если некоторые числовые параметры, связанные только с взаимньм расположением точек $e_{1}, \ldots, e_{4}$, рационально независимы, то множество полюсов диагональных аппроксимаций Паде всюду плотно в $\overline{\mathbb{C}}$, т.е. последовательность $[n / n]_{f}$ не может равномерно сходиться ни в какой подобласти $\mathbb{C}$. Однако эта последовательность в силу теоремы Наттолла сходится по емкости в дополнении к разрезам минимальной емкости.

В настояшей работе предполагается, что мера $\mu$ такова, что функщия $\widehat{\mu}$ представляется в виде

$$
\widehat{\mu}(z)=\frac{1}{2 \pi i} \int_{S} \frac{\rho(\zeta)}{z-\zeta} \frac{d \zeta}{\sqrt{h(\zeta)}}=: \widehat{\rho}(z ; h),
$$

где $h=h_{2 \mathrm{~g}+2} \in \mathbb{C}[z]-$ некоторьй полином четной степени $2 \mathrm{~g}+2, \mathrm{~g} \geqslant 0$, с единичным старшим коэффициентом, все корни $\left\{e_{j}\right\}$ которого - простые, $S=S(h)-$

\footnotetext{
${ }^{1}$ V.I. Buslaev, Simple counterexample to the Baker-Gammel-Wills conjecture, "East Journal on Approximations", 2001, № 4, p. 515-517.
} 
компакт, имеющий минимальную емкость среди всех компактов в $\mathbb{C}$, в дополнении к которьм выделяется однозначная ветвь квадратного корня $\sqrt{h(z)} ; S=\bigcup_{j=1}^{\mathrm{g}+1} S_{j}$, $S_{j}=\operatorname{arc}\left(e_{2 j-1}, e_{2 j}\right)$ (дуги $S_{j}$, вообше говоря, могут пересекаться; как хорошо известно, компакт минимальной емкости $S$ допускает полную характеризацию в терминах некоторого условия "симметрии"); под $\sqrt{h(\zeta)}, \zeta \in S$, понимаются "верхние" граничные значения на $S$ той ветви корня, которая определяется условием $\sqrt{h(z)} / z^{\mathrm{g}+1} \rightarrow 1$ при $z \rightarrow \infty$, т.е. граничные значения на “левом" берегу каждой дуги $S_{j}$, ориентированной от $e_{2 j-1}$ к $e_{2 j}$; кроме того, комплекснозначная функция $\rho$ не имеет нулей и удовлетворяет на $S$ условию Гёльдера с некоторым показателем $\alpha \leqslant 1$ (будем говорить, что $\rho$ Н-непрерывна на $S$ и писать $\rho \in \mathrm{H}(\alpha ; S), 0<\alpha \leqslant 1)$. Такую функшию $\rho$ будем назьвать допустимылм весом. В частном случае, когда $\rho(\zeta)$ - рациональная функция переменной $\zeta(\rho \in \mathbb{C}(\zeta))$, не имеющая на $S$ ни нулей, ни полюсов, функция $\widehat{\rho}(\cdot ; h)$ - гиперэллиптическая функция, определенная на гиперэллиптической римановой поверхности $\mathfrak{R}=\mathfrak{R}(h)$ рода $\mathrm{g}=\mathrm{g}(h)$, заданной уравнением $w^{2}=h(z)$ : $\widehat{\rho}(z ; h)=r_{1}(z) w+r_{2}(z)$, где $r_{1}, r_{2} \in \mathbb{C}(z)$; тем самьм, $\widehat{\rho}(z ; h) \in \mathbb{C}(z, w)$. Наконец, отметим, что в соответствии с выбранной нами в $\overline{\mathbb{C}} \backslash S$ ветвью функции $\sqrt{h}$ марковскому случаю положительной меры $\mu$ с $S_{\mu}=S$ соответствует такой вес $\rho$ с носителем на $\mathbb{R}$, для которого вьполняется неравенство $\rho / i \sqrt{h}>0$ на $S$.

Вопрос о сходимости диагональных и близких к ним последовательностей аппроксимаций Паде функщий вида (0.6) изучался в [35], где дано обобщение на случай произвольного $h \in \mathbb{C}[z]$ четной степени и комплексного веса $\rho \neq 0$ классического anпроксимачионного метода Бернштейна-Сегё [37], основанного на явных формулах для полиномов, ортогональных на отрезке $[-1,1]$ относительно веса $\rho$ такого, что $1 / \rho \in \mathbb{R}[x]$; в этом случае $\widehat{\rho}(z)=r_{1}(z) w+r_{2}(z)$, где $r_{1}, r_{2} \in \mathbb{C}(z)$, а $w^{2}=z^{2}-1$. В [35] была доказана сходимость по емкости таких аппроксимаций к функции $\widehat{\rho}$ равномерно внутри (на компактных подмножествах) области $D=\overline{\mathbb{C}} \backslash S$ при несколько более обших, чем в настоящей статье, условиях на вес $\rho$, но также в предположении, что компакт минимальной емкости имеет ровно g +1 компоненту связности (т.е. дуги $S_{j}$ попарно не пересекаются); здесь мы накладываем это условие на взаимное расположение точек $e_{1}, \ldots, e_{2 \mathrm{~g}+2}$ как первое условие "общего положения". Как уже отмечалось, использование понятия сходимости по емкости связано с существом дела: хотя "почти все" полюсы диагональных аппроксимаций Паде $[n / n]_{\widehat{\rho}}$ функции $\widehat{\rho}$ "стягиваются" к $S$, конечное число полюсов рациональных функций $[n / n]_{\widehat{\rho}}$ могут иметь предельные точки в $D$ - области голоморфности функции $\widehat{\rho}$. Наличие таких “лишних" полюсов и является по существу единственным препятствием для равномерной сходимости диагональных аппроксимаций Паде функций вида (0.6). Таким образом, вопрос о сушествовании равномерно сходяшейся подпоследовательности апшроксимаций Паде сводится к вопросу о существовании подпоследовательности, по которой эти аппроксимации не имеют полюсов в $D$. В свою очередь, поскольку знаменатели диагональных аппроксимаций Паде функции (0.6) - многочлены, неэрмитово ортогональные на $S$ относительно веса $\rho(\zeta) / \sqrt{h(\zeta)}$, естественным образом приходим к задаче о сильной асимптотике таких ортогональных полиномов - полиномов Паде.

Случай, когда $S$ состоит из нескольких отрезков, - классический: соответствуюшие ортогональные многочлены впервые рассматривались Ахиезером [38] и носят его имя.

В целом же рассматриваемая здесь задача об асимптотике полиномов Паде потре- 
бовала нового подхода, которьй впервые был предложен Наттоллом [39]: опираясь на некоторые изученные частные случаи, он высказал гипотезу, что асимптотику полиномов Паде можно выразить в терминах решения некоторой краевой задачи Римана, поставленной на расширенной комплексной плоскости с разрезами по аналитическим дугам, составляющим компакт минимальной емкости $S$. В [29] нам удалось при некоторых достаточно обших геометрических условиях на расположение точек $e_{1}, \ldots, e_{2 \mathrm{~g}+2}$ (точнее, в случае "общего положения") фактически охарактеризовать асимптотическое поведение полиномов Паде в терминах решения такой краевой задачи Римана. Анализ сушествования и нахождение “явного" вида решения этой задачи дается в [29] в терминах обладаюшего специальньми свойствами решения проблемы Якоби обрашения абелевых интегралов. Наконец, используя это решение задачи Римана, мы выводим сингулярное интегральное уравнение на полиномы Паде, частный случай которого для g $=0$ (т.е. при $S=[-1,1]$ ) был получен Наттоллом [40], [41]. Отметим, что после этих работ Наттолла предпринимались попытки обобшить найденное им сингулярное уравнение на случай произвольного $g \in \mathbb{N}$, в результате чего были получены некоторые версии такого интегрального уравнения для частных случаев $\mathrm{g}=0$ и $\mathrm{g}=1$ (см. [42], где имеются дальнейшие ссылки).

Таким образом, представленньй новьй подход позволил при изучении асимптотики полиномов Паде обойтись без традиционного апшроксимационного метода Бернштейна-Сегё, о котором говорилось вьше.

Класс функций вида

$$
f(z)=\widehat{\rho}(z ; h)+r(z), \quad z \in \overline{\mathbb{C}} \backslash S,
$$

где $h \in \mathbb{C}[z], \operatorname{deg} h=0(\bmod 2)$, множество $S=S(h)$ обладает определенным свойством "симметрии”, функция $\rho \neq 0$ и Н-непрерывна на $S$, а $r \in \mathbb{C}(z) \cap \mathscr{H}(S)$, будем называть классом Наттолла и обозначать через $\mathcal{N}$; если же, кроме того, в $(0.7)$ все нули полинома $h$ лежат на $\mathbb{R}$ и вес $\rho$ удовлетворяет неравенству $\rho /(i \sqrt{h})>0$ на $S$, то будем писать $f \in \mathscr{N}^{\circ}$. Для произвольной функции $f \in \mathscr{N}$ соответствуюшую область $\overline{\mathbb{C}} \backslash S$ будем обозначить через $D=D_{f}$.

Пусть $f$ - ряд $(0.3),\left\{[n / n]_{f}\right\}_{n \in \mathbb{N}}$ - соответствуюшая ему главная диагональ Паде.

Для произвольной точки $a \in D_{f}$ положим: $k_{f}(a) \in \mathbb{Z}_{+}-$число полюсов рациональной функции $[n / n]_{f}$, стремяшихся к точке $a$ при $n \rightarrow \infty ; p_{f}(a)-$ кратность полюса функщии $f$ в этой точке, если $a$ - полюс $f$, и $p_{f}(a)=0$ - в противном случае; тем самым, $\left\{\left(a, p_{f}(a)\right): p_{f}(a) \geqslant 1\right\}$ - дивизор полюсов функции $f \in \mathscr{N}$ в области $D_{f}$.

Для функции $f \in \mathcal{N}^{\circ}$ “обшим положением” будем назьвать ситуацию, когда нули соответствуюшего полинома $h=h(z ; f)$, лежашие на одной прямой, таковы, что числа $\omega_{1}(\infty), \ldots, \omega_{\mathrm{g}+1}(\infty)$ - гармонические меры отрезков $S_{1}, \ldots, S_{\mathrm{g}+1}$ относительно области $D_{f}$, вычисленные в бесконечно удаленной точке - рационально независимы. Нетрудно видеть, что такая ситуащия действительно является "обшим положением" в смысле работы Шталя [43].

"Общее положение" для функщии в классе $\mathscr{N}$ определяется условиями $\left(C_{1}\right)-\left(C_{2}\right)$ :

$\left(C_{1}\right)$ Аналитические дуги $S_{j}, j=1, \ldots, \mathrm{g}+1$, составляющие компакт минимальной емкости $S$, не пересекаются. 
$\left(C_{2}\right)$ Периоды комплексной функции Грина $G_{D}(z, \infty)$, соответствующей компакту минимальной емкости $S$, рационально независимы с числом $2 \pi i$ (напомним, что комплексная функция Грина имеет $2 \mathrm{~g}$ независимых периодов, которые все чисто мнимые).

Основньми результатами второй главы работы являются следуюшие теоремы.

ТЕОРема 3. Пусть ряд (0.3) определяет функиию $f \in \mathscr{N}^{\circ}$ и нули полинома $h=h(z ; f)$, лежащие на одной прямой, находятся в "общем положении". Тогда $p_{f}(a)=k_{f}(a)$ для любой точки $a \in D_{f}$.

ТеОрема 4. Пусть ряд (0.3) определяет функиию $f \in \mathscr{N}$, причем нули полинома $h=h(z ; f)$ находятся в "общем положении" $\left(C_{1}\right)-\left(C_{2}\right)$. Тогда $p_{f}(a)=k_{f}(a)$ для любой точки $a \in D_{f}$.

Таким образом, в условиях теорем 3 и 4 каждьй полюс $a$ функции $f$ в $D$ притягивает ровно $k=p_{f}(a)$ полюсов аппроксимаций Паде $[n / n]_{f}, n \in \mathbb{N}$.

Иначе говоря, диагональные аппроксимации Паде восстанавливают дивизор полюсов находящейся в “общем положении” произвольной мероморфной функции из класса $\mathscr{N}$ или из класса $\mathscr{N}^{\circ}$.

Из метода доказательства теорем 3 и 4 вытекает следуюший (формально более обший) результат.

СлЕДСТВИЕ. В условиях теоремы 3 или 4 главная диагональ Паде восстанавливает дивизор (нулей и полюсов) функции $f$ в $D_{f}$.

Эти результаты являются прямьми следствиями утверждений о существовании для находящейся в “общем положении” произвольной функции из класса $\mathscr{N}^{\circ}$ или $\mathscr{N}$ равномерно сходяшейся в сферической метрике подпоследовательности диагональных аппроксимаций Паде.

ПРЕДЛОЖЕНИЕ 2.1. Если точки $e_{1}, \ldots, e_{2 g+2}$ расположены на одной прямой и числа $\omega_{1}(\infty), \ldots, \omega_{\mathrm{g}+1}(\infty)$ - гармонические меры отрезков $S_{1}, \ldots, S_{\mathrm{g}+1}$ относительно области $D$ - рационально независимь, то для любой функции $f(z)=$ $\widehat{\rho}(z ; h)+r(z)$, где $\rho$ - допустимый в классе $\mathscr{N}^{\circ}$ вес на $S$, а $r \in \mathbb{C}(z) \cap \mathscr{H}(S)$, существует подпоследовательность $\Lambda \subset \mathbb{N}$ такая, что

$$
[n / n]_{f} \rightarrow f, \quad n \rightarrow \infty, \quad n \in \Lambda
$$

равномерно в сферической метрике внутри $D$.

Аналогичный результат имеет место для произвольной $f \in \mathscr{N}$ в случае "общего положения" $\left(C_{1}\right)-\left(C_{2}\right)$.

Отметим, что первьй положительньй результат, касающийся упомянутой ранее гипотезы Бейкера-Гамелля-Уиллса, был получен Шталем [43] для гиперэллиптических функций

$$
f(z)=r_{1}(z) w+r_{2}(z),
$$

$w^{2}=h(z), r_{1}, r_{2} \in \mathbb{C}(z)$, при некотором дополнительном условии на нули и полюсы рациональных функций $r_{1}, r_{2}$ и также в условиях "общего положения". Непосредственно из полученного во второй главе тождества (2.9) вытекает, что "общее положение" из этой работы Шталя эквивалентно используемому в настоящем обзоре. Тем 
самым, из приведенных вьше результатов вытекает, что гипотеза Бейкера-ГамелляУиллса справедлива и для находящейся в “общем положении” произвольной функции из класса Наттолла (0.7), включающего в себя все гиперэллиптические функции.

Наш подход к исследованию равномерной сходимости диагональных аппроксимаций Паде основан на доказательстве формулы сильной асимптотики для знаменателей $Q_{n}$ этих рациональных функций - полиномов Паде.

В свою очередь, изучение асимптотических свойств полиномов Паде основано на исследовании некоторой краевой задачи Римана для многосвязной области $D$. При вьполнении условия $\left(C_{1}\right) \quad D=\overline{\mathbb{C}} \backslash S$ есть g-связная область в $\overline{\mathbb{C}}$. Через $S_{j}^{+}, S_{j}^{-}$, $j=1, \ldots, \mathrm{g}+1$, будем обозначать соответственно "верхний" и "нижний" берега дуг $S_{j}=\operatorname{arc}\left(e_{2 j-1}, e_{2 j}\right), j=1, \ldots, \mathrm{g}+1$, составляюших $S$ и ориентированных согласованно с ориентацией $S$ как границы области $D$ ("верхний" берег остается "слева" при движении от $e_{2 j-1}$ к $\left.e_{2 j}\right)$. Через $\mathscr{C}(D)$ обозначим класс всех функций, мероморфных в $D=\overline{\mathbb{C}} \backslash S$ и Н-непрерьвно продолжимых на $S^{+} \cup S^{-}$. Предельные значения функций такого класса на $S^{+}$и $S^{-}$будем обозначать соответственно при помощи "+" и "-".

Пусть на $S$ задана комплекснозначная Н-непрерывная функция $\rho \neq 0$ (т.е. допустимый вес). Для фиксированного $n \in \mathbb{N}, n \geqslant \mathrm{~g}$, рассмотрим следующую краевую задачу Римана.

ЗАДАчА $(\mathscr{R})$. Найти пару функций $\varphi_{n}, \psi_{n} \in \mathscr{C}(D)$ таких, что

(i) $\varphi_{n}$ имеет полюс порядка $n$ в точке $z=\infty$ и не имеет ни других полюсов, ни нулей в $D$;

(ii) $\psi_{n}$ имеет нуль кратности $n-\mathrm{g}$ в точке $z=\infty$ и еще ровно g “свободных" нулей в $D^{*}=D \backslash\{\infty\} ;$ других нулей или полюсов функция $\psi_{n}$ не имеет;

(iii) на множестве $S$ выполняются краевые условия: $\rho \varphi_{n}^{+}=\psi_{n}^{-}, \rho \varphi_{n}^{-}=\psi_{n}^{+}$.

Пару таких функций $\varphi_{n}=\varphi_{n}(z ; \rho), \psi_{n}=\psi_{n}(z ; \rho)$, нормированных условием $\varphi_{n}(z) / z^{n} \rightarrow 1$ при $z \rightarrow \infty$, назовем решением задачи $(\mathscr{R})$.

Как уже говорилось вьше, здесь используется новый подход к изучению асимптотических свойств полиномов Паде $Q_{n}(z ; \widehat{\rho})$, основанньй на свойствах решения задачи $(\mathscr{R})$ : нами фактически доказано, что при условии $\left(C_{1}\right)$ достаточным условием существования по некоторой подпоследовательности сильной асимптотики полиномов Паде является разрешимость по этой подпоследовательности задачи $(\mathscr{R})$. Точнее, имеет место следующее

ПРЕДЛОЖЕНИЕ 2.4. Если для некоторой последовательности $\Lambda \subset \mathbb{N}$ задача $(\mathscr{R})$ имеет решение $\varphi_{n}, \psi_{n}, n \in \Lambda$, причем все предельные точки "свободных" нулей функиий $\psi_{n}$ принадлежат области $D^{*}=D \backslash\{\infty\}$, mо $\operatorname{deg} Q_{n}=n$ для достаточно больших $n \in \Lambda$ и при нормировке полиномов Паде условием $Q_{n}(z ; \widehat{\rho})=z^{n}+\cdots$ для $n \in \Lambda$ имеем:

(i) $Q_{n}(z ; \widehat{\rho})=\varphi_{n}(z ; \rho)(1+o(1)), n \rightarrow \infty$, равномерно внутри области $D$;

(ii) $Q_{n}(\zeta ; \widehat{\rho})=\varphi_{n}^{+}(\zeta ; \rho)+\varphi_{n}^{-}(\zeta ; \rho)+o(1)$ равномерно на $S$;

(iii) если вес $\rho$ аналитичен на каждой дуге $S_{j}$, то в (i)-(ii) имеем: $о(1)=o\left(\delta^{n}\right)$, $0<\delta<1 ;$ при $\rho \in \mathrm{H}(\alpha ; S)$ в (i)-(ii) имеем: $o(1)=O\left((\log n) / n^{\alpha}\right)$.

Задачу $(\mathscr{R})$ удается исследовать с нужным для нас результатом пока только для двух случаев, соответствуюших обшему положению в классе $\mathscr{N}^{\circ}$ или в классе $\mathscr{N}$. 
На самом деле асимптотическое поведение полиномов Паде фактически полностью описыв вается в терминах решения краевой задачи Римана $(\mathscr{R})$ при условии, что это решение понимается в несколько более широком, чем ранее, смысле. А именно, если предположить, что для каждого $n$, принадлежашего некоторой подпоследовательности $\Lambda^{\prime}$, сушествует пара функций $\varphi_{n}, \psi_{n}$ таких, что на $S$ выполняются краевые условия (iii) задачи $(\mathscr{R})$ и

$\left(\mathrm{i}^{\prime}\right)$ функция $\varphi_{n}$ имеет полюс порядка $n$ в точке $z=\infty$ и ровно $k \leqslant \mathrm{~g}$ "свободных" нулей в $D^{*}=D \backslash\{\infty\}$,

(ii') $\psi_{n}$ имеет нуль кратности $n-\mathrm{g}$ в точке $z=\infty$ и еще ровно $\mathrm{g}-k$ "свободных" нулей в $D^{*}=D \backslash\{\infty\}$,

то для таких функщий $\varphi_{n}$ и $\psi_{n}$ при условии, что множества свободных нулей этих двух функций не пересекаются, по подпоследовательности $\Lambda^{\prime}$ имеет место аналог предложения 2.4. Отсюда уже легко вытекает

ПРЕДЛОЖЕНИЕ 2.6. В случае “общего положсения" (I) (см. Гл. II, $\S 1$, п. 1.2) множество предельных точек полюсов диагональных аппроксимачий Паде совпадает с $\widehat{S_{\mu}}=[a, b]$, а в случае $(\mathrm{II})$ - со всей римановой сферой $\overline{\mathbb{C}}$.

4. Третья глава обзора посвящена одному из наиболее естественных обобщений классической конструкции Паде - апшроксимациям Паде ортогональных разложсений, некоторые свойства которых рассматривались ранее в связи с приложениями подобных рациональных функций [44], [45]. Вопросы сходимости таких рациональных аппроксимаций впервые изучены автором настоящей работы, в частности, для строчных последовательностей апшроксимаций Паде ортогональных разложений получены аналоги классической теоремы Монтессу де Болора [46]-[49]. В [50] исследованы также аппроксимации Паде для разложений в ряды по полиномам Фабера.

Пусть на отрезке $\Delta=[-1,1]$ задана положительная борелевская мера $d \sigma(x)$ такая, что $\sigma^{\prime}(x)=d \sigma / d x>0$ п.в. на $\Delta ;\left\{P_{k}(x, d \sigma)\right\}_{n=0}^{\infty}-$ соответствуюшая система полиномов с положительньми старшими коэффициентами, ортонормированных относительно меры $d \sigma(x)$ :

$$
\int_{\Delta} P_{j}(x) P_{k}(x) d \sigma(x)= \begin{cases}1, & j=k \\ 0, & j \neq k\end{cases}
$$

$P_{k}(x)=P_{k}(x ; \sigma)=\alpha_{k} x^{n}+\cdots, \alpha_{k}>0, n=0,1, \ldots$ Наиболее важные примеры ортогональных полиномов такого типа: полиномы Лежандра $(d \sigma(x) \equiv d x)$ и Чебышёва $\left(d \sigma(x) \equiv d x /\left(\pi \sqrt{1-x^{2}}\right)\right)$.

Произвольной вешественной функции $f(x)$, суммируемой на отрезке $\Delta$ по мере $d \sigma(x)$, соответствует (формальный или сходящийся) ряд Фурье по системе $\left\{P_{k}(x)\right\}$ :

$$
f(x) \sim \sum_{k=0}^{\infty} c_{k} P_{k}(x), \quad c_{k}=c_{k}(f)=\int_{\Delta} f(x) P_{k}(x) d \sigma(x)
$$

Существуют (см. [44], [45], а также [13]) следуюшие два варианта определения рациональных аппроксимаций ряда (0.8), аналогичных классическим аппроксимациям Паде степенного ряда (0.1). 
Для фиксированных неотрицательных целых чисел $n$ и $m$ аппроксимацией Паде типа $(n, m)$ ряда (0.8) (или обобщенной аппроксимацией Паде функиии $f$, соответствующей системе $\left.\left\{P_{k}(x)\right\}\right)$ назовем:

А) рациональную функцию $R_{n, m} \in \mathscr{R}_{n, m}$ такую, что $R_{n, m}=p / q$, где $p, q \in \mathbb{C}[z]$, $\operatorname{deg} p \leqslant n, \operatorname{deg} q \leqslant m, q \not \equiv 0$ и

$$
(q f-p)(x) \sim \sum_{k=n+m+1}^{\infty} a_{k}^{(n, m)} P_{k}(x)
$$

В) рациональную функцию $F_{n, m} \in \mathscr{R}_{n, m} \cap \mathscr{H}(\Delta)$ такую, что

$$
\left(f-F_{n, m}\right)(x) \sim \sum_{k=n+m+1}^{\infty} b_{k}^{(n, m)} P_{k}(x) .
$$

Для нахождения функции $R_{n, m}$ (точнее, полиномов $p$ и $q$ ) требуется решить систему из $m$ линейны $x$ однородных уравнений относительно $m+1$ неизвестных величин коэффициентов полинома $q$. Таким образом, аппроксимация Паде типа $(n, m)$ в смысле варианта A) всегда существуеm, нетрудно однако заметить, что она, вообще говоря, не единственна. Вместе с тем, непосредственно из (0.9) вытекает, что если любой полином $q$, удовлетворяющий $(0.9)$, имеет степень ровно $m$, то рациональная функция $R_{n, m}$ определена однозначно: $R_{n, m}=R_{n, m}(f)$.

Для сушествования функции $F_{n, m}$ требуется, чтобы сушествовало решение вытекающей из (0.10) нелинейной системы из $n+m+1$ уравнения относительно $n+m+2$ неизвестных величин, что оказывается возможным не всегда. Тем самым, аппроксимация Паде типа $(n, m)$ ряда (0.8) в смысле варианта В) ( "нелинейная" апшроксимашия Паде) определена не всегда. Однако если функщия $F_{n, m}$ сушествует, то она $е \partial u н-$ ственна.

В отличие от классического случая, указанные варианты определения приводят, вообще говоря, к различным рациональным функщиям. Можно, например, подобрать последовательность чисел $\left\{c_{k}\right\}$ так, что нелинейные аппроксимации Паде $F_{n, 1}(z)$ типа $(n, 1)$ функции

$$
f(z)=\frac{1}{z-2}+\sum_{k=0}^{\infty} c_{k} T_{k}(z),
$$

где $T_{k}(z)$ - полиномы Чебышёва первого рода, ортонормированные на $\Delta=[-1,1]$ относительно веса $1 / \pi \sqrt{1-x^{2}}$, всегда существуют и не совпадают с соответствуюшими аппроксимациями $R_{n, 1}$ ни при каких $n$ (см. [50]).

Подчеркнем еше раз, что ранее такие аппроксимации рассматривались только лишь как формальные конструкции, позволяюшие достаточно хорошо решать задачу численного приближения и представления заданной рядом (0.8) функции. Вопросы сходимости этих рациональных аппроксимаций автором обзора были рассмотрены впервые.

Более точно, речь идет об исследовании сходимости аналогов строчныл последовательностей рациональных аппроксимаций ортогональных разложений, прежде всего - об обобшении на каждый из рассмотренных выше случаев А) и В) классической теоремы Монтессу де Болора. 
Для любого $\rho>1$ положим $\Gamma_{\rho}=\{z \in \mathbb{C}:|\varphi(z)|=\rho\}$, где $\varphi-$ функция, конформно отображающая внешность отрезка $\Delta=[-1,1]$ на внешность единичного круга так, что $\varphi(\infty)=\infty, \varphi^{\prime}(\infty)>0\left(\varphi(z)=z+\sqrt{z^{2}-1}\right.$, где ветвь корня выбрана так, что $|\varphi(z)|>1$ вне $\Delta ; \Gamma_{\rho}-$ эллипс с фокусами в точках \pm 1 и суммой полуосей, равной $\left.\rho\right)$. Внутренность эллипса $\Gamma_{\rho}$ обозначим через $D_{\rho}$; области $D_{\rho}, \rho \in(1,+\infty)$, будем называть каноническими (относительно $\Delta$ ). Функция $f$ предполагается голоморфной на $\Delta$ (тем самым, и в некоторой окрестности $\Delta$ ). Пусть $D_{0}(f)$ - максимальная каноническая область, в которую $f$ продолжается как голоморфная функция. Тогда $f$ может быть разложена в ряд Фурье (0.8) по системе $\left\{P_{k}(x)\right\}$, равномерно сходящийся к $f$ внутри (на компактных подмножествах) $D_{0}(f)$. Аналогично, для любого $m \in \mathbb{N}$ через $D_{m}(f)$ обозначим максимальную каноническую область, в которую $f$ продолжается как мероморфная функция, число полюсов которой $\leqslant m$; имеем $D_{m}(f)=D_{\rho_{m}}$, $\rho_{m}=\rho_{m}(f)$.

Основньми результатами третьей главы являются следуюшие теоремы 5 и 6 , обобщающие классический результат Монтессу де Болора.

ТеОРема 5. Если функиия $f$ имеет ровно $m$ полюсов в области $D_{m}(f)$, то

$$
R_{n, m} \rightarrow f, \quad n \rightarrow \infty
$$

равномерно в сферической метрике внутри $D_{m}(f)$.

Тем самым, для достаточно больших $n$ рациональные функции $R_{n, m}$ имеют ровно $m$ конечных полюсов (т.е. полином $q=q_{n, m}(f)$ имеет степень ровно $m$ ); при $n \rightarrow \infty$ конечные полюсы $R_{n, m}$ стремятся к полюсам $f$ в $D_{m}(f)$, причем каждый полюс $f$ “притягивает" столько полюсов $R_{n, m}$, какова его кратность; в условиях теоремы 5 для достаточно больших $n$ сушествует единственная аппроксимация Паде $R_{n, m}=$ $R_{n, m}(f)$ функции $f$. Скорость сходимости последовательности $\left\{R_{n, m}\right\}$ к $f$ характеризуется неравенством

$$
\varlimsup_{n \rightarrow \infty}\left|\left(f-R_{n, m}\right)(z)\right|^{1 / n} \leqslant \frac{|\varphi(z)|}{\rho_{m}}<1 .
$$

ТеОРема 6. Если функция $f$ имеет ровно $m$ полюсов в области $D_{m}(f)$, то при каждом достаточно большом $n$ существует обобщенная аппроксимация Паде $F_{n, m}$ muna $(n, m)$ функиии $f u$

$$
F_{n, m} \rightarrow f, \quad n \rightarrow \infty
$$

равномерно в сферической метрике внутри $D_{m}(f)$.

В условиях теоремы 6 скорость сходимости последовательности $F_{n, m} \mathrm{k} f$ характеризуется неравенством, аналогичным (0.11).

Из доказательства теорем 5 и 6 вытекает, что конечные полюсы рациональных функций $R_{n, m}$ и $F_{n, m}$ стремятся к полюсам $f$ в $D_{m}(f)$ со скоростью геометрической прогрессии.

Оказалось, что такое свойство полюсов функций $R_{n, m}$ является характеристическим для $m$-мероморфного продолжения $f$. 
ПРЕДЛОЖЕНИЕ. Для функиии $f$, голоморфной на $\Delta$, следующие два утверждения әквивалентны:

$1^{\circ}$. Функция $f$ имеет в области $D_{m}(f)$ ровно $m$ полюсов.

$2^{\circ}$. Рациональные функции $R_{n, m}$ для всех достаточно больших $n$ имеют ровно $m$ конечных полюсов, и существует полином $\omega_{m}(z)=z^{m}+\cdots$ степени $m$ такой, что при нормировке $q_{n, m}(z)=z^{m}+\cdots$

$$
\varlimsup_{n \rightarrow \infty}\left\|\omega_{m}-q_{n, m}\right\|^{1 / n}=\delta<1
$$

При әтом (если выполнено какое-либо из условий $1^{\circ}$ и $2^{\circ}$ ) полюсы функции $f \boldsymbol{\theta}$ $D_{m}(f)$ совпадают с нулями $z_{1}, \ldots, z_{m}$ полинома $\omega_{m}(z) u$

$$
\rho_{m}(f)=\max _{1 \leqslant j \leqslant m} \frac{\left|\varphi\left(z_{j}\right)\right|}{\delta}
$$

(под $\|\cdot\|$ здесь понимается норма соответствующего полинома в пространстве коэффициентов).

В заключение отметим, что некоторые вопросы сходимости диагональныл аппроксимаций Паде ортогональных разложений изучены в работах [51]-[53].

\section{Глава I.}

\section{Полюсы строк таблицы Паде и особые точки аналитической функции}

В этой главе исследуется связь между асимптотическим поведением конечных полюсов строчных последовательностей аппроксимаций Паде степенного ряда и особыми точками продолженной функции как внутри, так и на границе соответствующего круга мероморфности. Главным результатом является обобщение классической теоремы Фабри “об отношении” на случай $m$-й строки таблицы Паде степенного ряда. В основе доказательства обобщенного варианта теоремы Фабри лежит модификация классического метода Агмона, учитывающего связь между опорной функцией и индикатором для соответственно нижней и верхней функций, ассоциированных по Борелю. Основные результаты этой главы опубликованы в работах автора [24], [25].

\section{§ 1. Формулировка основных результатов}

Пусть

$$
f(z)=\sum_{k=0}^{\infty} c_{k} z^{k}
$$

- произвольньй степенной ряд, $R_{0}=R_{0}(f) \geqslant 0$ - радиус его сходимости. Нетрудно видеть, что для любых неотрицательных целых чисел $n, m$ существует пара полиномов $p, q \in \mathbb{C}[z]$ таких, что $\operatorname{deg} p \leqslant n, \operatorname{deg} q \leqslant m, q \not \equiv 0$, и выполняется следующее условие:

$$
(q f-p)(z)=O\left(z^{n+m+1}\right), \quad z \rightarrow 0
$$


Полиномы $p, q$, удовлетворяюшие соотношению (1.2), определяются, вообще говоря, не единственным образом, однако их отношение $p / q$ задает единственную рациональную функцию - аппроксимацию Паде $[n / m]_{f}=p / q$ типа $(n, m)$ ряда (1.1), доставляюшую максимально возможньй порядок касания в классе $\mathscr{R}_{n, m}=\left\{r \in \mathbb{C}(z): r=\left(a_{0}+a_{1} z+\cdots+a_{n} z^{n}\right) /\left(b_{0}+b_{1} z+\cdots+b_{m} z^{m}\right)\right\}$ к этому ряду в начале координат.

Зафиксируем произвольное натуральное число $m$ и рассмотрим $m$-ю строку таблищы Паде ряда $(1.1):[n / m]_{f}(z), n=0,1, \ldots$.

Пусть $\mathscr{P}_{n}=\left\{\zeta_{n, 1}, \ldots, \zeta_{n, m_{n}}\right\}, 0 \leqslant m_{n} \leqslant m,-$ множество конечных полюсов рациональной функции $[n / m]_{f}$ (каждьй полюс вьписывается столько раз, какова его кратность). Введем следуюшие характеристики асимптотического поведения последовательности множеств $\mathscr{P}_{n}, n=0,1, \ldots$, в окрестности произвольной точки $a \in \mathbb{C}^{*}=\mathbb{C} \backslash\{0\}: \mu(a)$ - число точек множества $\mathscr{P}_{n}$, стремящихся к точке $a$ со скоростью геометрической прогрессии при $n \rightarrow \infty ; \mathscr{P}=\left\{a \in \mathbb{C}^{*}: \mu(a) \geqslant 1\right\}$;

$$
\Delta(a)=\varlimsup_{n \rightarrow \infty} \prod_{j=1}^{m_{n}}\left|a ; \zeta_{n, j}\right|^{1 / n}
$$

где $|\cdot ; \cdot|-$ расстояние между точками в сферической метрике.

Очевидно, $\mu(a) \geqslant 1 \Longleftrightarrow \Delta(a)<1$ и

$$
\Delta(a)=\varlimsup_{n \rightarrow \infty}\left|Q_{n}(a)\right|^{1 / n}
$$

где $Q_{n}$ - “сферически” нормированные знаменатели рациональных функций $[n / m]_{f}$ :

$$
Q_{n}(z)=\prod_{\left|\zeta_{n, j}\right| \leqslant 1}\left(z-\zeta_{n, j}\right) \cdot \prod_{\left|\zeta_{n, j}\right|>1}\left(1-\frac{z}{\zeta_{n, j}}\right)
$$

Если $R_{0}>0$, то через $D_{m}=D_{m}(f):|z|<R_{m}$ обозначим максимальный открытый круг с центром в точке $z=0$, в которьй функция $f(z),|z|<R_{0}$, продолжается как мероморфная функция, имеюшая $\leqslant m$ полюсов $\left(R_{m}=R_{m}(f)\right.$ - радиус $m$-мероморфности функции $f$ ). Если $R_{0}=0$, то полагаем $R_{m}=0$; тем самым, утверждение о положительности $R_{m}$ означает, в частности, что ряд (1.1) определяет функцию $f$, голоморфную в точке $z=0$.

В терминах характеристик $\mu$ и $\Delta$ А. А. Гончар $[16]$ полностью описал $m$-мероморфное продолжение ряда (1.1).

ТеОРема ГОНЧАРА. Пусть $f-$ яяд (1.1), $m \in \mathbb{N}$ и $a \neq 0-$ фиксированная точка комплексной плоскости. Следующие утверждения әквивалентны:

$1^{\circ} . R_{m}>|a|$ u $f$ имеет полюс в точке $a$;

$2^{\circ} . \Delta(a)<1$ (или, что то же самое, $\mu(a) \geqslant 1$ ).

При әтом (если выполнено какое-либо из условий $1^{\circ}$ и $2^{\circ}$ ) кратность полюса $f \boldsymbol{\theta}$ точке а равна $\mu($ а) и справедлива формула

$$
R_{m}=\frac{|a|}{\Delta(a)} .
$$


Тем самым, если $d_{m}=\left\{\left(a_{1}, \nu_{1}\right), \ldots,\left(a_{s}, \nu_{s}\right)\right\}-$ дивизор полюсов функции $f$ в круге $D_{m}\left(a_{j}\right.$ - полюсы $f$ в $D_{m}, \nu_{j} \geqslant 1$ - кратность полюса в точке $a_{j} ;\left|d_{m}\right|=\nu_{1}+$ $\cdots+\nu_{s} \leqslant m$ - число полюсов $f$ в $\left.D_{m}\right)$, то из теоремы Гончара вытекает, что $n p u$ любом $m \in \mathbb{N}$ дивизор $d_{m}=\{(a, \mu(a)): a \in \mathscr{P}\}$. Утверждение классической теоремы Монтессу по существу состоит в том, что эта формула для вычисления дивизора функции $f$ справедлива при $m \in \mathbb{N}$ таком, что $\left|d_{m}\right|=m$. Отметим, что при доказательстве своей теоремы Монтессу де Болора в сушественной степени опирался на полученные ранее Адамаром [15] явные формулы для радиусов $R_{m}$, которые фактически основаны на анализе поведения полюсов двух соседних строк таблицы Паде ряда (1.1). В отличие от этого, в приведенном вьше результате Гончара и радиус $R_{m}$ круга $m$-мероморфности функции $f$, и дивизор $d_{m}$ ее полюсов в этом круге характеризуются поведением полюсов только $m$-й строки таблицы Паде (при любом $m \in \mathbb{N}$ ).

Естественным образом возникает следующий вопрос: какие вьводы можно сделать о функции $f$ в целом, если известно, что конечные полюсы $m$-й строки таблицы Паде стремятся к некоторым точкам в комплексной плоскости без какого-либо предварительного предположения о скорости этой сходимости.

Основньми результатами первой главы являются следуюшие теоремы.

TeOpema 1. Пусть $\operatorname{dist}\left(a, \mathscr{P}_{n}\right) \rightarrow 0$ при $n \rightarrow \infty$ для некоторой точки $a \neq 0$ плоскости $\mathbb{C}$. Тогда $R_{m} \geqslant|a|$. Если при этом $|a|<R_{m}$, то а - полюс функиии $f$.

Теорема 2. Пусть для всех достаточно больших $n$ рациональные функиии $[n / m]_{f}$ имеют ровно $m$ конечных полюсов $\zeta_{n, 1}, \zeta_{n, 2}, \ldots, \zeta_{n, m}$, причем

$$
\zeta_{n, j} \rightarrow a_{j} \in \mathbb{C}^{*}, \quad n \rightarrow \infty, \quad j=1,2, \ldots, m,
$$

ฉдe

$$
0<\left|a_{1}\right| \leqslant \cdots \leqslant\left|a_{\mu-1}\right|<\left|a_{\mu}\right|=\cdots=\left|a_{m}\right|=R .
$$

Тогда:

$1^{\circ} . R_{\mu-1}(f)=\cdots=R_{m-1}(f)=R$ и все точки $a_{1}, \ldots, a_{\mu-1}$ и только они полюсы функции $f$ в круге $|z|<R$.

$2^{\circ}$. Все точки $a_{\mu}, \ldots, a_{m}$ - особье точки функиии $f$ на граниче круга $|z|<R$.

Для первой строки таблицы Паде единственный конечный полюс $\zeta_{n}$ рашиональной функции $[n / 1]_{f}(z)$ вычисляется по формуле $\zeta_{n}=c_{n} / c_{n+1}$ при условии, что $c_{n} \cdot c_{n+1} \neq 0$. Таким образом, соотношение $\zeta_{n} \rightarrow a \in \mathbb{C}^{*}$ эквивалентно тому, что $c_{n} / c_{n+1} \rightarrow a$ при $n \rightarrow \infty$ и мы оказываемся в условиях классической теоремы Фабри "об отношении". Тем самьм, теорема 2 обобшает эту теорему Фабри на случай $m$-й строки таблицы Паде.

\section{§. Доказательство теоремы 1}

2.1. Всюду в этой главе мы будем придерживаться введенных вьше обозначений; индекс $m$ будем как правило опускать. Положим для краткости $f_{n}:=[n / m]_{f}$ и пусть $f_{n}=P_{n} / Q_{n}$, где полиномы $P_{n}$ и $Q_{n}$ не имеют общих нулей; тогда множество $\mathscr{P}_{n}=$ 
$\left\{\zeta_{n, 1}, \ldots, \zeta_{n, m_{n}}\right\}$ совпадает с множеством нулей полинома $Q_{n}$. Полиномы $Q_{n}$ нормируются условием (1.3). При такой нормировке для любого компакта $K \subset \mathbb{C}$ имеем

$$
\left\|Q_{n}\right\|_{K}:=\max _{z \in K}\left|Q_{n}(z)\right|<C, \quad n=1,2, \ldots,
$$

где $C=C(K)<+\infty$ не зависит от $n$. В дальнейшем через $C_{1}, C_{2}, \ldots$ обозначаются положительные величины, также не зависяшие от $n$.

Хорошо известно (см., например, [16]), что имеет место следующее соотношение:

$$
\left(P_{n+1} Q_{n}-P_{n} Q_{n+1}\right)(z)=A_{n} z^{n+m+1-\lambda_{n}}, \quad n=1,2, \ldots,
$$

где $0 \leqslant \lambda_{n} \leqslant m$; заметим, что величина $A_{n}$ полностью определена этим соотношением. Следовательно,

$$
\left(f_{n+1}-f_{n}\right)(z)=\frac{A_{n} z^{n+m+1-\lambda_{n}}}{\left(Q_{n} Q_{n+1}\right)(z)}, \quad n=1,2, \ldots,
$$

и сходимость последовательности $\left\{f_{n}(z)\right\}$ равносильна сходимости ряда

$$
\sum_{n=1}^{\infty} \frac{A_{n} z^{n+m+1-\lambda_{n}}}{\left(Q_{n} Q_{n+1}\right)(z)}, \quad 0 \leqslant \lambda_{m} \leqslant m .
$$

В [16] доказано, что последовательность $\left\{f_{n}(z)\right\}$ сходится по емкости внутри круга $|z|<R=R_{m}$ к функции $f(z)$ и

$$
\frac{1}{R}=\varlimsup_{n \rightarrow \infty}\left|A_{n}\right|^{1 / n} .
$$

2.2. Докажем первое утверждение теоремы: $е с л u \operatorname{dist}\left(a, \mathscr{P}_{n}\right) \rightarrow 0$ npu $n \rightarrow \infty$ для некоторой точки $a \neq 0$ плоскости $\mathbb{C}$, то $R \geqslant|a|$. Предположим противное: $R<|a|$. Фиксируем $\rho, R<\rho<|a|$. Тогда из (1.6) получаем

$$
\varlimsup_{n \rightarrow \infty}\left|A_{n} \rho^{n}\right|^{1 / n}=\frac{\rho}{R}>1 .
$$

Следовательно, $\overline{\lim }_{n \rightarrow \infty}\left|A_{n} \rho^{n}\right|=\infty$, поэтому существует бесконечная последовательность $\Lambda=\Lambda(\rho) \subset \mathbb{N}$ такая, что при $n \in \Lambda$ имеем:

$$
\left|A_{n} \rho^{n}\right|=\max _{1 \leqslant k \leqslant n}\left|A_{k} \rho^{k}\right| .
$$

Рассмотрим разность

$$
\left(f_{n}-f_{1}\right)(z)=\sum_{k=1}^{n-1} \frac{A_{k} z^{k+m+1-\lambda_{k}}}{\left(Q_{k} Q_{k+1}\right)(z)},
$$

где $n \in \Lambda, z \in \mathbb{C} \backslash \mathscr{P}, \mathscr{P}=\bigcup_{n=1}^{\infty} \mathscr{P}$. Имеем

$$
\left(f_{n}-f_{1}\right)(z)=\sum_{k=1}^{n-1} \frac{A_{k} \rho^{k}(z / \rho)^{k} z^{m+1-\lambda_{k}}}{\left(Q_{k} Q_{k+1}\right)(z)}=A_{n} z^{n} \sum_{k=1}^{n-1} \frac{A_{k} \rho^{k}}{A_{n} \rho^{n}}\left(\frac{z}{\rho}\right)^{k-n} \frac{z^{m+1-\lambda_{k}}}{\left(Q_{k} Q_{k+1}\right)(z)},
$$


$n \in \Lambda, z \in \mathbb{C} \backslash \mathscr{P}$. Следовательно,

$$
\left|\left(f_{n}-f_{1}\right)(z)\right| \leqslant\left|A_{n} z^{n}\right| \sum_{k=1}^{n-1}\left|\frac{z}{\rho}\right|^{k-n} \frac{|z|^{m+1-\lambda_{k}}}{\left|\left(Q_{k} Q_{k+1}\right)(z)\right|},
$$

$n \in \Lambda, z \in \mathbb{C} \backslash \mathscr{P}$. Выберем $r>0$ так, чтобы $2 r$-окрестность $U_{2 r}(a)$ точки $a$ лежала во внешности окружности $|z|=\rho$ (это возможно, поскольку $\rho<|a|)$. Тогда для всех $z \in U_{2 r}(a)$ имеем: $|z / \rho| \geqslant 1 / \delta$, где $0<\delta<1$. Следовательно,

$$
\left|\left(f_{n}-f_{1}\right)(z)\right| \leqslant c_{1}\left|A_{n} z^{n}\right| \sum_{k=1}^{n-1} \frac{\delta^{n-k}}{\left|\left(Q_{k} Q_{k+1}\right)(z)\right|}=C_{1}\left|A_{n} z^{n}\right| \sum_{k=1}^{n-1} \frac{\delta^{k}}{\left|\omega_{k}(z)\right|},
$$

$n \in \Lambda, z \in U_{2 r}(a) \backslash \mathscr{P}$, где мы положили $\omega_{k}(z)=\left(Q_{n-k} Q_{n-k+1}\right)(z), \operatorname{deg} \omega_{k} \leqslant 2 m$, $k=1,2, \ldots, n-1$.

Выберем теперь $\varepsilon>0$ так, чтобы вьполнялось соотношение:

$$
\varepsilon \sum_{k=1}^{\infty} \frac{1}{k^{2}}=\frac{2 r}{3}<r
$$

Фиксируем $n \in \Lambda$. При каждом $k=1,2, \ldots, n-1$ обозначим через $V_{k, \varepsilon}$ множество, состоящее из $\varepsilon /\left(4 m k^{2}\right)$-окрестностей нулей полинома $\omega_{k}$; положим $V_{\varepsilon}^{n}=\bigcup_{k=1}^{n-1} V_{k, \varepsilon}$. Сумма диаметров кругов, входящих в множество $V_{\varepsilon}^{n}$, не превосходит величины $\varepsilon \sum_{k=1}^{n-1} 1 / k^{2}<r$. Поэтому найдется окружность $\gamma_{n}$ с центром в точке $a$ радиуса $r_{n}$, $r<r_{n}<2 r$, которая не пересекается с множеством $V_{\varepsilon}^{n}$. Нетрудно видеть, что для всех $z \in \gamma_{n}, k=1,2, \ldots, n-1$ имеет место неравенство

$$
\left|\omega_{k}(z)\right| \geqslant C_{2}\left(\frac{\varepsilon}{4 m k^{2}}\right)^{2 m}
$$

где величина $C_{2}>0$ не зависит ни от $k$, ни от $n$.

Следовательно, из (1.7) получаем

$$
\begin{aligned}
\left|\left(f_{n}-f_{1}\right)\right| & \leqslant C_{3}\left|A_{n} z^{n}\right|\left(\frac{4 m}{\varepsilon}\right)^{2 m} \sum_{k=1}^{n-1} \delta^{k} k^{4 m} \\
& \leqslant C_{4}\left|A_{n} z^{n}\right| \sum_{k=1}^{\infty} \delta^{k} k^{4 m}=C_{5}\left|A_{n} z^{n}\right|, \quad z \in \gamma_{n}, \quad n \in \Lambda .
\end{aligned}
$$

Так как $\left|A_{n} z^{n}\right| \rightarrow \infty$ на $U_{2 r}(a)$ при $n \rightarrow \infty, n \in \Lambda$, то из (1.8) вытекает неравенство

$$
\left|f_{n}(z)\right| \leqslant C_{6}\left|A_{n} z^{n}\right|, \quad z \in \gamma_{n}, \quad n \in \Lambda .
$$

Умножая обе части (1.9) на $Q_{n}(z)$, используя (1.4) и принцип максимума модуля для голоморфных функций, получаем оценку

$$
\left|\frac{P_{n}(z)}{A_{n} z^{n}}\right| \leqslant C_{7}, \quad z \in U_{r}(a), \quad n \in \Lambda .
$$


Пусть $\zeta_{n}-$ нуль $Q_{n}$ такой, что $\zeta_{n} \rightarrow a$ при $n \rightarrow \infty$. Тогда для достаточно больших $n$ имеем: $\zeta_{n} \in U_{r}(a)$ и, следовательно,

$$
\left|\frac{P_{n}\left(\zeta_{n}\right)}{A_{n} \zeta_{n}^{n}}\right| \leqslant C_{7}, \quad n \in \Lambda, \quad n \geqslant n_{0} .
$$

С другой стороны, из (1.5) вытекает, что

$$
A_{n} \zeta_{n}^{n}=-\frac{P_{n}\left(\zeta_{n}\right) Q_{n+1}\left(\zeta_{n}\right)}{\zeta_{n}^{m+1-\lambda_{n}}} .
$$

Подставляя последнее соотношение в (1.11), получаем

$$
\frac{1}{\left|Q_{n+1}\left(\zeta_{n}\right)\right|} \leqslant C_{8}, \quad n \in \Lambda, \quad n \geqslant n_{0} .
$$

Но поскольку $\operatorname{dist}\left(a, \mathscr{P}_{n}\right) \rightarrow 0$, имеем: $Q_{n+1}\left(\zeta_{n}\right) \rightarrow 0$ при $n \rightarrow \infty$. Таким образом, предположение $R<|a|$ приводит к противоречию, следовательно, $R \geqslant|a|$.

2.3. Докажем второе утверждение теоремы: если $|a|<R$, то a - полюс функuи $f$. На самом деле мы докажем здесь несколько более сильньй результат: если $|a|<R$, то а - полюс функции $f$, кратность которого равна $\lambda(a)$, где $\lambda(a)$ - число точек множества $\mathscr{P}_{n}$, стремяшихся к точке $a$ при $n \rightarrow \infty$. Очевидно, что $\lambda(a) \geqslant$ $\mu(a)=\nu$, где $\nu$ - кратность полюса $f$ в точке $a$. Поэтому достаточно показать, что $\lambda(a) \leqslant \nu$.

Фиксируем $\rho,|a|<\rho<R$. Из (1.6) вытекает, что

$$
\varlimsup_{n \rightarrow \infty}\left|A_{n} \rho^{n}\right|^{1 / n}=\frac{\rho}{R}<1 .
$$

Следовательно, $\lim _{n \rightarrow \infty}\left|A_{n} \rho^{n}\right|=0$, поэтому найдется бесконечная последовательность $\Lambda=\Lambda(\rho) \subset \mathbb{N}$ такая, что при $n \in \Lambda$ имеем:

$$
\left|A_{n} \rho^{n}\right|=\max _{k \geqslant n}\left|A_{k} \rho^{k}\right| .
$$

Выберем $r>0$ так, чтобы для $2 r$-окрестности $U_{2 r}(a)$ точки $a$ вьполнялись условия: $U_{2 r}(a)$ компактно принадлежит кругу $|z|<\rho$, функция $f$ голоморфна в $U_{2 r}(a) \backslash\{a\}$. В силу сходимости по емкости последовательности $\left\{f_{n}\right\}$ к $f$ внутри круга $D$ представление

$$
f(z)=f_{n}(z)+\sum_{k=n}^{\infty} \frac{A_{k} z^{k+m+1-\lambda_{k}}}{\left(Q_{k} Q_{k+1}\right)(z)},
$$

где $n \in \mathbb{N}$ - произвольное фиксированное, имеет место для "почти всех" окружностей с центром в точке $a$, лежаших в круге $U_{2 r}(a)$. Проведя рассуждения, аналогичные рассуждениям п. 2.2, получим, что для каждого $n \in \Lambda$ существует окружность $\gamma_{n}$ с центром в точке $a$ радиуса $r_{n}, r<r_{n}<2 r$, такая, что

$$
\left|\left(f-f_{n}\right)(z)\right| \leqslant C_{9}\left|A_{n} z^{n}\right|, \quad z \in \gamma_{n}, \quad n \in \Lambda .
$$


Если функция $f$ голоморфна в точке $a($ т.е. $\nu=\mu(a)=0)$, то из $(1.12)$ получаем

$$
\left|\frac{\left(Q_{n} f-P_{n}\right)(z)}{A_{n} z^{n}}\right| \leqslant C_{10}, \quad z \in U_{r}(a), \quad n \in \Lambda .
$$

Последнее неравенство, так же как неравенство (1.10) в п. 2.2, приводит к противоречию.

Пусть теперь $\nu \geqslant 1, \omega(z)=(z-a)^{\nu}$. Тогда из (1.12) получаем

$$
\left|\frac{\left(Q_{n} F-\omega P_{n}\right)(z)}{A_{n} z^{n}}\right| \leqslant C_{11}, \quad z \in U_{r}(a), \quad n \in \Lambda,
$$

где функция $F(z)=\omega(z) f(z)$ голоморфна в $U_{r}(a)$ и $F(a) \neq 0$. Если $\nu \leqslant \lambda(a)-1$, то из сходимости по емкости последовательности $\left\{f_{n}\right\}$ к функции $f$ внутри $D$ вытекает, что полином $P_{n}(z)$ имеет нуль $\eta_{n}$, стремяшийся к точке $a$ при $n \rightarrow \infty$. Поэтому для всех достаточно больших $n \in \Lambda$ из оценки (1.13) получаем

$$
\left|\frac{Q_{n}\left(\eta_{n}\right) F\left(\eta_{n}\right)}{A_{n} \eta_{n}^{n}}\right| \leqslant C_{11},
$$

откуда, с учетом неравенства $F(a) \neq 0$ и соотношения

$$
A_{n} \eta_{n}^{n}=\frac{P_{n+1}\left(\eta_{n}\right) Q_{n}\left(\eta_{n}\right)}{\eta_{n}^{m+1-\lambda_{n}}}
$$

вытекает, что

$$
\frac{1}{\left|P_{n+1}\left(\eta_{n}\right)\right|} \leqslant C_{12}, \quad n \in \Lambda, \quad n \geqslant n_{0} .
$$

В силу сходимости по емкости последовательности $\left\{f_{n}\right\}$ к $f$ и оценки (1.4) имеем:

$$
\left(Q_{n+1} F-\omega P_{n+1}\right)(z) \rightarrow 0 \text { равномерно внутри } U_{r}(a),
$$

$n \rightarrow \infty, n \in \Lambda$. Отсюда вытекает существование такой последовательности $\Lambda_{1} \subset \Lambda$, что $P_{n+1}(z) \rightarrow F_{1}(z)$ равномерно внутри $U_{r}(a)$ при $n \rightarrow \infty, n \in \Lambda_{1}$, где $F_{1}$ голоморфна в точке $a$ и $F_{1}(a)=0$ в силу неравенства $\nu \leqslant \lambda(a)-1$. Таким образом, $P_{n+1}\left(\eta_{n}\right) \rightarrow 0$ при $n \rightarrow \infty, n \in \Lambda_{1} \subset \Lambda$, что противоречит (1.13). Следовательно, $\nu \geqslant \lambda(a)$ и, тем самым, $\lambda(a)=\mu(a)=\nu$. Теорема 1 доказана.

\section{§ 3. Доказательство теоремы 2}

3.1. Заметим сразу, что, как следует из теоремы 1 , точки $a_{1}, \ldots, a_{\mu-1}$ и только они являются полюсами $f$ в круге $|z|<R$. Отсюда уже вытекает, что достаточно рассмотреть случай $R=R_{m}$. Не ограничивая общности, будем считать, что $R_{m}=1$.

Пусть при достаточно больших $n\left(n \geqslant n_{0}\right.$, ниже на номер $n$ будут наложены некоторые дополнительные условия) $f_{n}=[n / m]_{f}=P_{n} / Q_{n}$, где $P_{n}, Q_{n}$ - взаимно простые полиномы и

$$
Q_{n}(z)=\prod_{j=1}^{m}\left(z-\zeta_{n, j}\right)
$$


Имеем:

$$
\begin{aligned}
& \left(Q_{n} f-P_{n}\right)(z)=O\left(z^{n+m+1}\right), \quad z \rightarrow 0, \\
& \left(P_{n+1} Q_{n}-P_{n} Q_{n+1}\right)(z)=A_{n} z^{n+m+1}
\end{aligned}
$$

и

$$
\left(f_{n+1}-f_{n}\right)(z)=\frac{A_{n} z^{n+m+1}}{\left(Q_{n} Q_{n+1}\right)(z)}, \quad n \geqslant n_{0} ;
$$

сходимость последовательности $\left\{f_{n}\right\}$ эквивалентна сходимости ряда

$$
\sum_{n=n_{0}}^{\infty} \frac{A_{n} z^{n+m+1}}{\left(Q_{n} Q_{n+1}\right)(z)}
$$

Последовательность $\left\{f_{n}\right\}$ равномерно сходится к функции $f$ внутри (на компактных подмножествах) области $D_{m}^{\prime}$, получаюшейся из $D_{m}$ удалением полюсов $f$, и имеет место формула

$$
\frac{1}{R_{m}}=\varlimsup_{n \rightarrow \infty}\left|A_{n}\right|^{1 / n}
$$

В соответствии с договоренностью $R_{m}=1$, тем самым,

$$
\varlimsup_{n \rightarrow \infty}\left|A_{n}\right|^{1 / n}=1
$$

3.2. В этом пункте мы сфформулируем лемму, лежащую в основе доказательства теоремы 2 .

Пусть $\left\{\alpha_{n}\right\}$ - произвольная последовательность комплексных чисел такая, что

$$
\varlimsup_{n \rightarrow n}\left|\alpha_{n}\right|^{1 / n}=1
$$

В [54] доказано, что сушествует последовательность положительных чисел $\left\{\alpha_{n}^{*}\right\}$ ( "регуляризация" последовательности $\left\{\alpha_{n}\right\}$ ), удовлетворяюшая следующим условиям:

$1^{\circ} \cdot \alpha_{n}^{*} / \alpha_{n+1}^{*} \rightarrow 1, n \rightarrow \infty$;

$2^{\circ} .\left\{\log \left(\alpha_{n}^{*} / n !\right)\right\}$ - вогнутая (т.е. вьпуклая вверх) последовательность;

$3^{\circ} .\left|\alpha_{n}\right| \leqslant \alpha_{n}^{*}$ для всех $n \in \mathbb{N}$;

$4^{\circ} .\left|\alpha_{n}\right| \geqslant c \alpha_{n}^{*}, c>0$, для $n$, принадлежаших некоторой бесконечной последовательности $\Lambda \subset \mathbb{N}$.

В силу (1.18) для последовательности $\left\{A_{n}\right\}$, определенной соотношением (1.16), найдется последовательность $\left\{A_{n}^{*}\right\}$, удовлетворяюшая условия $1^{\circ}-4^{\circ}$.

ОСНовНАЯ ЛЕмма. Пусть в условиях теоремы 2 функиия $f$ голоморфна в некоторой области $G \supset D_{m}^{\prime}$. Тогда для любого компакта $K \subset G$ имеем:

$$
\max _{z \in K}\left|\frac{\left(Q_{n} f-P_{n}\right)(z)}{A_{n}^{*} z^{n}}\right|=O(1), \quad n \rightarrow \infty
$$


Эта лемма хорошо известна для случая степенного ряда, т.е. при $m=0$ (см., например, [54], где соответствующий результат доказан для рядов Дирихле). В настоящей работе мы обобшаем схему рассуждений [54], при этом вместо разложения функции в степенной ряд используется представление

$$
f(z)=f_{n}(z)+\sum_{k=n}^{\infty} \frac{A_{k} z^{k+m+1}}{\left(Q_{k} Q_{k+1}\right)(z)}
$$

справедливое в условиях теоремы 2 равномерно внутри области $D_{m}^{\prime}$. Ключевым элементом в методе Агмона является использование в доказательстве преобразования Бореля функции, заданной степенным рядом. Наличие в (1.20) знаменателей, зависящих от переменной $z$, не позволяет автоматически перенести эти рассуждения на интересуюший нас более обший случай. Тем не менее удается обойти эти трудности и, тем самым, обобшить метод Агмона на случай произвольного $m \in \mathbb{N}$. Таким образом, из полученных здесь результатов вытекает, что классическому случаю $c_{n} / c_{n+1} \rightarrow a$ "соответствует" как $m=0$, так и $m=1$.

Отметим, что при некотором дополнительном условии на последовательность $\left\{A_{n}\right\}$ (хотя и без какого-либо предположения о поведении конечных полюсов функций $f_{n}$ в целом) аналогичная лемма доказана в $[27]$. Так же как и в классическом случае (т.е. при $m=0$, см., например, [55; с. 118-121]), это условие существенно упрощает рассуждения.

Используя представление (1.20) и свойства последовательности $\left\{A_{n}^{*}\right\}$, нетрудно установить, что в условиях основной леммы имеет место следуюшая

Лемма 1. Для любого $\delta>0$

$$
\max _{|z| \geqslant e^{\delta}}\left|\frac{P_{n}(z)}{A_{n}^{*} z^{n}}\right|=O(1), \quad n \rightarrow \infty ;
$$

положим $G_{\delta}=G \backslash\left\{e^{-\delta} \leqslant|z| \leqslant e^{\delta}\right\}$, тогда для любого компакта $K \subset G_{\delta}$

$$
\max _{z \in K}\left|\frac{\left(Q_{n} f-P_{n}\right)(z)}{A_{n}^{*} z^{n}}\right|=O(1), \quad n \rightarrow \infty .
$$

Таким образом, так же как и в классическом случае, основная трудность при доказательстве основной леммы состоит в проверке соотношения (1.19) для $z$, близких $\kappa$ единичной окружности.

ЛЕмма 2. Если функиия $f$ голоморфна в точке $z_{0},\left|z_{0}\right|=1$, то найдется $\delta=\delta\left(z_{0}\right)>0$ mакое, ито

$$
\max _{\substack{e^{-\delta} \leqslant|z| \leqslant e^{\delta} \\\left|\arg z-\arg z_{0}\right| \leqslant \delta}}\left|\frac{\left(Q_{n} f-P_{n}\right)(z)}{A_{n}^{*} z^{n}}\right|=O(1), \quad n \rightarrow \infty .
$$

Следующие пп. 3.3-3.5 посвящены доказательству леммы 2.

3.3. В этом пункте мы вьведем некоторые оценки, связанные с экспоненщиальной функцией $e^{z}$, и приведем основные свойства преобразования Бореля, которые понадобятся в дальнейшем. 
Пусть $S_{n}(z)-n$-я частная сумма ряда Тейлора с центром в точке $z=0$ функции $e^{z}$. Непосредственно проверяется, что

$$
\left|\frac{e^{z}-S_{n-1}(z)}{\frac{1}{n !} z^{n}}\right| \leqslant \frac{1}{1-|z / n|}, \quad|z|<n,
$$

и

$$
\left|\frac{S_{n-1}(z)}{\frac{1}{n !} z^{n}}\right| \leqslant \frac{1}{|z / n|-1}, \quad|z|>n
$$

Тем самьг,

$$
\max _{|z| \leqslant n e^{-\delta}}\left|\frac{e^{z}-S_{n-1}(z)}{\frac{1}{n !} z^{n}}\right|=O(1), \quad n \rightarrow \infty
$$

и

$$
\max _{|z| \geqslant n e^{\delta}}\left|\frac{S_{n-1}(z)}{z^{n} / n !}\right|=O(1), \quad n \rightarrow \infty
$$

Кроме того, имеем

$$
c(\delta)\left|\frac{z^{k}}{k !}\right| \leqslant\left|\frac{z^{k+m}}{(k+m) !}\right| \leqslant C(\delta)\left|\frac{z^{k}}{k !}\right|,
$$

где $n e^{-\delta} \leqslant|z| \leqslant n e^{\delta}, n e^{-\delta} \leqslant k \leqslant n e^{\delta}$ и величины $C(\delta)$ и $c(\delta)$ зависят только от $\delta$ и $m$ (неравенства (1.28) понадобятся нам в дальнейшем). На луче $\{z: \arg z=\varphi\}$ имеем: $\left|e^{z}\right|=e^{|z| \cos \varphi}$, поэтому для любого $\varepsilon^{\prime}>0$ найдется $\delta^{\prime}=\delta^{\prime}\left(\varepsilon^{\prime}\right)>0$ такое, что

$$
\max _{\substack{n \leqslant|z| \leqslant n e^{\delta^{\prime}} \\|\arg z| \geqslant \varepsilon^{\prime}}}\left|\frac{e^{z}}{\frac{1}{n !} z^{n}}\right|=o(1), \quad n \rightarrow \infty
$$

(легко проверить, что можно взять $\delta^{\prime}=-\frac{1}{2} \log \cos \varepsilon^{\prime}$, если $\left.0<\varepsilon^{\prime}<\pi / 2\right)$. Из (1.24), (1.25) и (1.29) получаем: для любого $\varepsilon>0$ найдется $\delta=\delta(\varepsilon)$ такое, что $0<\delta<\varepsilon$ $u$

$$
\max _{\substack{|z| \leqslant n e^{6 \delta} \\|\arg z| \geqslant \varepsilon}}\left|\frac{e^{z}-S_{n-1}(z)}{\frac{1}{n !} z^{n}}\right|=O(1), \quad n \rightarrow \infty .
$$

Доказательство леммы 2 основано на использовании преобразования Бореля $B(g ; z)$ голоморфной в начале координат функции $g$ (ниже мы записьваем это преобразование в нестандартном, но зато удобном для нас виде). Пусть

$$
g(z)=\sum_{k=0}^{\infty} g_{k} z^{k}
$$

- степенной ряд с единичным радиусом сходимости, тогда

$$
B(g ; z):=\sum_{k=0}^{\infty} \frac{g_{k}}{k !} z^{k}=\frac{1}{2 \pi i} \int_{\Gamma} g(t) e^{z / t} \frac{d t}{t}
$$

где $Г$ - любой контур, лежаший в области голоморфности функции $g(z),|z|<1$, и охватьваюший точку $z=0$. Из интегрального представления (1.31) легко следует, 
что $B(g ; z)$ является целой функцией экспоненциального типа $\leqslant 1$, а если $g$ голоморфна в точке $z_{0}=e^{i \varphi_{0}}$, то тип функции $B(g ; z)$ в некотором угле, содержашем эту точку $z_{0}$, строго $<1$. Точнее, найдутся числа $h, 0<h<1$, и $\varepsilon>0$ такие, что при $\left|\arg z-\varphi_{0}\right| \leqslant \varepsilon$

$$
|B(g ; z)| \leqslant C e^{h|z|}, \quad z \rightarrow \infty
$$

где величина $C$ не зависит от $z$.

Функции $B(g ; \cdot)$ и $g$ назьваются, соответственно, верхней и нижней функциями, ассоциированными по Борелю.

Хорошо известно (см., например, [18]), что $g$ восстанавливается по функции $B(g ; \cdot)$ в окрестности своих точек голоморфности, лежаших на единичной окружности, по формуле:

$$
g\left(r e^{i \varphi}\right)=\frac{1}{r} \int_{0}^{\infty} B\left(g ; \rho e^{i \varphi}\right) e^{-\rho / r} d \rho
$$

При этом из (1.32) легко вытекает, что в окрестности точки $z_{0}=e^{i \varphi_{0}}$, в которой $g$ голоморфна, формула (1.33) справедлива при всех $r \in(0,1 / h)$, где $1 / h>1$, и $\left|\varphi-\varphi_{0}\right| \leqslant \varepsilon$ (отсюда, кстати, с учетом свойств функции $g$ легко следует, что тип функции $B(g ; \cdot)$ в точности равен 1 ; формула (1.33) фактически реализует метод Бореля эффективного аналитического продолжения степенного ряда за пределы круга сходимости).

3.4. Пусть сначала $z_{0} \neq a_{j}$ при $j=\mu, \mu+1, \ldots, m$. Без ограничения общности можем считать $z_{0}=1$. Положим

$$
\omega(z)=\prod_{j=1}^{\mu-1}\left(z-a_{j}\right)
$$

тогда $\operatorname{deg} \omega=\mu-1<m$ и функщия $f \omega$ голоморфна в $D_{m}$ и в точке $z=1$.

В силу условий теоремы 2 коэффициенты полиномов $Q_{n}(z)=z^{m}+\cdots$ ограничены при $n \rightarrow \infty$, поэтому из (1.32) (с заменой функции $g$ на функции $z^{j} \cdot f(z) \omega(z), j=$ $0,1, \ldots, m)$ вытекает, что найдутся не зависящие от $n$ числа $h, 0<h<1$, и $\varepsilon^{\prime}>0$ такие, что при $|\arg z| \leqslant \varepsilon^{\prime}$

$$
\left|B\left(Q_{n} f \omega ; z\right)\right| \leqslant C_{1} e^{h|z|}
$$

(здесь и всюду в этой главе через $C_{1}, C_{2}, \ldots$ обозначаются положительные величины, не зависящие от $n$ и $z$, а также от используемых ниже индекса суммирования $k$ и переменной $t$ ).

Пусть $F_{n}(z):=\left(Q_{n} f \omega-P_{n} \omega\right)(z)$; в соответствии с $(1.33)$ и (1.34) имеем

$$
F_{n}\left(r e^{i \varphi}\right)=\frac{1}{r} \int_{0}^{\infty} B\left(F_{n} ; \rho e^{i \varphi}\right) e^{-\rho / r} d \rho,
$$

где $r \in(0,1 / h)$ и $|\varphi| \leqslant \varepsilon^{\prime}$, причем $h$ и $\varepsilon^{\prime}$ не зависят от $n$. Отметим также, что функция

$$
\frac{F_{n}(z)}{z^{n+m+1}}=\frac{\left(Q_{n} f \omega-P_{n} \omega\right)(z)}{z^{n+m+1}}
$$

голоморфна в $D_{m}($ см. $(1.2))$. 
ПРЕДЛОЖЕНИЕ 1.1. Для любого $\delta>0$

$$
\max _{|z| \leqslant n e^{-\delta}}\left|\frac{B\left(F_{n} ; z\right)}{A_{n}^{*} z^{n} / n !}\right|=O(1), \quad n \rightarrow \infty .
$$

ДокАЗАТЕльство. Пусть $\rho=e^{-\delta / 2}, \Gamma_{\rho}=\{z:|z|=\rho\}$. Тогда на основании (1.31) и (1.36) имеем

$$
\begin{aligned}
B\left(F_{n} ; z\right) & =\frac{1}{2 \pi i} \int_{\Gamma_{\rho}} F_{n}(t) e^{z / t} \frac{d t}{t} \\
& =\frac{1}{2 \pi i} \int_{\Gamma_{\rho}}\left(Q_{n} f \omega-P_{n} \omega\right)(t)\left(e^{z / t}-S_{n-1}\left(\frac{z}{t}\right)\right) \frac{d t}{t} \\
& =\frac{A_{n}^{*}}{n !} z^{n} \frac{1}{\pi} \int_{\Gamma_{\rho}} \frac{\left(Q_{n} f \omega-P_{n} \omega\right)(t)}{A_{n}^{*} t^{n}} \cdot \frac{e^{z / t}-S_{n-1}(z / t)}{\frac{1}{n !}(z / t)^{n}} \frac{d t}{t}
\end{aligned}
$$

Так как при $t \in \Gamma_{\rho}$ и $|z| \leqslant n e^{-\delta}$ имеем: $|z / t| \leqslant n e^{-\delta / 2}$, то из (1.38) в силу (1.22) и (1.26) получаем (1.37).

ПРЕДЛОЖЕНИЕ 1.2. Для любого $\delta>0$

$$
\max _{|z| \geqslant n e^{\delta}}\left|\frac{B\left(P_{n} \omega ; z\right)}{A_{n+m}^{*} z^{n+m} /(n+m) !}\right|=O(1), \quad n \rightarrow \infty .
$$

ДокАЗАТЕльствО. Пусть $\rho=e^{\delta / 2}$, тогда

$$
\begin{aligned}
B\left(P_{n} \omega ; z\right)= & \frac{1}{2 \pi i} \int_{\Gamma_{\rho}}\left(P_{n} \omega\right)(t) e^{z / t} \frac{d t}{t} \\
= & \frac{1}{2 \pi i} \int_{\Gamma_{\rho}}\left(P_{n} \omega\right)(t) S_{n+m-1}\left(\frac{z}{t}\right) \frac{d t}{t} \\
& +\frac{1}{2 \pi i} \int_{\Gamma_{\rho}}\left(P_{n} \omega\right)(t)\left(e^{z / t}-S_{n+m-1}\left(\frac{z}{t}\right)\right) \frac{d t}{t} .
\end{aligned}
$$

Последний интеграл в этом соотношении равен нулю, поскольку $\operatorname{deg} P_{n} \omega \leqslant n+m-1$. Следовательно,

$$
B\left(P_{n} \omega ; z\right)=\frac{A_{n+m}^{*} z^{n+m}}{(n+m) !} \frac{1}{2 \pi i} \int_{\Gamma_{\rho}} \frac{\left(P_{n} \omega\right)(t)}{A_{n}^{*} t^{n}} \cdot \frac{A_{n}^{*}}{A_{n+m}^{*} t^{m}} \cdot \frac{S_{n+m-1}(z / t)}{\frac{1}{(n+m) !}(z / t)^{n+m}} \frac{d t}{t}
$$

Так как $|t|=e^{\delta / 2}$, то при $|z| \geqslant n e^{\delta}$ имеем: $|z / t| \geqslant n e^{\delta / 2} \geqslant(n+m) e^{\delta / 4}$ при достаточно больших $n$. Поэтому из последнего представления в силу $(1.21),(1.27)$ и свойства $1^{\circ}$ последовательности $\left\{A_{n}^{*}\right\}$ вытекает (1.39). 
ПРЕДЛОЖЕНИЕ 1.3. Если функиия $f$ голоморфна в точке $z_{0}=1$, то найдется $\delta>0$ maкое, что

$$
\max _{\substack{n e^{-\delta \leqslant|z| \leqslant n} \\|\arg z| \leqslant \delta}}\left|\frac{B\left(F_{n} ; z\right)}{A_{n}^{*} z^{n} / n !}\right|=O(1), \quad n \rightarrow \infty .
$$

ДокАЗАТЕльство. Покажем, что существуют числа $\delta>0$ и $\varepsilon>0$ такие, что при $n e^{-\delta} \leqslant|z| \leqslant n e^{\delta}$ и $|\arg z| \leqslant \varepsilon$

$$
\left|B\left(F_{n} ; z\right)\right| \leqslant C_{2} \sum_{k=n}^{\infty} \frac{A_{k}^{*}}{k !}|z|^{k}
$$

и

$$
\left|B\left(F_{n} ; z\right)\right| \leqslant C_{3} \sum_{k=n_{0}}^{n-1} \frac{A_{k}^{*}}{k !}|z|^{k}
$$

при достаточно больших $n$. Пусть $\varepsilon$ удовлетворяет следующим условиям: $0<\varepsilon<$ $\varepsilon^{\prime} / 2$, функция $f$ голоморфна на множестве

$$
\Delta_{\varepsilon}=\left\{z: e^{-2 \varepsilon} \leqslant|z| \leqslant e^{2 \varepsilon},|\arg z| \leqslant 2 \varepsilon\right\}
$$

и все точки $a_{1}, a_{2}, \ldots, a_{m}$ лежат вне $\Delta_{\varepsilon}$.

Выберем теперь $\delta=\delta(\varepsilon, h) \in(0, \varepsilon)$ так, чтобы имело место (1.30) и вьполнялись соотношения

$$
x^{x} e^{e^{2 \delta} \cos \varepsilon+2 \delta x-x}<1
$$

для всех $x \in\left[e^{-3 \delta}, 1\right]$,

$$
e^{h e^{\delta}-1+\delta}<1
$$

(по поводу величины $h$ см. (1.34)),

$$
e^{h-e^{-\delta}}<1
$$

и $e^{-\delta}>\left|a_{\mu-1}\right|$ (напомним, что $0<\left|a_{1}\right| \leqslant \cdots \leqslant\left|a_{\mu-1}\right|<\left|a_{\mu}\right|=\cdots=\left|a_{m}\right|=R$ ), смысл этих требований станет ясен позднее; отметим, что $\left(1.43_{3}\right)$ следует из $\left(1.43_{2}\right)$.

Выберем, наконеи, $n_{0}=n_{0}(\varepsilon, \delta)$ так, чтобы все конечные полюсы функций $f_{n}$ при $n \geqslant n_{0}$ принадлежсали множеству $\left\{z:|z|<e^{\delta / 2}\right\} \backslash \Delta_{\varepsilon}$, а окружность $|z|=e^{-\delta}$ не содержала бы полюсов функций $f_{n}$.

Докажем сначала соотношение (1.41). Пусть $\rho=e^{-\delta}<1$, тогда в соответствии с представлением (1.20)

$$
\begin{aligned}
B\left(F_{n} ; z\right)= & \frac{1}{2 \pi i} \int_{\Gamma_{\rho}} F_{n}(t) e^{z / t} \frac{d t}{t} \\
= & \sum_{k=n}^{\infty} \frac{1}{2 \pi i} \int_{\Gamma_{\rho}} \frac{A_{k} t^{k+m+1}}{\left(Q_{k} Q_{k+1}\right)(t)}\left(Q_{n} \omega\right)(t)\left(e^{z / t}-S_{k-1}\left(\frac{z}{t}\right)\right) \frac{d t}{t} \\
& \quad+\sum_{k=n}^{\infty} \frac{1}{2 \pi i} \int_{\Gamma_{\rho}} \frac{A_{k} t^{k+m+1}}{\left(Q_{k} Q_{k+1}\right)(t)}\left(Q_{n} \omega\right)(t) S_{k-1}\left(\frac{z}{t}\right) \frac{d t}{t} \\
= & \Sigma_{1}(z)+\Sigma_{2}(z),
\end{aligned}
$$


где через $\Sigma_{1}(z)$ и $\Sigma_{2}(z)$ обозначены соответственно первая и вторая суммы в предшествующем выражении.

Пусть теперь кривая $\gamma$ есть граница множества $\left\{z:|z| \leqslant e^{-\delta}\right\} \cup \Delta_{\varepsilon}$. В силу выбора $\varepsilon$ и $n \geqslant n_{0}$ имеем

$$
\begin{aligned}
\Sigma_{1}(z) & =\sum_{k=n}^{\infty} \frac{1}{2 \pi i} \int_{\gamma} \frac{A_{k} t^{k+m+1}}{\left(Q_{k} Q_{k+1}\right)(t)}\left(Q_{n} \omega\right)(t)\left(e^{z / t}-S_{k-1}\left(\frac{z}{t}\right)\right) \frac{d t}{t} \\
& =\sum_{k=n}^{\infty} \frac{A_{k}}{k !} z^{k} \frac{1}{2 \pi i} \int_{\gamma} \frac{t^{m+1}}{\left(Q_{k} Q_{k+1}\right)(t)}\left(Q_{n} \omega\right)(t) \frac{e^{z / t}-S_{k-1}(z / t)}{\frac{1}{k !}(z / t)^{k}} \frac{d t}{t} .
\end{aligned}
$$

Если $|z| \leqslant n e^{\delta},|\arg z| \leqslant \varepsilon$, то при $t \in \gamma$ имеем: либо $|z / t| \leqslant n e^{2 \delta}$ и $|\arg (z / t)| \geqslant \varepsilon$, либо $|z / t| \leqslant n e^{-\delta}$. Поэтому, в силу (1.26) и (1.30), получаем

$$
\left|\frac{e^{z / t}-S_{k-1}(z / t)}{\frac{1}{k !}(z / t)^{k}}\right| \leqslant C_{4}
$$

равномерно по $z \in K_{n}(\varepsilon, \delta), t \in \gamma$ и $k \geqslant n$, где $K_{n}(\varepsilon, \delta)=\left\{z:|z| \leqslant n e^{\delta},|\arg z| \leqslant \varepsilon\right\}$. Следовательно, из (1.44) вытекает неравенство

$$
\left|\Sigma_{1}(z)\right| \leqslant C_{5} \sum_{k=n}^{\infty} \frac{A_{k}^{*}}{k !}|z|^{k}, \quad z \in K_{n}(\varepsilon, \delta) .
$$

Займемся теперь величиной $\Sigma_{2}(z)$ :

$$
\Sigma_{2}(z)=\sum_{k=n}^{\infty} \frac{1}{2 \pi i} \int_{\Gamma_{\rho}}\left(\left(f_{k+1}-f\right)+\left(f-f_{k}\right)\right)(t)\left(Q_{n} \omega\right)(t) S_{k-1}\left(\frac{z}{t}\right) \frac{d t}{t}
$$

Tak как

$$
\begin{aligned}
\sum_{k=n}^{N-1} & \frac{1}{2 \pi i} \int_{\Gamma_{\rho}}\left(\left(f_{k+1}-f\right)+\left(f-f_{k}\right)\right)(t)\left(Q_{n} \omega\right)(t) S_{k-1}\left(\frac{z}{t}\right) \frac{d t}{t} \\
= & \frac{1}{2 \pi i} \int_{\Gamma_{\rho}}\left(Q_{n} f \omega-P_{n} \omega\right)(t) S_{n-1}\left(\frac{z}{t}\right) \frac{d t}{t} \\
& -\frac{1}{2 \pi i} \int_{\Gamma_{\rho}}\left(f-f_{N}\right)(t)\left(Q_{n} \omega\right)(t) S_{N-2}\left(\frac{z}{t}\right) \frac{d t}{t} \\
& +\sum_{k=n}^{N-1} \frac{1}{2 \pi i} \int_{\Gamma_{\rho}}\left(f-f_{k+1}\right)(t)\left(Q_{n} \omega\right)(t)\left(S_{k}\left(\frac{z}{t}\right)-S_{k-1}\left(\frac{z}{t}\right)\right) \frac{d t}{t}
\end{aligned}
$$

и равномерно по $t \in \Gamma_{\rho}$ и $|z| \leqslant R$ (здесь $R \in(0, \infty)$ - любое действительное число) имеем

$$
\left(f-f_{N}\right)(t) \rightarrow 0, \quad S_{N-2}\left(\frac{z}{t}\right) \rightarrow e^{z / t} \text { при } \quad N \rightarrow \infty
$$


то из (1.46) с учетом голоморфности функции (1.36) получаем

$$
\Sigma_{2}(z)=\sum_{k=n}^{\infty} \frac{1}{2 \pi i} \int_{\Gamma_{\rho}}\left(f-f_{k+1}\right)(t)\left(Q_{n} \omega\right)(t) \frac{1}{k !}\left(\frac{z}{t}\right)^{k} \frac{d t}{t}
$$

Откуда в силу леммы 1 имеем

$$
\left|\Sigma_{2}(z)\right| \leqslant C_{6} \sum_{k=n}^{\infty} \frac{A_{k}^{*}}{k !}|z|^{k}, \quad z \in K_{n}(\varepsilon, \delta) .
$$

Из (1.45) и (1.47) следует (1.41) при $z \in K_{n}(\varepsilon, \delta)$.

Докажем теперь соотношение (1.42). Пусть $R=e^{\delta}>1, \rho=e^{-\delta}<1$. Тогда в силу выбора $n_{0}$ имеем: все конечные полюсы функций $f_{n}, n \geqslant n_{0}$, лежат внутри $\Gamma_{R}$. Пользуясь этим фактом и равенством

$$
\left(P_{n} \omega\right)(z)=\left(f_{n_{0}} Q_{n} \omega\right)(z)+\sum_{k=n_{0}}^{n-1} \frac{A_{k} z^{k+m+1}}{\left(Q_{k} Q_{k+1}\right)(z)}\left(Q_{n} \omega\right)(z)
$$

получаем

$$
\begin{aligned}
B\left(F_{n} ; z\right)= & B\left(Q_{n} f \omega ; z\right)-B\left(P_{n} \omega ; z\right) \\
= & B\left(Q_{n} f \omega ; z\right)-\frac{1}{2 \pi i} \int_{\Gamma_{R}}\left(f_{n_{0}} Q_{n} \omega\right)(t) S_{n_{0}+m}\left(\frac{z}{t}\right) \frac{d t}{t} \\
& -\sum_{k=n_{0}}^{n-1} \frac{1}{2 \pi i} \int_{\Gamma_{R}} \frac{A_{k} t^{k+m+1}}{\left(Q_{k} Q_{k+1}\right)(t)}\left(Q_{n} \omega\right)(t) S_{k+m}\left(\frac{z}{t}\right) \frac{d t}{t} \\
= & B\left(Q_{n} f \omega ; z\right)-I(z)-\Sigma(z)
\end{aligned}
$$

(интегралы, содержашие члены вида $e^{z / t}-S_{k+m}(z / t)$, обращаются в нуль). Пусть $n>n_{0}^{\prime} \geqslant e^{3 \delta}\left(n_{0}+m\right)$, тогда при $|z| \geqslant n e^{-\delta}$ и $t \in \Gamma_{R}$ имеем: $|z / t| \geqslant n e^{-2 \delta} \geqslant$ $e^{\delta}\left(n_{0}+m\right)$. Следовательно,

$$
\left|S_{n_{0}+m}\left(\frac{z}{t}\right)\right| \leqslant C_{7} \frac{1}{\left(n_{0}+m\right) !}\left|\frac{z}{t}\right|^{n_{0}+m} .
$$

Таким образом,

$$
|I(z)| \leqslant C_{8} \frac{A_{n_{0}+m}^{*}}{\left(n_{0}+m\right) !}|z|^{n_{0}+m} \text { при }|z| \geqslant n e^{-\delta} .
$$

Оценим теперь $\Sigma(z)$. Пусть

$$
\begin{aligned}
L=\left\{t:|t|=e^{\delta},|\arg t| \geqslant 2 \varepsilon\right\} & \cup\left\{t:|t|=e^{-\delta},|\arg t| \geqslant 2 \varepsilon\right\} \\
\cup & \left\{t: e^{-\delta} \leqslant|t| \leqslant e^{\delta},|\arg t|=2 \varepsilon\right\} .
\end{aligned}
$$


Тогда

$$
\begin{aligned}
\Sigma(z)= & \sum_{k=n_{0}}^{n-1} \frac{1}{2 \pi i} \int_{\Gamma_{R}} \frac{A_{k} z^{k+m+1}}{\left(Q_{k} Q_{k+1}\right)(t)}\left(Q_{n} \omega\right)(t) S_{k+m}\left(\frac{z}{t}\right) \frac{d t}{t} \\
& +\sum_{k=n_{0}}^{n-1} \frac{1}{2 \pi i} \int_{L} \frac{A_{k} t^{k+m+1}}{\left(Q_{k} Q_{k+1}\right)(t)}\left(Q_{n} \omega\right)(t) S_{k+m}\left(\frac{z}{t}\right) \frac{d t}{t} \\
= & \Sigma_{1}(z)+\Sigma_{2}(z) .
\end{aligned}
$$

С учетом голоморфности функции (1.36) имеем

$$
\begin{aligned}
\Sigma_{1}(z)= & \sum_{k=n_{0}}^{n-1} \frac{1}{2 \pi i} \int_{\Gamma_{\rho}}\left(\left(f_{k+1}-f\right)+\left(f-f_{k}\right)\right)(t)\left(Q_{n} \omega\right)(t) S_{k+m}\left(\frac{z}{t}\right) \frac{d t}{t} \\
= & \frac{1}{2 \pi i} \int_{\Gamma_{\rho}}\left(f-f_{n_{0}}\right)(t)\left(Q_{n} \omega\right)(t) S_{n_{0}+m}\left(\frac{z}{t}\right) \frac{d t}{t} \\
& +\sum_{k=n_{0}}^{n-2} \frac{1}{2 \pi i} \int_{\Gamma_{\rho}}\left(f-f_{k+1}\right)(t)\left(Q_{n} \omega\right)(t) \frac{1}{(k+m) !}\left(\frac{z}{t}\right)^{k+m+1} \frac{d t}{t} .
\end{aligned}
$$

Пусть $n \geqslant n_{0}^{\prime}$. Тогда в силу (1.49), леммы 1 и (1.28) получаем из (1.51)

$$
\left|\Sigma_{1}(z)\right| \leqslant C_{9} \sum_{k=n_{0}}^{n-1} \frac{A_{k}^{*}}{k !}|z|^{k}, \quad n e^{-\delta} \leqslant|z| \leqslant n e^{\delta} .
$$

Оценим теперь $\Sigma_{2}(z)$. Пусть $\lambda=e^{-3 \delta}<1$. Запишем $\Sigma_{2}(z)$ в следуюшем виде $([x]$ означает целую часть числа $x)$ :

$$
\begin{aligned}
\Sigma_{2}(z)= & \sum_{k=n_{0}}^{[\lambda n]-m} \frac{1}{2 \pi i} \int_{L} \frac{A_{k} t^{k+m+1}}{\left(Q_{k} Q_{k+1}\right)(t)}\left(Q_{n} \omega\right)(t) S_{k+m}\left(\frac{z}{t}\right) \frac{d t}{t} \\
& -\sum_{k=[\lambda n]-m+1}^{n-1} \frac{1}{2 \pi i} \int_{L} \frac{A_{k} t^{k+m+1}}{\left(Q_{k} Q_{k+1}\right)(t)}\left(Q_{n} \omega\right)(t)\left(e^{z / t}-S_{k+m}\left(\frac{z}{t}\right)\right) \frac{d t}{t} \\
& +\sum_{k=[\lambda n]-m+1}^{n-1} \frac{1}{2 \pi i} \int_{L} \frac{A_{k} t^{k+m+1}}{\left(Q_{k} Q_{k+1}\right)(t)}\left(Q_{n} \omega\right)(t) e^{z / t} \frac{d t}{t} \\
= & \Sigma_{2,1}(z)-\Sigma_{2,2}(z)+\Sigma_{2,3}(z) .
\end{aligned}
$$

Пусть $n e^{-\delta} \leqslant|z| \leqslant n e^{\delta}$. При $t \in L$ имеем: $|t| \leqslant e^{\delta}$, поэтому $|z / t| \geqslant n e^{-2 \delta}$. Если $n_{0} \leqslant k \leqslant[\lambda n]-m$, то

$$
|z / t| \geqslant n e^{-2 \delta}=\lambda n e^{\delta} \geqslant(k+m) e^{\delta},
$$

следовательно, при указанных $z, t$ и $k$ в силу (1.27)

$$
\left|S_{k+m}\left(\frac{z}{t}\right)\right| \leqslant \frac{C_{10}}{(k+m) !}\left|\frac{z}{t}\right|^{k+m}
$$


и

$$
\left|\Sigma_{2,1}(z)\right| \leqslant C_{11} \sum_{k=n_{0}}^{[\lambda n]-m} \frac{\left|A_{k}\right|}{k !}|z|^{k}, \quad n e^{-\delta} \leqslant|z| \leqslant n e^{\delta} .
$$

Пусть теперь $[\lambda n]-m+1 \leqslant k \leqslant n-1$, тогда при $|z| \leqslant n e^{\delta},|\arg z| \leqslant \varepsilon$ и $t \in L$ имеем: $|z / t| \leqslant n e^{2 \delta}<(k+m) e^{5 \delta},|\arg (z / t)| \geqslant \varepsilon$. Тем самым в силу (1.30)

$$
\left|e^{z / t}-S_{k+m}\left(\frac{z}{t}\right)\right| \leqslant \frac{C_{12}}{(k+m) !}\left|\frac{z}{t}\right|^{k+m} .
$$

Поэтому с учетом (1.28) получаем

$$
\left|\Sigma_{2,2}(z)\right| \leqslant C_{13} \sum_{k=[\lambda n]-m+1}^{n-1} \frac{\left|A_{k}\right|}{k !}|z|^{k}, \quad n e^{-\delta} \leqslant|z| \leqslant n e^{\delta}, \quad|\arg z| \leqslant \varepsilon .
$$

При $t \in L,|\arg z| \leqslant \varepsilon$ имеем: $\operatorname{Re}(z / t) \leqslant|z / t| \cos \varepsilon \leqslant|z| e^{\delta} \cos \varepsilon$, следовательно,

$$
\left|e^{z / t}\right| \leqslant e^{|z| e^{\delta} \cos \varepsilon}
$$

Поэтому равномерно по $z \in K_{n}(\varepsilon, \delta)$ и $t \in L$

$$
\varlimsup_{n \rightarrow \infty}\left|e^{z / t}\right|^{1 / n} \leqslant e^{e^{2 \delta} \cos \varepsilon}
$$

Далее, при $[\lambda n]-m+1 \leqslant k \leqslant n-1,|z| \geqslant n e^{-\delta}$ и $t \in L$

$$
\left|\frac{k !}{(z / t)^{k}}\right| \leqslant C \frac{k^{k+1} e^{-k}}{n^{k} e^{-2 k \delta}}=C k\left(\frac{k}{n}\right)^{k} e^{-k+2 k \delta},
$$

поэтому

$$
\varlimsup_{n \rightarrow \infty} \max \left|\frac{k !}{(z / t)^{k}}\right|^{1 / n} \leqslant \max _{e^{-3 \delta} \leqslant x \leqslant 1} x^{x} e^{-x+2 x \delta}=a^{a} e^{-a+2 a \delta}
$$

(максимум в левой части (1.56) берется по вьшеуказанным $k, z$ и $t$ ).

Из $(1.55),(1.56)$ и $\left(1.43_{1}\right)$ получаем, что равномерно по $z \in E_{n}(\varepsilon, \delta)=\{z:|\arg z| \leqslant \varepsilon$, $\left.n e^{-\delta} \leqslant|z| \leqslant n e^{\delta}\right\}, t \in L$ и $[\lambda n]-m+1 \leqslant k \leqslant n-1$ имеем

$$
\varlimsup_{n \rightarrow \infty}\left|\frac{e^{z / t}}{\frac{1}{k !}(z / t)^{k}}\right|^{1 / n} \leqslant a^{a} e^{e^{2 \delta} \cos \varepsilon-a+2 a \delta}<1 .
$$

Следовательно,

$$
\left|\Sigma_{2,3}(z)\right| \leqslant C_{14} \sum_{k=[\lambda n]-m+1}^{n-1} \frac{\left|A_{k}\right|}{k !}|z|^{k}, \quad z \in E_{n}(\varepsilon, \delta) .
$$


Из (1.52)-(1.54) и (1.57) получаем

$$
|\Sigma(z)| \leqslant C_{15} \sum_{k=n_{0}}^{n-1} \frac{A_{k}^{*}}{k !}|z|^{k}, \quad z \in E_{n}(\varepsilon, \delta) .
$$

Далее, равномерно по $z \in E_{n}(\varepsilon, \delta)$ имеем с учетом (1.34) и $\left(1.43_{2}\right)$ :

$$
\varlimsup_{n \rightarrow \infty}\left|\frac{B\left(Q_{n} f \omega ; z\right)}{\frac{A_{n-1}^{*}}{(n-1) !} z^{n-1}}\right|^{1 / n} \leqslant e^{h e^{\delta}+\delta-1}<1
$$

поэтому

$$
\left|B\left(Q_{n} f \omega ; z\right)\right| \leqslant C_{16} \frac{A_{n-1}^{*}}{(n-1) !}|z|^{n-1}, \quad z \in E_{n}(\varepsilon, \delta) .
$$

Из (1.48), (1.50), (1.58) и (1.59) вытекает (1.42).

Покажем теперь, как из (1.41) и (1.42) следует (1.40). Положим

$$
R_{n}=\frac{A^{*}}{(n-1) !} / \frac{A_{n}^{*}}{n !}=n \frac{A_{n-1}^{*}}{A_{n}^{*}}=n e^{\theta_{n}}
$$

где $\theta_{n} \rightarrow 0$ при $n \rightarrow \infty$. В таком случае из $(1.41)$ и (1.42) соответственно получаем

$$
\begin{aligned}
& \left|B\left(F_{n} ; z\right)\right| \leqslant C_{2} \sum_{k=n}^{\infty} \frac{A_{k}^{*}}{k !}|z|^{k}, \quad n e^{-\delta} \leqslant|z| \leqslant R_{n}, \quad|\arg z| \leqslant \varepsilon, \\
& \left|B\left(F_{n} ; z\right)\right| \leqslant C_{3} \sum_{k=n_{0}}^{n-1} \frac{A_{k}^{*}}{k !}|z|^{k}, \quad R_{n} \leqslant|z| \leqslant n e^{\delta}, \quad|\arg z| \leqslant \varepsilon .
\end{aligned}
$$

Отсюда с учетом свойства вогнутости последовательности $\left\{\log \left(A_{n}^{*} / n !\right)\right\}$ вытекает, что

$$
\left|\frac{B\left(F_{n} ; z\right)}{A_{n}^{*} z^{n} / n !}\right| \leqslant \frac{C_{17}}{1-\left|z / R_{n}\right|}, \quad n e^{-\delta} \leqslant|z|<R_{n}, \quad|\arg z| \leqslant \varepsilon,
$$

и

$$
\left|\frac{B\left(F_{n} ; z\right)}{A_{n}^{*} z^{n} / n !}\right| \leqslant \frac{C_{18}}{\left|z / R_{n}\right|-1}, \quad R_{n}<|z| \leqslant n e^{\delta}, \quad|\arg z| \leqslant \varepsilon .
$$

Из (1.60) и (1.61) стандартным методом (см., например, [55; с. 118-121], а также [54] и [27]) получаем

$$
\left|\frac{B\left(F_{n} ; z\right)}{\left(A_{n}^{*} / n !\right) z^{n}}\right| \leqslant C_{19}, \quad n e^{-\delta} \leqslant|z| \leqslant n e^{\delta}, \quad|\arg z| \leqslant \frac{\varepsilon}{2},
$$

откуда следует (1.40). Предложение 1.3 доказано. 
3.5. Приступим непосредственно к доказательству леммы 2. Из (1.37) и (1.40) вытекает, что

$$
\max _{\substack{|z| \leqslant n e^{\delta} \\|\arg z| \leqslant \delta}}\left|\frac{B\left(F_{n} ; z\right)}{\frac{A_{n}^{*}}{n !} z^{n}}\right|=O(1), \quad n \rightarrow \infty .
$$

Запишем соотношение (1.35) в виде

$$
\begin{aligned}
F_{n}\left(r e^{i \varphi}\right)= & \frac{1}{r} \int_{0}^{n e^{\delta}} B\left(F_{n} ; \rho e^{i \varphi}\right) e^{-\rho / r} d \rho \\
& +\frac{1}{r} \int_{n e^{\delta}}^{\infty} B\left(Q_{n} f \omega ; \rho e^{i \varphi}\right) e^{-\rho / r} d \rho \\
& -\frac{1}{r} \int_{n e^{\delta}}^{\infty} B\left(P_{n} \omega ; \rho e^{i \varphi}\right) e^{-\rho / r} d \rho \\
= & I_{n, 1}(r, \varphi)+I_{n, 2}(r, \varphi)-I_{n, 3}(r, \varphi),
\end{aligned}
$$

где $e^{-\delta} \leqslant r \leqslant e^{\delta},|\varphi| \leqslant \delta$. На основании (1.62) получаем

$$
\begin{aligned}
\left|I_{n, 1}(\rho, \varphi)\right| & \leqslant C_{20} \frac{A_{n}^{*}}{n !} \int_{0}^{n e^{\delta}} \rho^{n} e^{-\rho / r} \frac{d \rho}{r} \\
& \leqslant C_{20} \frac{A_{n}^{*}}{n !} r^{n} \int_{0}^{\infty} x^{n} e^{-x} d x=C_{20} A_{n}^{*} r^{n} .
\end{aligned}
$$

Далее, в силу $(1.34)$ и $\left(1.43_{3}\right)$ имеем

$$
\begin{aligned}
\left|I_{n, 2}(r, \varphi)\right| & \leqslant C_{21} \int_{n e^{\delta}}^{\infty} e^{(h-1 / r) \rho} d \rho \\
& \leqslant C_{21} \int_{n e^{\delta}}^{\infty} e^{\left(h-e^{-\delta}\right) \rho} d \rho=C_{22} e^{\left(h-e^{-\delta}\right) n e^{\delta}},
\end{aligned}
$$

следовательно, с учетом $\left(1.43_{2}\right)$,

$$
\varlimsup_{n \rightarrow \infty}\left|I_{n, 2}(r, \varphi)\right|^{1 / n} \leqslant e^{\left(h-e^{-\delta}\right) e^{\delta}}=e^{e^{\delta} h-1}<e^{-\delta} .
$$

Так как при $e^{-\delta} \leqslant r \leqslant e^{\delta}$

$$
\lim _{n \rightarrow \infty}\left(A_{n}^{*} r^{n}\right)^{1 / n} \geqslant e^{-\delta}
$$

то

$$
\left|I_{n, 2}(r, \varphi)\right| \leqslant C_{23} A_{n}^{*} r^{n}, \quad e^{-\delta} \leqslant r \leqslant e^{\delta}, \quad|\varphi| \leqslant \delta .
$$

Оценим теперь $I_{n, 3}(\rho, \varphi)$. Учитьвая $(1.35)$ и свойство $1^{\circ}$ последовательности $\left\{A_{n}^{*}\right\}$, получаем

$$
\begin{aligned}
\left|I_{n, 3}(r, \varphi)\right| & \leqslant C_{24} \frac{A_{n+m}^{*}}{(n+m) !} \int_{n e^{\delta}}^{\infty} \rho^{n+m} e^{-\rho / r} \frac{d \rho}{r} \\
& \leqslant C_{24} \frac{A_{n+m}^{*}}{(n+m) !} r^{n+m} \int_{0}^{\infty} x^{n+m} e^{-x} d x \\
& \leqslant C_{25} A_{n}^{*} r^{n}, \quad e^{-\delta} \leqslant r \leqslant e^{\delta} .
\end{aligned}
$$


Из (1.63)-(1.65) вытекает, что

$$
\left|F_{n}\left(r e^{i \varphi}\right)\right| \leqslant C_{26} A_{n}^{*} r^{n}, \quad e^{-\delta} \leqslant r \leqslant e^{\delta}, \quad|\varphi| \leqslant \delta .
$$

Итак, лемма 2 доказана при условии $z_{0} \neq a_{j}, j=\mu, \mu+1, \ldots, m$. В общем случае соотношение (1.23) получаем для соседних с $z_{0}$ точек. Вместе с леммой 1 это дает равномерную (по $z$ и $n$ ) ограниченность семейства голоморфных функций $\left\{F_{n}(z) / A_{n}^{*} z^{n}\right\}$ на границе некоторого множества $K_{\delta}=\left\{z: e^{-\delta} \leqslant|z| \leqslant e^{\delta},\left|\arg z-\arg z_{0}\right| \leqslant \delta\right\}$. По принципу максимума модуля для голоморфных функций получаем равномерную ограниченность этого семейства и на всем множестве $K_{\delta}$, т.е. в окрестности точки $z_{0}$. Таким образом, лемма 2 и, тем самым, основная лемма доказаны.

3.6. ДОКАЗАТЕЛЬСТВо ТЕОРемЫ 2 . Пусть $z_{0}=a_{j}$ при некотором $j \in\{\mu, \mu+1$, $\ldots, m\},|a|=1$. Предположим, что $z_{0}$ является точкой голоморфности функции $f$. Тогда по основной лемме соотношение (1.19) имеет место в некоторой окрестности $U$ точки $z_{0}$. В силу свойства $4^{\circ}$ последовательности $\left\{A_{n}^{*}\right\}$ имеем:

$$
\left|\frac{\left(Q_{n} f-P_{n}\right)(z)}{A_{n} z^{n}}\right|=O(1), \quad n \rightarrow \infty, \quad n \in \Lambda,
$$

равномерно по $z \in U$. Пусть $\xi_{n}-$ нуль $Q_{n}$ такой, что $\xi_{n} \rightarrow z_{0}$ при $n \rightarrow \infty$. Тогда при всех достаточно больших $n$ точка $\xi_{n} \in U$. Следовательно, полагая в (1.66) $z=\xi_{n}$, получаем

$$
\left|\frac{P_{n}\left(\xi_{n}\right)}{A_{n} \xi_{n}^{n}}\right| \leqslant C_{27}, \quad n \rightarrow \infty, \quad n \in \Lambda .
$$

С другой стороны, из (1.16) вытекает, что

$$
A_{n} \xi_{n}^{n}=-\frac{P_{n}\left(\xi_{n}\right) Q_{n+1}\left(\xi_{n}\right)}{\xi_{n}^{m+1}} .
$$

Подставляя последнее соотношение в (1.67), получаем

$$
\left|\frac{1}{Q_{n+1}\left(\xi_{n}\right)}\right| \leqslant C_{28}, \quad n \rightarrow \infty, \quad n \in \Lambda .
$$

Но $Q_{n+1}\left(\xi_{n}\right) \rightarrow 0$, поскольку $\xi_{n} \rightarrow z_{0}=a_{j}$ при $n \rightarrow \infty$. Таким образом, предположение о голоморфности функции $f$ в точке $z_{0}=a_{j}$ привело к противоречию. Следовательно, каждая точка $a_{j}, j=\mu, \ldots, m,-$ особая точка функции $f(z)$ на границе круга $|z|<1$. Теорема 2 доказана.

ЗАмечАниЕ. Теорема 2 допускает некоторое обобщение в виде "локального" варианта, т.е. без предположения, что все точки множества $\mathscr{P}_{n}$ конечных полюсов функции $f_{n}$ имеют пределы при $n \rightarrow \infty$. Точнее, пусть существует точка $a \in \mathbb{C}, a \neq 0$, такая, что

$$
\operatorname{dist}\left(a, \mathscr{P}_{n}\right)=\min _{\xi \in \mathscr{P}{ }_{n}}|a-\xi| \rightarrow 0, \quad n \rightarrow \infty .
$$


Из теоремы 1 вытекает, что в этом случае $R_{m} \geqslant|a|$, и если $|a|<R_{m}$, то $a$ - полюс функции $f$. Приведенные здесь методы доказательства теорем 1 и 2 позволяют получить следующий результат: если в условия теоремь $1|a|=R_{m}$ и найдется окрестность $U$ точки а такая, что

$$
\max _{\xi \in \mathscr{P}_{n} \cap U}|a-\xi| \rightarrow 0, \quad n \rightarrow \infty
$$

то а - особая точка функиии $f(z)$ на граниче круга $|z|<R_{m}$.

Отметим, что в [27] аналогичньй результат получен без ограничения (1.68), но при другом дополнительном условии на поведение последовательности $\left\{A_{n}\right\}$. При таком условии доказательство сушественно упрошается и проводится с использованием известной классической схемы (см. п. 3.2 настоящего параграффа, замечание по поводу основной леммы).

\section{Глава II. \\ Равномерная сходимость диагональных аппроксимаций Паде для некоторых классов аналитических функций}

В этой главе изучается задача о восстановлении главной диагональю Паде дивизора полюсов находящейся в "общем положении” произвольной функции из класса Наттолла, являюшегося естественным обобщением класса гиперэллиптических функций. В частности, для такой функции доказывается соответствующий вариант гипотезы Бейкера-Гамелля-Уиллса о существовании равномерно сходящейся в сферической метрике подпоследовательности диагональных аппроксимаций Паде. Анализ равномерной сходимости главной диагонали Паде в работе опирается на хорошо известньй факт, что для функций из класса Наттолла знаменатели аппроксимаций - полиномы, неэрмитово ортогональные относительного комплексного веса на аналитических дугах, обладаюших определенньм свойством “симметрии”. Сильная асимптотика таких ортогональных полиномов описьвается в работе в терминах решения определенной краевой задачи Римана на соответствующей гиперэллиптической римановой поверхности. В свою очередь, анализ сушествования и нахождение “явного" вида решения этой задачи дается в работе в терминах обладаюшего спешиальными свойствами решения проблемы Якоби обрашения абелевых интегралов. Наконец, используя свойства этого решения задачи Римана, мы вьводим сингулярное интегральное уравнение для полиномов Паде, первьй вариант которого в случае $\mathrm{g}=0$, т.е. при $S=[-1,1]$, был получен Наттоллом. Основные результаты настоящей главы опубликованы в работах автора [28], [29].

\section{$\S$ 1. Постановка задачи и формулировка основных результатов}

1.1. Пусть

$$
f(z)=\sum_{k=0}^{\infty} \frac{c_{k}}{z^{k+1}}
$$

- функция, голоморфная в бесконечно удаленной точке $z=\infty$. Хорошо известно (см. прежде всего классические работы П. Л. Чебьшёва [56] и А. А. Маркова [57]), что если 
все ганкелевы определители, составленные из коэффищиентов $\left\{c_{k}\right\}$, отличны от нуля, то $f$ можно сопоставить чебышёвскую непрерывную дробь

$$
\frac{b_{1}}{z-a_{1}-\frac{b_{2}}{z-a_{2}-\cdot}},
$$

подходящие дроби $P_{n} / Q_{n}$ которой обладают следуюшим замечательным свойством:

$$
\frac{P_{n}}{Q_{n}}(z)=\sum_{k=0}^{2 n-1} \frac{c_{k}}{z^{k+1}}+\frac{A_{n}}{z^{2 n+1}}+\cdots,
$$

тем самьм,

$$
\left(f-\frac{P_{n}}{Q_{n}}\right)(z)=O\left(\frac{1}{z^{2 n+1}}\right)
$$

Соотношение (2.2) единственным образом определяет рациональную функцию $P_{n} / Q_{n}$ в классе всех рациональных дробей порядка не выше $n$. Такая дробь доставляет наилучшее локальное приближение ряда (2.1) в указанном классе функций. Это свойство служит определением диагональных аппроксимачий Паде $[n / n]_{f}=P_{n} / Q_{n}$ для произвольного (формального или сходяшегося) ряда (2.1).

В [30] Чебьшёв впервые изучил формальные разложения в непрерьвные дроби для функций из фактически введенного им класса

$$
\widehat{\mu}(z):=\int_{S} \frac{d \mu(\zeta)}{z-\zeta},
$$

где положительная мера $\mu$ с носителем $S=S_{\mu} \subset \mathbb{R}$ имеет вид: $d \mu(x)=\theta(x) d x$, $\theta \geqslant 0$ - суммируемая функция, вес. Именно на этом пути Чебьшёв открыл и исследовал общие ортогональные многочленьи, соответствующие произвольному весу на $\mathbb{R}:$ такие многочлены естественным образом возникают как знаменатели $Q_{n}$ подходящих дробей функций $\widehat{\mu}(z)$ вида (2.3).

Одним из первых результатов общего характера о сходимости диагональных аппроксимаций Паде аналитических функций является классическая теорема Маркова [19], полученная им в терминах чебьшёвских непрерьвных дробей.

Теорема Маркова. Для функиии $\widehat{\mu}$ вида (2.3) с носителем $S_{\mu} \Subset \mathbb{R}$, состоящим из бесконечного множества точек, диагональные аппроксимачии Паде $[n / n]_{\widehat{\mu}}$, построенные по коэффичиентам разложсения $\widehat{\mu}$ в ряд Лорана в точке $z=\infty$, сходятся $\kappa \widehat{\mu}$ равномерно внутри области $\overline{\mathbb{C}} \backslash[a, b]$, әде $[a, b]-$ минимальный отрезок вещественной оси, содержащий $S_{\mu}$.

Тем самым, любая марковская функиия (т.е. функция вида $(2.3)$ с $S_{\mu} \Subset \mathbb{R}$ ) может быть восстановлена вне выпуклой оболочки $\widehat{S_{\mu}}=[a, b]$ носителя меры по коэффициентам своего лорановского разложения в точке $z=\infty$ (моментам меры $\mu$ ).

K теореме Маркова непосредственно примыкают результаты А. А. Гончара [31] и Е. А. Рахманова [32] о сходимости диагональных аппроксимаций Паде для мероморфныз функций $f$ вида

$$
f=\widehat{\mu}+r
$$


где $\widehat{\mu}$ - марковская функция, $r$ - рациональная функция, голоморфная на $[a, b](r \in$ $\mathbb{C}(z) \cap \mathscr{H}[a, b], f$ - "рациональное возмущение" $\widehat{\mu})$. Конструкция Паде существенно нелинейна, поэтому исследование сходимости таких аппроксимаций для функций вида (2.4) - нетривиальная задача. В теореме Гончара утверждается, что диагональные аппроксимации Паде $[n / n]_{f}$ сходятся равномерно в сферической метрике к функции $f$ внутри области $\overline{\mathbb{C}} \backslash[a, b]$ при условии, что $S_{\mu}=[a, b] u \mu^{\prime}(x)=d \mu / d x>0$ почти всюду на $[a, b]$, а функиия $r \in \mathbb{C}(z)$ и голоморфна на $[a, b]$. В работе Рахманова аналогичный результат о равномерной сходимости $[n / n]_{f}$ в сферической метрике вне $[a, b]$ установлен в предположении, что $\widehat{\mu}-$ произвольная марковская функиия, $a$ функиия $r \in \mathbb{R}(z)$ и голоморфна на $[a, b]$.

Тем самым, диагональные аппроксимации Паде в классе функций вида (2.4) при определенных условиях эффективно решают задачу мероморфного продолжения в область $\overline{\mathbb{C}} \backslash[a, b]$ функции, заданной своим разложением в ряд Лорана в бесконечно удаленной точке: полюсы этих аппроксимаций локализуют отрезок $[a, b]$ и особые точки (полюсы и их кратности) функции $f$ в $\overline{\mathbb{C}} \backslash[a, b]$, а сами аппроксимации осушествляют мероморфное продолжение ряда $f$ в $\overline{\mathbb{C}} \backslash[a, b]$.

Тот факт, что в теореме Маркова речь идет о равномерной сходимости не в $\overline{\mathbb{C}} \backslash S_{\mu}$ - области голоморфности функции $\widehat{\mu}$, а лишш вне вьпуклой оболочки $\widehat{S_{\mu}}$ носителя меры, связан с сушеством дела: непосредственно из открытого Чебьшёвым свойства ортогональности относительно меры $\mu$ знаменателей диагональных аппроксимаций Паде функции вида (2.3), вытекает, что если носитель меры $\mu$ состоит из нескольких непересекающихся отрезков, то в лакунах между этими отрезками могут располагаться полюсы рациональной функции $[n / n]_{\widehat{\mu}}$. Более того, как показано в работе, "в общем положении" множество предельных точек полюсов рациональных функций $[n / n]_{\widehat{\mu}}$ - нулей соответствуюших ортогональных полиномов - coвnaдаem $c \widehat{S_{\mu}}$, что делает принципиально невозможной равномерную сходимость $[n / n]_{\widehat{\mu}}$ внутри $\overline{\mathbb{C}} \backslash S_{\mu}$ в классе всех марковских функций (2.3). Аналогичная ситуация, когда полюсы диагональных аппроксимаций Паде имеют предельные точки в области мероморфности заданной функции вида (2.4), не соответствующие полюсам рациональной функции $r$, имеет место и в условиях теоремы Рахманова. Если же в этом случае допустить, что $r \in \mathbb{C}(z)$, а $S_{\mu}$ состоит в точности из двух отрезков, то, как показано в [22], сушествует аналитическая дуга $\gamma$, соединяюшая эти отрезки и лежашая в $\overline{\mathbb{C}} \backslash[a, b]$, такая, что равномерная сходимость аппроксимаций Паде $[n / n]_{f}$ к функции $f$ в сферической метрике имеет место в $\overline{\mathbb{C}} \backslash\left(S_{\mu} \cup \gamma\right)$. При этом если число $\omega_{1}(\infty)$ - гармоническая мера первого отрезка относительно области $\overline{\mathbb{C}} \backslash S_{\mu}$, вьчисленная в бесконечно удаленной точке, - иррационально, то сходимость не имеет места ни в какой области, пересекающейся с $\gamma$; в противном случае существует конечное множество точек $\mathscr{P}$ такое, что равномерная сходимость имеет место в $\overline{\mathbb{C}} \backslash\left(S_{\mu} \cup \mathscr{P}\right)$. Дуга $\gamma$ полностью определяется "геометрией” задачи: она зависит только от $S_{\mu}$ и расположения полюсов функции $r$ и не зависит от меры $\mu$ и вычетов функции $r$ в полюсах.

В общем же случае, когда носитель меры $\mu$ в (2.3) не лежит на прямой, предельные точки полюсов диагональных аппроксимаций Паде могут составлять аналитические дуги в области $D=\overline{\mathbb{C}} \backslash S_{\mu}$ и даже быть всюду плотными в $\overline{\mathbb{C}}$. Более точно, по некоторьм подпоследовательностям полюсы аппроксимаций Паде могут сходиться к любой наперед заданной точке аналитической дуги или, соответственно, к произвольной 
точке $\overline{\mathbb{C}}$. В такой ситуации принципиальным становится вопрос о том, может ли какой-либо полюс аппроксимаций Паде иметь предел (а не только предельную точку) по всей последовательности $n \in \mathbb{N}$, отличньй от полюса функции $f$. Этот вопрос непосредственно связан с задачей о восстановлении диагональю Паде дивизора полюсов мероморфной в $\overline{\mathbb{C}} \backslash S_{\mu}$ функции.

Основная цель второй главы статьи - доказательство некоторых результатов о восстановлении главной диагональю Паде дивизора полюсов мероморфных функций. Методы, используемые нами во второй главе, таковы, что эти результаты вытекают непосредственно из положительного решения задачи о сушествовании для произвольной функции из определенного класса некоторой подпоследовательности диагональных апроксимаций Паде, равномерно сходящейся в сферической метрике к этой функции в ее области мероморфности.

Наиболее обший результат о сходимости диагональных аппроксимаций Паде был получен Шталем [34]. Для некоторого естественного класса функций вида (2.4), содержашего все алгебраические функиии, Шталь доказал сходимость диагональных последовательностей аппроксимаций Паде по емкости. Сходимость диагональных аппроксимаций Паде непосредственно связана с асимптотикой их знаменателей - многочленов, неэрмитово ортогональных относительно меры $\mu$ на дугах, составляющих $S$. Подход Шталя фактически основан на изучении так назьваемой “слабой” асимптотики таких многочленов.

Во настоящей главе мы развиваем подход Наттолла, в основе которого - изучение "сильной" (т.е. типа Бернштейна-Сегё) асимптотики знаменателей аппроксимаций Паде. В рамках такого подхода Наттолл [9], [35] получил результат о сходимости по емкости последовательности диагональных аппроксимаций Паде функций из класса (2.4) при условии, что носитель $S$ меры $\mu$ - компакт минимальной емкости, соответствующий некоторой многозначной функции вида $\sqrt{\left(z-e_{1}\right) \cdots\left(z-e_{2 \mathrm{~g}+2}\right)}$, где точки $e_{1}, \ldots, e_{2 \mathrm{~g}+2} \in \mathbb{C}$ попарно различны, g $\geqslant 0$. Сама же мера в этом случае предполагается достаточно гладким комплекснозначным весом на $S$. Этот класс функций содержит общие гиперэллиптические функции.

Отметим, что случай әллиптической функции $f \in \mathscr{H}(\infty)$ вида

$$
f(z)=\sqrt{\left(z-e_{1}\right) \cdots\left(z-e_{4}\right)}-z^{2}+z\left(e_{1}+\cdots+e_{4}\right) / 2,
$$

где точки $e_{1}, \ldots, e_{4} \in \mathbb{C}$ попарно различны и выбрана та ветвь корня, старший член которой в окрестности бесконечно удаленной точки равен $z^{2}$, был исследован Дюма [36] в терминах непрерывных дробей. Он показал, что если некоторые числовые параметры, связанные только с взаимньм расположением точек $e_{1}, \ldots, e_{4}$, рационально независимы, то множество полюсов диагональных аппроксимаций Паде всюду плотно в $\overline{\mathbb{C}}$, т.е. последовательность $[n / n]_{f}$ не может равномерно сходиться ни в какой подобласти $\mathbb{C}$. Однако эта последовательность в силу теоремы Наттолла сходится по емкости в дополнении к разрезам "минимальной емкости".

Здесь мы предполагаем, что мера $\mu$ такова, что функция $\widehat{\mu}$ представляется в виде

$$
\widehat{\mu}(z)=\frac{1}{2 \pi i} \int_{S} \frac{\rho(\zeta)}{z-\zeta} \frac{d \zeta}{\sqrt{h(\zeta)}}=: \widehat{\rho}(z ; h),
$$

где $h=h_{2 \mathrm{~g}+2} \in \mathbb{C}[z]-$ некоторьй полином четной степени $2 \mathrm{~g}+2, \mathrm{~g} \geqslant 0$, с единичным старшим коэффициентом, все корни $\left\{e_{j}\right\}$ которого - простые, $S=S(h)-$ 
компакт, имеюший минимальную емкость среди всех компактов в $\mathbb{C}$, в дополнении к которьм выделяется однозначная ветвь квадратного корня $\sqrt{h(z)}, S=\bigcup_{j=1}^{\mathrm{g}+1} S_{j}$, $S_{j}=\operatorname{arc}\left(e_{2 j-1}, e_{2 j}\right)$ (дуги $S_{j}$, вообще говоря, могут пересекаться); под $\sqrt{h(\zeta)}, \zeta \in S$, понимаются "верхние" граничные значения на $S$ той ветви корня, которая определяется условием $\sqrt{h(z)} / z^{\mathrm{g}+1} \rightarrow 1$ при $z \rightarrow \infty$, т.е. граничные значения на “левом" берегу каждой дуги $S_{j}$, ориентированной от $e_{2 j-1}$ к $e_{2 j}$; кроме того, комплекснозначная функция $\rho(\zeta)$ не имеет нулей и удовлетворяет на $S$ условию Гёльдера с некоторым показателем $\alpha \leqslant 1$ (будем говорить, что $\rho$ Н-непрерьвна на $S$ и писать $\rho \in \mathrm{H}(\alpha ; S)$, $0<\alpha \leqslant 1)$. Такую функцию $\rho$ будем назьвать допустимым весом. В частном случае, когда $\rho(\zeta)$ - рациональная функция переменной $\zeta(\rho \in \mathbb{C}(\zeta))$, не имеющая на $S$ ни нулей, ни полюсов, функция $\widehat{\rho}(\cdot ; h)$ - гиперэллиптическая функция, определенная на гиперэллиптической римановой поверхности $\mathfrak{R}=\mathfrak{R}(h)$ рода $\mathrm{g}=\mathrm{g}(h)$, заданной уравнением $w^{2}=h(z): \widehat{\rho}(z ; h)=r_{1}(z) w+r_{2}(z)$, где $r_{1}, r_{2} \in \mathbb{C}(z) ;$ тем самым, $\widehat{\rho}(\cdot ; h) \in \mathbb{C}(z, w)$. Наконец, отметим, что в соответствии с выбранной нами ветвью функции $\sqrt{h}$ марковскому случаю положительной меры $\mu$ на $\mathbb{R}$ соответствует такой вес $\rho$ с носителем на $\mathbb{R}$, для которого вьполняется неравенство $\rho /(i \sqrt{h})>0$ на $S$.

Вопрос о сходимости диагональных и близких к ним последовательностей аппроксимаций Паде функщий вида (2.5) изучался в [35], где дано обобшение на случай произвольного $h \in \mathbb{C}[z]$ четной степени и комплексного веса $\rho \neq 0$ классического anпроксимационного метода Бернштейна-Сегё [37], основанного на явных формулах для полиномов, ортогональных на отрезке $[-1,1]$ относительно веса $\rho$ такого, что $1 / \rho \in \mathbb{R}[x]$; в этом случае $\widehat{\rho}(z)=r_{1}(z) w+r_{2}(z)$, где $r_{1}, r_{2} \in \mathbb{C}(z)$, a $w^{2}=z^{2}-1$. $\mathrm{B}[35]$ была доказана сходимость по емкости таких аппроксимаций к функции $\widehat{\rho}$ равномерно внутри (на компактных подмножествах) области $D=\overline{\mathbb{C}} \backslash S$ при несколько более общих, чем в настоящей работе, условиях на вес $\rho$, но также в предположении, что компакт минимальной емкости имеет ровно g +1 компоненту связности (т.е. дуги $S_{j}$ попарно не пересекаются); в работе мы накладываем это условие на взаимное расположение точек $e_{1}, \ldots, e_{2 \mathrm{~g}+2}$ как первое условие "общего положения". Как уже отмечалось, использование понятия сходимости по емкости связано с существом дела: хотя "почти все" полюсы диагональных аппроксимаций Паде $[n / n]_{\widehat{\rho}}$ функции $\widehat{\rho}$ "стягиваются" к $S$, конечное число полюсов рациональных функций $[n / n]_{\widehat{\rho}}$ может иметь предельные точки в $D$ - области голоморфности функции $\widehat{\rho}$. Наличие таких “лишних" полюсов и является по существу единственным препятствием для равномерной сходимости диагональных аппроксимаций Паде функций вида (2.5). Таким образом, вопрос о существовании равномерно сходящейся подпоследовательности аппроксимащий Паде сводится к вопросу о сушествовании подпоследовательности, по которой эти аппроксимации не имеют полюсов в $D$. В свою очередь, поскольку знаменатели диагональных аппроксимаций Паде функщии (2.5) - многочлены, неэрмитово ортогональные на $S$ относительно веса $\rho(\zeta) / \sqrt{h(\zeta)}$, естественным образом приходим к задаче о сильной асимптотике таких ортогональных полиномов - полиномов Паде. Наиболее общие результаты об асимптотике такого типа для многочленов, эрмитово ортогональных на системе кривых и дуг в $\mathbb{C}$ относительно вещественного веса $\rho \geqslant 0$, удовлетворяющего условию Сегё, получены Видомом [58] (см. также [59]); ответ дан им в терминах решений некоторых экстремальных весовых (относительно $\rho$ ) задач, в которых условие вешественности и неотрищательности веса $\rho$ играет ключевую роль. Таким образом, полученные в настоящей работе результаты “пересекаются" с результа- 
тами Видома только для классического случая - полиномов Ахиезера [38], [60], [61]: все корни $e_{1}, \ldots, e_{2 \mathrm{~g}+2}$ полинома $h(z)$ - вешественные, т.е. $S$ состоит из конечного числа отрезков вешественной оси $\mathbb{R}$, а $\rho(\zeta) /(i \sqrt{h(\zeta)})>0$ на $S$. Отметим, что и в этой ситуации нам удалось сделать некоторое уточнение к результату Видома (см. ниже предложения 2.3 и 2.4 ).

В целом же рассматриваемая здесь задача об асимптотике полиномов Паде потребовала нового, иного чем у Видома, подхода, которьй впервые был предложен Наттоллом в [39]: опираясь на некоторые изученные к тому времени частные случаи, он высказал гипотезу, что асимптотику полиномов Паде можно адекватно описать в терминах решений некоторой краевой задачи Римана, поставленной на расширенной комплексной плоскости с разрезами по аналитическим дугам, составляюшим компакт минимальной емкости $S$. Эту идею Наттолл сумел реализовать [40], [41] лишш в уже хорошо изученном классическом случае g $=0$ (т.е. при $S=[-1,1]$ ), получив некоторое сингулярное уравнение на полиномы Паде (т.е. на многочлены, ортогональные на отрезке $[-1,1])$. В дальнейшем подобное уравнение было получено для некоторых частных случаев, в том числе соответствующих $\mathrm{g}=1$ (см. [42], где имеются дальнейшие ссылки).

В [29] нам удалось при некоторых достаточно общих геометрических условиях на расположение точек $e_{1}, \ldots, e_{2 \mathrm{~g}+2}$ (точнее, в случае "общего положения") фактически охарактеризовать асимптотическое поведение полиномов Паде в терминах решения краевой задачи Римана. Анализ сушествования и нахождение "явного" вида решения такой задачи дан в [29] в терминах обладающего специальными свойствами решения проблемы Якоби обращения абелевых интегралов. Наконец, используя свойства полученного решения этой задачи Римана, мы вьводим сингулярное интегральное уравнение (см. ниже (2.40)) для полиномов Паде, которое справедливо при любом $\mathrm{g} \in \mathbb{N}$.

В конечном итоге, этот новый подход, основанньй на краевой задаче Римана и сингулярном уравнении, позволяет обойтись без традиционного аппроксимационного метода Бернштейна-Сегё, о котором говорилось вьше.

Отметим, что первый положительный результат, касаюшийся гипотезы БейкераГамелля-Уиллса (см. [13]), был получен Шталем [43] для гиперэллиптических функuй

$$
f(z)=r_{1}(z) w+r_{2}(z)
$$

$w^{2}=h(z), r_{1}, r_{2} \in \mathbb{C}(z)$, при некотором дополнительном условии на расположение нулей и полюсов рациональных функций $r_{1}, r_{2}$ и также в условиях "общего положения"; непосредственно из полученного ниже тождества (2.9) вытекает, что “общее положение" из работы Шталя [43] эквивалентно используемому в настоящей работе. Тем самым, из результатов настоящей работы следует, что гипотеза Бейкера-Гамелля-Уиллса справедлива и для находящейся в “общем положении” функции из следующего класса, более общего, чем класс гиперэллиптических функций:

$$
f(z)=\widehat{\rho}(z ; h)+r(z), \quad z \in \overline{\mathbb{C}} \backslash S,
$$

где $\widehat{\rho}$ - вида $(2.5), \rho \neq 0$ и Н-непрерьвна на $S=S(h), h \in \mathbb{C}[z], \operatorname{deg} h=0(\bmod 2)$, а $r \in \mathbb{C}(z) \cap \mathscr{H}(S)$. Класс таких функций $f$ вида (2.6) будем назьвать классом Наттолла и обозначать через $\mathscr{N}$; если же, кроме того, в $(2.6)$ все нули полинома $h$ лежат 
на $\mathbb{R}$ и вес $\rho$ удовлетворяет неравенству $\rho / i \sqrt{h}>0$ на $S$, то будем писать $f \in \mathscr{N}^{\circ}$. Для функции $f \in \mathscr{N}$ соответствующую область $\overline{\mathbb{C}} \backslash S$ будем обозначать через $D=D_{f}$.

1.2. Пусть $f$ - ряд $(2.1),\left\{[n / n]_{f}\right\}_{n \in \mathbb{N}}-$ соответствуюшая ему последовательность диагональных аппроксимаций Паде ("главная диагональ" Паде).

Для произвольной точки $a \in D_{f}$ определим следующую характеристику $k_{f}(a) \in$ $\mathbb{Z}_{+}$. Пусть $\mathscr{P}_{n}(f)=\left\{\zeta_{1, n}, \ldots, \zeta_{n^{\prime}, n}\right\}, n^{\prime} \leqslant n,-$ множество всех конечных полюсов рациональной функции $[n / n]_{f}$; будем считать, что полюсы занумерованы в порядке неубьвания их расстояния от точки $a: \zeta_{j, n}=\zeta_{j, n}(a)$. Тогда

$$
k_{f}(a)=\sup \left\{k \in \mathbb{N}: \zeta_{k, n}(a) \rightarrow a, n \rightarrow \infty\right\}
$$

если множество индексов $k$ в последней формуле пусто, то полагаем $k_{f}(a)=0$. Очевидно, $0 \leqslant k_{f}(a) \leqslant \infty$, причем при $f \in \mathcal{N}$ из теоремы Наттолла вытекает, что величина $k_{f}(a) \geqslant 1$, если $a$ - полюс $f$; из приводимых ниже результатов вытекает, что в "общем положении" для $f \in \mathscr{N}$ величина $k_{f}(a)<\infty$, если $a \in D_{f}$.

Для произвольной точки $a \in D_{f}$ положим: $p_{f}(a)$ - кратность полюса функции $f$ в этой точке, если $a-$ полюс $f$, и $p_{f}(a)=0$ в противном случае; тем самым, $\left\{\left(a, p_{f}(a)\right)\right.$, $\left.a \in \mathbb{C}: p_{f}(a) \geqslant 1\right\}$ - дивизор полюсов функции $f \in \mathscr{N}$ в области $D_{f}$.

Для функции $f \in \mathscr{N}^{\circ}$ “обшим положением” будем назьвать ситуацию, когда нули соответствующего полинома $h=h(z ; f)$, все лежащие на одной прямой, таковы, что числа $\omega_{1}(\infty), \ldots, \omega_{\mathrm{g}+1}(\infty)$ - гармонические меры составляюших множество $S$ отрезков относительно области $D_{f}$, вычисленные в бесконечно удаленной точке, - paционально независимы. Нетрудно видеть, что такая ситуация действительно является "общим положением" в смысле работы [43]: если рассматривать аппроксимации Паде функции $f \in \mathcal{N}^{\circ}$, соответствуюшие разложению $f$ в ряд в некоторой точке $x \in \mathbb{R} \backslash S$, то в любом интервале $\ell \subset \mathbb{R}$ множество точек $x \in \ell$ таких, что числа $\omega_{1}(x), \ldots, \omega_{\mathrm{g}+1}(x)$ рационально независимы, имеет полную относительно $\ell$ линейную меру Лебега.

“Общее положение" в классе $\mathcal{N}$ определяется условиями $\left(C_{1}\right)-\left(C_{2}\right)$ (см. ниже).

Основньми результатами второй главы являются следующие теоремы.

Теорема 3. Пусть ряд (2.1) определяет функиию $f \in \mathscr{N}^{\circ}$ и нули полинома $h=h(z ; f)$, все лежащие на одной прямой, находятся в "общем положении". Тогда $p_{f}(a)=k_{f}(a)$ для любой точки $a \in D_{f}$.

ТЕОРема 4. Пусть ряд (2.1) определяет функцию $f \in \mathscr{N}$, причем нули полинома $h=h(z ; f)$ находятся в “общем положсени". Тогда $p_{f}(a)=k_{f}(a)$ для любой точки $a \in D_{f}$.

Таким образом, в условиях теорем 3 и 4 каждьй полюс $a$ функции $f$ в $D$ притягивает ровно $k=p_{f}(a)$ полюсов аппроксимаций Паде $[n / n]_{f}, n \in \mathbb{N}$ :

$$
\zeta_{j, n}(a) \rightarrow a, \quad j=1, \ldots, k, \quad n \rightarrow \infty ; \quad \varlimsup_{n \rightarrow \infty}\left|\zeta_{k+1, n}(a) ; a\right|>0,
$$

где $|\cdot ; \cdot|-$ расстояние в сферической метрике между двумя точками из $\overline{\mathbb{C}}$.

Иначе говоря, диагональные аппроксимации Паде восстанавливают дивизор полюсов в $D_{f}$ произвольной находящейся в “обшем положении” мероморфной функции $f$ 
из класса $\mathscr{N}$ или $\mathscr{N}^{\circ}$. Этот результат в определенном смысле дополняет теорему Наттолла о сходимости по емкости диагональных аппроксимаций Паде произвольной функции $f \in \mathcal{N}$. Действительно, непосредственно из этой теоремы Наттолла вытекает, что мера, ассоциированная с нулями знаменателя $Q_{n}$, слабо сходится к равновесной мере компакта $S$; тем самым, в классе функций $\mathscr{N}$ главная диагональ Паде восстанавливает носитель $S$.

Отметим, что из метода доказательства теорем 3 и 4 вытекает следуюший формально более общий, чем эти теоремы, результат.

СлЕДСТВИЕ. В условиях теоремы 3 или 4 главная диагональ Паде восстанавливает дивизор (нулей и полюсов) функиии $f$ в $D_{f}$.

Как уже отмечалось, эти результаты являются прямыми следствиями теорем о существовании в “общем положении” для функщий классов $\mathscr{N}^{\circ}$ и $\mathscr{N}$ равномерно сходяшихся в сферической метрике подпоследовательностей диагональных апшроксимаций Паде. Более точно, схема доказательства теорем 3 и 4 состоит в вьводе следующих импликаций:

1) сушествование обладаюшего спешиальными свойствами решения проблемы Якоби обрашения абелевых интегралов $\Longrightarrow$

2) сушествование решения некоторой краевой задачи Римана $\Longrightarrow$

3) сингулярное интегральное уравнение на полиномы Паде $\Longrightarrow$

4) существование сильной асимптотики полиномов Паде $\Longrightarrow$

5) равномерная сходимость в сферической метрике диагональных аппроксимаций Паде $\Longrightarrow$

6) справедливость теорем 3 и 4.

Для функции $f \in \mathscr{N}$ будем говорить, что подпоследовательность диагональных аппроксимаций Паде $[n / n]_{f}, n \in \Lambda$, равномерно в сферической метрике сходится к функции $f$ внутри области $D_{f}$, если для любого компакта $K \Subset D_{f}$

$$
\sup _{z \in K}\left|f(z) ;[n / n]_{f}(z)\right| \rightarrow 0, \quad n \rightarrow \infty, \quad n \in \Lambda
$$

где $|\cdot ; \cdot|-$ как и выше, расстояние в сферической метрике между двумя точками из $\overline{\mathbb{C}}$.

ПРЕДЛОЖЕНИЕ 2.1. Если все точки $e_{1}, \ldots, e_{2 g+2}$ расположены на одной прямой и числа $\omega_{1}(\infty), \ldots, \omega_{\mathrm{g}+1}(\infty)$ - гармонические меры отрезков, составляющих множество $S$, относительно области $D$ - рачионально независимы, то для любой функции $f(z)=\widehat{\rho}(z ; h)+r(z)$, где $\rho-$ допустимый в классе $\mathscr{N}^{\circ}$ вес на $S$, а $r \in \mathbb{C}(z) \cap \mathscr{H}(S)$, существует подпоследовательность $\Lambda \subset \mathbb{N}$ такая, что

$$
[n / n]_{f} \rightarrow f, \quad n \rightarrow \infty, \quad n \in \Lambda
$$

равномерно в сферической метрике внутри D. 
ПРЕДЛОЖЕНИЕ 2.2. Если точки $e_{1}, \ldots, e_{2 \mathrm{~g}+2}$ находятся в "общем положении" $\left(C_{1}\right)-\left(C_{2}\right)$ (см. ниже), то для любой функиии $f(z)$ вида

$$
f(z)=\widehat{\rho}(z ; h)+r(z),
$$

где вес $\rho$ - допустимый, а $r \in \mathbb{C}(z) \cap \mathscr{H}(S)$, существует бесконечная подпоследовательность $\Lambda \subset \mathbb{N}$ такая, что $\operatorname{deg} Q_{n}=n$ для $n \in \Lambda$ и

$$
[n / n]_{f} \rightarrow f, \quad n \rightarrow \infty, \quad n \in \Lambda,
$$

равномерно в сферической метрике внутри $D$.

Как уже отмечалось, наш подход к изучаемой проблеме таков, что в основе исследования вопроса о равномерной сходимости диагональных аппроксимаций Паде лежит доказательство формулы сильной асимптотики для знаменателей этих рациональных функций - полиномов Паде. Точнее, пусть $f-$ ряд $(2.1)$ и полиномы $P_{n}(z)=P_{n}(z ; f)$ и $Q_{n}(z)=Q_{n}(z ; f), P_{n}, Q_{n} \in \mathbb{C}[z], \operatorname{deg} P_{n} \leqslant n-1, \operatorname{deg} Q_{n} \leqslant n, Q_{n} \not \equiv 0$, удовлетворяют следуюшему условию:

$$
\left(Q_{n} f-P_{n}\right)(z)=O\left(\frac{1}{z^{n+1}}\right), \quad z \rightarrow \infty .
$$

Тогда $[n / n]_{f}(z)=P_{n}(z) / Q_{n}(z)$. Напомним, что рациональная функция $[n / n]_{f}(z)$ однозначно определяется условием (2.2) максимальности касания ряда $f$ в классе всех рациональных функций порядка не вьше $n$. Как обычно, индекс $n$ назовем нормальнылм, если $\operatorname{deg} Q_{n}=n$ для любого $Q_{n}$, удовлетворяющего условию $(2.7)$; в таком случае нормировкой $Q_{n}(z)=z^{n}+\cdots$ полином Паде определяется однозначно. Для функции $f=\widehat{\rho}$ вида $(2.5)$ полиномы $Q_{n}$ неэрмитово ортогональны на $S=S(h)$ относительно веса $\rho / \sqrt{h}$.

Справедливо следующее

ПРЕДЛОЖЕНИЕ 2.3. Если выполняются условия теоремы 3 или 4 на полином $h$, то для любого допустимого в классе $\mathscr{N}^{\circ}$ или $\mathscr{N}$ веса $\rho$ на $S$ существует бесконечная подпоследовательность $\Lambda \subset \mathbb{N}$ такая, что при $n \in \Lambda$ индекс $n$ нормальный $и$

(i) $Q_{n}(z ; \widehat{\rho})=\operatorname{cap}(S)^{n} \Phi^{n}(z) F_{n}(z ; \rho)(1+o(1)), n \in \Lambda$, равномерно внутри области $D$, где $\Phi(z)$ - отображсающая функция, $F_{n}(z ; \rho)$ - многозначная аналитическая функиия с однозначным модулем, нормированная условием $\left|F_{n}(\infty ; \rho)\right|=1$ и не имеющая нулей в $D$;

(ii) $[n / n]_{\widehat{\rho}} \rightarrow \widehat{\rho}, n \in \Lambda$, равномерно внутри области $D$;

(iii) при $n \in \Lambda$ рациональная функция $[n / n]_{\widehat{\rho}}(z)$ интерполирует функцию $\widehat{\rho}(z)$ дополнительно ${ }^{2}$ в g конечных точках области $D$.

Изучение асимптотических свойств полиномов Паде, в свою очередь, основано на исследовании некоторой краевой задачи Римана для многосвязной области $D$.

Как уже отмечалось, компакт минимальной емкости для гиперэллиптического случая состоит из конечного числа замкнутых, вообще говоря, пересекающихся аналитических дуг $S_{j}$ (см., например, [23], [39], а также [62], [63]). Будем считать, что вьполнено первое условие "общего положения":

\footnotetext{
${ }^{2}$ Напомним, что если индекс $n$ нормален, для рациональной функции $[n / n]_{f}$ выполняется интерполяционное соотношение (2.2).
} 
$\left(C_{1}\right)$ аналитические дуги $S_{j}, j=1, \ldots, \mathrm{g}+1$, составляющие компакт минимальной емкости $S$, не пересекаются.

При этом условии $D=\overline{\mathbb{C}} \backslash S-$ g-связная область в $\overline{\mathbb{C}}$. Через $S_{j}^{+}, S_{j}^{-}$будем обозначать соответственно "верхний” и "нижний” берега дуг $S_{j}=\operatorname{arc}\left(e_{2 j-1}, e_{2 j}\right), j=$ $1, \ldots, \mathrm{g}+1$, составляюших $S$ и ориентированных согласованно с ориентацией $S$ как гранищы области $D$ : область $D$ остается слева при обходе $j$-й компоненты от точки $e_{2 j-1}$ к точке $e_{2 j}$ по "верхнему" берегу и обратно от $e_{2 j}$ к $e_{2 j-1}$ по "нижнему". Пусть $\Gamma=S^{+} \cup S^{-}$; отметим, что кривая $\Gamma=\bigcup_{j=1}^{\mathrm{g}+1} \Gamma_{j}, \Gamma_{j}=\operatorname{arc}\left(e_{2 j-1}, e_{2 j}\right) \cup$ $\operatorname{arc}\left(e_{2 j}, e_{2 j-1}\right)$, рассматриваемая как кривая на римановой поверхности $\mathfrak{R}$, заданной уравнением $w^{2}=h$, состоит из конечного числа непересекающихся аналитических жордановых контуров $\Gamma_{j}, j=1, \ldots, \mathrm{g}+1$. Через $\mathscr{C}(D)$ обозначим класс всех функций, мероморфных в $D$ и Н-непрерьвно продолжимых на $S^{+} \cup S^{-}$(т.е. с граничными значениями, удовлетворяюшими условию Гёльдера с показателем $\leqslant 1)$. Предельные значения функций такого класса на $S^{+}$и $S^{-}$будем обозначать соответственно при помощи “+” и "-”. Пусть на $S$ задана комплекснозначная Н-непрерьвная функция $\rho \neq 0$ (т.е. допустимьй вес). Как уже отмечалось, наш подход к изучению асимптотических свойств полиномов Паде основан на исследовании некоторой краевой задачи для многосвязной области $D$.

Для фиксированного $n \in \mathbb{N}, n \geqslant \mathrm{~g}$, рассмотрим следуюшую краевую задачу Римана.

ЗАДАчА $(\mathscr{R})$. Найти пару функций $\varphi_{n}, \psi_{n} \in \mathscr{C}(D)$ таких, что

(i) $\varphi_{n}$ имеет полюс порядка $n$ в точке $z=\infty$ и не имеет ни других полюсов, ни нулей в $D$;

(ii) $\psi_{n}$ имеет нуль кратности $n-\mathrm{g}$ в точке $z=\infty$ и еще ровно g “свободных" нулей в $D^{*}=D \backslash\{\infty\} ;$ других нулей или полюсов функция $\psi_{n}$ не имеет;

(iii) на множестве $S$ выполняются краевые условия $\rho \varphi_{n}^{+}=\psi_{n}^{-}, \rho \varphi_{n}^{-}=\psi_{n}^{+}$.

Пару таких функций $\varphi_{n}=\varphi_{n}(z ; \rho), \psi_{n}=\psi_{n}(z ; \rho)$, нормированных условием $\varphi_{n}(z) / z^{n} \rightarrow 1$ при $z \rightarrow \infty$, назовем решением задачи $(\mathscr{R})$. Ниже будет показано, что если при введенной нормировке решение этой задачи сушествует, то оно единственно.

Исследованию вопроса сушествования решения задачи $(\mathscr{R})$ посвяшен $\S 3$ этой главы. Задачу $(\mathscr{R})$ удается изучить, рассматривая ее фактически как краевую задачу Римана для кусочно-мероморфной функции на двулистной гиперэллиптической римановой поверхности $\mathfrak{R}: w^{2}=h(z)$. Отметим, что, хотя в [64] рассмотрен формально более общий случай произвольной компактной римановой поверхности, в нашей ситуации задача приобретает определенную специфику из-за того, что краевое условие задается на кривой $\Gamma \subset \mathfrak{R}$, каноническая проекция которой на $\mathbb{C}$ есть компакт минимальной емкости. Как и в [64], анализ сушествования и структуры решения задачи будет проведен с помошю анализа сушествования обладающего определенными свойствами решения проблемы обращения Якоби (см. $\S 4)$.

Наш подход к изучению асимптотических свойств полиномов Паде $Q_{n}(z)$ основан на свойствах решения задачи $(\mathscr{R})$ : в работе фактически доказано, что при вьполнении $\left(C_{1}\right)$ достаточным условием существования по некоторой подпоследовательности сильной асимптотики нужного вида для полиномов Паде является разрешимость по этой подпоследовательности задачи $(\mathscr{R})$. Точнее, имеет место следующее 
ПРЕДЛОЖЕНИЕ 2.4. Если для некоторой последовательности $\Lambda \subset \mathbb{N}$ задача $(\mathscr{R})$ имеет решение $\varphi_{n}, \psi_{n}, n \in \Lambda$, причем все предельные точки "свободных" нулей функций $\psi_{n}$ принадлежсат области $D^{*}=D \backslash\{\infty\}$, mо $\operatorname{deg} Q_{n}=n$ для достаточно больших $n \in \Lambda$ и при нормировке полиномов Паде условием $Q_{n}(z ; \widehat{\rho})=z^{n}+\cdots$ для $n \in \Lambda$ имеем:

(i) $Q_{n}(z ; \widehat{\rho})=\varphi_{n}(z ; \rho)(1+o(1))$ равномерно внутри области $D$;

(ii) $Q_{n}(\zeta ; \widehat{\rho})=\varphi_{n}^{+}(\zeta ; \rho)+\varphi_{n}^{-}(\zeta ; \rho)+o(1)$ равномерно на $S$;

(iii) если вес $\rho$ аналитичен на каждой дуге $S_{j}$, то в (i)-(ii) имеем: $o(1)=o\left(q^{n}\right)$, $0<q<1 ;$ для $\rho \in \mathrm{H}(\alpha ; S)$ в (i)-(ii) имеем: $o(1)=O\left((\log n) / n^{\alpha}\right)$.

Отметим, что непосредственно из утверждения (i) этого предложения вытекает, что по подпоследовательности $\Lambda$ полюсы диагональных аппроксимаиий Паде не имеют предельных точек в $D$. Для частного случая, когда множество $S$ расположено на прямой, а $\rho(x)$ - допустимый вес, имеем: $Q_{n}(x ; \widehat{\rho})=T_{n}(x ; \rho)$ - полиномы Ахиезе$p a$, ортогональные на системе из $\mathrm{g}+1$ отрезков с весом $\rho / \sqrt{h}$, a $P_{n}(x ; \widehat{\rho})=U_{n-1}(x ; \rho)$ - соответствующие полиномы второго рода (см. [38]). Тем самым, для этого случая формула (i) (см. также предложение 2.3.(i)) является некоторым уточнением результата Видома $[58 ; \S \S 12,14]$.

Задачу $(\mathscr{R})$ удается исследовать в нужном для нас смысле пока только для двух случаев:

(I) все точки $e_{1}, \ldots, e_{2 \mathrm{~g}+2}$ лежат на одной прямой, а гармонические меры соответствуюших отрезков $\omega_{1}(\infty), \ldots, \omega_{\mathrm{g}+1}(\infty)$ рационально независимы (это так назьваемое "общее положение" в классе $\left.\mathscr{N}^{\circ}\right)$;

(II) имеет место "общее положение" в классе $\mathscr{N}$, которое кроме первого условия $\left(C_{1}\right)$ включает еще и второе условие $\left(C_{2}\right)$ :

$\left(C_{2}\right)$ периодьи ${ }^{3}$ комплексной функиии Грина $G_{D}(z, \infty)$, соответствующей компакту минимальной емкости $S$, рачионально независимы с числом $2 \pi i$.

Случай (I) не включается в (II): легко видеть, что в случае (I) не вьполняется условие $\left(C_{2}\right)$ "общего положения" - если все точки $\left\{e_{j}\right\}$ лежат на одной прямой, то все $b$-периоды комплексной функции Грина равны нулю (см. ниже).

На множестве всех Н-непрерывных на $S$ весов введем отношение эквивалентности. Два веса $\rho_{1}$ и $\rho_{2}$ назовем эквивалентными, если имеют место соотношения:

$$
\int_{S} \log \rho_{1}(\zeta) d \Omega_{k}^{+}(\zeta)=\int_{S} \log \rho_{2}(\zeta) d \Omega_{k}^{+}(\zeta) \quad(\bmod \pi i), \quad k=1, \ldots, \mathrm{g}
$$

здесь $d \Omega_{k}, k=1, \ldots, \mathrm{g},-a$-нормированный базис голоморфных абелевых дифференциалов на $\mathfrak{R}$ (см. $\S 2)$. Класс всех весов, эквивалентных весу $\rho$, будем обозначать через $[\rho]$.

Справедливо следуюшее

ПРЕДЛОЖЕНИЕ 2.5. Если выполняется условие (I) или условие (II), то для любого веса $\rho$ существует бесконечная подпоследовательность $\Lambda=\Lambda_{[\rho]} \subset \mathbb{N}$

\footnotetext{
${ }^{3}$ Напомним, что комплексная функция Грина имеет $2 \mathrm{~g}$ независимых периодов, которые все чисто мнимые.
} 
такая, что для каждого $n \in \Lambda$ задача $(\mathscr{R})$ имеет решение для всех допустимых весов из класса $[\rho]$, причем предельные точки “свободных" нулей функиий $\psi_{n}(z)$ принадлежсат области $D^{*}=D \backslash\{\infty\}$.

Тем самым, в условиях "обшего положения" (I) или (II) для $n \in \Lambda$ имеют место асимптотические соотношения (i) и (ii) предложения 2.4 .

На самом деле асимптотическое поведение полиномов Паде фактически полностью описывлется в терминах решения краевой задачи Римана $(\mathscr{R})$ при условии, что это решение понимается в несколько более широком, чем ранее, смысле. А именно, если предположить, что для каждого $n$, принадлежащего некоторой подпоследовательности $\Lambda^{\prime}$, сушествует пара функций $\varphi_{n}, \psi_{n}$ таких, что

$\left(\mathrm{i}^{\prime}\right)$ функция $\varphi_{n}$ имеет полюс порядка $n$ в точке $z=\infty$ и ровно $k \leqslant \mathrm{~g}$ "свободных" нулей в $D^{*}=D \backslash\{\infty\}$,

$\left(\mathrm{ii}^{\prime}\right) \psi_{n}$ имеет нуль кратности $n-\mathrm{g}$ в точке $z=\infty$ и еше ровно $\mathrm{g}-k$ "свободных" нулей в $D^{*}=D \backslash\{\infty\}$,

(iii) на множестве $S$ выполняются краевые условия $\rho \varphi_{n}^{+}=\psi_{n}^{-}, \rho \varphi_{n}^{-}=\psi_{n}^{+}$,

то при условии, что множества свободных нулей функций $\varphi_{n}$ и $\psi_{n}$ не пересекаются, по этой подпоследовательности $\Lambda^{\prime}$ имеет место аналог предложения 2.4. Отсюда уже легко вытекает

ПРЕДЛОЖЕНИЕ 2.6. В случае "общего положения" (I) множество предельных точек полюсов диагональных аппроксимаций Паде совпадает $c \widehat{S_{\mu}}=[a, b]$, а в случае (II) - со всей римановой сферой $\overline{\mathbb{C}}$.

Справедливость этого утверждения вытекает непосредственно из доказательства основной леммы $\S 3$ настоящей главы.

Наконец, отметим противоположный случай по отношению к случаю "общего положения", и тем самым не подпадаюший под действие вьшеуказанных теорем: периоды комплексной функции Грина $G_{D}(z, \infty)$ - рациональные кратные $2 \pi i$. Хорошо известно, что в этом случае поддается исследованию ситуация, когда вес $\rho \in \mathbb{C}[z]-$ полином степени $\mathrm{g}$, все корни которого лежат среди $e_{1}, \ldots, e_{2 \mathrm{~g}+2}(\rho$ делит полином $h$ : $\rho \mid h)$.

ТеОРема НАттолЛА-РАХманова. Если точки $e_{1}, \ldots, e_{2 \mathrm{~g}+2}$ maковы, что периоды комплексной функции Грина $G_{D}(z, \infty)$ - рациональнье кратные $2 \pi i$, а вес $\rho \in \mathbb{C}[z], \operatorname{deg} \rho=\mathrm{g} u \rho \mid h$, то по некоторой подпоследовательности $\Lambda \subset \mathbb{N}$ все индексы $n$ нормальны и полиномы Паде $Q_{n}(z)=z^{n}+\cdots$ допускают явное представление $Q_{n}(z)=\operatorname{cap}(S)^{n} \cdot\left(\Phi^{n}(z)+\Phi^{-n}(z)\right)$, где $\Phi(z)=\exp \left\{G_{D}(z, \infty)\right\}$.

Тем самым, для каждого $n \in \Lambda$ все нули полиномов $Q_{n}(z)$ лежсат на $S$. Подчеркнем, что $Q_{n}(z)$ - один и тот же для всех полиномов $\rho \mid h$. Ниже (см. п. 5.4) будет показано, что этот результат Наттолла-Рахманова фактически эквивалентен классической теореме Абеля о существовании полиномиального интегрируюшего множителя для дифференциала $d z / \sqrt{h(z)}$. В частности, полиномы Паде $P_{n}$ и $Q_{n}$ удовлетворяют уравнению Пелля $Q_{n}^{2} \rho-(h / \rho) P_{n}^{2} \equiv 1$. Отметим, что если в рассматриваемом случае все точки $\left\{e_{j}\right\}$ лежат на одной прямой, то при $n \in \Lambda$ имеем $Q_{n}(z)=T_{n}(z ; S)$ - полиномы Чебьшёва для системы отрезков $S=\bigcup_{j=1}^{\mathrm{g}+1}\left[e_{2 j-1}, e_{2 j}\right]$ : они ортогональны на $S$ относительно веса $\rho(x) / \sqrt{h(x)}$ и наименее уклоняются от нуля на $S$ в равномерной норме (cp. [65]). 


\section{§2. Основные определения и вспомогательные результаты}

2.1. Всюду в дальнейшем при использовании понятий, связанных с римановой поверхностью, мы в основном следуем [64], [66]-[68]. Будем считать, что точки $e_{1}, \ldots$, $e_{2 \mathrm{~g}+2}$ удовлетворяют первому условию общего положения $\left(C_{1}\right)$. Через $\mathfrak{R}$ обозначаем гиперэллиптическую риманову поверхность рода g, заданную уравнением $w^{2}=$ $h_{2 \mathrm{~g}+2}(z)$ и реализованную как двулистное разветвленное накрытие римановой сферы $\overline{\mathbb{C}}$ таким образом, что два листа представляют собой два экземпляра $\overline{\mathbb{C}}$ с разрезами по дугам, составляюшим компакт минимальной емкости; листы "склеиваются" друг с другом “накрест” по противоположным берегам соответствуюших разрезов. Таким образом, аналитическим дугам $S_{j}$, составляющим компакт минимальной емкости, соответствуют на $\mathfrak{R}$ замкнутые аналитические (в комплексной структуре $\mathfrak{R}$ ) жордановы кривые $\Gamma_{j}, j=1, \ldots, \mathrm{g}+1,-$ циклы на $\mathfrak{R} ;$ положим $\Gamma=\bigcup_{j=1}^{\mathrm{g}+1} \Gamma_{j}$. Листы римановой поверхности будем обозначать соответственно через $D^{(1)}$ и $D^{(2)}$; имеем $\Re=D^{(1)} \cup \Gamma \cup D^{(2)}$, каждьй из листов - g-связная область, первый лист $D^{(1)}$ мы будем отождествлять с многосвязной областью $D=\overline{\mathbb{C}} \backslash S$. Для точек $\mathbf{z}$, лежаших на первом листе, $\mathbf{z} \in D^{(1)}$, имеем $\mathbf{z}=(z, \sqrt{h(z)})$, где, как и вьше, выбрана однозначная в $D$ ветвь $\sqrt{h(z)}$, удовлетворяющая условию $\sqrt{h(z)} / z^{\mathrm{g}+1} \rightarrow 1$ при $z \rightarrow \infty$; для $\mathbf{z} \in D^{(2)}$ имеем: $\mathbf{z}=(z,-\sqrt{h(z)})$; для явного указания, на каком именно листе римановой поверхности лежит точка $\mathbf{z}$, мы будем часто использовать сокрашенную запись $z^{(1)}$ при $\mathbf{z} \in D^{(1)}$ и $z^{(2)}$ при $\mathbf{z} \in D^{(2)}$, причем в соответствии с нашим соглашением об отождествлении $D$ и $D^{(1)}$ будем считать, что $z=z^{(1)}$. Соответственно $w(\mathbf{z})=\sqrt{h(z)}$ при $\mathbf{z}=z^{(1)}$ и $w(\mathbf{z})=-\sqrt{h(z)}$ при $\mathbf{z}=z^{(2)}$. Для $\mathbf{z}=z^{(1)}$ будем часто писать просто $w(z)$ вместо $w(\mathbf{z})$; тем самьм, приобретает смысл и запись $w^{+}(\zeta), \zeta \in S$. Каноническая проекция $\boldsymbol{\pi}: \mathfrak{R} \rightarrow \overline{\mathbb{C}}$ определяется соотношением $\boldsymbol{\pi}(\mathbf{z})=z$, в частности $\boldsymbol{\pi}\left(D^{(1)}\right)=\boldsymbol{\pi}\left(D^{(2)}\right)=D$. Две различные точки $\mathbf{z}$ и $\boldsymbol{\zeta}$ будем назьвать (взаимно) сопряженными и писать $\boldsymbol{\zeta}=\mathbf{z}^{*}$, если $\boldsymbol{\pi}(\mathbf{z})=\boldsymbol{\pi}(\boldsymbol{\zeta})$. Напомним, что $\boldsymbol{\pi}(\Gamma)=S$.

Положительньм обходом контура $\Gamma$ на $\Re$ будем считать такой, при котором область $D^{(1)}$ остается слева, а область $D^{(2)}$ - справа; соответствующее обозначение $\Gamma^{+}$. Предельное положение точки $\mathbf{z} \in D^{(1)}$ (соответственно $\left.\mathbf{z} \in D^{(2)}\right)$ на $\Gamma$, когда $\mathbf{z}$ стремится к точке $\boldsymbol{\zeta} \in \Gamma$, будем обозначать $\boldsymbol{\zeta}+($ соответственно $\boldsymbol{\zeta}-)$. Если в $D^{(1)}$ задана функция $F(\mathbf{z})$, то под $F(\boldsymbol{\zeta}+)$ понимаются ее радиальные (в комплексной структуре $\mathfrak{R}$ ) предельные значения, если они сушествуют, в точке $\zeta \in \Gamma$; аналогичный смысл придается и $F(\boldsymbol{\zeta}-)$.

2.2. Первые g из контуров $\Gamma_{j}$ выберем в качестве базисных $a$-циклов $\left\{\mathbf{a}_{j}, j=1\right.$, $\ldots, \mathrm{g}\}$, а затем стандартным образом дополним этот набор $b$-циклами $\left\{\mathbf{b}_{j}, j=1\right.$, $\ldots, \mathrm{g}\}$ так, что матрища пересечений имеет вид $\mathbf{a}_{k} \circ \mathbf{a}_{j}=\mathbf{b}_{k} \circ \mathbf{b}_{j}=0, j \neq k, \mathbf{a}_{k} \circ \mathbf{b}_{j}=$ $\delta_{k j}$ и циклы $\left\{\mathbf{a}_{j}, \mathbf{b}_{j}\right\}$ образуют базис гомологий на $\mathfrak{R}$, т.е. любой цикл с на $\mathfrak{R}$ гомологичен целочисленной линейной комбинации $\mathbf{a}_{j}$ и $\mathbf{b}_{j}: \mathbf{c}=\sum_{j=1}^{\mathrm{g}} n_{j} \mathbf{a}_{j}+m_{j} \mathbf{b}_{j}, n_{j}, m_{j} \in \mathbb{Z}$. Для произвольного голоморфного абелева дифференциала $d \Omega$ функция $\int^{\mathbf{z}} d \Omega$ определяется однозначно по модулю его $a$ - и $b$-периодов: $\oint_{\mathbf{a}_{j}} d \Omega$ и $\oint_{\mathbf{b}_{j}} d \Omega$. Как известно, введенный вьше канонический базис гомологий $\left\{\mathbf{a}_{j}, \mathbf{b}_{j}\right\}$ порождает на $\Re$ соответствующий а-нормированный базис голоморфных абелевых дифференциалов $\left\{d \Omega_{k}\right\}$ : 
$\oint_{\mathbf{a}_{j}} d \Omega_{k}(\boldsymbol{\zeta})=\delta_{k j}, k, j=1, \ldots$, g. Для такого базиса матрища $\mathbf{B}=\left\|B_{k j}\right\|_{k, j=1, \ldots, \mathrm{g}}$, $B_{k j}=\oint_{\mathbf{b}_{j}} d \Omega_{k}(\boldsymbol{\zeta})$, является матрицей Римана: она симметрична, $B_{k j}=B_{j k}$, а ее мнимая часть положительно определена, $\left\|\operatorname{Im} B_{j k}\right\|>0$.

Фиксируем стандартный базис $\mathbf{e}_{1}, \mathbf{e}_{2}, \ldots, \mathbf{e}_{\mathbf{g}}$ в $\mathbb{R}^{\mathrm{g}}:\left(\mathbf{e}_{k}\right)_{j}=\delta_{k j}$. Тогда векторы $\mathbf{e}_{1}, \ldots, \mathbf{e}_{\mathrm{g}}, \mathbf{B e}_{1}, \ldots, \mathbf{B e}_{\mathrm{g}}$ линейно независимы над $\mathbb{R}$ и образуют базис в $\mathbb{C}^{\mathrm{g}}$. Факторпространство $\mathbb{C}^{\mathrm{g}} /\{\mathrm{N}+\mathrm{BM}\}$ по целочисленной решетке, $\mathrm{N}, \mathrm{M} \in \mathbb{Z}^{\mathrm{g}}$, является $2 \mathrm{~g}$-мерным вешественным тором $\mathbb{T}^{2 \mathrm{~g}}$ и называется многообразием Якоби Jас $\mathfrak{R}$ римановой поверхности $\mathfrak{R}$. Любой вектор $\mathbf{v} \in \mathbb{C}^{\mathrm{g}}$ однозначно представим в виде $\mathbf{v}=\mathbf{x}+\mathbf{B y}+$ $\mathrm{N}+\mathbf{B M}=\mathbf{x}+\mathbf{B y}(\bmod$ периодов $), 0 \leqslant(\mathbf{x})_{j},(\mathbf{y})_{j}<1, \mathrm{~N}, \mathrm{M} \in \mathbb{Z}^{\mathbf{g}} ;$ иногда для краткости знак сравнения по модулю периодов дифференциалов $\left\{d \Omega_{k}\right\}$ мы будем опускать и писать просто $\mathbf{v} \equiv \mathbf{x}+\mathbf{B y}$. Вешественные числа $x_{j}=(\mathbf{x})_{j}, y_{j}=(\mathbf{y})_{j} \in[0,1)$, $j=1, \ldots, \mathrm{g}$, будем называть соответственно $x$ - и $y$-координатами вектора $\mathbf{v}$ в многообразии Якоби (точнее, класса $[\mathbf{v}]$ всех эквивалентных $\mathbf{v}$ векторов). Пусть $\mathbf{z}_{0}-$ некоторая фиксированная точка римановой поверхности $\mathfrak{R}$. Вектор-функция $A(\mathbf{z})=$ $\left(A_{1}(\mathbf{z}), \ldots, A_{\mathbf{g}}(\mathbf{z})\right)$ с координатами

$$
A_{j}(\mathbf{z})=\int_{\mathbf{z}_{0}}^{\mathbf{z}} d \Omega_{j} \quad(\bmod \text { периодов }), \quad j=1, \ldots, \mathrm{g},
$$

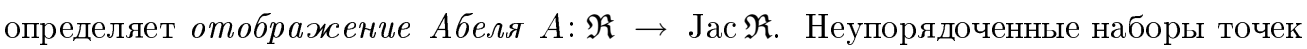
$\mathbf{z}_{1}, \ldots, \mathbf{z}_{\mathbf{g}}, \mathbf{z}_{j} \in \mathfrak{R}$, образуют g-ю симметрическую степень $S^{\mathrm{g}} \mathfrak{R}$ римановой поверхности $\mathfrak{R}$. Вектор-функция $\mathfrak{A}\left(\mathbf{z}_{1}, \ldots, \mathbf{z}_{\mathrm{g}}\right)=\left(\mathfrak{A}_{1}, \ldots, \mathfrak{A}_{\mathrm{g}}\right)$ с координатами

$$
\mathfrak{A}_{k}=\mathfrak{A}_{k}\left(\mathbf{z}_{1}, \ldots, \mathbf{z}_{\mathrm{g}}\right) \equiv \sum_{j=1}^{\mathrm{g}} A_{k}\left(\mathbf{z}_{j}\right) \equiv \sum_{j=1}^{\mathrm{g}} \int_{\mathbf{z}_{0}}^{\mathbf{z}_{j}} d \Omega_{k}, \quad k=1, \ldots, \mathrm{g}
$$

также назьвается отображсением Абеля $\mathfrak{A}: S^{\mathrm{g}} \mathfrak{R} \rightarrow$ Јас $\mathfrak{R}$. Если задан вектор $\mathbf{v}=$ $\left(v_{1}, \ldots, v_{\mathbf{g}}\right) \in \mathrm{Jac} \mathfrak{R}$, то задача нахождения неупорядоченного набора точек $\mathbf{z}_{1}, \ldots, \mathbf{z}_{\mathfrak{g}}$, $\mathbf{z}_{j} \in \Re$, для которого

$$
\mathfrak{A}_{k}\left(\mathbf{z}_{1}, \ldots, \mathbf{z}_{\mathrm{g}}\right)=v_{k} \quad(\bmod \text { периодов }), \quad k=1, \ldots, \mathrm{g}
$$

называется проблемой Якоби обрашения абелевых интегралов или просто проблемой обращения Якоби. Хорошо известно, что проблема обрашения Якоби всегда разрешима, но, вообше говоря, не единственным образом.

2.3. Для любых двух различных точек $\mathbf{z}_{1}$ и $\mathbf{z}_{2}$ римановой поверхности $\mathfrak{R}$ сушествует абелев дифференциал третьего рода, голоморфный всюду на $\mathfrak{R}$, за исключением этих двух точек, в которых он имеет простые полюсы с вычетами соответственно 1 и -1 ; такой дифференциал называется нормальныцм. Нормальный дифференциал будет определен однозначно, если потребовать, чтобы все его $a$-периоды были равны нулю; будем назьвать такой дифференциал $а$-нормированным абелевым дифференциалом третьего рода и обозначать $d \Omega\left(\mathbf{z}_{1}, \mathbf{z}_{2} ; \mathbf{z}\right)$; для $a$-нормированного дифференциала $d \Omega\left(\infty^{(1)}, \infty^{(2)} ; \mathbf{z}\right)$ будем иногда для краткости использовать обозначение $d \Omega(\mathbf{z})$. 
Другой способ однозначно задать (нормировать) дифференциал третьего рода - потребовать, чтобы все его периоды были чисто мнимьми. Таким, например, является дифференщиал комплексной функции Грина $G_{D}(z, \infty)=g_{D}(z, \infty)+i \widetilde{g}_{D}(z, \infty)$ (здесь $\widetilde{g}_{D}(z, \infty)$ - функция, гармонически сопряженная к функции Грина $\left.g_{D}(z, \infty)\right)$, соответствуюшей компакту минимальной емкости $S$. Хорошо известно [35], [39], что

$$
G_{D}(z, \infty)=\int_{e_{1}}^{z} \frac{p_{\mathrm{g}}(\zeta)}{\sqrt{h_{2 \mathrm{~g}+2}(\zeta)}} d \zeta, \quad z \in D=\overline{\mathbb{C}} \backslash S,
$$

где $p_{\mathrm{g}}(z)=p_{\mathrm{g}}(z ; h)=z^{\mathrm{g}}+\cdots \in \mathbb{C}[z]-$ некоторый полином, нули которого при условии $\left(C_{1}\right)$ лежат вне $S$. Дифференциал $d G(\mathbf{z})=d G\left(\infty^{(1)}, \infty^{(2)} ; \mathbf{z}\right)=p_{\mathbf{g}}(z) d z / w(\mathbf{z})$ определен на всей римановой поверхности $\mathfrak{R}$, имеет чисто мнимые периоды и простые полюсы в точках $\mathbf{z}=\infty^{(1)}$ и $\mathbf{z}=\infty^{(2)}$ с вычетами соответственно -1 и 1 . Следовательно, функция $u(\mathbf{z})=\operatorname{Re} \int_{e_{1}}^{\mathbf{z}} d G(\boldsymbol{\zeta})$ - однозначная гармоническая функция на $\mathfrak{R} \backslash\left\{\infty^{(1)}, \infty^{(2)}\right\}$, линия уровня которой $\{\mathbf{z}: u(\mathbf{z})=0\}$ совпадает с $\Gamma$, а $g_{D}(z, \infty)=$ $u\left(z^{(1)}\right), z \in D$. Важную роль в наших рассуждениях играет следующее соотношение между дифференщиалом $d G\left(\infty^{(1)}, \infty^{(2)} ; \mathbf{z}\right)$ и $a$-нормированньмм дифференщиалом $d \Omega\left(\infty^{(1)}, \infty^{(2)} ; \mathbf{z}\right)$ :

$$
d G\left(\infty^{(1)}, \infty^{(2)} ; \mathbf{z}\right)+2 \pi i \sum_{k=1}^{\mathrm{g}} \omega_{k}(\infty) d \Omega_{k}(\mathbf{z})+d \Omega\left(\infty^{(1)}, \infty^{(2)} ; \mathbf{z}\right)=0
$$

здесь $\omega_{k}(\infty), k=1, \ldots, \mathrm{g},-$ гармоническая мера $k$-й компоненты $S_{k}$ относительно $D$ в бесконечно удаленной точке (доказательство см. в $\S 4$ ).

Как известно, $a$-периоды дифференциала комплексной функции Грина связаны с гармоническими мерами $\omega_{j}(\infty)$ следующим образом:

$$
\oint_{\mathbf{a}_{k}} d G\left(\infty^{(1)}, \infty^{(2)} ; \mathbf{z}\right)=-2 \pi i \omega_{k}(\infty)
$$

Положим по аналогии

$$
\Delta_{k}(\infty):=\frac{1}{2 \pi i} \oint_{\mathbf{b}_{k}} d G\left(\infty^{(1)}, \infty^{(2)} ; \mathbf{z}\right) \in \mathbb{R} .
$$

Подобным образом, если для произвольной точки $z_{0} \in \overline{\mathbb{C}} \backslash\left\{e_{1}, \ldots, e_{2 \mathrm{~g}+2}\right\}$ через $S\left(z_{0}\right)$ обозначим множество минимальной емкости относительно этой точки, содержашее $e_{1}, \ldots, e_{2 g+2}$, то при условии, что кривые $\Gamma_{k}\left(z_{0}\right)$ гомотопны кривым $\Gamma_{k}, k=$ $1, \ldots, \mathrm{g}+1$, для дифференщиала $d G\left(z_{0}^{(1)}, z_{0}^{(2)} ; \mathbf{z}\right)$, соответствующего комплексной функции Грина $G_{\overline{\mathbb{C}} \backslash S\left(z_{0}\right)}\left(z, z_{0}\right)$, имеем

$$
d G\left(z_{0}^{(1)}, z_{0}^{(2)} ; \mathbf{z}\right)+2 \pi i \sum_{k=1}^{\mathrm{g}} \omega_{k}\left(z_{0}\right) d \Omega_{k}(\mathbf{z})+d \Omega\left(z_{0}^{(1)}, z_{0}^{(2)} ; \mathbf{z}\right)=0,
$$


где $\omega_{k}\left(z_{0}\right)=\omega_{k}\left(z_{0} ; S\left(z_{0}\right)\right), k=1, \ldots, \mathrm{g},-$ соответствующая гармоническая мера $k$-й компоненты. Последнее соотношение понадобится нам в дальнейшем только для случая, когда все точки $e_{1}, \ldots, e_{2 \mathrm{~g}+2}$ лежат на одной прямой и, тем самым, множество минимальной емкости $S$ состоит из отрезков $\left[e_{2 j-1}, e_{2 j}\right], j=1, \ldots, \mathrm{g}+1$, а $S\left(z_{0}\right)=S$ для любой точки $z_{0}$, лежащей в лакуне между этими отрезками, в том числе - содержашей бесконечно удаленную точку.

Приведем также используемые в дальнейшем "явные" представления для некоторых $a$-нормированных абелевых дифференциалов третьего рода:

$$
\begin{gathered}
d \Omega\left(z_{1}^{(1)}, z_{2}^{(1)} ; \mathbf{z}\right)=\frac{1}{2}\left\{\frac{w(\mathbf{z})+w\left(\mathbf{z}_{1}\right)}{z-z_{1}} \frac{d z}{w(\mathbf{z})}-\frac{w(\mathbf{z})+w\left(\mathbf{z}_{2}\right)}{z-z_{2}} \frac{d z}{w(\mathbf{z})}+\frac{p_{1}(z)}{w(\mathbf{z})} d z\right\} \\
d \Omega\left(z_{1}^{(1)}, \infty^{(2)} ; \mathbf{z}\right)=\frac{1}{2} \frac{w(\mathbf{z})+w\left(\mathbf{z}_{1}\right)}{z-z_{1}} \frac{d z}{w(\mathbf{z})}-\frac{1}{2} \frac{z^{\mathrm{g}}+p_{2}(z)}{w(\mathbf{z})} d z \\
d \Omega\left(\infty^{(1)}, \infty^{(2)} ; \mathbf{z}\right)=-\frac{z^{\mathrm{g}}+p_{3}(z)}{w(\mathbf{z})} d z
\end{gathered}
$$

$p_{j} \in \mathbb{C}[z], \operatorname{deg} p_{j} \leqslant \mathrm{~g}-1$.

Рассеченная риманова поверхность $\widetilde{\mathfrak{R}}$ получается из $\mathfrak{R}$ проведением сечений по базисным циклам $\mathbf{a}_{k}, \mathbf{b}_{k}, k=1, \ldots, \mathrm{g}$, поверхности: $\widetilde{\Re}=\mathfrak{R} \backslash\left(\bigcup_{k=1}^{g} \mathbf{a}_{k} \cup \bigcup_{k=1}^{\mathrm{g}} \mathbf{b}_{k}\right)$; $\widetilde{\Re}$ связна (переход с одного листа на другой осуществляется через $\Gamma_{\mathrm{g}+1}$ ) и односвязна (она эквивалентна 4g-угольнику). Для $b$-периодов $a$-нормированного абелева дифференциала $d \Omega\left(\mathbf{z}_{1}, \mathbf{z}_{2} ; \mathbf{z}\right)$ справедливо следуюшее соотношение Римана:

$$
\int_{\mathbf{b}_{j}} d \Omega\left(\mathbf{z}_{1}, \mathbf{z}_{2} ; \zeta\right)=-2 \pi i \int_{\mathbf{z}_{1}}^{\mathbf{z}_{2}} d \Omega_{j}(\boldsymbol{\zeta}), \quad j=1, \ldots, \mathrm{g},
$$

где интеграл берется по произвольному пути, соединяющему точки $\mathbf{z}_{1}$ и $\mathbf{z}_{2}$ и лежащему в $\widetilde{\Re}$.

2.4. Дивизором $d$ на римановой поверхности $\Re$ будем назьвать формальный символ $d=\mathbf{z}_{1}^{\nu_{1}} \mathbf{z}_{2}^{\nu_{2}} \cdots \mathbf{z}_{m}^{\nu_{m}}$, где точки $\mathbf{z}_{j} \in \mathfrak{R}, \nu_{j} \in \mathbb{Z} ;|d|=\sum_{j=1}^{m} \nu_{j}$ - степень дивизора $d$; если точки $\mathbf{z}_{j}$ попарно различны и все $\nu_{j} \geqslant 0$, то дивизор $d-$ чельй. Произвольной функции $F$, мероморфной на $\Re, F \in \mathbb{C}(z, w)$, сопоставим дивизор $(F)$ ее нулей и полюсов: $(F)=\mathbf{z}_{1}^{\nu_{1}} \cdots \mathbf{z}_{\ell}^{\nu_{\ell}} \cdot \zeta_{1}^{-\mu_{1}} \cdots \zeta_{m}^{-\mu_{m}}$, где $\mathbf{z}_{j}, \boldsymbol{\zeta}_{j} \in \mathfrak{R}-$ соответственно нули и полюсы $F$, а $\nu_{j}, \mu_{j} \geqslant 0$ - их кратности; такие дивизоры называются главнылми. По теореме Абеля дивизор $d=\mathbf{z}_{1}^{\nu_{1}} \cdots \mathbf{z}_{\ell}^{\nu_{\ell}} \cdot \zeta_{1}^{-\mu_{1}} \cdots \zeta_{m}^{-\mu_{m}}$ - главньй тогда и только тогда, когда $|d|=0$ и для всех $k=1, \ldots, \mathrm{g}$

$$
\left\{\sum_{j=1}^{\ell} \nu_{j} \int_{\infty^{(1)}}^{\mathbf{z}_{j}}-\sum_{j=1}^{m} \mu_{j} \int_{\infty^{(1)}}^{\boldsymbol{\zeta}_{j}}\right\} d \Omega_{k}=0 \quad(\bmod \text { периодов }) .
$$

Два дивизора $d_{1}$ и $d_{2}$ эквивалентнь, если их отношение $d_{1} / d_{2}-$ главный дивизор. Хорошо известно (см., например, [69], а также [64], [35]), что два цельх дивизора $d_{1}=\mathbf{z}_{1} \cdots \mathbf{z}_{\mathrm{g}}$ и $d_{2}=\zeta_{1} \cdots \zeta_{\mathrm{g}}$ степени $\mathrm{g}$, заданных на гиперэллиптической римановой поверхности рода $\mathrm{g}$, эквивалентны тогда и только тогда, когда

$$
d_{1}=\mathbf{t}_{1} \mathbf{t}_{1}^{*} \cdots \mathbf{t}_{2 m} \mathbf{t}_{2 m}^{*} \mathbf{z}_{2 m+1} \cdots \mathbf{z}_{\mathrm{g}}, \quad d_{2}=\tau_{1} \tau_{1}^{*} \cdots \tau_{2 m} \tau_{2 m}^{*} \mathbf{z}_{2 m+1} \cdots \mathbf{z}_{\mathrm{g}},
$$


$m \leqslant \mathrm{~g} / 2$. Отсюда непосредственно вытекает следующее свойство отображения Абеля $\mathfrak{A}: S^{\mathrm{g}} \mathfrak{R} \rightarrow$ Јас $\mathfrak{R}$. Пусть $\mathscr{U} \subset S^{\mathrm{g}} \mathfrak{R}-$ произвольное множество, устроенное следующим образом: $\mathscr{U}$ представляет собой (неупорядоченное) произведение g непересекающихся компактов $\mathscr{U}_{j}$ в $D^{(1)}, \mathscr{U}_{j} \cap \mathscr{U}_{k}=\varnothing$ при $j \neq k$. Тогда $\mathfrak{A}^{-1}(\mathfrak{A}(\mathscr{U}))=\mathscr{U}$.

2.5. Предположим, что на $\mathfrak{R} \backslash \Gamma$ задана (кусочно-) мероморфная функция $F(\mathbf{z})$, имеюшая полюсы только в бесконечно удаленных точках $\mathbf{z}=\infty^{(1)}$ и $\mathbf{z}=\infty^{(2)}$, каждьй порядка $\leqslant \mathrm{g}$, предельные значения которой на Г сушествуют и удовлетворяют условию:

$$
F(\boldsymbol{\zeta}+)-F(\zeta-)=\chi(\zeta), \quad \zeta \in \Gamma,
$$

где $\chi$ - Н-непрерьвна на Г. Тогда с помошњю мероморфного абелева дифференциала

$$
d \Omega(\mathbf{z} ; \zeta)=\frac{1}{2} \frac{w(\boldsymbol{\zeta})+w(\mathbf{z})}{\zeta-z} \frac{d \zeta}{w(\zeta)}
$$

заданного на римановой поверхности $\mathfrak{R}$, функщия $F(\mathbf{z})$ восстанавливается по граничным условиям (2.11) интегралом типа Коши:

$$
F(\mathbf{z})=-\frac{1}{2 \pi i} \int_{\Gamma^{+}} \chi(\boldsymbol{\zeta}) d \Omega(\mathbf{z} ; \boldsymbol{\zeta})+p(z), \quad \mathbf{z} \notin \Gamma
$$

где $p(z) \in \mathbb{C}[z], \operatorname{deg} p \leqslant \mathrm{~g}$, а контур $\Gamma^{+}$ориентирован положительно относительно области $D^{(1)}$ : область остается слева при обходе по контуру.

Действительно, легко проверяются следуюшие свойства дифференщиала (2.12):

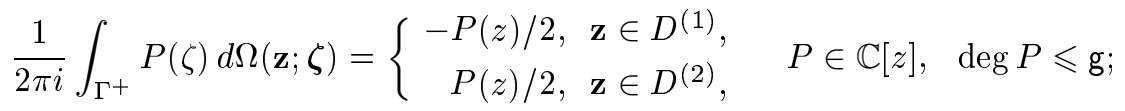

дифференщиал $d \Omega(\mathbf{z} ; \zeta)$ имеет полюсы только в точках $\mathbf{z}, \infty^{(1)}, \infty^{(2)}$ с вычетами соответственно $1,-1 / 2,-1 / 2$.

Если функция $\chi$ Н-непрерьвна на $\Gamma$, то функция

$$
F_{\chi}(\mathbf{z})=-\frac{1}{2 \pi i} \int_{\Gamma^{+}} \chi(\boldsymbol{\zeta}) d \Omega(\mathbf{z} ; \boldsymbol{\zeta}), \quad \mathbf{z} \notin \Gamma,
$$

обладает следующими свойствами:

1) $F_{\chi}$ голоморфна в $D^{(1)} \backslash\left\{\infty^{(1)}\right\}$ и $D^{(2)} \backslash\left\{\infty^{(2)}\right\}$;

2) $F_{\chi}(\mathbf{z})=O\left(z^{\mathbf{g}}\right)$ при $\mathbf{z} \rightarrow \infty^{(1)}$ или $\mathbf{z} \rightarrow \infty^{(2)}$;

3) справедлива формула Сохоцкого

$$
F_{\chi}\left(\boldsymbol{\zeta}_{0} \pm\right)= \pm \frac{1}{2} \chi\left(\boldsymbol{\zeta}_{0}\right)-\frac{1}{2 \pi i} \int_{\Gamma^{+}} \chi(\boldsymbol{\zeta}) d \Omega\left(\boldsymbol{\zeta}_{0} ; \boldsymbol{\zeta}\right)
$$

(последний интеграл понимается как особьй);

4) $F_{\chi}\left(\zeta_{0}+\right)-F_{\chi}\left(\zeta_{0}-\right)=\chi\left(\zeta_{0}\right), \zeta_{0} \in \Gamma$. 
Рассмотрим разность $v(\mathbf{z})=F(\mathbf{z})-F_{\chi}(\mathbf{z})$. В силу (2.11) эта функция продолжается на всю $\mathfrak{R}$ как мероморфная функция, т.е. $v \in \mathbb{C}(z, w)$, а следовательно, имеет вид $v(\mathbf{z})=$ $r_{1}(z)+r_{2}(z) w(\mathbf{z}), r_{1}, r_{2} \in \mathbb{C}(z)$. Так как $v$ может иметь полюсы порядка $\leqslant$ g и только в точках $\infty^{(1)}$ и $\infty^{(2)}$, то $r_{j}=p_{j} \in \mathbb{C}[z]$. Следовательно, $v(\mathbf{z})=p_{1}(z)+p_{2}(z) w(\mathbf{z})$. Учитьвая, что $|w(\mathbf{z})| \sim|z|^{\mathbf{g}+1}$ при $\mathbf{z} \rightarrow \infty^{(1)}, \infty^{(2)}$, получаем $p_{2} \equiv 0, \operatorname{deg} p_{1} \leqslant \mathrm{~g}$. Формула (2.13) доказана.

В заключение приведем некоторые “явные” представления дифференциала (2.12). При $\zeta=\zeta^{(1)}, \mathbf{z}=z^{(1)}$ или $\zeta=\zeta^{(2)}, \mathbf{z}=z^{(2)}$ имеем

$$
d \Omega\left(z^{(1)} ; \zeta^{(1)}\right)=d \Omega\left(z^{(2)} ; \zeta^{(2)}\right)=\frac{1}{2} \frac{\sqrt{h(\zeta)}+\sqrt{h(z)}}{\zeta-z} \frac{d \zeta}{\sqrt{h(\zeta)}}
$$

при $\zeta=\zeta^{(2)}, \mathbf{z}=z^{(1)}$ или $\zeta=\zeta^{(1)}, \mathbf{z}=z^{(2)}$ имеем

$$
d \Omega\left(z^{(1)} ; \zeta^{(2)}\right)=\frac{1}{2} \frac{\sqrt{h(\zeta)}-\sqrt{h(z)}}{\zeta-z} \frac{d \zeta}{\sqrt{h(\zeta)}}
$$

т.е. в этом случае при $\zeta=z$ дифференциал особенности не имеет.

\section{§. Краевая задача Римана}

В этом параграфе мы исследуем сформулированную во введении краевую задачу Римана. Нетрудно видеть, что с учетом сделанных выше обозначений задача (R) эквивалентна следуюшей краевой задаче Римана на двулистной гиперэллиптической поверхности $\mathfrak{R}$.

ЗАДАчА $\left(\mathscr{R}^{\prime}\right)$. Найти функцию $\Psi(\mathbf{z})$, мероморфную в $\mathfrak{R} \backslash \Gamma$ и такую, что

(i) дивизор функции $\Psi$ имеет вид: $(\Psi)=\left(\infty^{(2)}\right)^{n-\mathrm{g}} \cdot z_{1, n}^{(2)} \cdots z_{\mathrm{g}, n}^{(2)} /\left(\infty^{(1)}\right)^{n}$;

(ii) на кривой $\Gamma$ выполняется следующее краевое условие: $\rho(\zeta) \Psi(\zeta+)=\Psi(\zeta-)$, $\zeta \in \Gamma$.

Действительно, положим $\varphi_{n}(z)=\Psi\left(z^{(1)}\right), \psi_{n}(z)=\Psi\left(z^{(2)}\right)$. Теперь доказательство эквивалентности двух указанных задач не составляет труда, если учесть, что множество $S$ - каноническая проекция Г на $\overline{\mathbb{C}}$.

Благодаря эквивалентности задач $(\mathscr{R})$ и $\left(\mathscr{R}^{\prime}\right)$ анализ сушествования решения задачи $(\mathscr{R})$ оказьвается возможньм провести на основе исследования проблемы обрашения Якоби. А именно, имеет место

ОСНОВНАЯ ЛЕММА. При фиксированном $n \in \mathbb{N}$ справедливь следующие утверждения.

(a) Если решение задачи $(\mathscr{R})$ существует, то оно единственно.

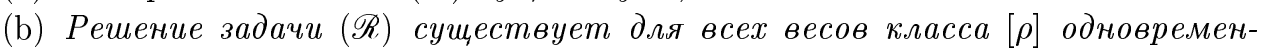
но. Для существования решения необходимо и достаточно, чтобы проблема обращения Якоби

$$
\begin{gathered}
\sum_{j=1}^{\mathrm{g}} \int_{\infty}^{\mathbf{z}_{j, n}} d \Omega_{k}(\zeta) \equiv \frac{i}{\pi} \int_{S} \log \rho(\zeta) d \Omega_{k}^{+}(\zeta)+\left\{n \Delta_{k}(\infty)\right\}+\sum_{j=1}^{\mathrm{g}}\left\{n \omega_{j}(\infty)\right\} B_{k j} \\
k=1, \ldots, \mathrm{g}
\end{gathered}
$$


имела решение, принадлежащее первому листу: $\mathbf{z}_{j, n}=z_{j, n}^{(1)}, j=1, \ldots, \mathrm{g}$ (здесь $\{\cdot\}$ - дробная часть соответствуюшего числа).

(c) Справедливо следующее "явное" представление решения $\varphi_{n}, \psi_{n}$ задачи (R):

$$
\varphi_{n}(z ; \rho)=\operatorname{cap}(S)^{n} \Phi(z)^{n} F_{n}(z ; \rho), \quad \psi_{n}(z ; \rho)=\operatorname{cap}(S)^{n} \frac{X_{\mathrm{g}, n}(z)}{\Phi(z)^{n} F_{n}(z ; \rho)}
$$

где $\Phi(z)$ - отображсоющая функиия, $X_{\mathrm{g}, n}(z)=\prod_{j=1}^{\mathrm{g}}\left(z-z_{j, n}\right), F_{n}(z ; \rho)$ - многозначная аналитическая в области $D$ функиия, модуль которой однозначен $u$ $\left|F_{n}(\infty ; \rho)\right|=1 ; F_{n}$ зависит от класса әквивалентности $[\rho]$, а ее модуль равномерно по $n \in \Lambda$ ограничен и отграничен от нуля внутри области $D$.

В дальнейшем (см. $\S 4)$ мы докажем сушествование решения проблемы обращения Якоби (2.15), удовлетворяюшего условиям п. (b), по некоторой подпоследовательности $\Lambda \subset \mathbb{N}$ в двух случаях: (I) в ситуации, когда все точки $e_{1}, \ldots, e_{2 \mathrm{~g}+2}$ лежат на одной прямой, а гармонические меры $\omega_{1}(\infty), \ldots, \omega_{\mathrm{g}+1}(\infty)$ рационально независимы (как уже отмечалось, если все точки $\left\{e_{j}\right\}$ лежат на одной прямой, то в “общем положении" это условие на гармонические меры отрезков всегда вьполняется), и (II) в случае "общего положения" $\left(C_{1}\right)-\left(C_{2}\right)$. Тем самым для этой подпоследовательности $\Lambda$ будут доказаны и утверждения п. (c).

Более того, в этих двух случаях (I) и (II) можно доказать, что сушествует решение некоторой “модифицированной” проблемы обрашения Якоби (подробнее см. ниже § 4), откуда уже легко получаем следуюшее

СлЕДСТВИЕ. Пусть в области $D^{*}=D \backslash\{\infty\}$ задана последовательность дивизоров $d_{n}=a_{1} \cdots a_{m} /\left(a_{1, n} \cdots a_{m, n}\right)$ нулевой степени таких, что все $a_{j, n} \rightarrow$ $a_{j} \in D^{*}$ при $n \rightarrow \infty, j=1, \ldots, m$. Тогда в условиях "общего положсения" (I) или (II) существует подпоследовательность $\Lambda \subset \mathbb{N}$ такая, что для всех $n \in \Lambda$ найдется пара функиий $\varphi_{n}, \psi_{n} \in \mathscr{C}(D)$ таких, что

(i) $\varphi_{n}$ имеет полюс порядка $n$ в точке $z=\infty$ инуль в каждой точке $a_{j}$, $j=1, \ldots, m$ (т.е. дивизор функции $\varphi_{n}$ имеет вид: $\left.\left(\varphi_{n}\right)_{D}=\infty^{-n} \cdot a_{1} \cdots a_{m}\right)$;

(ii) $\psi_{n}$ имеет нуль кратности $n-\mathrm{g}$ в точке $z=\infty$, полюс в каждой точке $a_{j, n}, j=1, \ldots, m$, и еще ровно g “свободньх" нулей, все предельные точки которых принадлежат области $D^{*}$ (т.е. дивизор функции $\psi_{n}$ имеет вид: $\left(\psi_{n}\right)_{D}=\infty^{n-\mathrm{g}} \cdot z_{1, n} \cdots z_{\mathrm{g}, n} \cdot a_{1, n}^{-1} \cdots a_{m, n}^{-1}$, все $\left.z_{j, n} \in D^{*}\right) ;$

(iii) на множестве $S$ выполняются краевые условия $\rho \varphi_{n}^{+}=\psi_{n}^{-}, \rho \varphi_{n}^{-}=\psi_{n}^{+}$.

При этом для функиий $\varphi_{n}, \psi_{n}$ справедливь представления (2.16), в которых точки $z_{1, n}, \ldots, z_{\mathrm{g}, n}$ являются решениями некоторой "возмущенной" проблемь обращения Якоби, ак функиии $F_{n}(z)$ добавлень соответственно сомножители вида

$$
\text { const } \exp \left\{\sum_{j=1}^{m} \Omega\left(a_{j}^{(1)}, \infty^{(2)} ; z^{(1)}\right)\right\} \quad u \quad \text { const } \exp \left\{-\sum_{j=1}^{m} \Omega\left(a_{j, n}^{(2)}, \infty^{(2)} ; z^{(1)}\right)\right\}
$$

сохраняющие ее основные свойства. 
ДокАЗАТЕЛЬСТво основноЙ лЕммЫ. (а) Единственность. Для доказательства единственности решения задачи $(\mathscr{R})$ воспользуемся эквивалентностью $(\mathscr{R})$ и $\left(\mathscr{R}^{\prime}\right)$. Предположим, что существуют два решения задачи $(\mathscr{R}):(\varphi, \psi)$ и $(\widetilde{\varphi}, \widetilde{\psi})$ (здесь и далее в этом пункте зависимость от $n$ мы явно не указываем). Тогда функция $F\left(z^{(2)}\right)=$ $\psi(z) / \widetilde{\psi}(z), z^{(2)} \in D^{(2)}, F\left(z^{(1)}\right)=\varphi(z) / \widetilde{\varphi}(z), z^{(1)} \in D^{(1)}$, “склеивается" в единую мероморфную функцию $F(\mathbf{z})$, заданную на всей поверхности $\mathfrak{R}$, дивизор которой имеет вид $(F)=z_{1}^{(2)} \cdots z_{\mathrm{g}}^{(2)} /\left(\widetilde{z}_{1}^{(2)} \cdots \widetilde{z}_{\mathrm{g}}^{(2)}\right)$. Из упомянутого в п. 2.4 свойства эквивалентных дивизоров, заданных на гиперэллиптической римановой поверхности, вытекает, что после надлежащей перенумерации все $z_{j}^{(2)}=\widetilde{z}_{j}^{(2)}$. Следовательно, $(F)=1$ и $F \equiv$ const.

(b) Необходимость. Пусть пара функций $\varphi, \psi$ - решение задачи $(\mathscr{R}), z_{1}, \ldots, z_{\mathrm{g}}-$ свободные нули функции $\psi$. Перемножая краевые условия $\rho \varphi_{n}^{+}=\psi_{n}^{-}, \rho \varphi_{n}^{-}=\psi_{n}^{+}$для функций $\varphi$ и $\psi$, получаем $(\varphi \psi)^{+}=(\varphi \psi)^{-}$; кроме того, $\varphi \psi \in \mathscr{C}(D)$ и дивизор $(\varphi \psi)_{D}=$ $\infty^{-\mathrm{g}} z_{1} \cdots z_{\mathrm{g}}$. Отсюда уже непосредственно вытекает, что $(\varphi \psi)(z) \equiv \mathrm{const} \cdot X_{\mathrm{g}}(z)$, $X_{\mathrm{g}}(z)=\prod_{j=1}^{\mathrm{g}}\left(z-z_{j}\right)$, а для функций $\varphi$ и $\psi$ получаются раздельные краевые условия: $\varphi^{+} \varphi^{-}=$const $\cdot X_{\mathrm{g}} / \rho, \psi^{+} \psi^{-}=$const $\cdot \rho X_{\mathrm{g}}$, где $X_{\mathrm{g}}$ - неизвестный полином ("полиномиальньй параметр" задачи). ${ }^{4}$ В дальнейших рассуждениях этого параграфа нам удобно временно перейти от стандартной нормировки решения $\varphi, \psi$ задачи $(\mathscr{R}) \kappa$ дpyгой, положив выше const $=1$, т.е., сохранив прежние обозначения, имеем $\varphi^{+} \varphi^{-}=$ $X_{\mathrm{g}} / \rho, \psi^{+} \psi^{-}=\rho X_{\mathrm{g}}$ (более точно, этими условиями функции $\varphi$ и $\psi$ определяются с точностью до знака \pm ; нетрудно также увидеть, что для решения модифицированной задачи имеем: $(\varphi \psi)(z) \equiv \mathrm{const} \cdot X_{\mathrm{g}}(z) r_{n}(z)$, где $\left.r_{n}(z)=\prod_{j=1}^{m}\left(z-a_{j}\right) /\left(z-a_{j, n}\right)\right)$.

Рассмотрим теперь $a$-нормированные абелевы дифференциалы третьего рода $d \Omega\left(z_{j}^{(1)}, \infty^{(2)} ; \mathbf{z}\right), j=1, \ldots, \mathrm{g}$, и $d \Omega\left(\infty^{(1)}, \infty^{(2)} ; \mathbf{z}\right)$. В многосвязной области $D^{(1)}$ они имеют периоды, кратные $2 \pi i$. Следовательно, абелевы интегралы

$$
\begin{gathered}
\Omega\left(z_{j}^{(1)}, \infty^{(2)} ; \mathbf{z}\right)=\int_{e_{2 \mathbf{g}+1}}^{\mathbf{z}} d \Omega\left(z_{j}^{(1)}, \infty^{(2)} ; \boldsymbol{\zeta}\right), \quad j=1, \ldots, \mathrm{g} \\
\Omega\left(\infty^{(1)}, \infty^{(2)} ; \mathbf{z}\right)=\int_{e_{2 \mathbf{g}+1}}^{\mathbf{z}} d \Omega\left(\infty^{(1)}, \infty^{(2)} ; \boldsymbol{\zeta}\right)
\end{gathered}
$$

в области $D^{(1)}$ однозначны по модулю $2 \pi i$ (интегрирование ведется по кривой, соединяюшей точки $e_{2 g+1}$ и $z^{(1)}$ и целиком лежащей в $\left.D^{(1)}\right)$.

Тем самым, функция

$$
F(z)=\exp \left\{(n-\mathrm{g}) \Omega\left(\infty^{(1)}, \infty^{(2)} ; z^{(1)}\right)\right\} \exp \left\{\sum_{j=1}^{\mathrm{g}} \Omega\left(z_{j}^{(1)}, \infty^{(2)} ; z^{(1)}\right)\right\}
$$

однозначна в многосвязной области $D$, а ее дивизор в $D$ имеет вид: $(F)_{D}=\infty^{n-\mathrm{g}}$. $z_{1} \cdots z_{\mathrm{g}} ;$ кроме того, $F\left(e_{2 \mathrm{~g}+1}\right)=1$.

\footnotetext{
${ }^{4}$ В классическом случае $\mathrm{g}=0$ непосредственно из последних краевых условий уже вытекает нужное представление функций $\varphi, \psi$, см. [39; с. 301].
} 
Непосредственно из явного представления абелевых дифференциалов третьего рода вытекает, что для этих дифференциалов вьполняются следующие краевые условия при $\zeta \in S$ :

$$
\begin{gathered}
d \Omega^{+}\left(z_{1}^{(1)}, \infty^{(2)} ; \zeta\right)+d \Omega^{-}\left(z_{1}^{(1)}, \infty^{(2)} ; \zeta\right)=\frac{d \zeta}{\zeta-z_{1}} \\
d \Omega^{+}\left(\infty^{(1)}, \infty^{(2)} ; \zeta\right)+d \Omega^{-}\left(\infty^{(1)}, \infty^{(2)} ; \zeta\right)=0
\end{gathered}
$$

в свою очередь, функция $F$ удовлетворяет на $S$ краевьм условиям:

$$
F^{+}(\zeta) F^{-}(\zeta)=\prod_{j=1}^{\mathrm{g}}\left(\zeta-z_{j}\right) \cdot C_{k}, \quad \zeta \in S_{k}, \quad k=1, \ldots, \mathrm{g}+1
$$

где постоянные $C_{k}=e^{c_{k}}, k=1, \ldots, \mathrm{g}$,

$$
c_{k}=c_{\mathrm{g}+1}-\int_{\mathbf{b}_{k}}\left\{\sum_{j=1}^{\mathrm{g}} d \Omega\left(z_{j}^{(1)}, \infty^{(2)} ; \mathbf{z}\right)+(n-\mathrm{g}) d \Omega\left(\infty^{(1)}, \infty^{(2)} ; \mathbf{z}\right)\right\}, \quad k=1, \ldots, \mathrm{g},
$$

a $e^{c_{\mathrm{g}+1}}=C_{\mathrm{g}+1}=1 / X_{\mathrm{g}}\left(e_{2 \mathrm{~g}+1}\right)$.

Итак, функция $F(z)$ удовлетворяет на $S$ краевым условиям $F^{+} F^{-}=C_{k} \cdot X_{\mathrm{g}}$, $\zeta \in S_{k}, k=1, \ldots, \mathrm{g}+1$, а ее дивизор в $D$ имеет вид: $(F)_{D}=\infty^{n-\mathrm{g}} \cdot z_{1} \cdots z_{\mathrm{g}}$. Следовательно, функщия $\theta(z)=\psi(z) / F(z)-$ (однозначная) голоморфная функция в $D$, которая не имеет в этой области нулей (дивизор $(\theta)_{D}=1$ ), а на границе $\partial D=S^{+} \cup S^{-}$ удовлетворяет краевому условию: $\theta^{+} \theta^{-}=\rho / C_{k}, \zeta \in S_{k}, k=1, \ldots, \mathrm{g}+1, C_{\mathrm{g}+1}=$ $1 / X_{\mathrm{g}}\left(e_{2 \mathrm{~g}+1}\right)$, остальные $C_{k}$ определяются соотношениями $(2.18)$.

Аналитическая функция $\chi(z)=\log \theta(z)$ - вообще говоря, многозначная функция в $D$; ее многозначность обусловлена только многосвязностью $D$, а периоды - целые кратные $2 \pi i$. Ясно, что при надлежашем выборе целых чисел $m_{j}, j=1, \ldots$, g, функция $2 \pi i \sum_{j=1}^{\mathrm{g}} m_{j} \Omega_{j}(z)$, где, как и ранее, $\Omega_{j}(z)=\int_{e_{2 \mathrm{~g}+1}}^{z} d \Omega_{j}(z)$, имеет те же периоды, что и функция $\chi(z)$. Тем самым, для функции

$$
\chi_{0}(z)=\chi(z)-2 \pi i \sum_{j=1}^{\mathrm{g}} m_{j} \Omega_{j}(z)
$$

имеем:

(i) $\chi_{0}(z)$ - голоморфная функция в $D$;

(ii) на $S$ вьполняется краевое условие:

$$
\begin{aligned}
& \chi_{0}^{+}+\chi_{0}^{-}=\log \rho(\zeta)-c_{\mathrm{g}+1}+2 \pi i n_{\mathrm{g}+1}, \quad \zeta \in S_{\mathrm{g}+1}, \\
& \chi_{0}^{+}+\chi_{0}^{-}=\log \rho(\zeta)-c_{k}+2 \pi i n_{k}+\sum_{j=1}^{\mathrm{g}} 2 \pi i m_{j} B_{k j}, \quad \zeta \in S_{k}, k=1, \ldots, \mathrm{g} .
\end{aligned}
$$


Отсюда немедленно вытекает, что функция $\Sigma(z)=\chi_{0}(z) / w(z)$, голоморфная в $D$ и имеющая в точке $z=\infty$ нуль кратности $g+1$, восстанавливается в $D$ интегралом типа Коши по правой части краевого условия

$$
\begin{gathered}
\Sigma^{+}-\Sigma^{-}=\frac{\log \rho(\zeta)}{w^{+}(\zeta)}-\frac{c_{\mathrm{g}+1}}{w^{+}(\zeta)}+\frac{2 \pi i n_{\mathrm{g}+1}}{w^{+}(\zeta)}, \quad \zeta \in S_{\mathrm{g}+1}, \\
\Sigma^{+}-\Sigma^{-}=\frac{\log \rho(\zeta)}{w^{+}(\zeta)}-\frac{c_{k}}{w^{+}(\zeta)}+\frac{2 \pi i n_{k}}{w^{+}(\zeta)}+\sum_{j=1}^{\mathrm{g}} \frac{2 \pi i m_{j} B_{k j}}{w^{+}(\zeta)}, \quad \zeta \in S_{k}, \\
k=1, \ldots, \mathrm{g},
\end{gathered}
$$

а нужная кратность нуля этого интеграла в бесконечно удаленной точке достигается при вьполнении g условий ортогональности

$$
\begin{gathered}
\frac{1}{\pi i} \int_{S} \log \rho(\zeta) \frac{\zeta^{\ell-1}}{w^{+}(\zeta)} d \zeta+\sum_{k=1}^{\mathrm{g}+1}\left(-c_{k} \frac{1}{\pi i} \int_{S_{k}} \frac{\zeta^{\ell-1}}{w^{+}(\zeta)} d \zeta+2 n_{k} \int_{S_{k}} \frac{\zeta^{\ell-1}}{w^{+}(\zeta)} d \zeta\right. \\
\left.+\sum_{k=1}^{\mathrm{g}} \sum_{j=1}^{\mathrm{g}} 2 m_{j} B_{k j} \int_{S_{k}} \frac{\zeta^{\ell-1}}{w^{+}(\zeta)} d \zeta\right)=0 \\
\ell=1, \ldots, \mathrm{g} .
\end{gathered}
$$

Заменяя в правой части последней формулы интегрирование по дугам $S_{k}$ на интегрирование по циклам $\mathbf{a}_{k}$, а дифференциалы $\zeta^{\ell-1} d \zeta / w(\boldsymbol{\zeta})$ на нормированные дифференциалы $d \Omega_{\ell}(\boldsymbol{\zeta})$, получаем

$$
\begin{aligned}
\frac{1}{\pi i} \int_{S} \log \rho(\zeta) d \Omega_{\ell}^{+}(\zeta) & =\frac{1}{2 \pi i}\left(c_{\ell}-c_{\mathrm{g}+1}\right)+\left(n_{\mathrm{g}+1}-n_{\ell}\right)-\sum_{j=1}^{\mathrm{g}} m_{j} B_{\ell j} \\
& =\frac{1}{2 \pi i}\left(c_{\ell}-c_{\mathrm{g}+1}\right)(\bmod \text { периодов }), \quad \ell=1, \ldots, \mathrm{g} .
\end{aligned}
$$

Воспользуемся теперь в очередной раз соотношением Римана (2.10) для того, чтобы преобразовать выражение (2.18) к виду:

$$
c_{\ell}=c_{\mathrm{g}+1}+2 \pi i\left\{\sum_{j=1}^{\mathrm{g}} \int_{z_{j}^{(1)}}^{\infty^{(2)}} d \Omega_{\ell}(\boldsymbol{\zeta})+(n-\mathrm{g}) \int_{\infty^{(1)}}^{\infty^{(2)}} d \Omega_{\ell}(\boldsymbol{\zeta})\right\} .
$$

Наконец, из (2.21) и (2.22) получаем

$$
\begin{gathered}
\sum_{j=1}^{\mathrm{g}} \int_{\infty^{(1)}}^{z_{j}^{(1)} d \Omega_{\ell}(\boldsymbol{\zeta})=}-\frac{1}{\pi i} \int_{S} \log \rho(\zeta) d \Omega_{\ell}^{+}(\zeta)+n \int_{\infty^{(1)}}^{\infty^{(2)}} d \Omega_{\ell}(\boldsymbol{\zeta}) \\
+\left(n_{\ell}-n_{\mathrm{g}+1)}-\sum_{j=1}^{\mathrm{g}} m_{j} B_{\ell j}\right. \\
\equiv-\frac{1}{\pi i} \int_{S} \log \rho(\zeta) d \Omega_{\ell}^{+}(\zeta)+n \int_{\infty}^{\infty^{(1)}} d \Omega_{\ell}(\boldsymbol{\zeta}) \\
\ell=1, \ldots, \mathrm{g}
\end{gathered}
$$

(ср. $[39 ;$ п. 4.3.1, формула $(4.3 .25)])$. 
Займемся теперь преобразованием к нужному виду последнего интеграла в выражении (2.24).

Хорошо известна $[58 ; \S 4,(4.3)]$ следующая формула для $a$-периодов дифференщиала комплексной функции Грина: $\oint_{\mathbf{a}_{k}} d G(\mathbf{z})=-2 \pi i \omega_{k}(\infty)$, где $\omega_{k}(\infty), k=1, \ldots, \mathrm{g},-$ гармоническая мера $k$-й компоненты $S_{k}$ множества $S$ относительно области $D$ в бесконечно удаленной точке, $\omega_{k}(\infty)>0, \sum_{k=1}^{\mathrm{g}+1} \omega_{k}(\infty)=1$. Непосредственно из этой формулы для $a$-периодов дифференциала $d G(\mathbf{z})$ вытекает, что справедливо следующее тождество:

$$
d G(\mathbf{z})+2 \pi i \sum_{k=1}^{\mathrm{g}} \omega_{k}(\infty) d \Omega_{k}(\mathbf{z})+d \Omega\left(\infty^{(1)}, \infty^{(2)} ; \mathbf{z}\right)=0 .
$$

Действительно, слева в последней формуле стоит голоморфньй абелев дифференщиал, все $a$-периоды которого равны нулю; следовательно, этот дифференщиал равен нулю тождественно. Отсюда и из соотношения Римана (см. (2.10)) для $b$-периодов $a$-нормированного дифференциала третьего рода

$$
\oint_{\mathbf{b}_{j}} d \Omega\left(\infty^{(1)}, \infty^{(2)} ; \mathbf{z}\right)=-2 \pi i \int_{\infty^{(1)}}^{\infty^{(2)}} d \Omega_{j}(\mathbf{z})
$$

(напомним, что интегрирование ведется по путям, лежащим в рассеченной римановой поверхности $\left.\widetilde{\Re}=\mathfrak{R} \backslash\left(\bigcup_{k=1}^{\mathrm{g}} \mathbf{a}_{k} \cup \bigcup_{k=1}^{\mathrm{g}} \mathbf{b}_{k}\right)\right)$ получаем

$$
\int_{\infty}^{\infty^{(1)}} d \Omega_{j}(\mathbf{z})=\frac{1}{2 \pi i} \oint_{\mathbf{b}_{j}} d G(\mathbf{z})+\sum_{k=1}^{\mathrm{g}} \omega_{k}(\infty) B_{k j}, \quad j=1, \ldots, \mathrm{g},
$$

где имеем $\oint_{\mathbf{b}_{j}} d G(\mathbf{z})=2 \pi i \Delta_{j}(\infty) \in i \mathbb{R}$ (см. п. 2.3). Тем самьм, $x-, y$-координаты вектора $\int_{\infty}^{\infty^{(2)}} d \Omega_{j}(\mathbf{z}), j=1, \ldots, \mathrm{g}$, в многообразии Якоби Јас $\Re$ равны соответственно $\left\{\Delta_{j}(\infty)\right\}$ и $\omega_{j}(\infty), j=1, \ldots, \mathrm{g}$, а вектора $n \int_{\infty}^{\infty^{(1)}} d \Omega_{j}(\mathbf{z})-$ соответственно $\left\{n \Delta_{j}(\infty)\right\}$ и $\left\{n \omega_{j}(\infty)\right\}$; здесь $\{\cdot\}$ обозначает дробную часть числа. Кроме того, из (2.25) для $\mathbf{z} \in \widetilde{\mathfrak{R}}$ имеем

$$
G(\mathbf{z})+2 \pi i \sum_{k=1}^{\mathrm{g}} \omega_{k}(\infty) \Omega_{k}(\mathbf{z})+\Omega\left(\infty^{(1)}, \infty^{(2)} ; \mathbf{z}\right)=0
$$

где интегрирование проводится от $e_{2 \mathrm{~g}+1}$ до $\mathbf{z}$ по произвольному пути, лежашему в $\widetilde{\Re}$.

Таким образом, из (2.24) окончательно получаем

$$
\begin{gathered}
\sum_{j=1}^{\mathrm{g}} \int_{\infty^{(1)}}^{z_{j}^{(1)}} d \Omega_{\ell}(\boldsymbol{\zeta}) \equiv \frac{i}{\pi} \int_{S} \log \rho(\zeta) d \Omega_{\ell}^{+}(\zeta)+\left\{n \Delta_{\ell}(\infty)\right\}+\sum_{k=1}^{\mathrm{g}}\left\{n \omega_{k}(\infty)\right\} B_{\ell k} \\
\ell=1, \ldots, \mathrm{g} .
\end{gathered}
$$

Необходимость разрешимости проблемы обрашения Якоби (2.15) доказана. 
Нетрудно видеть, что проведенные рассуждения обратимы, так что достаточность условия (2.15) также имеет место.

Для доказательства (с) отметим сначала, что равенства (2.23) - точные. Совместньй анализ $(2.23)$ и (2.28) показьвает, что если все точки $z_{j}^{(1)}$ компактно по $n \rightarrow \infty$ принадлежат области $D$, то $m_{k}=\left[n \omega_{k}(\infty)\right]+N_{k}$, где целые числа $N_{k}=N_{k}(n)$ ограничены в совокупности при $n \rightarrow \infty$ (здесь [ · ] - целая часть числа).

Запишем теперь формулы (2.20) для скачка функщии $\Sigma$ в виде

$$
\begin{aligned}
\Sigma^{+}-\Sigma^{-}= & \frac{\log \rho(\zeta)}{w^{+}(\zeta)}-\frac{c_{\mathrm{g}+1}}{w^{+}(\zeta)}+\frac{2 \pi i n_{\mathrm{g}+1}}{w^{+}(\zeta)}, \quad \zeta \in S_{\mathrm{g}+1} \\
\Sigma^{+}-\Sigma^{-}= & \frac{\log \rho(\zeta)}{w^{+}(\zeta)}-\frac{c_{\mathrm{g}+1}}{w^{+}(\zeta)}+\frac{2 \pi i n_{\mathrm{g}+1}}{w^{+}(\zeta)}-\frac{c_{k}-c_{\mathrm{g}+1}}{w^{+}(\zeta)} \\
& +\frac{2 \pi i\left(n_{k}-n_{\mathrm{g}+1}\right)}{w^{+}(\zeta)}+\sum_{j=1}^{\mathrm{g}} \frac{2 \pi i m_{j} B_{k j}}{w^{+}(\zeta)}, \quad \zeta \in S_{k}, \quad k=1, \ldots, \mathrm{g} .
\end{aligned}
$$

Первая часть (2.21) - точное равенство, поэтому последние краевые условия приводятся к виду

$$
\begin{gathered}
\Sigma^{+}-\Sigma^{-}=\frac{\log \rho(\zeta)}{w^{+}(\zeta)}-\frac{c_{\mathrm{g}+1}}{w^{+}(\zeta)}+\frac{2 \pi i n_{\mathrm{g}+1}}{w^{+}(\zeta)}, \quad \zeta \in S_{\mathrm{g}+1} \\
\Sigma^{+}-\Sigma^{-}=\frac{\log \rho(\zeta)}{w^{+}(\zeta)}-\frac{c_{\mathrm{g}+1}}{w^{+}(\zeta)}+\frac{2 \pi i n_{\mathrm{g}+1}}{w^{+}(\zeta)}+\frac{\lambda_{k}}{w^{+}(\zeta)}, \quad \zeta \in S_{k} \\
k=1, \ldots, \mathrm{g} \\
\text { где } \lambda_{k}=-2 \int_{S} \log \rho(t) d \Omega_{k}^{+}(t)
\end{gathered}
$$

(величины $\lambda_{k}$ определены однозначно по модулю $2 \pi i$ ).

Из (2.17), (2.19) и (2.29) получаем, что для $\psi(z)=F(z) \theta(z)=F(z) e^{\chi(z)}$ справедливо представление

$$
\begin{aligned}
\psi(z)= & \exp \left\{-\frac{1}{2} c_{\mathrm{g}}+(n-\mathrm{g}) \Omega\left(\infty^{(1)}, \infty^{(2)} ; z^{(1)}\right)+\sum_{j=1}^{\mathrm{g}} \Omega\left(z_{j}^{(1)}, \infty^{(2)} ; z^{(1)}\right)\right\} \\
& \times \exp \left\{2 \pi i \sum_{k=1}^{\mathrm{g}} m_{k} \Omega_{k}(z)\right\} \\
& \times \exp \left\{w(z) \frac{1}{2 \pi i} \int_{S} \frac{\log \rho(\zeta)}{z-\zeta} \frac{d \zeta}{w^{+}(\zeta)}\right\} \\
& \times \exp \left\{w(z) \frac{1}{2 \pi i} \sum_{k=1}^{\mathrm{g}} \lambda_{k} \int_{S_{k}} \frac{1}{z-\zeta} \frac{d \zeta}{w^{+}(\zeta)}\right\} .
\end{aligned}
$$

Отметим, что классическому случаю $\mathrm{g}=0$ и $S=[-1,1]$ соответствуют $\lambda_{1}=0$ и

$$
\theta(z)=\exp \left\{\frac{\sqrt{z^{2}-1}}{2 \pi} \int_{-1}^{1} \frac{\log \rho(x)}{z-x} \frac{d x}{\sqrt{1-x^{2}}}\right\}
$$


- классическая функция Сегё. Случай, когда $S$ есть объединение непересекающихся интервалов вещественной оси, а вес $\rho$ такой, что $\rho / i \sqrt{h}>0$ и вьполняются условия ортогональности

$$
\int_{S} \log \rho(t) d \Omega_{k}^{+}(t)=0, \quad k=1, \ldots, \mathrm{g},
$$

фактически рассматривался Ахиезером [38], [60], который для такого веса ввел оператор

$$
A(z ; \rho)=\exp \left\{\sqrt{h(z)} \frac{1}{2 \pi} \int_{S} \frac{\log \rho(x)}{z-x} \frac{d x}{\sqrt{-h(x)}}\right\}
$$

(в выражении $\sqrt{-h(x)}$ под $\sqrt{ }$ понимается арифметический корень).

Воспользуемся теперь тем, что $m_{k}=\left[n \omega_{k}(\infty)\right]+N_{k}$, где целые числа $N_{k}=N_{k}(n)$ ограничены в совокупности при $n \rightarrow \infty$. Из представления (2.30) с помощью (2.27) получаем для функции $\psi$ :

$$
\psi(z)=\exp \{-(n-\mathrm{g}) G(z, \infty)\} W(z)=\Phi(z)^{-(n-\mathrm{g})} W(z)
$$

где многозначная функшия

$$
\begin{aligned}
W(z)= & \exp \left\{-\frac{1}{2} c_{\mathrm{g}+1}+2 \pi i \sum_{k=1}^{\mathrm{g}}\left(\mathrm{g}-\left\{n \omega_{k}(\infty)\right\}\right) \Omega_{k}(z)\right\} \\
& \times \exp \left\{\sum_{j=1}^{\mathrm{g}} \Omega\left(z_{j}^{(1)}, \infty^{(2)} ; z^{(1)}\right)\right\} \exp \left\{w(z) \frac{1}{2 \pi i} \int_{S} \frac{\log \rho(\zeta)}{z-\zeta} \frac{d \zeta}{w^{+}(\zeta)}\right\} \\
& \times \exp \left\{w(z) \frac{1}{2 \pi i} \sum_{k=1}^{\mathrm{g}} \lambda_{k} \int_{S_{k}} \frac{1}{z-\zeta} \frac{d \zeta}{w^{+}(\zeta)}\right\}
\end{aligned}
$$

зависит только от "геометрии" задачи и от класса эквивалентности $[\rho]$; кроме того, она равномерно ограничена по модулю внутри области $D$. Соответствующее представление получаем и для функции $\varphi(z)=X_{\mathrm{g}}(z) / \psi(z)$.

Наконец, вспомним, что мы временно изменили нормировку решения задачи $(\mathscr{R})$. С учетом этого замечания получаем, что равномерно внутри $D$

$$
\varphi_{n}(z ; \rho)=\operatorname{cap}(S)^{n} \cdot \Phi(z)^{n} F_{n}(z ; \rho),
$$

где функция $F_{n}$ обладает теми же свойствами, что и функция $W$. Аналогично, равномерно внутри $D$

$$
\psi_{n}(z ; \rho)=\operatorname{cap}(S)^{n} \cdot \Phi(z)^{-n} \frac{X_{\mathrm{g}, n}(z)}{F_{n}(z ; \rho)}
$$

Основная лемма доказана. 


\section{§4. Построение "явного" решения проблемы обращения Якоби}

4.1. Разрешимость проблемы обращения Якоби в случае "общего положения" $\left(C_{1}\right)-\left(C_{2}\right)$ (случай (II)). По теореме Кронекера из рациональной независимости периодов функции Грина с числом $2 \pi i$ (ср. [58; с. 190, 191]) вытекает, что правая часть (2.15) всюду плотна в Јас $\mathfrak{R}$ при $n \rightarrow \infty$. С другой стороны, как хорошо известно [49]-[51], отображение Абеля $\mathfrak{A}: S^{\mathrm{g}} \mathfrak{R} \rightarrow$ Јас $\mathfrak{R}$ является сюръективным и локально биголоморфным. Отсюда уже легко вытекает существование требуемой подпоследовательности. Действительно, выберем множество $\mathscr{U}$ в $S^{\mathrm{g}} \mathfrak{R}$ так, чтобы оно было устроено следуюшим образом: $\mathscr{U}$ представляет собой (неупорядоченное) произведение g непересекаюшихся замкнутых кругов $\mathscr{U}_{j}$ в $D^{(1)}, \mathscr{U}_{j} \cap \mathscr{U}_{k}=\varnothing$ при $j \neq k$. В соответствии со свойствами отображения Абеля $\mathfrak{A}^{-1}(\mathfrak{A}(\mathscr{U}))=\mathscr{U}$ и ограничение $\mathfrak{A}$ на $\mathscr{U}$ - биекция, т.е. проблема обрашения Якоби $(2.6)$ при $\mathbf{v} \in \mathfrak{A}(\mathscr{U})$ имеет единственное решение в $S^{g} \mathfrak{R}$, причем все соответствуюшие точки $\mathbf{z}_{j}=z_{j}^{(1)} \in \mathscr{U}_{j}$ и, тем самым, попарно различны.

Так как правая часть (2.15) всюду плотна в Јас $\mathfrak{R}$ при $n \rightarrow \infty$, то по некоторой бесконечной подпоследовательности $\Lambda \subset \mathbb{N}$ эта правая часть принадлежит $\mathfrak{A}(\mathscr{U})$. Следовательно, соответствуюшие точки $\mathbf{z}_{j, n}-$ решение проблемы обрашения Якоби - принадлежат первому листу: $\mathbf{z}_{j, n}=z_{j, n}^{(1)} \in \mathscr{U}_{j}, j=1, \ldots, \mathrm{g}$. Разрешимость проблемы обрашения Якоби в нужном нам смысле доказана.

4.2. Разрешимость проблемы обращения Якоби в случае (I). Напомним, что для $b$-периодов $a$-нормированного абелева дифференщиала $d \Omega\left(\mathbf{z}_{1}, \mathbf{z}_{2} ; \mathbf{z}\right)$ справедливо соотношение Римана:

$$
\int_{\mathbf{b}_{j}} d \Omega\left(\mathbf{z}_{1}, \mathbf{z}_{2} ; \zeta\right)=-2 \pi i \int_{\mathbf{z}_{1}}^{\mathbf{z}_{2}} d \Omega_{j}(\boldsymbol{\zeta}), \quad j=1, \ldots, \mathrm{g}
$$

в частности,

$$
\int_{\mathbf{b}_{j}} d \Omega\left(z_{0}^{(1)}, z_{0}^{(2)} ; \zeta\right)=-2 \pi i \int_{z_{0}^{(1)}}^{z_{0}^{(2)}} d \Omega_{j}(\boldsymbol{\zeta}), \quad j=1, \ldots, \mathrm{g}
$$

и

$$
\int_{\mathbf{b}_{j}} d \Omega\left(\infty^{(1)}, \infty^{(2)} ; \boldsymbol{\zeta}\right)=-2 \pi i \int_{\infty}^{\infty^{(2)}} d \Omega_{j}(\boldsymbol{\zeta}), \quad j=1, \ldots, \mathrm{g} ;
$$

как обычно, интегрирование ведется по путям, лежашим в рассеченной римановой поверхности $\widetilde{\Re}$. Подчеркнем, что в рассматриваемом случае $a$-циклы - это отрезки $\left[e_{2 j-1}, e_{2 j}\right], j=1, \ldots, \mathrm{g}$, проходимые дважды: по “верхнему" и по "нижнему" берегам. Соответствующие $b$-циклы устроены следующим образом. $j$-й $b$-цикл "состоит" из лакун $\left[e_{2 k}, e_{2 k+1}\right], k=j, \ldots, \mathrm{g}$, проходимых дважды: по первому листу в направлении от $e_{2 k}$ к $e_{2 k+1}$ и по второму листу - в противоположном направлении (тем самым, $j$-й $b$-цикл состоит из g $-j$ циклов, “зацепленных" за $a$-циклы $\left.\mathbf{a}_{k}, k=j+1, \ldots, \mathrm{g}\right)$. Под $\int_{\infty}^{z^{(1)}}$ будем понимать интеграл по пути, расположенному целиком на вещественной оси и идущему “справа налево” от точки $+\infty$ до точки $z_{j}^{(1)}=z_{j} \in\left(e_{2 j}, e_{2 j+1}\right)$, 
$j=1, \ldots$, g. Тем самым,

$$
\begin{aligned}
\int_{\infty}^{z_{j}^{(1)}} d \Omega_{\ell}^{+}(\zeta) & =\int_{\infty}^{e_{2 \mathrm{~g}+2}(1)} d \Omega_{\ell}^{+}(\zeta)+\int_{e_{2 \mathrm{~g}+2}}^{e_{2 \mathrm{~g}+1}} d \Omega_{\ell}^{+}(\zeta)+\int_{e_{2 \mathrm{~g}+1}}^{z_{j}^{(1)}} d \Omega_{\ell}^{+}(\zeta) \\
& =\frac{1}{2} \int_{\infty^{(1)}}^{\infty^{(2)}} d \Omega_{\ell}(\zeta)-\int_{S_{\mathrm{g}+1}} d \Omega_{\ell}^{+}(\zeta)+\int_{e_{2 \mathrm{~g}+1}}^{z_{j}^{(1)}} d \Omega_{\ell}^{+}(\zeta) \\
& =\frac{1}{2} \int_{\infty(1)}^{\infty^{(2)}} d \Omega_{\ell}(\zeta)+\frac{1}{2}+\int_{e_{2 \mathrm{~g}+1}}^{z_{j}^{(1)}} d \Omega_{\ell}^{+}(\zeta) \\
& =\frac{1}{2}\left(\frac{1}{2 \pi i} \oint_{\mathbf{b}_{\ell}} d G(\zeta)+\sum_{k=1}^{\mathrm{g}} \omega_{k}(\infty) B_{\ell k}\right)+\frac{1}{2}+\int_{e_{2 \mathrm{~g}+1}}^{z_{j}^{(1)}} d \Omega_{\ell}^{+}(\zeta)
\end{aligned}
$$

Из $(2.31)$ и $(2.32)$ для $z_{j}^{(1)}=z_{j} \in\left(e_{2 j}, e_{2 j+1}\right), j=1, \ldots, \mathrm{g}$, получаем

$$
\begin{aligned}
\int_{e_{2 \mathrm{~g}+1}}^{z_{j}^{(1)}} d \Omega_{\ell}^{+}(\zeta) & =\left\{\int_{e_{2 \mathrm{~g}+1}}^{e_{2 j}}+\int_{e_{2 j}}^{z_{j}^{(1)}}\right\} d \Omega_{\ell}^{+}(\zeta)=-\frac{1}{2} B_{j \ell}-\frac{1}{2} \theta_{\ell j}+\int_{e_{2 j}}^{z_{j}^{(1)}} d \Omega_{\ell}^{+}(\zeta), \\
\int_{z_{j}^{(2)}}^{z_{j}^{(1)}} d \Omega_{\ell}(\zeta) & =\left\{\int_{e_{2 j}^{(2)}}^{e_{2 j}^{(1)}}-2 \int_{z_{j}^{(1)}}^{e_{2 j}^{(1)}}\right\} d \Omega_{\ell}^{+}(\zeta)=-B_{j \ell}+2 \int_{e_{2 j}^{(1)}}^{z_{j}^{(1)}} d \Omega_{\ell}^{+}(\zeta),
\end{aligned}
$$

где

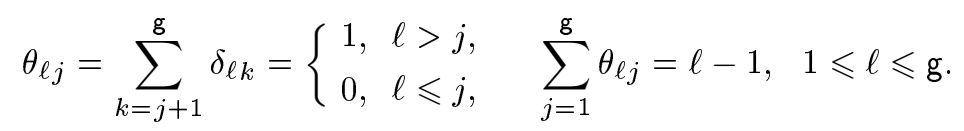

Если теперь мы подставим (2.34) в (2.33), то получим

$$
\int_{\infty(1)}^{z_{j}^{(1)}} d \Omega_{\ell}^{+}(\zeta)=\frac{1}{2} \sum_{k=1}^{\mathrm{g}} \omega_{k}(\infty) B_{\ell k}+\frac{1}{2}-\frac{1}{2} B_{j \ell}-\frac{1}{2} \theta_{\ell j}+\int_{e_{2 j}}^{z_{j}^{(1)}} d \Omega_{\ell}^{+}(\zeta) .
$$

Следовательно,

$$
\begin{aligned}
\sum_{j=1}^{\mathrm{g}} \int_{\infty(1)}^{z_{j}^{(1)}} d \Omega_{\ell}^{+}(\zeta) & =\frac{\mathrm{g}}{2} \sum_{s=1}^{\mathrm{g}} \omega_{s}(\infty) B_{\ell s}+\frac{\mathrm{g}}{2}-\frac{1}{2} \sum_{j=1}^{\mathrm{g}} B_{j \ell}-\frac{1}{2} \sum_{j=1}^{\mathrm{g}} \theta_{\ell j}+\sum_{j=1}^{\mathrm{g}} \int_{e_{2 j}}^{z_{j}^{(1)}} d \Omega_{\ell}^{+}(\zeta) \\
& =\frac{\mathrm{g}}{2} \sum_{s=1}^{\mathrm{g}} \omega_{s}(\infty) B_{\ell s}-\frac{1}{2} \sum_{j=1}^{\mathrm{g}} B_{j \ell}+\frac{\mathrm{g}-\ell+1}{2}+\sum_{j=1}^{\mathrm{g}} \int_{e_{2 j}}^{z_{j}^{(1)}} d \Omega_{\ell}^{+}(\zeta) .
\end{aligned}
$$

Как уже отмечалось, функция $\widehat{\rho}(z ; h)$ вида $(2.5)$ - марковская при условии, что $i \rho / \sqrt{h}>0$ на $S$, т.е. для выбранной вьше ветви корня $\sqrt{h(z)}$ имеем: $\arg \rho(\zeta)=$ $\arg \sqrt{h(\zeta)}+\pi / 2, \zeta \in S$; в частности, $\arg \rho(\zeta)=\pi$ при $\zeta \in S_{\mathrm{g}+1}$ (напомним, что $\sqrt{h(z)}>0$ при $\left.z>e_{2 \mathrm{~g}+2}\right)$. Следовательно,

$$
\begin{aligned}
\int_{S} \log \rho(\zeta) d \Omega_{\ell}^{+}(\zeta) & =\int_{S} \log |\rho(\zeta)| d \Omega_{\ell}^{+}(\zeta)+\sum_{k=1}^{\mathrm{g}+1}(\mathrm{~g}+1-k+1) \pi i \int_{S_{k}} d \Omega_{\ell}^{+}(\zeta) \\
& =\int_{S} \log |\rho(\zeta)| d \Omega_{\ell}^{+}(\zeta)+\frac{\mathrm{g}-\ell+1}{2} \pi i
\end{aligned}
$$


Таким образом, система (2.15) эквивалентна следующей системе относительно точек $z_{j}^{(1)}=z_{j, n}^{(1)}, \ell=1, \ldots, \mathrm{g}$ :

$$
\begin{aligned}
\sum_{j=1}^{\mathrm{g}} \int_{e_{2 j}^{(1)}}^{z_{j}^{(1)}} d \Omega_{\ell}(\boldsymbol{\zeta}) \equiv & -\frac{\mathrm{g}}{2} \sum_{k=1}^{\mathrm{g}} \omega_{k}(\infty) B_{\ell k}+\frac{1}{2} \sum_{k=1}^{\mathrm{g}} B_{\ell k} \\
& +\frac{i}{\pi} \int_{S} \log |\rho(\zeta)| d \Omega_{\ell}^{+}(\zeta)+\sum_{k=1}^{\mathrm{g}}\left\{n \omega_{k}(\infty)\right\} B_{\ell k}
\end{aligned}
$$

Нетрудно видеть, что из условия каноничности базиса дифференциалов $d \Omega_{\ell}(\boldsymbol{\zeta})$ (относительно заданных базисных циклов $\left\{\mathbf{a}_{j}, \mathbf{b}_{j}\right\}$ в вытекает, что каждый дифференшиал $d \Omega_{\ell}(\boldsymbol{\zeta})$ вещественнозначен на $S$ и имеет ровно один нуль в каждом интервале $\left(e_{2 j-1}, e_{2 j}\right), j=1, \ldots, \mathrm{g}+1, j \neq \ell$. В лакунах $\left(e_{2 j}, e_{2 j+1}\right), j=1, \ldots, \mathrm{g}$, эти дифференциалы принимают чисто мнимые значения, отличные от нуля. Отсюда вытекает, во-первых, что все $B_{\ell k}$ чисто мнимые и, во-вторых, что и левая и правая части (2.36) также чисто мнимые. Кроме того, матрица $\left\|B_{\ell k}\right\|$ невырождена. Следовательно, система сравнений

$$
\sum_{k=1}^{\mathrm{g}} y_{k} B_{\ell k}=\sum_{j=1}^{\mathrm{g}} \int_{e_{2 j}^{(1)}}^{z_{j}^{(1)}} d \Omega_{\ell}(\boldsymbol{\zeta}) \quad(\bmod \text { периодов }), \quad \ell=1, \ldots, \mathrm{g},
$$

относительно вещественных величин $y_{1}, \ldots, y_{\mathrm{g}}$ имеет (единственное) решение такое, что все $y_{j} \in[0,1)$. Аналогично, существуют вешественные величины $\rho_{1}, \ldots, \rho_{\mathrm{g}}$ такие, что

$$
\sum_{k=1}^{\mathrm{g}} \rho_{k} B_{\ell k}=\frac{i}{\pi} \int_{S} \log |\rho(\zeta)| d \Omega_{\ell}^{+}(\zeta) \in i \mathbb{R}, \quad \ell=1, \ldots, \mathrm{g} .
$$

Тем самьм, (2.36) эквивалентно системе сравнений

$$
\sum_{k=1}^{\mathrm{g}} y_{k} B_{\ell k} \equiv-\frac{\mathrm{g}}{2} \sum_{k=1}^{\mathrm{g}} \omega_{k}(\infty) B_{\ell k}+\frac{1}{2} \sum_{k=1}^{\mathrm{g}} B_{\ell k}+\sum_{k=1}^{\mathrm{g}} \rho_{k} B_{\ell k}+\sum_{k=1}^{\mathrm{g}}\left\{n \omega_{k}(\infty)\right\} B_{\ell k},
$$

или

$$
y_{k}=-\frac{\mathrm{g}}{2} \omega_{k}(\infty)+\frac{1}{2}+\rho_{k}+n \omega_{k}(\infty) \quad(\bmod 1),
$$

где, подчеркнем, величины $y_{1}, \ldots, y_{\mathrm{g}}, y_{j} \in[0,1)$, определяются точками $z_{j}^{(1)} \in$ $\left(e_{2 j}, e_{2 j+1}\right), j=1, \ldots, \mathrm{g}$. Так как по условию числа $\omega_{1}(\infty), \ldots, \omega_{\mathrm{g}+1}(\infty)$ рационально независимы, то рассуждения, подобные использованньм вьше в п. 4.1, доказывают нужный нам результат.

Отметим, что для величин $\rho_{k}$ справедливо представление

$$
\rho_{k}=\frac{1}{\pi} \int_{S} \log |\rho(\zeta)| \frac{\partial \omega_{k}(\zeta)}{\partial n_{\zeta}^{+}} d \zeta, \quad k=1, \ldots, \mathrm{g}
$$


(здесь и ниже $\partial / \partial n_{\zeta}$ означает производную по внутренней нормали к границе области $D)$, а система (2.36) приводится к виду

$$
\sum_{j=1}^{\mathrm{g}} \omega_{k}\left(z_{j}\right)=(\mathrm{g}-2 n) \omega_{k}(\infty)-\frac{2}{\pi} \int_{S} \log |\rho(\zeta)| \frac{\partial \omega_{k}(\zeta)}{\partial n_{\zeta}^{+}} d \zeta \quad(\bmod 2), \quad k=1, \ldots, \mathrm{g}
$$

(cр. [58] и [32; лемма 3]). Тем самым, здесь фактически доказано, что по некоторой подпоследовательности система сравнений (2.37) имеет решение $z_{1}, \ldots, z_{\mathrm{g}}$ такое, ито все $z_{j} \in\left(e_{2 j}, e_{2 j+1}\right)$.

4.3. Доказательство следствия. Легко видеть, что вопрос о разрешимости модифицированной краевой задачи Римана эквивалентен проблеме обрашения Якоби вида (2.15) относительно точек $z_{1, n}, \ldots, z_{\mathrm{g}, n}$, где к правой части добавлены слагаемые

$$
\sum_{j=1}^{m} \int_{a_{j}^{(1)}}^{a_{j, n}^{(2)}} d \Omega_{k} \rightarrow \sum_{j=1}^{m} \int_{a_{j}^{(1)}}^{a_{j}^{(2)}} d \Omega_{k} \quad \text { при } n \rightarrow \infty, \quad k=1, \ldots, \mathrm{g} .
$$

В случае (II) утверждение следствия доказьвается по схеме, проведенной в п. 4.1.

В случае (I) доказательство проводится по схеме п. 4.2 со следуюшими изменениями. Точки $z_{1}, z_{2}, \ldots, z_{\mathrm{g}}$ надо “искать" теперь в окрестности лакун $\left(e_{2 j}, e_{2 j+1}\right)$, используя упомянутые в п. 4.2 свойства дифференциалов $d \Omega_{k}(\boldsymbol{\zeta}):$ каждый дифференциал $d \Omega_{k}(\zeta)$ вешественнозначен на $S=\bigcup_{j=1}^{\mathrm{g}+1}\left[e_{2 j-1}, e_{2 j}\right]$ и принимает чисто мнимые значения, отличные от нуля, в лакунах $\left(e_{2 j}, e_{2 j+1}\right), j=1, \ldots, \mathrm{g}$. Тем самым, $d \Omega_{k}(\mathbf{z})=$ $p_{k}(z) d z / w(\mathbf{z})$, где $p_{k} \in i \mathbb{R}[z]$. Отсюда уже легко вытекает, что

$$
\operatorname{Re} \int_{e_{2 \mathrm{~g}+1}}^{\bar{z}^{(1)}} d \Omega_{k}=\operatorname{Re} \int_{e_{2 \mathrm{~g}+1}}^{z^{(2)}} d \Omega_{k}, \quad k=1, \ldots, \mathrm{g} .
$$

Завершаются рассуждения так же, как и в п. 4.2, ссылкой на теорему Кронекера.

\section{§5. Доказательство основных результатов}

5.1. Случай, когда допустимый вес $\rho$ аналитичен на $S$ : асимптотическая формула для полиномов Паде вне множества $S$. Пусть $f=\widehat{\rho}+r, f \in \mathscr{N}$ или $f \in \mathscr{N}^{\circ}$ и вьполняются соответствуюшие условия "общего положения". Пусть $\left\{a_{1}, \ldots, a_{m}\right\}$ - полюсы функщии $f$ в $D$. Из сходимости главной диагонали $[n / n]_{f}$ по емкости к функции $f$ внутри области $D$ вытекает, что по крайней мере $m$ полюсов рациональной функции $[n / n]_{f}$ (нулей полинома $Q_{n}$ ) сходятся к $m$ полюсам функции $f$ в $D$. Обозначим эти нули полинома $Q_{n}$ через $a_{1, n}, \ldots, a_{m, n}$. Так как выполнены условия "общего положения", то в соответствии со следствием $\S 3$ для всех $n$ из некоторой подпоследовательности $\Lambda \subset \mathbb{N}$ существует решение $\varphi_{n}, \psi_{n}$ модифицированной краевой задачи Римана, где в качестве параметров $a_{1}, \ldots, a_{m}$ и $a_{1, n}, \ldots, a_{m, n}$ выбраны соответственно полюсы функций $f$ и $[n / n]_{f}$.

Непосредственно из формулы Сохоцкого для предельных значений интеграла типа Коши на контуре интегрирования вытекает следуюшее соотношение для функции остатка $R_{n}(z)=\left(Q_{n} f-P_{n}\right)(z)$ на множестве $S$ :

$$
R_{n}^{+}(\zeta)-R_{n}^{-}(\zeta)=-\frac{Q_{n}(\zeta) \rho(\zeta)}{w^{+}(\zeta)}, \quad \zeta \in S \backslash\left\{e_{1}, \ldots, e_{2 \mathrm{~g}+2}\right\}
$$


Из этого соотношения, используя краевые условия, которьм удовлетворяют на $S$ функции $\varphi_{n}$ и $\psi_{n}$, легко получить следующую пару соотношений:

$$
\begin{array}{ll}
\left(R_{n} \varphi_{n} w\right)^{+}(\zeta)+\left(R_{n} \psi_{n} w\right)^{-}(\zeta) \frac{1}{\rho(\zeta)}=-Q_{n}(\zeta) \psi_{n}^{-}(\zeta), & \zeta \in S \\
\left(R_{n} \varphi_{n} w\right)^{-}(\zeta)+\left(R_{n} \psi_{n} w\right)^{+}(\zeta) \frac{1}{\rho(\zeta)}=-Q_{n}(\zeta) \psi_{n}^{+}(\zeta), \quad \zeta \in S
\end{array}
$$

Определим теперь на двулистной римановой поверхности $\mathfrak{R}$ кусочно-мероморфную функцию $F(\mathbf{z})$ следуюшим образом:

$$
F(\mathbf{z})= \begin{cases}R_{n}(z) \varphi_{n}(z) w(z), & \mathbf{z} \in D^{(1)}, \\ -Q_{n}(z) \psi_{n}(z), & \mathbf{z} \in D^{(2)} .\end{cases}
$$

Нетрудно увидеть, что в бесконечно удаленных точках $\mathbf{z}=\infty^{(1)}$ и $\mathbf{z}=\infty^{(2)}$ функция $F$ имеет полюсы порядка $\leqslant$ g; в остальных точках $\mathfrak{R} \backslash \Gamma$ функция $F$ голоморфна. Непосредственно из (2.38) вытекает, что на кривой $\Gamma$ для $F$ вьполняется граничное условие

$$
F(\boldsymbol{\zeta}+)-F(\boldsymbol{\zeta}-)=\chi(\boldsymbol{\zeta}-) \frac{1}{\rho(\zeta)}, \quad \zeta \in \Gamma,
$$

где мы положили $\chi(\mathbf{z})=-R_{n}(z) \psi_{n}(z) w(\mathbf{z}), \mathbf{z} \in D^{(2)}$. Как известно (см. п. 2.5), с помощью мероморфного абелева дифференциала

$$
d \Omega(\mathbf{z} ; \boldsymbol{\zeta})=\frac{1}{2} \frac{w(\boldsymbol{\zeta})+w(\mathbf{z})}{\zeta-z} \frac{d \zeta}{w(\boldsymbol{\zeta})},
$$

заданного на римановой поверхности $\mathfrak{R}$, функщия $F(\mathbf{z})$ восстанавливается по граничным условиям (2.39) интегралом типа Коши:

$$
F(\mathbf{z})=-\frac{1}{2 \pi i} \int_{\Gamma^{+}} \chi(\boldsymbol{\zeta}-) \frac{1}{\rho(\zeta)} d \Omega(\mathbf{z} ; \boldsymbol{\zeta})-p_{n}(z), \quad \mathbf{z} \notin \Gamma,
$$

где $p_{n}(z) \in \mathbb{C}[z], \operatorname{deg} p_{n} \leqslant$ g. Это и есть то самое сингулярное интегральное уравнение для полиномов Паде (точнее, для функщии остатка $R_{n}$ ), о котором говорилось в начале этой главы.

Рассмотрим это уравнение для $\mathbf{z} \in D^{(1)}$. Так как $\rho(\zeta)$ голоморфна на каждой связной компоненте $S_{j}$ компакта $S$, то $\rho(\zeta)$ голоморфина на каждой кривой $\Gamma_{j}$. Поэтому контур Г можно, не меняя значения интеграла, покомпонентно продеформировать в близкий контур $\Gamma^{\prime}$, также состоящий из $\mathrm{g}+1$ компоненты и целиком расположенньй на втором листе $D^{(2)}$ в некоторой окрестности $Г$, в которой определена и голоморфна функция $\rho(\zeta)$ (напомним, что функция $\chi(\zeta)$ определена и голоморфна на всем втором листе $\left.D^{(2)}\right)$. Полученная интегральная формула задает аналитическое продолжение функции $F(\mathbf{z}), \mathbf{z} \in D^{(1)}$, через контур $Г$ на второй лист римановой поверхности вплоть до контура $\Gamma^{\prime}$ (отметим, что для $\mathbf{z} \in D^{(2)}$ оно не совпадает с функцией $F(\mathbf{z}), \mathbf{z} \in D^{(2)}$, определенной выше). Отсюда, учитьвая определения функций $F$ и $\chi$, получаем:

$$
\begin{aligned}
\left(R_{n} \varphi_{n} w\right)(z) & =\frac{1}{2 \pi i} \int_{\Gamma^{\prime}}\left(R_{n} \psi_{n} w\right)(\zeta) \frac{1}{\rho(\zeta)} d \Omega\left(z^{(1)} ; \zeta^{(2)}\right)-p_{n}(z) \\
& =\frac{1}{2 \pi i} \int_{\Gamma^{\prime}}\left(R_{n} \varphi_{n} w\right)(\zeta) \frac{\psi_{n}(\zeta)}{\varphi_{n}(\zeta)} \frac{1}{\rho(\zeta)} d \Omega\left(z^{(1)} ; \zeta^{(2)}\right)-p_{n}(z), z \in \bar{D}
\end{aligned}
$$


Выберем теперь и зафиксируем произвольное достаточно малое $\varepsilon>0$, удовлетворяющее следуюшим условиям: линия уровня функции Грина $L(\varepsilon)=\left\{z: g_{D}(z, \infty)=\varepsilon\right\}$ состоит ровно из $\mathrm{g}+1$ компоненты; функция $\rho$ голоморфна внутри и на $L(\varepsilon)$; все точки $a_{j}$ лежат в области $D(\varepsilon)=\left\{z: g_{D}(z, \infty)>\varepsilon\right\}$, а точки $a_{j, n}, z_{j, n} \in D(\varepsilon)$ для достаточно больших $n \in \Lambda$. Только такие $n \in \Lambda$ мы и рассматриваем в дальнейшем. Последовательность $\left\{\varepsilon_{n}\right\}_{n \in \Lambda}, \varepsilon_{n} \in[\varepsilon / 2,3 \varepsilon / 4]$, выберем, исходя из следуюшего условия:

$$
\max _{z \in L\left(\varepsilon_{n}\right)}\left|p_{n}(z)\right|=O(1) \cdot \min _{z \in L\left(\varepsilon_{n}\right)}\left|p_{n}(z)\right|
$$

при $n \rightarrow \infty, n \in \Lambda$ (это, очевидно, возможно, так как $\operatorname{deg} p_{n} \leqslant \mathrm{~g}$ ).

Пусть теперь контур $\Gamma^{\prime}=\Gamma^{\prime}(n) \subset D^{(2)}$ в $(2.41)$ таков, что в области $D$ ему соответствует линия уровня $L\left(\varepsilon_{n}\right)$. Подчеркнем еще раз, что формула $(2.41)$ справедлива в замкнутой области $\overline{D^{(1)}}$, т.е. остается верной и для $z=z^{(1)} \in \Gamma$ : непосредственно из явного вида $(2.14)$ дифференциала $d \Omega\left(z^{(1)} ; \zeta^{(2)}\right)$ вытекает, что он не имеет особенности при $z=\zeta$.

С учетом того, что голоморфная в $\mathbb{C} \backslash S$ функция $\left(R_{n} \varphi_{n} w\right)(z)$ имеет полюс в точке $z=\infty$ порядка $\leqslant \mathrm{g}$, и явного представления функций $\varphi_{n}$ и $\psi_{n}$ получаем из $(2.41)$ следуюшее соотношение для максимума модуля $M_{n}$ функции $\left(R_{n} \varphi_{n} w\right)(z)$ на $S^{+} \cup S^{-}$:

$$
M_{n}=M_{n} \cdot O\left(q^{n}\right)+\max _{z \in S}\left|p_{n}(z)\right|, \quad n \in \Lambda, \quad n \rightarrow \infty
$$

$q=\exp \left\{-2 \varepsilon_{n}\right\} \in(0,1)$. Следовательно, $M_{n}=\max _{z \in S}\left|p_{n}(z)\right|\left(1+O\left(q^{n}\right)\right), n \in \Lambda$, $n \rightarrow \infty$, и, кроме того, $\max _{z \in S}\left|\left(R_{n} \varphi_{n} w\right)(z)-p_{n}(z)\right|=M_{n} \cdot O\left(q^{n}\right)$.

Рассмотрим теперь интегральное уравнение $(2.40)$ для $\mathbf{z} \in \overline{D^{(2)}(\varepsilon)}=\left\{\mathbf{z} \in D^{(2)}\right.$ : $\left.g_{D}(z, \infty) \geqslant \varepsilon\right\}:$

$$
\begin{aligned}
\left(-Q_{n} \psi_{n}\right)(z) & =\frac{1}{2 \pi i} \int_{\Gamma^{+}}\left(R_{n} \psi_{n} w\right)(\zeta) \frac{1}{\rho(\zeta)} d \Omega\left(z^{(2)} ; \zeta^{(2)}\right)-p_{n}(z) \\
& =\frac{1}{2 \pi i} \int_{\Gamma^{+}}\left(R_{n} \varphi_{n} w\right)(\zeta) \frac{\psi_{n}(\zeta)}{\varphi_{n}(\zeta)} \frac{1}{\rho(\zeta)} d \Omega\left(z^{(2)} ; \zeta^{(2)}\right)-p_{n}(z), \quad z \in \overline{D(\varepsilon)}
\end{aligned}
$$

Из (2.42) и условия выбора $\varepsilon_{n}$ вытекает, что

$$
\begin{aligned}
\max _{z \in L\left(\varepsilon_{n}\right)}\left|\left(Q_{n} \psi_{n}\right)(z)-p_{n}(z)\right| & =\max _{z \in L\left(\varepsilon_{n}\right)}\left|p_{n}(z)\right| \cdot O\left(q^{n}\right) \\
& =\min _{z \in L\left(\varepsilon_{n}\right)}\left|p_{n}(z)\right| \cdot O\left(q^{n}\right),
\end{aligned}
$$

где $0<q<1$. Поскольку мы заранее выбрали $\varepsilon>0$ так, что все g "свободных" нулей функции $\psi_{n}$ компактно (при $n \rightarrow \infty, n \in \Lambda$ ) принадлежат области $D(\varepsilon)$, то с помошью теоремы Руше легко получаем из $(2.43)$, что функции $\left(Q_{n} \psi_{n}\right)(z)$ и $p_{n}(z)$ для всех достаточно больших $n \in \Lambda$ имеют в $D\left(\varepsilon_{n}\right)$ одинаковое количество нулей. Так как функщия $\psi_{n}$ имеет в конечной плоскости ровно g нулей, получаем, что $\operatorname{deg} p_{n}=\mathrm{g}$ и все его g нулей компактно (по $n \in \Lambda$ ) принадлежат $D(\varepsilon)$. Отсюда уже вытекает: $\operatorname{deg} Q_{n}=n$ (т.е. индекс $n$ - нормальньй), все предельные точки $(n-m)$ нулей полинома $Q_{n}(z), n \in \Lambda$, лежат на $S$, а асимптотическое поведение g нулей полинома $p_{n}$ - такое 
же, как g "свободных" нулей $\psi_{n}(z)$. Легко также видеть, что если $p_{n}(z)=k_{n} z^{\mathrm{g}}+\cdots$, то $p_{n}(z) /\left(k_{n} X_{\mathrm{g}, n}(z)\right)=1+o\left(q^{n}\right)$ при $n \rightarrow \infty$ равномерно на $L\left(\varepsilon_{n}\right)$. Используя только что обнаруженные свойства полинома $p_{n}$ и тождество $\varphi_{n}(z) \psi_{n}(z) \equiv C_{n} X_{\mathrm{g}, n}(z) r_{n}(z)$, из $(2.43)$ легко получаем, что если нормировать $Q_{n}$ условием $Q_{n}(z)=z^{n}+\cdots$, то

$$
\max _{z \in L(\varepsilon)}\left|\frac{Q_{n}(z)}{\varphi_{n}(z)} r_{n}(z)-\frac{p_{n}(z)}{k_{n} X_{\mathrm{g}, n}(z)}\right|=o\left(q^{n}\right), \quad n \rightarrow \infty, \quad n \in \Lambda .
$$

Следовательно,

$$
\max _{z \in \bar{D}(\varepsilon)}\left|\frac{Q_{n}(z)}{\varphi_{n}(z)} r_{n}(z)-1\right|=o\left(q^{n}\right), \quad n \rightarrow \infty, \quad n \in \Lambda .
$$

В частности, для $f=\hat{\rho}$ имеем: $Q_{n}(z)=\varphi_{n}(z)\left(1+o\left(q^{n}\right)\right), 0<q<1$, при $n \rightarrow \infty$, $n \in \Lambda$, равномерно по $z \in \overline{D(\varepsilon)}, \varepsilon>0$ произвольно мало, $q=q(\varepsilon)$.

Вернемся теперь к случаю $\mathbf{z} \in D^{(1)}$. Непосредственно из (2.41) получаем, что функция $\left(R_{n} \varphi_{n} w\right)(z)$ имеет в $D_{\varepsilon}$ ровно $g$ конечных нулей. Следовательно, этим свойством обладает и функция $R_{n}(z)$. Тем самым, для $n \in \Lambda$ аппроксимации Паде $[n / n]_{\widehat{\rho}}$ интерполируют функиию $\widehat{\rho}(z)$ дополнительно в g точках, компактнопо $n \in \Lambda$ принадлежащих $D$.

5.2. Случай, когда допустимый вес $\rho$ аналитичен на $S$ : асимптотическая формула для полиномов Паде на множестве $S$. Непосредственно из формулы (2.41) вытекает, что граничные значения функции $R_{n} \varphi_{n} w$ на $S$ удовлетворяют соотношению: $\left(R_{n} \varphi_{n} w\right)^{ \pm}(\zeta)=o\left(q^{n}\right) \cdot M_{n}+p_{n}(\zeta), 0<q<1$. Откуда, используя свойства полинома $p_{n}$, получаем, что $\left(R_{n} \varphi_{n} w\right)^{ \pm}(\zeta)=p_{n}(\zeta)\left(1+o\left(q^{n}\right)\right)=X_{\mathrm{g}, n}(\zeta)\left(1+o\left(q^{n}\right)\right)$, $\zeta \in S, n \rightarrow \infty$. Тем самым,

$$
\left(R_{n}\right)^{ \pm}(\zeta)=\frac{X_{\mathrm{g}, n}(\zeta)}{\left(\varphi_{n} w\right)^{ \pm}(\zeta)}\left(1+o\left(q^{n}\right)\right)=\frac{\psi^{ \pm}}{w^{ \pm} r_{n}}(\zeta)\left(1+o\left(q^{n}\right)\right) .
$$

Учитьвая формулу для скачка функции $R_{n}(z)$ на $S$, получаем отсюда

$$
Q_{n}(\zeta)=\frac{\left(\psi_{n}^{+}+\psi_{n}^{-}\right)(\zeta)}{\left(r_{n} \rho\right)(\zeta)}+o\left(q^{n}\right)=\frac{\left(\varphi_{n}^{+}+\varphi_{n}^{-}\right)(\zeta)}{r_{n}(\zeta)}+o\left(q^{n}\right)
$$

В частности, для $f=\widehat{\rho}$ имеем: $Q_{n}(\zeta)=\varphi_{n}^{+}(\zeta)+\varphi_{n}^{-}(\zeta)+o\left(q^{n}\right), \zeta \in S$.

5.3. Случай, когда допустимый вес $\rho$ удовлетворяет условию Гёльдера на $S$. При исследовании это случая мы в основном следуем традиционному подходу, изложенному, например, в [58; $\S 8$, с. 181-184], [35], и опираемся на стандартную технику, связанную с изучением свойств граничных значений интегралов типа Коши и коэффициентов Фурье (см., например, [70; §5], [71; гл. II, §4], а также [41]).

Стандартными методами теории сингулярных интегралов типа Коши доказывается следуюшая 
Лемма. Пусть заданы: Н-непрерывная на единичной окружности $|z|=1$ функиия $h(z)$ и последовательность функиий $\left\{F_{n}(z)\right\}, F_{n}(z) \in \mathscr{H}(1<|z|<R) \cap$ $C(1 \leqslant|z| \leqslant R)$. Тогда для функиий

$$
f_{n}(z)=\frac{1}{2 \pi i} \int_{|t|=1} \frac{F_{n}(t) h(t)}{t^{n}} \frac{d t}{t-z}, \quad|z|<1,
$$

имеем: радиальные граничные значения $f_{n}\left(e^{i \theta}\right)$ существуют и

$$
\max _{0 \leqslant \theta \leqslant 2 \pi}\left|f_{n}\left(e^{i \theta}\right)\right|=o(1) \cdot \max _{1 \leqslant|z| \leqslant R}\left|F_{n}(z)\right|, \quad n \rightarrow \infty,
$$

где o(1) $=O\left((\log n) / n^{\alpha}\right)$, если $h \in \mathrm{H}(\alpha ;|z|=1), 0<\alpha \leqslant 1$.

Используем вновь интегральное уравнение $(2.40)$. Дифференциал $d \Omega(\mathbf{z} ; \boldsymbol{\zeta})$ при $\boldsymbol{\zeta}=\zeta^{(1)}, \mathbf{z}=z^{(1)}$ имеет вид

$$
d \Omega\left(\zeta^{(1)} ; z^{(1)}\right)=\frac{1}{2} \frac{\sqrt{h(\zeta)}+\sqrt{h(z)}}{\zeta-z} \frac{d \zeta}{\sqrt{h(\zeta)}}
$$

Для (многозначной) отображающей функции $\Phi(z)=e^{G(z, \infty)}$ имеем

$$
d \Phi=\Phi d G(z, \infty)=\frac{\Phi(\zeta) p(\zeta) d \zeta}{\sqrt{h(\zeta)}}
$$

Следовательно, $d \zeta / \sqrt{h(\zeta)}=d \Phi /(\Phi(\zeta) p(\zeta))$, где, как известно, $p(\zeta) \neq 0$ на $S$. Рассмотрим произвольный контур $\Gamma_{j}, j=1, \ldots, \mathrm{g}+1$, кривой $\Gamma$. Нетрудно видеть, что если мы сделаем в соответствуюшем интеграле из (2.40) замену переменных $t=$ $\Phi^{1 / \omega_{j}(\infty)}$, то окажемся в условиях леммы. Дальнейший ход рассуждений подобен пाп. 5.1 и 5.2 .

5.4. Связь теоремы Наттолла-Рахманова и теоремы Абеля. В условиях теорем имеем: $\widehat{\rho}(z)=-\frac{1}{2} \rho(z) / \sqrt{h(z)}, z \notin S ;$ при некотором $m \in \mathbb{N}$ и каждом $n=0(\bmod m)$ функция $V_{n}(z)=\Phi^{n}(z)+\Phi^{-n}(z)$ определена и однозначна в $\mathbb{C} \backslash$ $\left\{e_{1}, \ldots, e_{2 \mathrm{~g}+2}\right\}$ и, кроме того, $V_{n}(z)=O\left(z^{n}\right)$ при $n \rightarrow \infty$. Отсюда вытекает, что $V_{n}$ - полином степени $n$; теперь уже нетрудно доказать, что $Q_{n}(z)=$ const $\cdot V_{n}(z)$, a $P_{n}(z)=$ const $\cdot \widehat{\rho}(z)\left(\Phi^{n}(z)-\Phi^{-n}(z)\right)$. Очевидно, что в условиях теоремы функция $\Phi^{m}(z)=\exp \left\{m G_{D}(z, \infty)\right\}-$ однозначная мероморфная функция на римановой поверхности $\Re$, т.е. $\Phi^{m} \in \mathbb{C}(z, w)$. Следовательно, $\Phi^{m}(z)=\tau_{1}(z)+\tau_{2}(z) w(\mathbf{z}), \tau_{1}, \tau_{2} \in$ $\mathbb{C}(z)$. Так как $d G_{D}(z, \infty)=p(z) d z / \sqrt{h(z)}, p \in \mathbb{C}[z], p(z)=z^{\mathrm{g}}+\cdots$, то

$$
\int_{e_{1}}^{z} \frac{p(\zeta)}{\sqrt{h(\zeta)}} d \zeta=\frac{1}{m} \log \left(\tau_{1}(z)+\tau_{2}(z) \sqrt{h(z)}\right)
$$

значит, $p(z)$ - искомый интегрирующий множитель для дифференциала $d z / \sqrt{h(z)}$. 


\section{Глава III. \\ Аппроксимации Паде ортогональных разложений}

Эта глава посвящена одному из наиболее естественных обобщений классической конструкции Паде - апроксимациям Паде ортогональных разложений, которые впервые возникли при решении некоторых прикладных задач в связи с необходимостью приближенного представления функций, заданных полиномиальньм разложением, за пределами канонической области сходимости этого разложения. Два эквивалентных в классическом случае определения аппроксимаций Паде степенного ряда в рассматриваемой ситуации приводят к различным рациональным функциям. В обзоре рассмотрены оба варианта обобщения классической конструкции Паде. Для таких аппроксимаций получены аналоги классической теоремы Монтессу де Болора, а также некоторых результатов А. А. Гончара и В.В. Вавилова, в том числе - обратного характера.

\section{§1. Основные понятия и результаты}

1.1. Пусть на отрезке $\Delta=[-1,1]$ задана положительная борелевская мера $d \sigma(x)$ такая, что $\sigma^{\prime}(x)=d \sigma / d x>0$ почти всюду на $\Delta$. Пусть $\left\{P_{k}(x, d \sigma)\right\}_{n=0}^{\infty}-$ соответствуюшая система полиномов с положительньми старшими коэффициентами, ортонормированных по мере $d \sigma(x)$ :

$$
\int_{\Delta} P_{j}(x) P_{k}(x) d \sigma(x)= \begin{cases}1, & j=k \\ 0, & j \neq k\end{cases}
$$

$P_{k}(x)=P_{k}(x ; \sigma)=\alpha_{k} x^{k}+\cdots, \alpha_{k}>0, k=0,1, \ldots$ Наиболее важные примеры ортогональных полиномов такого типа: полиномы Лежандра $(d \sigma(x) \equiv d x)$ и Чебышёва $\left(d \sigma(x)=d x / \pi \sqrt{1-x^{2}}\right)$.

Произвольной вешественной функции $f(x)$, суммируемой на отрезке $\Delta$ по мере $d \sigma(x)$, соответствует (формальный или сходящийся) ряд Фурье по системе $\left\{P_{k}(x)\right\}$ :

$$
f(x) \sim \sum_{k=0}^{\infty} A_{k} P_{k}(x), \quad A_{k}=A_{k}(f)=\int_{\Delta} f(x) P_{k}(x) d \sigma(x)=:\left(f, P_{k}\right) .
$$

Сушествуют [43], [44] следуюшие два варианта определения рациональных аппроксимаций ряда (3.1), аналогичных аппроксимациям Паде степенного ряда (1.1).

Для фиксированных неотрицательных целых чисел $n$ и $m$ аппроксимацией Паде типа $(n, m)$ ряда (3.1) (или обобщенной аппроксимацией Паде функиии $f$, соответствующей системе $\left.\left\{P_{k}(x)\right\}\right)$ назовем:

А) рациональную функцию $R_{n, m} \in \mathscr{R}_{n, m}$ такую, что $R_{n, m}=p / q$, где $p, q \in \mathbb{C}[z]$, $\operatorname{deg} p \leqslant n, \operatorname{deg} q \leqslant m, q \not \equiv 0$ и

$$
(q f-p)(x) \sim \sum_{k=n+m+1}^{\infty} a_{k}^{(n, m)} P_{k}(x)
$$


В) рациональную функцию $F_{n, m} \in \mathscr{R}_{n, m} \cap \mathscr{H}(\Delta)$ такую, что

$$
\left(f-F_{n, m}\right)(x) \sim \sum_{k=n+m+1}^{\infty} b_{k}^{(n, m)} P_{k}(x) .
$$

Для нахождения функции $R_{n, m}$ (точнее, полиномов $p$ и $q$ ) требуется решить систему из $m$ линейных однородных уравнений относительно $m+1$ неизвестных величин коэффициентов полинома $q$. Таким образом, аппроксимация Паде типа $(n, m)$ в смысле варианта A) всегда существует, нетрудно однако заметить, что она, вообще говоря, не единственна. Вместе с тем, непосредственно из (3.2) вытекает, что если любой полином $q$, удовлетворяюший $(3.2)$, имеет степень ровно $m$, то рациональная функция $R_{n, m}$ определена однозначно: $R_{n, m}=R_{n, m}(f)$.

Для сушествования функщии $F_{n, m}$ требуется, чтобы сушествовало решение вытекающей непосредственно из определения нелинейной системы из $n+m+1$ уравнения относительно $n+m+2$ неизвестных величин, что оказьвается возможным не всегда. Тем самым, аппроксимация Паде типа $(n, m)$ ряда $(3.1)$ в смысле варианта В) (“нелинейная" аппроксимация Паде) определена не всегда. Однако если функция $F_{n, m}$ сушествует, то она единственна.

В отличие от классического случая указанные варианты определения приводят к различньм рациональным конструкциям. Это вытекает из того, что при фиксированных $n, m$ для построения рациональной функции $R_{n, m}$ требуется знать $n+2 m+1$ коэффициентов ряда (3.1), а для построения функции $F_{n, m}-$ так же, как и в классическом случае, $n+m+1$ коэффициентов. Кроме того, можно, например, подобрать последовательность чисел $\left\{A_{k}\right\}$ так, что нелинейные апшроксимации Паде $F_{n, 1}(z)$ типа $(n, 1)$ функции

$$
f(z)=\frac{1}{z-2}+\sum_{k=0}^{\infty} A_{k} T_{k}(z),
$$

где $T_{k}(z)$ - полиномы Чебьшёва первого рода, ортонормированные на $\Delta=[-1,1] \mathrm{c}$ весом $1 /\left(\pi \sqrt{1-x^{2}}\right)$, всегда существуют и не совпадают с соответствуюшими аппроксимациями $R_{n, 1}$ ни при каких $n$.

Подчеркнем еше раз, что ранее такие аппроксимации рассматривались только лишь как формальные конструкции, позволяюшие достаточно хорошо решать задачу численного приближения и представления заданной полиномиальным разложением в ряд (3.1) функции за пределами канонической области сходимости этого ряда. Вопросы сходимости этих рациональных апшроксимаций впервые рассмотрены автором обзора.

Более точно, речь идет об исследовании сходимости аналогов строчныл последовательностей рациональных аппроксимаций ортогональных разложений, прежде всего - об обобщении на каждый из рассмотренных выше случаев классической теоремы Монтессу де Болора.

Для любого $\rho>1$ положим $\Gamma_{\rho}=\{z \in \mathbb{C}:|\varphi(z)|=\rho\}$, где $\varphi$ - функция, конформно отображающая внешность отрезка $\Delta=[-1,1]$ на внешность единичного круга так, что $\varphi(\infty)=\infty, \varphi^{\prime}(\infty)>0\left(\varphi(z)=z+\sqrt{z^{2}-1}\right.$, где ветвь корня выбрана так, что $|\varphi(z)|>1$ вне $\Delta ; \Gamma_{\rho}$ - эллипс с фокусами в точках \pm 1 и суммой полуосей, равной $\left.\rho\right)$. 
Внутренность эллипса $\Gamma_{\rho}$ обозначим через $D_{\rho}$; области $D_{\rho}, \rho \in(1,+\infty)$, будем называть каноническими (относительно $\Delta$ ). Функция $f$ предполагается голоморфной на $\Delta$ (тем самым, и в некоторой окрестности $\Delta ; f \in \mathscr{H}(\Delta))$. Пусть $D_{0}(f)$ - максимальная каноническая область, в которую $f$ продолжается как голоморфная функция. Тогда $f$ может быть разложена в ряд Фурье (3.1) по системе $\left\{P_{k}(x)\right\}$, равномерно сходящийся к $f$ внутри (на компактных подмножествах) $D_{0}(f)$, при этом скорость сходимости характеризуется неравенством:

$$
\varlimsup_{n \rightarrow \infty}\left|f(z)-\sum_{k=0}^{n} A_{k} P_{k}(z)\right|^{1 / n} \leqslant \frac{|\varphi(z)|}{\rho_{0}}<1 .
$$

Аналогично, для любого $m \in \mathbb{N}$ через $D_{m}(f)$ обозначим максимальную каноническую область, в которую $f$ продолжается как мероморфная функция, число полюсов которой $\leqslant m$; имеем $D_{m}(f):|\varphi(z)|<\rho_{m}, \rho_{m}=\rho_{m}(f)$.

Основньми результатами третьей главы являются следующие теоремы 5 и 6 , обобщаюшие классический результат Монтессу де Болора.

Теорема 5. Если функиия $f$ имеет в области $D_{m}(f)$ ровно $m$ полюсов, то

$$
R_{n, m} \rightarrow f, \quad n \rightarrow \infty
$$

равномерно в сферической метрике внутри $D_{m}(f)$.

Тем самым, при достаточно больших $n$ рациональные функции $R_{n, m}$ имеют ровно $m$ конечных полюсов (т.е. полином $q=q_{n, m}(f)$ имеет степень ровно $m$ ); при $n \rightarrow \infty$ конечные полюсы $R_{n, m}$ стремятся к полюсам $f$ в $D_{m}(f)$, причем каждый полюс $f$ “притягивает” столько полюсов $R_{n, m}$, какова его кратность; в условиях теоремы 5 для достаточно больших $n$ сушествует единственная аппроксимация Паде $R_{n, m}=$ $R_{n, m}(f)$ функции $f$. Скорость сходимости последовательности $\left\{R_{n, m}\right\}$ к $f$ характеризуется неравенством

$$
\varlimsup_{n \rightarrow \infty}\left|f(z)-R_{n, m}(z)\right|^{1 / n} \leqslant \frac{|\varphi(z)|}{\rho_{m}}<1, \quad z \in D_{m}(f) .
$$

Теорема 6. Если функиия $f$ имеет ровно $m$ полюсов в области $D_{m}(f)$, то при каждом достаточно большом $n$ существует обобщенная аппроксимация Паде $F_{n, m}$ типа $(n, m)$ функиии $f u$

$$
F_{n, m} \rightarrow f, \quad n \rightarrow \infty
$$

равномерно в сферической метрике внутри $D_{m}(f)$.

В условиях теоремы 6 скорость сходимости последовательности $\left\{F_{n, m}\right\}$ к $f$ в $D_{m}(f)$ характеризуется неравенством

$$
\varlimsup_{n \rightarrow \infty}\left|f(z)-F_{n, m}(z)\right|^{1 / n} \leqslant \frac{|\varphi(z)|}{\rho_{m}}<1, \quad z \in D_{m}(f),
$$

аналогичным (3.3). 
Теоремы 5 и 6 являются аналогами теоремы Монтессу де Болора о сходимости классических аппроксимаций Паде в кругах мероморфности функции, заданной своим разложением в степенной ряд. Различные обобщения этой теоремы Монтессу де Болора на случай интерполяционных последовательностей рациональных функций с конечным числом свободных полюсов содержатся в работах Саффа [72] и А. А. Гончара [73], [16]. Ниже при доказательстве теоремы 5 мы будем придерживаться схемы рассуждений работы [73]. В соответствии с этим мы сначала докажем более общий результат о сходимости по емкости строчной последовательности апроксимаций Паде $\left\{R_{n, m}\right\}$ к функции $f$ при любом $m \in \mathbb{N}$ (см. предложение 3.1 ). Приводимая ниже в п. 1.2 лемма, вытекающая из результатов работы [73], показьвает, что теорема 5 является непосредственньм следствием этого более общего утверждения.

ПрЕДЛОЖЕНИЕ 3.1. При любом $m \in \mathbb{N}$ последовательность $\left\{R_{n, m}\right\}, n=$ $0,1,2, \ldots$, сходится по емкости к функиии $f$ равномерно внутри $D_{m}(f)$.

Из доказательства теорем 5 и 6 вытекает, что конечные полюсы рациональных функций $R_{n, m}$ и $F_{n, m}$ стремятся к полюсам $f$ в $D_{m}(f)$ со скоростью геометрической прогрессии.

Тем самым, следуюшее утверждение является обратным к теореме 5.

ПРЕДЛОЖЕНИЕ 3.2. Пусть для некоторого $m \in \mathbb{N}$ и всех достаточно больших $n$ аппроксимачии Паде $R_{n, m}$ ряда (3.1) имеют ровно $m$ конечных полюсов (т.е. полиномы $p$ и $q$ не имеют обших нулей и степень полинома $q=q_{n, m}$ равна в точности $m$ ) и существует полином $\omega_{m}(z)=\prod_{j=1}^{m}\left(z-z_{j}\right), z_{j} \notin \Delta$, такой, что при нормировке $q_{n, m}(z)=z^{m}+\cdots$ выполняется соотношение

$$
\varlimsup_{n \rightarrow \infty}\left\|\omega_{m}-q_{n, m}\right\|^{1 / n} \leqslant \delta<1
$$

Тогда

$$
\rho_{m}(f) \geqslant \frac{1}{\delta} \max _{1 \leqslant j \leqslant m}\left|\varphi\left(z_{j}\right)\right|
$$

и в области $D_{m}(f)=D_{\rho_{m}}, \rho_{m}=\rho_{m}(f)$, функиия $f$ имеет ровно $m$ полюсов (в точках $z_{1}, \ldots, z_{m}$ ).

Объединив прямое и обратное утверждения, получим

ПрЕДЛОЖЕНИЕ 3.3. Для функции $f$, голоморфной на $\Delta$, следующие два утверждения әквивалентны:

$1^{\circ}$. Функиия $f$ имеет в области $D_{m}(f)$ ровно т полюсов.

$2^{\circ}$. Рациональные функиии $R_{n, m}$ для всех достаточно больших $n$ имеют ровно $m$ конечных полюсов, и существует полином $\omega_{m}(z)=z^{m}+\cdots$ степени $m$ такой, что при нормировке $q_{n, m}(z)=z^{m}+\cdots$

$$
\varlimsup_{n \rightarrow \infty}\left\|\omega_{m}-q_{n, m}\right\|^{1 / n}=\delta<1 .
$$

\footnotetext{
${ }^{5}$ Под $\|\cdot\|$ здесь понимается норма соответствующего полинома в пространстве коэффиццентов.
} 
При этом (если выполнено какое-либо из условий $1^{\circ}$ и $2^{\circ}$ ) полюсы функиии $f \boldsymbol{\theta}$ $D_{m}(f)$ совпадают с нулями $z_{1}, \ldots, z_{m}$ полинома $\omega_{m} u$

$$
\rho_{m}(f)=\frac{1}{\delta} \max _{1 \leqslant j \leqslant m}\left|\varphi\left(z_{j}\right)\right|
$$

При доказательстве этих результатов используются некоторые асимптотические свойства ортогональных полиномов и функций второго рода в дополнении к отрезку $\Delta$. Из результатов работ Е. А. Рахманова [74], [75] следует, что все требуемые свойства имеют место при указанном выше условии на $d \sigma: \sigma^{\prime}(x)=d \sigma / d x>0$ почти всюду на $\Delta$. Хорошо известно [76], что нужные асимптотические свойства выполняются для полиномов Якоби и, в том числе, - для полиномов Чебышёва и Лежандра. Следовательно, теоремы 5 и 6 справедливы, в частности, и в случае, когда рациональные аппроксимации строятся для разложений в ряды Фурье по указанным классическим ортогональным полиномам.

1.2. Приведем некоторые определения и вспомогательные результаты, используемые в дальнейшем.

Пусть заданы: $\Omega$ - открытое множество в комплексной плоскости $\mathbb{C}$ и $\left\{\psi_{n}(z)\right\}-$ последовательность мероморфных в $\Omega$ функций.

ОПРЕДЕЛЕНИЕ 1. Будем говорить, что последовательность мероморфных функций $\left\{\psi_{n}(z)\right\}$ сходится равномерно внутри $\Omega$ к функции $\psi(z): \Omega \rightarrow \mathbb{C}$, если для любого компакта $K \subset \Omega$ эта последовательность равномерно сходится к $\psi$ на $K$ :

$$
\lim _{n \rightarrow \infty}\left\|\psi-\psi_{n}\right\|_{K}=0
$$

где $\|\cdot\|_{K}-$ sup-норма на $K$.

Из равномерной сходимости последовательности мероморфных функций на $K$ вытекает, что все функции этой последовательности, начиная с некоторой, не имеют полюсов на $K: \psi_{n} \in \mathscr{H}(K)$ при всех $n \geqslant n_{0}=n_{0}(K)$. Тем самым, предельная функция $\psi$ голоморфна на $K$. Из равномерной сходимости внутри открытого множества $\Omega$ вытекает, что полюсы функций $\left\{\psi_{n}\right\}$ не могут накапливаться внутри $\Omega$, т.е. их предельные точки лежат на гранище $\Omega$. В этом случае предельная функция $\psi$ голоморфна в $\Omega$. Такую равномерную сходимость будем обозначать символом “马”.

ОПРЕДЕЛЕНИЕ 2. Будем говорить, что последовательность мероморфных функций $\left\{\psi_{n}(z)\right\}$ сходится равномерно в сферической метрике внутри $\Omega$ к функции $\psi(z): \Omega \rightarrow \overline{\mathbb{C}}$, если для любого компакта $K \subset \Omega$ эта последовательность равномерно сходится в сферической метрике к $\psi$ на $K$ :

$$
\lim _{n \rightarrow \infty} \sup _{z \in K}\left|\psi(z) ; \psi_{n}(z)\right|=0
$$

где $|\cdot ; \cdot|_{K}-$ расстояние между точками в сферической метрике.

Из равномерной сходимости в сферической метрике на $K$ последовательности мероморфных функций вытекает, что все функции этой последовательности, начиная с некоторой, имеют одинаковое число полюсов на $K$; тем самым, предельная функция $\psi$ 
мероморфна на $K$ и имеет то же самое число полюсов. Следовательно, если $\psi$ голоморфна в $\Omega$, то предельные точки полюсов мероморфных функций $\left\{\psi_{n}(z)\right\}$ лежат на гранище $\Omega$.

Под емкостью сар $е$ произвольного множества $e \subset \mathbb{C}$ будем понимать его внешнюю емкость; в случае, если $e$ - компакт в $\mathbb{C}$, емкость $е$ совпадает с его трансфинитнылм диаметром.

ОПРЕДЕЛЕНИЕ 3. Будем говорить, что последовательность мероморфных функций $\left\{\psi_{n}\right\}$ сходится по емкости равномерно внутри $\Omega$ (к функции $\left.\psi: \Omega \rightarrow \overline{\mathbb{C}}\right)$, если для любых компакта $K \subset \Omega$ и числа $\varepsilon>0$ эта последовательность равномерно сходится к $\psi$ на множестве вида $K \backslash e_{\varepsilon}$, где сар $e_{\varepsilon}<\varepsilon$. Такую сходимость будем обозначать символом " $\stackrel{\text { cap }}{\rightrightarrows}$ ".

Непосредственно из леммы 1 работы [73] вытекает следующая

ЛЕмма. Пусть $\psi_{n} \stackrel{\text { сар }}{\rightrightarrows} \psi$ внутри $\Omega$. Тогда

а) если функиии $\psi_{n}$ голоморфны в $\Omega$, то последовательность $\left\{\psi_{n}\right\}$ равномерно сходится внутри $\Omega$ и, тем самым, $\psi$ голоморфна в $\Omega$;

b) если число полюсов $\psi_{n}$ в $\Omega$ не превосходит $m$, то $\psi$ мероморфна в $\Omega$ и число ее полюсов (в $\Omega)$ также не превосходит $m$; отсюда, в частности, следует, что если $\psi$ имеет полюс кратности $\nu$ в точке $а \in \Omega$, то по крайней мере $\nu$ полюсов функиий $\psi_{n}$ стремятся $\kappa$ точке а при $n \rightarrow \infty$ (другими словами, каждый полюс $\psi$ в $\Omega$ притягивает по крайней мере столько полюсов функций $\psi_{n}$, какова его кратность);

с) если функции $\psi_{n}$ имеют не более чем $m$ полюсов в $\Omega$, а $\psi$ имеет ровно $m$ полюсов, то для достаточно больших $n$ число полюсов $\psi_{n}$ в $\Omega$ так-

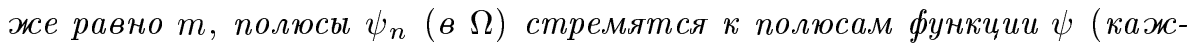
дый полюс $\psi$ притягивает столько полюсов $\psi_{n}$, какова его кратность) $u$ $\psi_{n} \rightrightarrows \psi$ внутри множества $\Omega^{\prime}$, получающегося из $\Omega$ удалением полюсов функиии $\psi$.

\section{§ 2. Доказательство предложения 3.1}

2.1. Пусть $\left\{H_{k}(z)\right\}$ - функции второго рода, соответствуюшие ортогональным полиномам $\left\{P_{k}(x)\right\}$, т.е.

$$
H_{k}(z)=\int_{-1}^{1} \frac{P_{k}(x) d \sigma(x)}{z-x}, \quad z \in \overline{\mathbb{C}} \backslash \Delta .
$$

Из результатов работ [75], [76] вытекает, что при рассматриваемых ограничениях на меру $\sigma\left(\sigma^{\prime}>0\right.$ почти всюду на $\left.\Delta\right)$ равномерно внутри области $D=\mathbb{C} \backslash \Delta$ вьполняются предельные соотношения

$$
\begin{aligned}
& \lim _{k \rightarrow \infty} \frac{P_{k+\ell}(z)}{P_{k}(z)}=\varphi(z)^{\ell}, \\
& \lim _{k \rightarrow \infty} \frac{H_{k+\ell}(z)}{H_{k}(z)}=\frac{1}{\varphi(z)^{\ell}}
\end{aligned}
$$


где $\ell$ - произвольное фиксированное целое число. Из формул (3.4) и (3.5) следуют равенства

$$
\begin{aligned}
\lim _{k \rightarrow \infty}\left|P_{k+\ell}(z)\right|^{1 / k} & =|\varphi(z)|, \\
\lim _{k \rightarrow \infty}\left|H_{k+\ell}(z)\right|^{1 / k} & =\frac{1}{|\varphi(z)|},
\end{aligned}
$$

в которых сходимость также равномерная внутри области $D$. Следовательно, разложение

$$
\frac{1}{t-z}=\sum_{k=0}^{\infty} H_{k}(t) P_{k}(z)
$$

сходится равномерно для $t \in \Gamma_{\rho}=\{t \in \mathbb{C}:|\varphi(t)|=\rho\}, \rho>1$, и для $z$ - на произвольном компакте, внутреннем к $\Gamma_{\rho}$. Отсюда вытекает, что ряды $(3.1)$ и (3.2) сходятся равномерно внутри области $D_{0}(f)$ соответственно к функциям $f$ и $q f-p$.

2.2. Приступим теперь непосредственно к доказательству предложения 3.1. Обозначим через $z_{1}, z_{2}, \ldots, z_{N}$ полюсы функции $f$ в области $D_{m}(f)$, а через $m_{1}, m_{2}, \ldots$, $m_{N}$ - их кратности; тогда $\sum_{j=1}^{N} m_{j}=\mu \leqslant m$. Положим

$$
\omega_{\mu}(z)=\prod_{j=1}^{N}\left(z-z_{j}\right)^{m_{j}}
$$

Фиксируем целое $n \geqslant 0$. Пусть $R_{n, m}=p_{n, m} / q_{n, m}$, где $p_{n, m}$ и $q_{n, m}$ взаимно просты, аппроксимация Паде типа $(n, m)$ ряда $(3.1)$, и пусть $t_{n, 1}, t_{n, 2}, \ldots, t_{n, \lambda}, \lambda=\lambda(n) \leqslant m,-$ нули полинома $q_{n, m}$, т.е. $q_{n, m}(z)=\prod_{j=1}^{\lambda}\left(z-t_{n, 1}\right)$. Ряд в правой части равенства (3.2) сходится равномерно внутри области $D_{0}(f)$, поэтому, после умножения обеих частей $(3.2)$ на $\omega_{\mu}(z)$, для $z \in D_{0}(f)$ получим

$$
\left(q_{n, m} f \omega_{\mu}-p_{n, m} \omega_{\mu}\right)(z)=\sum_{k=n+m+1}^{\infty} A_{k} \omega_{\mu}(z) P_{k}(z)
$$

Правую часть этого равенства разложим в ряд Фурье по системе $\left\{P_{\nu}\right\}$. Тогда для коэффициентов Фурье находим

$$
b_{\nu}=\sum_{k=n+m+1}^{\infty} A_{k}\left(\omega_{\mu} P_{k}, P_{\nu}\right)=\sum_{k=n+m+1}^{\infty} A_{k}\left(P_{k}, \omega_{\mu} P_{\nu}\right), \quad \nu=0,1,2, \ldots
$$

Из условия ортогональности полиномов $\left\{P_{k}\right\}$ следует, что $\left(\omega_{\mu} P_{\nu}, P_{k}\right)=0$ при $\mu+\nu<k$. Поэтому для коэффициентов $\left\{b_{\nu}\right\}$, учитьвая, что $\mu \leqslant m$, имеем $b_{\nu}=0$, $\nu=0,1, \ldots, n$, и

$$
b_{\nu}=\sum_{k=n+m+1}^{\nu+m} A_{k}\left(\omega_{\mu} P_{\nu}, P_{k}\right), \quad \nu=n+1, n+2, \ldots
$$


Тогда соотношение (3.8) можно представить в виде

$$
\left(q_{n, m} f \omega_{\mu}-p_{n, m} \omega_{\mu}\right)(z)=\sum_{k=n+1}^{n+m} b_{\nu} P_{\nu}(z)+\sum_{k=n+m+1}^{\infty} b_{\nu} P_{\nu}(z),
$$

где $z \in D_{m}(f)$, а $\left\{b_{\nu}\right\}$ определяются по формулам (3.9). Так как $\mu \leqslant m$, то $\operatorname{deg} \omega_{\mu} p_{n, m}$ $\leqslant n+m$. Следовательно, вторая сумма в правой части (3.10) представляет собой остаточньй член разложения функции $q_{n, m} f \omega_{\mu}$, голоморфной в области $D_{m}(f)$, в ряд Фурье по системе $\left\{P_{\nu}\right\}$. Поэтому для коэффициентов разложения имеем:

$$
b_{\nu}=\frac{1}{2 \pi i} \int_{\Gamma_{R}} f(t) \omega_{\mu}(t) q_{n, m}(t) H_{\nu}(t) d t, \quad \nu=n+m+1, n+m+2, \ldots
$$

где $R=\rho_{m}(f)-\delta$, а число $\delta>0$ и достаточно малое (его мы зафиксируем позднее) такое, что $R>\max _{1 \leqslant j \leqslant N}\left|\varphi\left(z_{j}\right)\right|$.

2.3. Рассмотрим первую сумму в правой части (3.10). В этом пункте мы получим некоторые оценки для модулей величин $b_{\nu}, \nu=n+1, n+2, \ldots, n+m$, через модули интегралов вида (3.11). Дальнейшее доказательство будет основано именно на этих оценках.

Прежде всего, в силу неравенства Коши-Буняковского и ортонормированности системы $\left\{P_{\nu}\right\}$ имеем:

$$
\left(\omega_{\mu} P_{\nu}, P_{k}\right)^{2} \leqslant\left(\omega_{\mu} P_{\nu}, \omega_{\mu} P_{\nu}\right) \cdot\left(P_{k}, P_{k}\right) \leqslant \max _{-1 \leqslant x \leqslant 1}\left|\omega_{\mu}(x)\right|^{2}, \quad k, \nu=0,1,2, \ldots
$$

Следовательно, при любых целых $k, \nu \geqslant 0$ справедливо неравенство

$$
\left|\left(\omega_{\mu} P_{\nu}, P_{k}\right)\right| \leqslant c_{1}
$$

где $c_{1}$ не зависит от $k, \nu$ и от $n$ (в дальнейшем через $c_{2}, c_{3}, \ldots$ обозначаются постоянные, также не зависяшие от $n)$.

Далее, функция $q_{n, m} f$ голоморфна в области $D_{0}(f)$. Поэтому для коэффициентов разложения $q_{n, m} f$ в ряд Фурье по системе $\left\{P_{k}\right\}$ имеем:

$$
A_{k}=\frac{1}{2 \pi i} \int_{\Gamma_{r}} q_{n, m}(t) f(t) H_{k}(t) d t, \quad k=0,1,2, \ldots, \quad r \in\left(1, \rho_{0}(f)\right) .
$$

При любом $k \geqslant 0$ функция $q_{n, m} f H_{k}$ мероморфнав замкнутой области $\bar{G}=\bar{D}_{R} \backslash D_{r}=$ $\{z \in \mathbb{C}: r \leqslant|\varphi(z)| \leqslant R\}$ и в точках $z_{1}, z_{2}, \ldots, z_{N}$ имеет полюсы, кратность которых не вьше, чем $m_{1}, m_{2}, \ldots, m_{N}$ соответственно. Теорема Коши о вычетах, примененная к функциям $q_{n, m} f H_{k}, k=0,1,2, \ldots$, в замкнутой области $\bar{G}$, приводит к равенствам

$$
\begin{gathered}
\frac{1}{2 \pi i} \int_{\Gamma_{R}} q_{n, m}(t) f(t) H_{k}(t) d t-\frac{1}{2 \pi i} \int_{\Gamma_{r}} d(t) f(t) H_{k}(t) d t=\sum_{j=1}^{N} \underset{z_{j}}{\operatorname{res}}\left(q_{n, m} f H_{k}\right), \\
k=0,1,2, \ldots
\end{gathered}
$$


Поскольку кратность полюса функции $q_{n, m} f H_{k}$ в точке $z_{j}$ не выше $m_{j}$, то по известной формуле для вычисления вычетов имеем:

$$
\underset{z_{j}}{\operatorname{res}}\left(q_{n, m} f H_{k}\right)=\frac{1}{\left(m_{j}-1\right) !} \lim _{z \rightarrow z_{j}}\left(\left(z-z_{j}\right)^{m_{j}} q_{n, m}(z) f(z) H_{k}(z)\right)^{\left(m_{j}-1\right)} .
$$

Используя формулу Лейбница и учитьвая, что $H_{n}(z) \neq 0$ при всех достаточно больших $n$ и $z \in \mathbb{C} \backslash \Delta$, преобразуем выражение, стоящее под знаком предела:

$$
\begin{aligned}
\left(\left(z-z_{j}\right)^{m_{j}} q_{n, m}(z) f(z) H_{k}(z)\right)^{\left(m_{j}-1\right)} & \left(\left(z-z_{j}\right)^{m_{j}} q_{n, m}(z) f(z) H_{n}(z) \cdot \frac{H_{k}(z)}{H_{n}(z)}\right)^{\left(m_{j}-1\right)} \\
\quad= & \sum_{p=0}^{m_{j}-1} C_{m_{j}-1}^{p}\left(\left(z-z_{j}\right)^{m_{j}} q_{n, m}(z) f(z) H_{n}(z)\right)^{\left(m_{j}-1-p\right)}\left(\frac{H_{k}(z)}{H_{n}(z)}\right)^{(p)} .
\end{aligned}
$$

Введем теперь следующие обозначения:

$$
\begin{gathered}
\alpha(j, p)=\frac{1}{\left(m_{j}-1\right) !} C_{m_{j}-1}^{p} \lim _{z \rightarrow z_{j}}\left(\left(z-z_{j}\right)^{m_{j}} q_{n, m}(z) f(z) H_{n}(z)\right)^{\left(m_{j}-1-p\right)} \\
j=1,2, \ldots, N, \quad p=0,1, \ldots, m_{j}-1 .
\end{gathered}
$$

Тогда равенство (3.14) представится в виде

$$
\underset{z_{j}}{\operatorname{res}}\left(q_{n, m} f H_{k}\right)=\sum_{p=0}^{m_{j}-1} \alpha(j, p)\left(\frac{H_{k}}{H_{n}}\right)^{(p)}\left(z_{j}\right) .
$$

Положим

$$
\gamma_{k}=\frac{1}{2 \pi i} \int_{\Gamma_{R}} q_{n, m}(t) f(t) H_{k}(t) d t, \quad k=0,1,2, \ldots
$$

Тогда, с учетом (3.13) и (3.14), для коэффициентов Фурье $\left\{A_{k}\right\}$ функции $q_{n, m} f$ получим

$$
A_{k}=\gamma_{k}-\sum_{j=1}^{N}\left[\sum_{p=0}^{m_{j}-1} \alpha(j, p)\left(\frac{H_{k}}{H_{n}}\right)^{(p)}\left(z_{j}\right)\right], \quad k=0,1,2, \ldots .
$$

С другой стороны, на основании определения обобщенных аппроксимаций Паде $R_{n, m}$ функции $f$ (см. соотношение (3.2)) имеем $A_{k}=0, k=n+1, n+2, \ldots, n+m$. Следовательно, мы получаем $m$ соотношений, связьваюших $\mu \leqslant m$ величин $\alpha(j, p)$, $j=1,2, \ldots, N, p=0,1, \ldots, m_{j}-1$ (напомним, что $m_{1}+\cdots+m_{N}=\mu \leqslant m$ ). Выбирая $\mu$ первых из этих соотношений: $A_{k}=0, k=n+1, n+2, \ldots, n+\mu$, получим

$$
\sum_{j=1}^{N} \sum_{p=0}^{m_{j}-1} \alpha(j, p)\left(\frac{H_{k}}{H_{n}}\right)^{(p)}\left(z_{j}\right)=\gamma_{k}, \quad k=n+1, n+2, \ldots, n+\mu .
$$


Эти соотношения позволят нам выразить $\alpha(j, p)$ через $\left(H_{k} / H_{n}\right)^{(p)}\left(z_{j}\right)$ и $\gamma_{k}, k=n+1$, $n+2, \ldots, n+\mu$. В самом деле, рассмотрим формулы $(3.17)$ как систему $\mu$ уравнений относительно $\mu$ "неизвестных" $\alpha(j, p)$. Определитель этой системы имеет вид

$\Delta_{n}=\operatorname{det}\left\|\left(\frac{H_{n+l}}{H_{n}}\right)^{(p)}\left(z_{j}\right)\right\|, \quad p=0,1, \ldots, m_{j}-1, \quad l=1,2, \ldots, \mu, \quad j=1,2, \ldots, N$.

Положим $\psi(z)=1 / \varphi(z)$. Тогда, учитьвая соотношение (3.5), получим

$$
\lim _{n \rightarrow \infty} \Delta_{n}=\widetilde{\Delta}=\left|\begin{array}{cccc}
\psi\left(z_{j}\right) & \psi^{\prime}\left(z_{j}\right) & \ldots & \psi^{\left(m_{j}-1\right)}\left(z_{j}\right) \\
\psi^{2}\left(z_{j}\right) & \left(\psi^{2}\right)^{\prime}\left(z_{j}\right) & \ldots & \left(\psi^{2}\right)^{\left(m_{j}-1\right)}\left(z_{j}\right) \\
\ldots \ldots \ldots \ldots \ldots \ldots \ldots \ldots \ldots \ldots \ldots \ldots \ldots \ldots & \ldots \ldots \ldots \ldots \ldots \ldots \ldots
\end{array}\right|_{(j=1,2, \ldots, N)}
$$

Символ, стоящий в правой части этого равенства, означает определитель порядка $\mu$, в котором последовательно вьписываются указанные групшы столбцов при $j=1,2$, $\ldots, N$. Функция $\psi(z)=1 / \varphi(z)$, конформно отображающая внешность отрезка $[-1,1]$ на внутренность единичного круга, однолистна вне этого отрезка. Поэтому $\widetilde{\Delta} \neq 0$. Значит, для достаточно больших $n$ определители $\Delta_{n}$ удовлетворяют неравенству $\left|\Delta_{n}\right| \geqslant c_{2}>0$. Только такие $n$ мы и будем рассматривать в дальнейшем.

2.4. Пусть $\Delta_{n}(j, p)$ - определитель, полученный из $\Delta_{n}$ заменой столбца с номером $q=\sum_{l=1}^{j-1} m_{l}+p+1$ на столбец из $\gamma_{n+1}, \gamma_{n+2}, \ldots, \gamma_{n+\mu}$. Решая систему (3.17) относительно величин $\alpha(j, p)$, получим $\alpha(j, p)=\Delta_{n}(j, p) / \Delta_{n}$. Разложим определитель $\Delta_{n}(j, p)$ по $q$-му столбцу: $\Delta_{n}(j, p)=\sum_{s=1}^{\mu} \gamma_{n+s} M(s, q)$, где $M(s, q)$ - минор, дополнительный к элементу с номерами $s, q$. Тогда имеем $\alpha(j, p)=\frac{1}{\Delta_{n}} \sum_{s=1}^{\mu} \gamma_{n+s} M(s, q)$. Подставив это выражение в формулу (3.16) для коэффициентов $\left\{A_{k}\right\}$, получим

$$
A_{k}=\gamma_{k}-\frac{1}{\Delta_{n}} \sum_{j=1}^{N} \sum_{p=0}^{m_{j}-1} \sum_{s=1}^{\mu} \gamma_{n+s} M(s, q)\left(\frac{H_{k}}{H_{n}}\right)^{(p)}\left(z_{j}\right),
$$

где $k=n+m+1, \ldots, n+2 m$ (именно для этих номеров $k$ и будет использоваться эта формула).

Из соотношения (3.5) легко следует, что для всех достаточно больших $n$ и $p=$ $0,1, \ldots, m, l=1,2, \ldots, 2 m, j=1,2, \ldots, N$ справедливо неравенство

$$
\left|\left(H_{n+l} / H_{n}\right)^{(p)}\left(z_{j}\right)\right| \leqslant c_{3},
$$

из которого вытекает, что $|M(s, q)| \leqslant(\mu-1) ! c_{3}^{\mu-1}=c_{4}$, где $s, q=1,2, \ldots, \mu$. Объединяя полученные оценки и учитывая, что $\left|\Delta_{n}\right| \geqslant c_{2}>0$, из формулы (3.18) для $n \geqslant N_{1}$ и $k=n+m+1, \ldots, n+2 m$ получаем

$$
\left|A_{k}\right| \leqslant\left|\gamma_{k}\right|+\frac{1}{c_{2}} c_{3} c_{4} m \sum_{s=1}^{\mu}\left|\gamma_{n+s}\right| \leqslant c_{5} \sum_{s=1}^{2 m}\left|\gamma_{n+s}\right|
$$


Наконец, из формулы (3.9) с учетом (3.12) и (3.19) следует неравенство для коэффициентов $b_{\nu}, \nu=n+1, n+2, \ldots, n+m$,

$$
\left|b_{\nu}\right| \leqslant \sum_{k=n+m+1}^{\nu+m}\left|A_{k}\right| \cdot\left|\left(\omega_{\mu} P_{\nu}, P_{k}\right)\right| \leqslant c_{1} c_{5} m \sum_{s=1}^{2 m}\left|\gamma_{n+s}\right| \leqslant c_{6} \sum_{s=1}^{2 m}\left|\gamma_{n+s}\right|,
$$

справедливое при достаточно больших $n\left(n \geqslant N_{1}\right)$.

2.5. Пусть теперь $K$ - произвольньй компакт, принадлежаший области $D_{m}(f)$; $\varkappa>0$ - минимальное число такое, что $K \subset \bar{D}_{\varkappa}\left(1<\varkappa<\rho_{m}(f)\right)$. Как и выше, положим $R=\rho_{m}(f)-\delta$, где $\delta>0$ выбрано столь малым, что $R>\varkappa$ и область $D_{R}$ содержит все полюсы функции $f$; кроме того, потребуем, чтобы вьполнялось неравенство $(\varkappa+\delta) /(R-\delta)<1$ (смысл этого требования станет ясен позднее). Зафиксируем теперь число $\delta$, удовлетворяющее указанным условиям.

Разделим обе части равенства (3.10) на $q_{n, m}(z)$, получим

$$
\left(f \omega_{\mu}-R_{n, m} \omega_{\mu}\right)(z)=\sum_{\nu=n+1}^{n+m} \frac{b_{\nu}}{q_{n, m}(z)} P_{\nu}(z)+\sum_{\nu=n+m+1}^{\infty} \frac{b_{\nu}}{q_{n, m}(z)} P_{\nu}(z) .
$$

Разумеется, это равенство содержательно для $z \in \bar{D}_{\varkappa}$, отличных от нулей полинома $q_{n, m}$. Для $\nu=n+1, \ldots, n+m$ из (3.20) имеем:

$$
\left|\frac{b_{\nu}}{q_{n, m}(z)}\right| \leqslant c_{6} \sum_{s=1}^{2 m}\left|\frac{\gamma_{n+s}}{q_{n, m}(z)}\right| .
$$

В соответствии с обозначениями п. 2.3

$$
\frac{\gamma_{n+s}}{q_{n, m}(z)}=\frac{1}{2 \pi i} \int_{\Gamma_{R}} f(t) H_{n+s}(t) \frac{q_{n, m}(t)}{q_{n, m}(z)} d t .
$$

Оценим отдельно множители, стояшие под знаком интеграла при $f(t)$, для $t \in \Gamma_{R}$ и $z \in \bar{D}_{\varkappa}$. Для достаточно больших $n$ и $s=1,2, \ldots, 2 m$ из (3.7) следует неравенство

$$
\max _{t \in \Gamma_{R}}\left|H_{n+s}(t)\right| \leqslant \frac{1}{(R-\delta)^{n}} .
$$

Для оценки второго сомножителя поступим так же, как в работе [73]. Положим $q_{n, m}(z ; R)=\prod_{R}^{\prime}\left(z-t_{n, j}\right)$, где $\prod_{R}^{\prime}$ означает произведение по тем $j$, для которых $\left|\varphi\left(t_{n, j}\right)\right|<R$ (т.е. $\left.t_{n, j} \in D_{R}\right)$. Легко видеть, что $|(t-\zeta) /(z-\zeta)|<c_{7}$ для всех $t \in \Gamma_{R}$, $z \in \bar{D}_{\varkappa}$ и $\zeta$ таких, что $|\varphi(\zeta)| \geqslant R$. Поэтому

$$
\left|\frac{q_{n, m}(t)}{q_{n, m}(z)}\right| \leqslant c_{7}^{m}\left|\frac{q_{n, m}(t ; R)}{q_{n, m}(z ; R)}\right| \leqslant \frac{c_{8}}{\left|q_{n, m}(z ; R)\right|}, \quad z \in \bar{D}_{\varkappa}, \quad t \in \Gamma_{R} .
$$

Из формулы (3.23), учитывая (3.24) и (3.25) (и очевидным образом оценивая $f$ под знаком интеграла), получаем

$$
\left|\frac{\gamma_{n+s}}{q_{n, m}(z)}\right| \leqslant \frac{c_{9}}{\left|q_{n, m}(z ; R)\right|} \cdot \frac{1}{(R-\delta)^{n}}, \quad z \in \bar{D}_{\varkappa}, \quad s=1,2, \ldots, 2 m, \quad n \geqslant N_{2} .
$$


Отсюда и из неравенства (3.22) следует, что для $n \geqslant N_{3}$ имеем:

$$
\left|\frac{b_{\nu}}{q_{n, m}(z)}\right| \leqslant \frac{c_{10}}{\left|q_{n, m}(z ; R)\right|} \cdot \frac{1}{(R-\delta)^{n}}, \quad \nu=n+1, \ldots, n+m, \quad z \in \bar{D}_{\varkappa} .
$$

Аналогичные рассуждения, проведенные для $\nu \geqslant n+m+1$ (в этом случае надо воспользоваться формулой (3.11)), приводят к следующим оценкам:

$$
\left|\frac{b_{\nu}}{q_{n, m}(z)}\right| \leqslant \frac{c_{11}}{\left|q_{n, m}(z ; R)\right|} \cdot \frac{1}{(R-\delta)^{\nu}}, \quad n \geqslant N_{4}, \quad z \in \bar{D}_{\varkappa} .
$$

С другой стороны, из соотношений (3.6) и принщипа максимума модуля для голоморфных функций следует неравенство

$$
\max _{z \in \bar{D}_{\varkappa}}\left|P_{n+l}(z)\right| \leqslant(\varkappa+\delta)^{n}, \quad n \geqslant N_{5}, \quad l=0,1, \ldots, m .
$$

Объединяя оценки (3.26)-(3.28), из формулы (3.21) получаем

$$
\left|\left(f \omega_{\mu}-R_{n, m} \omega_{\mu}\right)(z)\right| \leqslant \frac{c_{10} m}{\left|q_{n, m}(z ; R)\right|}\left(\frac{\varkappa+\delta}{R-\delta}\right)^{n}+\frac{c_{11}}{\left|q_{n, m}(z ; R)\right|} \sum_{\nu=n+m+1}^{\infty}\left(\frac{\varkappa+\delta}{R-\delta}\right)^{\nu}
$$

где $z \in \bar{D}_{\varkappa}, n \geqslant N_{6}$. Напомним, что $\delta>0$ было выбрано так, что величина $\theta=(\varkappa+\delta) /(R-\delta)<1$. Следовательно, оценка $(3.29)$ приводится к виду

$$
\left|\left(f \omega_{\mu}-R_{n, m} \omega_{\mu}\right)(z)\right| \leqslant \frac{c_{12} \theta^{n}}{\left|q_{n, m}(z ; R)\right|} .
$$

Таким образом, имеем окончательное неравенство

$$
\left|\left(f-R_{n, m}\right)(z)\right| \leqslant \frac{c_{12} \theta^{n}}{\left|q_{n, m}(z ; R) \omega_{\mu}(z)\right|}, \quad z \in \bar{D}_{\varkappa},
$$

справедливое для достаточно больших $n\left(n \geqslant N_{6}\right)$.

Положим $\tau=\omega_{\mu} q_{n, m}$; старший коэффищиент полинома $\tau(z)$ равен 1 , а его степень не превосходит $\mu+m \leqslant 2 m$. Фиксируем произвольное число $v \in(\theta, 1)$, тогда $q=$ $\theta / v<1 ;$ из (3.30) вытекает, что

$$
e_{n}=\left\{z \in \bar{D}_{\varkappa}:\left|\left(f-R_{n, m}\right)(z)\right| \geqslant v^{n}\right\} \subset\left\{z \in \bar{D}_{\varkappa}:|\tau(z)| \leqslant c_{12} q^{n}\right\}=E_{n} .
$$

Используя монотонность емкости, а также следующее хорошо известное свойство (см., например, [77; с. 290]):

$$
\operatorname{cap}\left\{z \in \mathbb{C}:\left|z^{n}+a_{1} z^{n-1}+\cdots+a_{n}\right| \leqslant \rho^{n}\right\}=\rho, \quad \rho>0,
$$

для достаточно больших $n$ получаем

$$
\operatorname{cap} e_{n} \leqslant \operatorname{cap} E_{n} \leqslant\left(c_{12} q^{n}\right)^{1 /(2 m)}=c_{13} \delta^{n},
$$

где $\delta \in(q, 1)$. Отсюда уже легко получаем утверждение предложения 3.1. 
2.6. Полученное неравенство (3.30) можно использовать для распространения на аппроксимации Паде ортогональных разложений и некоторых других результатов, справедливых для классического случая степенного ряда.

Например, на такие обобшенные аппроксимашии Паде переносится основной результат работы В.В.Вавилова [78]. Аналог этого результата дополняет предложение 3.1 и формулируется следуюшим образом.

ПРЕДЛОЖЕНИЕ 3.4. Если при фиксированном $m \in \mathbb{N}$ последовательность $\left\{R_{n, m}\right\}, n=0,1,2, \ldots$, сходится по емкости в некоторой окрестности точки $a \in \mathbb{C}$, mo $a \in D_{m}(f)$ (m.e. $\left.|\varphi(a)|<\rho_{m}(f)\right)$.

Отсюда и из предложения 3.1 вытекает

ПРЕДЛОЖЕНИЕ 3.5. При фиксированном $m \in \mathbb{N}$ область $D_{m}(f)$ является максимальным открытым множеством, внутри которого сходится по емкости строчная последовательность $\left\{R_{n, m}\right\}_{n \in \mathbb{N}}$ аппроксимачий Паде ряда (3.1).

Доказательство этого утверждения проводится по схеме, изложенной в работе [78], и опирается на неравенство (3.30).

\section{§3. Доказательство теоремы 6}

3.1. Схема доказательства теоремы 6 сушественно отличается от доказательства аналогичного результата о сходимости последовательности аппроксимаций $\left\{R_{n, m}\right\}-$ теоремы 5. В частности, значительную трудность представляет доказательство $c y$ ществования в условиях теоремы 6 рациональных функций $F_{n, m}$ для достаточно больших $n$, в то время как функции $R_{n, m}$ сушествуют всегда.

Приведем сначала некоторые обозначения и результаты, необходимые в дальнейшем.

Для матрицы, состоящей из элементов $a_{k j}, k=1,2, \ldots, n, j=1,2, \ldots, m$, будем применять обозначение $\left\|a_{k j}\right\|_{1 \leqslant k \leqslant n, 1 \leqslant j \leqslant m}$, где $k$ означает номер строки, а $j$ - номер столбца, либо другое обозначение $\left\|a_{k 1}, \ldots, a_{k m}\right\|_{1 \leqslant k \leqslant n}$. Через

$$
\left\|a_{k j}, b_{k}\right\|_{1 \leqslant k \leqslant n, 1 \leqslant j \leqslant m} \text { и }\left\|a_{k j}, b_{k j}\right\|_{1 \leqslant k \leqslant n, 1 \leqslant j \leqslant m}
$$

обозначим соответственно матрицы

$$
\left\|a_{k 1}, \ldots, a_{k m}, b_{k}\right\|_{1 \leqslant k \leqslant n} \quad \text { и }\left\|a_{k 1}, b_{k 1}, \ldots, a_{k m}, b_{k m}\right\|_{1 \leqslant k \leqslant n} .
$$

В дальнейшем при записи матрицы (или соответствующего ей определителя) пределы, в которых изменяются индексы элементов, иногда будем опускать.

Пусть, как и в $\S 2,\left\{H_{k}(z)\right\}$ - функции второго рода, соответствующие ортогональным по мере $\sigma$ полиномам $\left\{P_{k}(x)\right\}$. Напомним, что функщия $H_{k}(z)$ голоморфнав точке $z=\infty$ и имеет в ней нуль порядка $k+1$, а из результатов работ [74], [75] следует, что при сформулированном в теореме 6 условии на меру $\sigma$ равномерно внутри области $\mathbb{C} \backslash \Delta$ имеют место асимптотические соотношения $(3.4),(3.5)$ из $\S 2$.

Для функции $f \in \mathscr{H}(\Delta)$ (тем самым, голоморфной и в некоторой окрестности $\Delta$ ) имеем

$$
A_{k}(f)=\int_{\Delta} f(x) P_{k}(x) d \sigma(x)=\frac{1}{2 \pi i} \int_{\Gamma_{\rho}} f(t) H_{k}(t) d t
$$


где $\rho>1$ выбрано так, что $f \in \mathscr{H}\left(\bar{D}_{\rho}\right)$. При наличии указанной в $\S 2$ асимптотики для ортогональных полиномов и функций второго рода для любой функции $f \in \mathscr{H}(\Delta)$ ее ряд Фурье по системе $\left\{P_{k}\right\}$ сходится к $f$ равномерно внутри (на компактных подмножествах) области $D_{0}(f)$ - максимальной канонической области голоморфности $f$. Поэтому если функция $F_{n, m}$ существует, то по определению

$$
\left(f-F_{n, m}\right)(z)=\sum_{k=n+m+1}^{\infty} b_{k}^{(n, m)} P_{k}(z), \quad z \in D_{0}\left(f-F_{n, m}\right) .
$$

3.2. Приступим теперь непосредственно к доказательству теоремы. Во избежание излишней громоздкости изложения мы ограничимся случаем, когда функция $f$ имеет $m$ различных полюсов $z_{1}, z_{2}, \ldots, z_{m}$ в области $D_{m}(f)$. Пользуясь техникой, развитой в $\S 2$ настоящей главы (см. (3.14)-(3.18)), можно легко сделать нижеследующие рассуждения пригодными и для общего случая кратных полюсов.

СХемА ДОКАЗАТЕЛЬСТВА ТЕОРЕмЫ 6 такова. Сначала мы докажем существование апшроксимации $F_{n, m}$ для всякого достаточно большого $n$. Одновременно будет установлена и асимптотика конечных полюсов функций $F_{n, m}$ при $n \rightarrow \infty$. Затем будут доказаны и остальные утверждения теоремы.

В основе доказательства лежит следующая

ЛЕмма. Пусть $\sigma$ - положительная борелевская мера, $S_{\sigma}=\Delta u \sigma^{\prime}>0$ почти всюду на $\Delta$. Если при фиксированных $\varkappa>1$ и $m \in \mathbb{N}$ функция $f$ мероморфна в области $D_{\varkappa}$ и имеет в ней ровно $m$ простых полюсов $z_{1}, z_{2}, \ldots, z_{m}$, то система 2 тр урвнений

$$
\sum_{j=1}^{m} x_{j} H_{n+k}\left(y_{j}\right)=A_{n+k}(f), \quad k=1, \ldots, 2 m,
$$

относительно $2 m$ неизвестных $x_{1}, x_{2}, \ldots, x_{m}, y_{1}, y_{2}, \ldots, y_{m}$ имеет (единственное) решение $x_{n, j}, y_{n, j}, j=1, \ldots, m$, такое, что

$$
\lim _{n \rightarrow \infty} y_{n, j}=z_{j}, \quad j=1, \ldots, m
$$

ДокАЗАТЕЛЬСТВо ЛЕмМЫ. Введем две матрицы

$$
\begin{gathered}
U_{n}\left(y_{1}, \ldots, y_{m}\right)=\left\|H_{n+k}\left(y_{j}\right)\right\|_{1 \leqslant k \leqslant 2 m, 1 \leqslant j \leqslant m}, \\
\widetilde{U}_{n}\left(y_{1}, \ldots, y_{m}\right)=\left\|H_{n+k}\left(y_{j}\right), A_{n+k}(f)\right\|_{1 \leqslant k \leqslant 2 m, 1 \leqslant j \leqslant m} .
\end{gathered}
$$

Сушествование решения системы (3.33) эквивалентно разрешимости относительно $y_{1}, y_{2}, \ldots, y_{m}$ уравнения

$$
\operatorname{rank} U_{n}\left(y_{1}, \ldots, y_{m}\right)=\operatorname{rank} \widetilde{U}_{n}\left(y_{1}, \ldots, y_{m}\right),
$$

в котором отсутствуют величины $x_{1}, x_{2}, \ldots, x_{m}$. 
Выберем теперь $\varepsilon>0$ такое, что множества $\left\{z \in \mathbb{C}:\left|z-z_{j}\right| \leqslant 2 \varepsilon\right\}, 1 \leqslant j \leqslant m$, не пересекаются между собой и с отрезком $\Delta$ (ниже на $\varepsilon$ будет наложено еще одно, дополнительное условие малости). Положим далее $E_{j}=\left\{z \in \mathbb{C}:\left|z-z_{j}\right| \leqslant \varepsilon\right\}$, $\gamma_{j}=\left\{z \in \mathbb{C}:\left|z-z_{j}\right|=2 \varepsilon\right\}, 1 \leqslant j \leqslant m$.

Решение $y_{n, 1}, y_{n, 2}, \ldots, y_{n, m}$ уравнения (3.34) будем искать только в замкнутых $\varepsilon$-окрестностях $E_{1}, E_{2}, \ldots, E_{m}$ точек $z_{1}, z_{2}, \ldots, z_{m}$. Таким образом, всюду в дальнейшем, не оговаривая этого специально, мы предполагаем, что “переменные" $y_{j} \in E_{j}, 1 \leqslant j \leqslant m$.

Положим $\psi_{n, k}(z)=H_{n+k}(z) / H_{n}(z)$, тогда соотношение (3.5) примет вид

$$
\psi_{n, k}(z) \rightrightarrows \psi^{k}(z), \quad z \in \mathbb{C} \backslash \Delta .
$$

Для каждого достаточно большого $n$ (и при условиях $y_{1} \in E_{1}, y_{2} \in E_{2}, \ldots$, $\left.y_{m} \in E_{m}\right)$ имеем

$$
\operatorname{rank} U_{n}\left(y_{1}, \ldots, y_{m}\right)=\operatorname{rank}\left\|\psi_{n, k}\left(y_{j}\right)\right\|_{1 \leqslant k \leqslant 2 m, 1 \leqslant j \leqslant m}
$$

Из (3.35) следует, что равномерно по $y_{j} \in E_{j}, 1 \leqslant j \leqslant m$, вьполняется предельное соотношение

$$
\lim _{n \rightarrow \infty} \operatorname{det}\left\|\psi_{n, k+s}\left(y_{j}\right)\right\|_{1 \leqslant k, j \leqslant m}=\operatorname{det}\left\|\psi^{k+s}\left(y_{j}\right)\right\|_{1 \leqslant k, j \leqslant m}, \quad 0 \leqslant s \leqslant m,
$$

причем определитель в правой части (3.36) отличен от нуля. Отсюда легко находим, что $\operatorname{rank} U\left(y_{1}, \ldots, y_{m}\right)=m$ для всех достаточно больших $n$. Поэтому уравнение (3.34) равносильно следующему уравнению:

$$
\operatorname{rank} \widetilde{U}\left(y_{1}, \ldots, y_{m}\right)=m
$$

Далее, так как $\operatorname{rank} U\left(y_{1}, \ldots, y_{m}\right)=m$, то $\operatorname{rank} \widetilde{U}\left(y_{1}, \ldots, y_{m}\right) \geqslant m . \quad$ Следовательно, соотношение (3.37) эквивалентно тому, что любые $m+1$ строк матрищы $\widetilde{U}_{n}\left(y_{1}, \ldots, y_{m}\right)$ линейно зависимы, т.е. любой минор порядка $m+1$ этой матрицы равен нулю. Но из соотношения (3.36) легко следует, что для обращения в нуль всех миноров порядка $m+1$ матрицы $\widetilde{U}_{n}\left(y_{1}, \ldots, y_{m}\right)$ необходимо и достаточно, чтобы обрашались в нуль лишш следуюшие $m$ из них:

$$
\operatorname{det}\left\|H_{n+k+s}\left(y_{p}\right), A_{n+k+s}(f)\right\|_{0 \leqslant k \leqslant m, 1 \leqslant p \leqslant m}=0, \quad 1 \leqslant s \leqslant m .
$$

Таким образом, уравнение (3.34) для всех достаточно больших $n$ и при условиях $y_{j} \in E_{j}, 1 \leqslant j \leqslant m$, эквивалентно системе $m$ уравнений (3.38) относительно $m$ величин $y_{1}, y_{2}, \ldots, y_{m}$.

По условиям леммы функция $f$ мероморфна в области $D_{\varkappa}$ и имеет там $m$ простых полюсов в точках $z_{1}, z_{2}, \ldots, z_{m}$. Следовательно, для $z \in D_{\varkappa}$ справедливо представление

$$
f(z)=-\sum_{j=1}^{m} \frac{d_{j}}{z-z_{j}}+g(z)
$$


где $d_{j} \neq 0$ и $g \in \mathscr{H}\left(D_{\varkappa}\right)$. Поэтому с учетом (3.31) имеем

$$
\begin{aligned}
A_{n}(f) & =-\sum_{j=1}^{m} d_{j} \cdot A_{n}\left(\frac{1}{z-z_{j}}\right)+A_{n}(g) \\
& =\sum_{j=1}^{m} d_{j} \cdot H_{n}\left(z_{j}\right)+A_{n}(g),
\end{aligned}
$$

где

$$
A_{n}(g)=\frac{1}{2 \pi i} \int_{\Gamma_{\rho}} g(t) H_{n}(t) d t, \quad \rho \in(1, \varkappa) .
$$

Из соотношений (3.31) следует, что $A_{n}(g)=o\left(\delta^{n}\right)$ при $n \rightarrow \infty$ для любого фиксированного $\delta \in(1 / \varkappa, 1)$.

В силу представления (3.39) для коэффициентов Фурье функщии $f$ система $(3.38)$ приводится к виду

$$
\begin{aligned}
& \sum_{j=1}^{m} d_{j} H_{n+s}\left(z_{j}\right) B_{n+s, j}\left(y_{1}, \ldots, y_{m}\right) \\
& \quad+\operatorname{det}\left\|\psi_{n+s, k}\left(y_{p}\right), A_{n+s+k}(g)\right\|_{0 \leqslant k \leqslant m, 1 \leqslant p \leqslant m}=0, \quad 1 \leqslant s \leqslant m
\end{aligned}
$$

где введено обозначение

$$
B_{n, j}\left(y_{1}, \ldots, y_{m}\right)=\operatorname{det}\left\|\psi_{n, k}\left(y_{p}\right), \psi_{n, k}\left(z_{j}\right)\right\|_{0 \leqslant k \leqslant m, 1 \leqslant p \leqslant m}
$$

Преобразуем теперь эти определители к виду, удобному для дальнейших рассуждений. Для $1 \leqslant j \leqslant m$ и $t \in E_{j}$ имеем

$$
\psi_{n, k}(t)=\sum_{l=0}^{\infty} \psi_{n, k}^{(l)}\left(z_{j}\right) \frac{\left(t-z_{j}\right)^{l}}{l !}
$$

и, следовательно, получаем

$$
\psi_{n, k}(t)-\psi_{n, k}\left(z_{j}\right)=\left(t-z_{j}\right) \psi_{n, k}^{\prime}\left(z_{j}\right)+\left(t-z_{j}\right)^{2} \theta_{n, k}\left(t, z_{j}\right),
$$

где

$$
\begin{aligned}
\theta_{n, k}\left(t, z_{j}\right) & =\sum_{l=2}^{\infty}\left(\psi_{n, k}\right)^{(l)}\left(z_{j}\right) \frac{\left(t-z_{j}\right)^{l-2}}{l !}=\sum_{l=2}^{\infty}\left(t-z_{j}\right)^{l-2} \frac{1}{2 \pi i} \int_{\gamma_{j}} \frac{\psi_{n, k}(u) d u}{\left(u-z_{j}\right)^{l+1}} \\
& \rightrightarrows \sum_{l=2}^{\infty}\left(t-z_{j}\right)^{l-2} \frac{1}{2 \pi i} \int_{\gamma_{j}} \frac{\psi^{k}(u) d u}{\left(u-z_{j}\right)^{l+1}} \\
& =\sum_{l=2}^{\infty}\left(\psi^{k}\right)^{(l)}\left(z_{j}\right) \frac{\left(t-z_{j}\right)^{l-2}}{l !}=\theta_{k}\left(t, z_{j}\right), \quad n \rightarrow \infty, \quad t \in E_{j} .
\end{aligned}
$$


Поэтому для указанных вьше определителей находим представление

$$
\begin{aligned}
\operatorname{det}\left\|\psi_{n, k}\left(y_{p}\right), \psi_{n, k}\left(z_{j}\right)\right\|_{0 \leqslant k \leqslant m, 1 \leqslant p \leqslant m} & \\
= & \operatorname{det}\left\|\psi_{n, k}\left(y_{p}\right), \psi_{n, k}\left(z_{j}\right)-\psi_{n, k}\left(y_{j}\right)\right\| \\
= & \left(z_{j}-y_{j}\right)\left(\operatorname{det}\left\|\psi_{n, k}\left(y_{p}\right),\left(\psi_{n, k}\right)^{\prime}\left(z_{j}\right)\right\|\right. \\
\quad & \left.\quad+\left(y_{j}-z_{j}\right) \operatorname{det}\left\|\psi_{n, k}\left(y_{p}\right), \theta_{n, k}\left(y_{j}, z_{j}\right)\right\|\right) \\
= & \left(z_{j}-y_{j}\right) \delta_{n, j}\left(y_{1}, \ldots, y_{m}\right) \rightrightarrows\left(z_{j}-y_{j}\right) \delta\left(y_{1}, \ldots, y_{m}\right) \\
= & \left(z_{j}-y_{j}\right)\left(\operatorname{det}\left\|\psi^{k}\left(y_{p}\right),\left(\psi^{k}\right)^{\prime}\left(z_{j}\right)\right\|_{0 \leqslant k \leqslant m, 1 \leqslant p \leqslant m}\right. \\
\quad & \left.\quad+\left(y_{j}-z_{j}\right) \operatorname{det}\left\|\psi^{k}\left(y_{p}\right), \theta_{k}\left(y_{j}, z_{j}\right)\right\|_{0 \leqslant k \leqslant m, 1 \leqslant p \leqslant m}\right),
\end{aligned}
$$

где $\left(y_{1}, \ldots, y_{m}\right) \in E_{1} \times \cdots \times E_{m}$, а смысл обозначений $\delta_{n, j}\left(y_{1}, \ldots, y_{m}\right)$ и $\delta\left(y_{1}, \ldots, y_{m}\right)$ ясен из контекста. Поскольку вьполняется условие

$$
\operatorname{det}\left\|\psi^{k}\left(z_{p}\right),\left(\psi^{k}\right)^{\prime}\left(z_{j}\right)\right\|_{0 \leqslant k \leqslant m, 1 \leqslant p \leqslant m} \neq 0, \quad 1 \leqslant j \leqslant m,
$$

то нетрудно увидеть, что для всякого достаточно малого $\varepsilon>0$ и при $y_{j} \in E_{j}=$ $E_{j}(\varepsilon)=\left\{z \in \mathbb{C}:\left|z-z_{j}\right| \leqslant \varepsilon\right\}$ имеет место соотношение $\delta_{j}\left(y_{1}, \ldots, y_{m}\right) \neq 0,1 \leqslant j \leqslant m$. Таким образом, имеем равенство

$$
\operatorname{det}\left\|\psi_{n, k}\left(y_{p}\right), \psi_{n, k}\left(z_{j}\right)\right\|_{\substack{0 \leqslant k \leqslant m \\ 1 \leqslant p \leqslant m}}=\left(z_{j}-y_{j}\right) \delta_{n, j}\left(y_{1}, \ldots, y_{m}\right), \quad 1 \leqslant j \leqslant m
$$

в котором

$$
\begin{aligned}
\delta_{n, j}\left(y_{1}, \ldots, y_{m}\right) \rightrightarrows & \delta_{j}\left(y_{1}, \ldots, y_{m}\right) \\
= & \operatorname{det}\left\|\psi^{k}\left(y_{p}\right),\left(\psi^{k}\right)^{\prime}\left(z_{j}\right)\right\|_{0 \leqslant k \leqslant m, 1 \leqslant p \leqslant m} \\
& +\left(y_{j}-z_{j}\right) \operatorname{det}\left\|\psi^{k}\left(y_{p}\right), \theta_{k}\left(y_{j}, z_{j}\right)\right\|_{0 \leqslant k \leqslant m, 1 \leqslant p \leqslant m} \neq 0,
\end{aligned}
$$

где $y_{j} \in E_{j}=E_{j}(\varepsilon), 1 \leqslant j \leqslant m$. Предполагаем далее, что $\varepsilon>0$ зафиксировано и удовлетворяет всем указанным вьше требованиям.

Выберем теперь $\delta \in(0,1)$ такое, что вьполняется условие $1 / \varkappa<\delta<\min \left\{\left|\psi\left(z_{j}\right)\right|\right.$, $1 \leqslant j \leqslant m\}$. Тогда, как уже отмечалось выше, $A_{n}(g)=o\left(\delta^{n}\right)$, и поэтому с учетом (3.41) система (3.40) приводится к виду

$$
\begin{aligned}
& \sum_{j=1}^{m}\left(z_{j}-y_{j}\right) d_{j} \cdot H_{n+s}\left(z_{j}\right) \delta_{n+s, j}\left(y_{1}, \ldots, y_{m}\right) \\
&=\operatorname{det}\left\|\psi_{n+s, k}\left(y_{p}\right), o\left(\delta^{n}\right)\right\|_{0 \leqslant k \leqslant m, 1 \leqslant p \leqslant m}, \quad 1 \leqslant s \leqslant m .
\end{aligned}
$$

Для определителя

$$
V_{n}\left(y_{1}, \ldots, y_{m}\right)=\operatorname{det}\left\|d_{j} H_{n+s}\left(z_{j}\right) \delta_{n+s, j}\left(y_{1}, \ldots, y_{m}\right)\right\|_{1 \leqslant s, j \leqslant m}
$$


имеем

$$
\begin{aligned}
\frac{V_{n}\left(y_{1}, \ldots, y_{m}\right)}{\prod_{j=1}^{m} H_{n}\left(z_{j}\right)} & =\widetilde{V}_{n}\left(y_{1}, \ldots, y_{m}\right) \rightrightarrows \widetilde{V}\left(y_{1}, \ldots, y_{m}\right) \\
& =\operatorname{det}\left\|d_{j} \psi^{s}\left(z_{j}\right) \delta_{j}\left(y_{1}, \ldots, y_{m}\right)\right\|_{1 \leqslant s, j \leqslant m} \\
& =\prod_{j=1}^{m} d_{j} \delta_{j}\left(y_{1}, \ldots, y_{m}\right) \operatorname{det}\left\|\psi^{s}\left(z_{j}\right)\right\|_{1 \leqslant s, j \leqslant m} \neq 0
\end{aligned}
$$

Поэтому для всех достаточно больших $n$ при $y_{j} \in E_{j}, 1 \leqslant j \leqslant m$, выполняется условие $V_{n}\left(y_{1}, \ldots, y_{m}\right) \neq 0$. Следовательно, система (3.42), а тем самьм и уравнение (3.34), эквивалентны системе уравнений

$$
z_{j}-y_{j}=\frac{V_{n, j}\left(y_{1}, \ldots, y_{m}\right)}{V_{n}\left(y_{1}, \ldots, y_{m}\right)}, \quad 1 \leqslant j \leqslant m,
$$

где $V_{n, j}\left(y_{1}, \ldots, y_{m}\right)$ - определитель, получающийся из определителя $V_{n}\left(y_{1}, \ldots, y_{m}\right)$ заменой $j$-го столбца на столбец из величин

$$
\operatorname{det}\left\|\psi_{n+s, k}\left(y_{p}\right), o\left(\delta^{n}\right)\right\|_{0 \leqslant k \leqslant m, 1 \leqslant p \leqslant m}, \quad 1 \leqslant s \leqslant m .
$$

С учетом выбора $\delta$ имеем: $o\left(\delta^{n}\right) / H_{n}\left(z_{j}\right) \rightarrow 0$ при $n \rightarrow \infty$. Тем самым получаем при $y_{j} \in E_{j}$ :

$$
\begin{gathered}
\widetilde{V}_{n, j}\left(y_{1}, \ldots, y_{m}\right)=V_{n, j}\left(y_{1}, \ldots, y_{m}\right) / \prod_{j=1}^{m} H_{n}\left(z_{j}\right) \rightrightarrows 0 \\
V_{n, j}\left(y_{1}, \ldots, y_{m}\right) / V_{n}\left(y_{1}, \ldots, y_{m}\right)=\widetilde{V}_{n, j}\left(y_{1}, \ldots, y_{m}\right) / \widetilde{V}_{n}\left(y_{1}, \ldots, y_{m}\right) \rightrightarrows 0 .
\end{gathered}
$$

Таким образом, в пределе при $n \rightarrow \infty$ система (3.43) переходит в систему

$$
z_{j}-y_{j}=0, \quad 1 \leqslant j \leqslant m
$$

которая имеет единственное решение $y_{j}=z_{j}, 1 \leqslant j \leqslant m$.

Поскольку функции, стоящие в правых частях уравнений (3.43), голоморфны по $y_{1}, y_{2}, \ldots, y_{m}$, то (см., например, [79; с. 287-288]) при каждом достаточно большом $n$ существует единственное решение системы (3.43) $y_{n, j} \in E_{j}=E_{j}(\varepsilon), 1 \leqslant j \leqslant m$, которое вместе с некоторьми $x_{n, j}$ является и решением исходной системы (3.33).

Так как доказанное утверждение справедливо для произвольного достаточно малого $\varepsilon>0$, то $y_{n, j} \rightarrow z_{j}$ при $n \rightarrow \infty$. Наконец, теперь уже легко увидеть, что величины $x_{n, j}, j=1, \ldots, m$, однозначно определяются по $y_{n, j}$, причем $x_{n, j} \rightarrow d_{j} \neq 0$ при $n \rightarrow \infty$.

Таким образом, лемма доказана (единственность решения будет установлена ниже).

3.3. В этом пункте мы в условиях леммы докажем существование обобщенных аппроксимаций Паде $F_{n, m}$ типа $(n, m)$ функции $f$ для всех достаточно больших $n$ и одновременно установим асимптотику поведения конечных полюсов функции $F_{n, m}$ при $n \rightarrow \infty$. 
В соответствии с утверждением леммы, заменяя в системе (3.33) $n$ на $n-m$, найдем, что полученная система для каждого достаточно большого $n$ имеет решение $x_{n-m, j}$, $y_{n-m, j}, 1 \leqslant j \leqslant m$, причем

$$
\lim _{n \rightarrow \infty} y_{n-m, j}=z_{j}, \quad 1 \leqslant j \leqslant m
$$

Для рациональной функции

$$
r_{n, m}(z)=-\sum_{j=1}^{m} \frac{x_{n-m, j}}{z-y_{n-m, j}} \in \mathscr{R}_{m-1, m}
$$

имеем

$$
A_{k}\left(r_{n, m}\right)=\sum_{j=1}^{m} x_{n-m, j} H_{k}\left(y_{n-m, j}\right)=A_{k}(f), \quad n-m+1 \leqslant k \leqslant n+m .
$$

Подберем теперь полином $p_{n} \in \mathbb{C}[z], \operatorname{deg} p_{n} \leqslant n-m$, так, чтобы вьполнялись соотношения

$$
A_{k}\left(p_{n}\right)=A_{k}(f)-A_{k}\left(R_{n, m}\right), \quad k=0,1, \ldots, n-m .
$$

Ясно, что при вьполнении всех этих условий функция $r_{n, m}+p_{n}$ является обобщенной аппроксимацией Паде типа $(n, m)$ функции $f$, т.е. имеем

$$
F_{n, m}(z)=-\sum_{j=1}^{m} \frac{x_{n-m, j}}{z-y_{n-m, j}}+p_{n}(z)
$$

Наконец, так как при любом фиксированном $n$ каждому решению системы (3.33) по приведенной схеме можно однозначньм образом поставить в соответствие обобщенную аппроксимацию Паде $F_{n, m}$ функции $f$, то в силу единственности такой рациональной апшроксимации при каждом достаточно большом $n$ система (3.33) имеет единственное решение.

3.4. Итак, в соответствии с результатом предыдущего пункта для всякого достаточно большого $n$ рациональная функщия $F_{n, m}$ допускает представление

$$
F_{n, m}(z)=\sum_{j=1}^{m} \frac{d_{n, j}}{z-z_{n, j}}+p_{n}(z)
$$

где $\operatorname{deg} p_{n} \leqslant n-m, d_{n, j}=-x_{n-m, j}, z_{n, j}=y_{n-m, j}$ и $z_{n, j} \rightarrow z_{j}, 1 \leqslant j \leqslant m$.

Как уже отмечалось выше, функция $f$ представляется в виде

$$
f(z)=-\sum_{j=1}^{m} \frac{d_{j}}{z-z_{j}}+g(z)
$$

где $d_{j} \neq 0$ и $g \in \mathscr{H}\left(D_{\varkappa}\right)$. Следовательно, для $k \geqslant n-m+1$ имеем

$$
b_{k}^{(n, m)}=A_{k}\left(f-F_{n, m}\right)=\sum_{j=1}^{m}\left(d_{j} H_{k}\left(z_{j}\right)+d_{n, j} H_{k}\left(z_{n, j}\right)\right)+A_{k}(g) .
$$


В силу определения обобщенной аппроксимации Паде функции $f$ вьполняются условия $A_{k}\left(f-F_{n, m}\right)=0, k=0,1, \ldots, n+m$. Рассмотрим последние $2 m$ из этих условий. В силу формулы (3.44) эти $2 m$ соотношений при $k=n-m+1, \ldots, n+m$ можно записать в виде системы

$$
\sum_{j=1}^{m}\left(d_{j} H_{k}\left(z_{j}\right)+d_{n, j} H_{k}\left(z_{n, j}\right)\right)+A_{k}(g)=0, \quad k=n-m+1, \ldots, n+m
$$

Эти $2 m$ уравнений связывают между собой $2 m$ коэффициентов $d_{j}$ и $d_{n, j}$ посредством величин $H_{k}\left(z_{j}\right), H_{k}\left(z_{n, j}\right)$ и $A_{k}(g)$.

Наша ближайшая задача такова: исходя из системы (3.45), выразить $d_{j}$ и $d_{n, j}$ через $H_{k}\left(z_{j}\right), H_{k}\left(z_{n, j}\right)$ и $A_{k}(g)$, подставить полученные значения в формулу $(3.44)$ при $k=n+m+1, \ldots, n+4 m$ и получить оценку для коэффициентов $A_{k}\left(f-F_{n, m}\right)$, которая будет использована в дальнейшем при доказательстве остальных утверждений теоремы 6. Эта оценка имеет вид $\left|A_{k}\left(f-F_{n, m}\right)\right| \leqslant c(m, q) \cdot q^{n}, k=n+m+1, \ldots, n+4 m$, где $q<1, q \in(1 / \varkappa, 1), c(m, q)$ - константа, зависящая от $m$ и $q$, но не зависяшая от $n$.

Система $2 m$ уравнений (3.45) относительно $2 m$ величин $d_{j}, d_{n, j}, 1 \leqslant j \leqslant m$, зависит от точек $z_{j}$ и $z_{n, j}$. Не ограничивая общности нижеследующих рассуждений, можно считать, что для всех достаточно больших $n$ (только такие $n$ и будут рассматриваться в дальнейшем) и для $j=1,2, \ldots, m$ вьполняются условия $z_{n, j} \neq z_{\ell}$. Действительно, пусть $\left\{M_{s}\right\}-$ совокупность $2^{m}$ различных подмножеств множества $\{1,2, \ldots, m\}$. Тогда в обшем случае все множество натуральных чисел $\mathbb{N}$ представляется в виде объединения $2^{m}$ непересекающихся подмножеств

$$
\Lambda_{s}=\Lambda\left(M_{s}\right)=\left\{n \in \mathbb{N}: z_{n, j}=z_{\ell}, j \in M_{s}\right\}
$$

И для получения требуемой оценки величины $A_{k}\left(f-F_{n, m}\right)$ все последующие рассуждения надо проводить отдельно для каждого множества $\Lambda_{s}, s=1,2, \ldots, 2^{m}$, с соответствующими небольшими изменениями.

Итак, по сделанному предположению $z_{n, j} \neq z_{\ell}, 1 \leqslant j \leqslant m$. Покажем теперь, что при этом условии для всех достаточно больших $n$

$$
\Delta_{n}=\operatorname{det}\left\|H_{\nu+k}\left(z_{j}\right), H_{\nu+k}\left(z_{n, j}\right)\right\|_{1 \leqslant k \leqslant 2 m, 1 \leqslant j \leqslant m} \neq 0,
$$

где мы положили $\nu=n-m$.

Действительно, имеем равенства

$$
\begin{gathered}
\Delta_{n}=\prod_{j=1}^{m} H_{\nu}\left(z_{j}\right) H_{\nu}\left(z_{n, j}\right) \operatorname{det}\left\|\psi_{\nu, k}\left(z_{j}\right), \psi_{\nu, k}\left(z_{n, j}\right)\right\| \\
\operatorname{det}\left\|\psi_{\nu, k}\left(z_{j}\right), \psi_{\nu, k}\left(z_{n, j}\right)\right\|=\prod_{j=1}^{m}\left(z_{j}-z_{n, j}\right) \operatorname{det}\left\|\frac{\psi_{\nu, k}\left(z_{j}\right)-\psi_{\nu, k}\left(z_{n, j}\right)}{z_{j}-z_{n, j}}, \psi_{\nu, k}\left(z_{n, j}\right)\right\| \\
=\prod_{j=1}^{m}\left(z_{j}-z_{n, j}\right) \cdot \widetilde{\Delta}_{n}
\end{gathered}
$$


и, кроме того, предельное соотношение

$$
\lim _{n \rightarrow \infty} \widetilde{\Delta}_{n}=\widetilde{\Delta}=\operatorname{det}\left\|\left(\psi^{k}\right)^{\prime}\left(z_{j}\right), \psi^{k}\left(z_{j}\right)\right\|_{1 \leqslant k \leqslant 2 m, 1 \leqslant j \leqslant m} \neq 0 .
$$

Следовательно, $\widetilde{\Delta}_{n} \neq 0$ при достаточно больших $n$ и при тех же $n$ имеем

$$
\Delta_{n}=\prod_{j=1}^{m} H_{\nu}\left(z_{j}\right) H_{\nu}\left(z_{n, j}\right)\left(z_{j}-z_{n, j}\right) \cdot \widetilde{\Delta}_{n} \neq 0
$$

При этом условии система (3.45) имеет "решение" $d_{j}=\Delta_{n}(2 j-1) / \Delta_{n}, d_{n, j}=$ $\Delta(2 j) / \Delta_{n}, 1 \leqslant s \leqslant m$, где $\Delta_{n}(s)$ - определитель, полученный из определителя $\Delta_{n}$ заменой $s$-го столбца на столбец величин $A_{\nu+k}(-g), k=1,2, \ldots, 2 m$, т.е. $\Delta_{n}(s)=$ $\sum_{k=1}^{2 m}(-1)^{k+s} A_{\nu+k}(-g) M_{n}(k, s)$, где $M_{n}(k, s)$ - минор элемента с номерами $k, s$ в матрице системы (3.45). Следовательно, справедливы формулы

$$
\begin{gathered}
d_{j}=-\frac{1}{\Delta_{n}} \sum_{k=1}^{2 m}(-1)^{2 j-1+k} A_{\nu+k}(g) M_{n}(k, 2 j-1), \\
d_{n, j}=-\frac{1}{\Delta_{n}} \sum_{k=1}^{2 m}(-1)^{2 j+k} A_{\nu+k}(g) M_{n}(k, 2 j), \quad 1 \leqslant j \leqslant m .
\end{gathered}
$$

Подставляя (3.47) в (3.44), находим последовательно

$$
\begin{aligned}
A_{\nu+s}\left(f-F_{n, m}\right)= & -\frac{1}{\Delta_{n}} \sum_{j=1}^{m} \sum_{k=1}^{2 m}\left((-1)^{2 j-1+k} A_{\nu+k}(g) M_{n}(k, 2 j-1) H_{\nu+s}\left(z_{j}\right)\right. \\
& \left.+(-1)^{2 j+k} A_{\nu+k}(g) M_{n}(k, 2 j) H_{\nu+s}\left(z_{n, j}\right)\right)+A_{\nu+s}(g) \\
= & -\frac{1}{\Delta_{n}} \sum_{k=1}^{2 m}\left(\sum_{j=1}^{m}(-1)^{2 j-1+k} M_{n}(k, 2 j-1) H_{\nu+s}\left(z_{j}\right)\right. \\
& \left.+(-1)^{2 j+k} M_{n}(k, 2 j) H_{\nu+s}\left(z_{n, j}\right)\right) A_{\nu+k}(g)+A_{\nu+s}(g) \\
= & -\frac{1}{\Delta_{n}} \sum_{k=1}^{2 m} \Delta_{n}(k, s) A_{\nu+k}(g)+A_{\nu+s}(g) .
\end{aligned}
$$

Здесь $s=2 m+1, \ldots, 4 m$, а $\Delta_{n}(k, s)$ - определитель, получаюшийся из $\Delta_{n}$ заменой $k$-й строки, состоящей из $H_{\nu+k}\left(z_{j}\right), H_{\nu+k}\left(z_{n, j}\right), 1 \leqslant j \leqslant m$, на строку из $H_{\nu+s}\left(z_{j}\right)$, $H_{\nu+s}\left(z_{n, j}\right), 1 \leqslant j \leqslant m$. Далее, нетрудно доказать, что

$$
\begin{gathered}
\Delta_{n}(k, s)=\prod_{j=1}^{m}\left(H_{\nu}\left(z_{j}\right) H_{\nu}\left(z_{n, j}\right)\left(z_{j}-z_{n, j}\right)\right) \widetilde{\Delta}_{n}(k, s), \\
\lim _{n \rightarrow \infty} \widetilde{\Delta}_{n}(k, s)=\widetilde{\Delta}(k, s), \quad s=2 m+1, \ldots, 4 m,
\end{gathered}
$$


где определители $\widetilde{\Delta}_{n}(k, s)$ и $\widetilde{\Delta}(k, s)$ получаются по аналогичному правилу соответственно из определителей

$$
\begin{aligned}
\widetilde{\Delta}_{n} & =\operatorname{det}\left\|\frac{\psi_{\nu, p}\left(z_{j}\right)-\psi_{\nu, p}\left(z_{n, j}\right)}{z_{j}-z_{n, j}}, \psi_{\nu, p}\left(z_{n, j}\right)\right\|_{1 \leqslant p \leqslant 2 m, 1 \leqslant j \leqslant m}, \\
\widetilde{\Delta} & =\operatorname{det}\left\|\left(\psi^{p}\right)^{\prime}\left(z_{j}\right), \psi^{p}\left(z_{j}\right)\right\|_{1 \leqslant p \leqslant 2 m, 1 \leqslant j \leqslant m} \neq 0 .
\end{aligned}
$$

Таким образом, при $k=1,2, \ldots, 2 m$ и $s=2 m+1, \ldots, 4 m$ имеем предельное соотношение

$$
\lim _{n \rightarrow \infty} \frac{\Delta_{n}(k, s)}{\Delta_{n}}=\lim _{n \rightarrow \infty} \frac{\widetilde{\Delta}_{n}(k, s)}{\widetilde{\Delta}_{n}}=\frac{\widetilde{\Delta}(k, s)}{\widetilde{\Delta}},
$$

из которого следует неравенство

$$
\left|\frac{\Delta_{n}(k, s)}{\Delta_{n}}\right| \leqslant c_{1}<\infty, \quad k=1, \ldots, 2 m, \quad s=2 m+1, \ldots, 4 m,
$$

где $c_{1}$ - константа, не зависящая от $n$ (ниже через $c_{2}, c_{3}, \ldots$ обозначаются константы, также не зависящие от $n$ ).

Как уже отмечалось вьше, $A_{\ell}(g)=o\left(q^{\ell}\right)$ при $\ell \rightarrow \infty$ для любого $q \in(1 / \varkappa, 1)$, т.е. имеем

$$
\left|A_{\ell}(g)\right| \leqslant c_{2} q^{\ell}, \quad \ell=0,1,2, \ldots, \quad q \in(1 / \varkappa, 1) .
$$

Напомним, что для коэффициентов Фурье функции $f-F_{n, m}$ вьше было получено соотношение

$$
A_{\nu+s}\left(f-F_{n, m}\right)=-\sum_{k=1}^{2 m} \frac{\Delta_{n}(k, s)}{\Delta_{n}} A_{\nu+s}(g)+A_{\nu+s}(g),
$$

из которого теперь в силу неравенств (3.48) и (3.49) находим

$$
\left|A_{\nu+s}\left(f-F_{n, m}\right)\right| \leqslant c_{1} c_{2} \sum_{k=1}^{2 m} q^{\nu+k}+c_{2} q^{\nu+s} \leqslant c_{3} q^{n}, \quad s=2 m+1, \ldots, 4 m .
$$

Таким образом, для коэффициентов Фурье функции $f-F_{n, m}$ получена необходимая оценка

$$
\left|b_{k}^{(n, m)}\right|=\left|A_{k}\left(f-F_{n, m}\right)\right| \leqslant c_{3} q^{n}, \quad k=n+m+1, \ldots, n+3 m .
$$

3.5. Докажем теперь основные утверждения теоремы 6.

Положим

$$
\omega_{m}(z)=\prod_{j=1}^{m}\left(z-z_{j}\right), \quad \omega_{n, m}(z)=\prod_{j=1}^{m}\left(z-z_{n, j}\right) .
$$

Умножая обе части разложения (3.32) на многочлен $\omega_{m} \omega_{n, m}$, получаем

$$
\begin{aligned}
& \left(\omega_{n, m} f \omega_{m}-\omega_{m} F_{n, m} \omega_{n, m}\right)(z) \\
& \quad=\sum_{k=n+m+1}^{\infty} b_{k}^{(n, m)} P_{k}(z) \omega_{m}(z) \omega_{n, m}(z), \quad z \in D_{0}\left(f-F_{n, m}\right) .
\end{aligned}
$$


Учитьвая теперь, что $f \omega_{m} \in \mathscr{H}\left(D_{\varkappa}\right)$, a $\operatorname{deg} \omega_{m} F_{n, m} \omega_{n, m} \leqslant n+2 m$, при $z \in D_{\varkappa}$ находим разложение

$$
\begin{aligned}
\left(\omega_{n, m} f \omega_{m}-\omega_{m} F_{n, m} \omega_{n, m}\right)(z) & =\sum_{s=0}^{\infty} b_{n, s} P_{s}(z) \\
& =\sum_{s=0}^{n+2 m} b_{n, s} P_{s}(z)+\sum_{s=n+2 m+1}^{\infty} b_{n, s} P_{s}(z),
\end{aligned}
$$

для коэффициентов которого при $s \geqslant n+2 m+1$ справедлива формула

$$
b_{n, s}=\frac{1}{\pi i} \int_{\Gamma_{\rho}} \omega_{n, m}(t) f(t) \omega_{m}(t) H_{s}(t) d t
$$

где $\rho \in(1, \varkappa)$. В соответствии с разложением $(3.51)$ и условием

$$
\left(P_{k} \omega_{n, m} \omega_{m}, P_{s}\right)=0, \quad s>k+2 m \text { или } k>s+2 m,
$$

при $s=n-m+1, \ldots, n+2 m$ имеем

$$
b_{n, s}=\sum_{k=n+m+1}^{\infty} b_{k}^{(n, m)}\left(P_{k} \omega_{n, m} \omega_{m}, P_{s}\right)=\sum_{k=s-2 m}^{s+2 m} b_{k}^{(n, m)}\left(P_{k} \omega_{n, m} \omega_{m}, P_{s}\right) .
$$

Кроме того, $b_{s, n}=0$ при $s=0,1, \ldots, n-m$. С помощью полученных формул нетрудно оценить коэффициенты $\left\{b_{n, s}\right\}$. Используя оценку (3.50) и неравенство

$$
\begin{aligned}
\left|\left(P_{k} \omega_{n, m} \omega_{m}, P_{s}\right)\right| & \leqslant \max _{-1 \leqslant x \leqslant 1}\left|\omega_{n, m}(x) \omega_{m}(x)\right|\left(P_{k}, P_{k}\right) \cdot\left(P_{s}, P_{s}\right) \\
& =\max _{-1 \leqslant x \leqslant 1}\left|\omega_{n, m}(x) \omega_{m}(x)\right| \leqslant c_{4},
\end{aligned}
$$

находим

$$
\left|b_{n, s}\right| \leqslant 4 m c_{4} c_{3} q^{n}=c_{5} q^{n}, \quad s=n-m+1, \ldots, n+2 m .
$$

Аналогично из (3.52) получаем

$$
\left|b_{n, s}\right| \leqslant c_{6} q^{s}, \quad s \geqslant n+2 m+1 .
$$

С другой стороны, из соотношения (3.6) при $z \in \bar{D}_{\rho}, \rho \in(1, \varkappa)$, имеем

$$
\left|P_{s}(z)\right| \leqslant c_{7}(\rho+\varepsilon)^{s}, \quad s=0,1,2, \ldots,
$$

где $\varepsilon>0$ выбрано так, что $\rho+\varepsilon<\varkappa-\varepsilon$, т.е. $\theta=(\rho+\varepsilon) /(\varkappa-\varepsilon)<1$. Зафиксируем это $\varepsilon$ и положим $q=1 /(\varkappa-\varepsilon)$. Тогда с помошью $(3.54)$ и (3.56) имеем

$$
\left|\sum_{s=n-m+1}^{n+2 m} b_{n, s} P_{s}(z)\right| \leqslant c_{5} q^{n} \sum_{s=n-m+1}^{n+2 m}(\rho+\varepsilon)^{s} \leqslant c_{8} q^{n}(\rho+\varepsilon)^{n}=c_{8} \theta^{n} .
$$


Аналогично с помощью неравенств (3.55) и (3.56) находим

$$
\left|\sum_{s=n+m+1}^{\infty} b_{n, s} P_{s}(z)\right| \leqslant c_{6} c_{7} \sum_{s=n+m+1}^{\infty} q^{s}(\rho+\varepsilon)^{s} \leqslant c_{9} \theta^{n} .
$$

Из этих двух оценок вытекает неравенство

$$
\left|\left(\omega_{n, m} f \omega_{m}-\omega_{m} F_{n, m} \omega_{n, m}\right)(z)\right| \leqslant c_{10} \theta^{n},
$$

где $z \in \bar{D}_{\rho}, \rho \in(1, \varkappa), c_{10}=c_{10}(\rho, \varepsilon)$.

Следовательно, для любого компакта $K \subset D_{\varkappa}$ при $z \in K$ и всех достаточно больших $n$ имеем оценку

$$
\left|\left(f-F_{n, m}\right)(z)\right| \leqslant \frac{c_{11} \theta^{n}}{\left|\omega_{m}(z) \omega_{n, m}\right|},
$$

где $\theta=(\rho(K)+\varepsilon) /(\varkappa-\varepsilon)<1, c_{11}=c_{11}(\varepsilon, K)$. Из этой оценки, учитывая асимптотику поведения нулей полинома $\omega_{n, m}$, получаем, что последовательность $\left\{F_{n, m}\right\} \rightarrow f$ при $n \rightarrow \infty$ равномерно в сферической метрике на $K$. Кроме того, имеет место неравенство

$$
\varlimsup_{n \rightarrow \infty}\left|\left(f-F_{n, m}\right)(z)\right|^{1 / n} \leqslant \frac{|\varphi(z)|}{\rho_{m}}<1 .
$$

Таким образом, теорема 6 полностью доказана.

\section{СПИСОК ЛИТЕРАТУРЫ}

[1] А. А. Гончар, Е. А. Рахманов. Равновесные распределения и скорость рациональной аппроксимации аналитической функции // Матем. сб. 1987. Т. 134 (176). № 3. С. 306-352.

[2] Г. Шталь. Наилучшие равномерные рациональные аппроксимации $|x|$ на $[-1,1] / /$ Матем. сб. 1992. Т. 183. № 8. С. 85-118.

[3] В.Н. Сорокин. Аппроксимации Эрмита-Паде для систем Никишина и иррациональность $\zeta(3) / /$ УМН. 1994. Т. 49. № 2. С. 167-168.

[4] В.В.Зудилин. Об оценках снизу многочленов от значений некоторых целых функций // Матем. сб. 1996. Т. 187. № 12. С. 57-86.

[5] А. А. Гончар, Н. Н. Новикова, Г. М. Хенкин. Многоточечные аппроксимации Паде в обратной задаче Штурма-Лиувилля // Матем. сб. 1991. Т. 182. № 8. С. 1118-1128.

[6] Е. М. Никишин. Дискретньй оператор Штурма-Лиувилля и некоторые задачи теории функций // Тр. семин. им. И. Г. Петровского. 1984. № 10. С. 3-77.

[7] В. А. Калягин. Аппроксимации Эрмита-Паде и спектральный анализ несимметричных разностных операторов // Матем. сб. 1994. Т. 185. №6. С. 79-100.

[8] A. I. Aptekarev, V.A. Kaliaguine, W. Van Assche. Criterion for the resolvent set of nonsymmetric tridiagonal operators // Proc. Amer. Math. Soc. 1995. V. 123. № 8 . P. 2423-2430.

[9] J. Nuttall. Sets of minimum capacity, Padé approximants and the bubble problem // Bifurcation Phenomena in Mathematical Physics and Related Topics / ed. C. Bardos and D. Bessis. Dordrecht: Reidel, 1980. P. 185-201.

[10] I. V. Andrianov. Application of Padé-approximants in perturbation methods // Adv. Mech. 1991. V. 14. № 2. P. 3-25.

[11] M. B. Dadfar, J.F. Geer, C.M. Andersen. Perturbation analysis of the limit cycle of the free van der Pol equation // SIAM J. Appl. Math. 1984. V. 44. № 5. P. 881-895.

[12] C. M. Andersen, J. F. Geer. Power series expansions for the frequency and period of the limit cycle of the van der Pol equation // SIAM J. Appl. Math. 1982. V. 42. № 3. P. 678-693. 
[13] Дж. Бейкер, П. Грейвс-Моррис. Аппроксимации Паде. М.: Мир, 1986.

[14] R. de Montessus de Ballore. Sur les fractions continues algébriques // Bull. Soc. Math. France. 1902. V. 30. P. 28-36.

[15] J. Hadamard. Essai sur l'étude des fonctions données par leur développement de Taylor // J. Math. 1892. V. 8. № 4. P. 101-186.

[16] А. А. Гончар. Полюсы строк таблицы Паде и мероморфное продолжение функций // Матем. сб. 1981. Т. 115 (157). № 4. С. 590-613.

[17] E. Fabry. Sur les points singuliers d'une fonction donnée par son développement de Taylor // Ann. École Norm. Sup. Paris. 1896. V. 13. № 3. P. 367-399.

[18] Л. Бибербах. Аналитическое продолжение. М.: Наука, 1967.

[19] А. А. Марков. Deux démonstrations de la convergence de certaines fractions continues // Acta Math. 1895. V. 19. Р. 93-104. (Два доказательства сходимости некоторых непрерьвных дробей // Избранные труды по теории непрерьвных дробей и теории функций, наименее уклоняющихся от нуля. М.: Гостехиздат, 1948. С. 106-119.)

[20] Н. У. Аракелян. Об эффективном аналитическом продолжении степенных рядов // Матем. сб. 1984. Т. 124 (166). № 1. С. 24-44.

[21] Н. У. Аракелян, В.А. Мартиросян. Локализация особенностей степенных рядов на границе круга сходимости // Изв. АН Арм. ССР. Матем. 1987. Т. 22. № 1. С. 3-21.

[22] Н. У. Аракелян, В.А.Мартиросян. Локализация особенностей степенных рядов на границе круга сходимости. II // Изв. АН Арм. ССР. Матем. 1988. Т. 23. № 2. С. 123-137.

[23] А. В. Яврян. О распределении особенностей и скорости суммирования степенных рядов // Дисс. . . . канд. физ.-матем. наук. Ереван: Ереванский государственньй ун-т, 1997.

[24] С. П. Суетин. О полюсах $m$-й строки таблицы Паде // Матем. сб. 1983. Т. 120 (162). № 4. C. $500-504$.

[25] С. П. Суетин. Об одной обратной задаче для $m$-й строки таблицы Паде // Матем. сб. 1984. T. 124 (166). № 2. C. 238-250.

[26] В. И. Буслаев, А. А. Гончар, С. П. Суетин. О сходимости подпоследовательностей m-й строки таблицы Паде // Матем. сб. 1983. Т. 120 (162). № 4. С. 540-545.

[27] В. В. Вавилов, В.А.Прохоров, С. П. Суетин. Полюсы $m$-й строки таблицы Паде и особые точки функции // Матем. сб. 1985. Т. 122 (164). №4 (12). С. 475-480.

[28] С. П. Суетин. Асимптотика полиномов Ахиезера и равномерная сходимость аппроксимаций Паде для гиперэллиптических функций // УМН. 1998. Т. 53. №6. С. 267-268.

[29] С. П. Суетин. О равномерной сходимости диагональных аппроксимаций Паде для гиперэллиптических функций // Матем. сб. 2000. Т. 191. № 9. С. 81-114.

[30] П. Л. Чебышёв. О непрерьвных дробях // Ученые записки Имп. Академии Наук. 1855. T. III. С. 636-664. (Полное собрание сочинений. Т. II. М.-Л.: Изд-во АН СССР, 1948. C. 103-126.)

[31] А. А. Гончар. О сходимости аппроксимаций Паде для некоторых классов мероморфных функций // Матем. сб. 1975. Т. 97 (139). № 4 (8). С. 607-629.

[32] Е.А. Рахманов. О сходимости диагоналшных аппроксимаций Паде // Матем. сб. 1977. T. 104 (146). № 2. C. 271-291.

[33] H. Stahl. Conjectures around Baker-Gammel-Wills conjecture // Constr. Approx. 1997. V. 13. № 2. P. 287-292.

[34] H. Stahl. Orthogonal polynomials with complex-valued weight function. I; II // Constr. Approx. 1986. V. 2. P. 225-240; 241-251.

[35] J. Nuttall, R.S. Singh. Orthogonal polynomials and Padé approximants associated with a system of arcs // J. Approx. Theory. 1977. V. 21. P. 1-42.

[36] S. Dumas. Sur le développement des fonctions elliptiques en fractions continues. Thesis. Zürich, 1908.

[37] С. Н. Бернштейн. О многочленах, ортогоналшных на конечном отрезке // С. Н. Бернштейн. Собрание сочинений. Т. II. М.: АН СССР, 1952. С. 7-106.

[38] Н.И. Ахиезер. Об ортогональных многочленах на нескольких интервалах // Докл. АН CCCP. 1960. T. 134. № 1. C. 9-12. 
[39] J. Nuttall. Asymptotics of diagonal Hermite-Padé polynomials // J. Approx. Theory. 1984. V. 42. P. 299-386.

[40] N. H. Li, J. Nuttall. An extension of a singular integral equation approach to Padé polynomial asymptotics // Approximation Theory VI: Proceedings of the 6th International Symposium. V. 1 / ed. C. Chui, L. Schumaker, and J. Ward. New York: Academic Press, 1989. P. 391-394.

[41] J. Nuttall. Padé polynomial asymptotics from a singular integral equation // Constr. Approx. 1990. V. 6. № 2. P. 157-166.

[42] N. H. Li. Strong asymptotics of Padé polynomials. Thesis. Western Ontario, London: University of Western Ontario, 1991.

[43] H. Stahl. Diagonal Padé approximants to hyperelliptic functions // Ann. Fac. Sci. Toulouse Math. (6). Spec. Iss. 1996. P. 121-193.

[44] J. T. Holdeman (Jr.). A method for the approximation of functions defined by formal series expansions in orthogonal polynomials // Math. Comput. 1969. V. 23. № 106. P. 275-287.

[45] J. Fleischer. Nonlinear Padé approximants for Legendre series // J. Math. Phys. 1973. V. 14. № 2. P. 246-248.

[46] С. П. Суетин. О сходимости рациональных аппроксимаций полиномиальных разложений в областях мероморфности заданной функции // Матем. сб. 1978. Т. 105 (147). № 3. C. $413-430$.

[47] С. П. Суетин. Обратные теоремы об обобщенных аппроксимациях Паде // Матем. сб. 1979. T. 109 (151). № 4. C. 629-646.

[48] С. П. Суетин. О теореме Монтессу де Болора для нелинейных аппроксимаций Паде ортогональных разложений и рядов Фабера // Докл. АН СССР. 1980. Т. 253. № 6. С. 1322-1325.

[49] С. П. Суетин. О теореме Монтессу де Болора для рациональных аппроксимаций ортогональных разложений // Матем. сб. 1981. Т. 114 (156). № 3. С. 451-464.

[50] С. П. Суетин. Вопросы сходимости аппроксимаций Паде-Фабера // Дисс. ... канд. физ.-матем. наук. М.: МГУ, 1981.

[51] А. А. Гончар, Е.А. Рахманов, С. П. Суетин. О сходимости аппроксимаций Паде ортогональных разложений // Тр. МИАН. 1991. Т. 200. С. 136-146.

[52] A. A. Gonchar, E. A. Rakhmanov, S. P. Suetin. On the rate of convergence of Padé approximants of orthogonal expansions // Progress in Approximation Theory / ed. A. A. Gonchar et al. New York: Springer-Verlag, 1992. P. 169-190. (Springer Ser. Comput. Math. V. 19.)

[53] С. П. Суетин. Об асимптотике знаменателей диагональных аппроксимаций Паде ортогональных разложений // Докл. РАН. 1997. Т. 356. № 6. С. 744-746.

[54] S. Agmon. Sur les series de Dirichlet // Ann. Sci. École Norm. Sup. 1949. T. 66. P. 263-310.

[55] С. Мандельбройт. Ряды Дирихле. Принципы и методы. М.: Мир, 1973.

[56] П. Л. Чебышёв. Полное собрание сочинений. Т. II, III. М.-Л.: Изд-во АН СССР, 1948.

[57] А. А. Марков. Избранные труды по теории непрерьвных дробей и теории функций, наименее уклоняющихся от нуля. М.: Гостехиздат, 1948.

[58] H. Widom. Extremal polynomials associated with a system of curves in the complex plane // Adv. Math. 1969. V. 3. P. 127-232.

[59] А. И. Аптекарев. Асимптотические свойства многочленов, ортогональных на системе контуров, и периодические движения цепочек Тода // Матем. сб. 1984. Т. 125 (167). № 2 (10). C. $231-258$.

[60] Н. И. Ахиезер, Ю. Я. Томчук. К теории ортогональных многочленов на нескольких интервалах // Докл. АН СССР. 1961. Т. 138. № 4. С. 743-746.

[61] Н. И. Ахиезер. Континуальные аналоги ортогоналшных многочленов на системе интервалов // Докл. АН СССР. 1961. Т. 141. № 2. С. 263-266.

[62] H. Stahl. Extremal domains associated with an analytic function. I; II // Complex Variables Theory Appl. 1985. V. 4. P. 311-324; 325-338.

[63] H. Stahl. The structure of extremal domains associated with an analytic function // Complex Variables Theory Appl. 1985. V. 4. P. 339-354.

[64] Э. И. Зверович. Краевые задачи теории аналитических функций в гёльдеровских классах на римановых поверхностях // УМН. 1971. Т. 26. №1. С. 113-180. 
[65] F. Peherstorfer. Minimal polynomials on several intervals with respect to the maximum norm. A survey // Complex Methods in Approximation Theory / ed. A. Martínez Finkelshtein et al. Almería: Universidad de Almería, 1997. P. 137-160.

[66] Б. А. Дубровин. Тэта-функции и нелинейные уравнения // УМН. 1981. Т. 36. № 2. C. $11-80$.

[67] Дж. Спрингер. Введение в теорию римановых поверхностей. М.: ИЛ, 1960.

[68] О. Форстер. Римановы поверхности. М.: Мир, 1980.

[69] A. Krazer. Lehrbuch der Thetafunktionen. New York: Chelsea, 1970.

[70] Ф. Д. Гахов. Краевые задачи. М.: Физматгиз, 1977.

[71] А. Зигмунд. Тригонометрические ряды. Т. І. М.: Мир, 1965.

[72] E. B. Saff. An extension of Montessus de Ballore's theorem on the convergence of interpolating rational functions // J. Approx. Theory. 1972. V. 6. P. 63-67.

[73] А. А. Гончар. О сходимости обобщенных аппроксимаций Паде мероморфных функций // Матем. сб. 1975. Т. 98 (140). № 4 (12). С. 564-577.

[74] Е. А. Рахманов. Об асимптотике отношения ортогональных многочленов // Матем. сб. 1977. T. 103 (145). № 2 (6). C. 237-252.

[75] Е. А. Рахманов. Об асимптотике отношения ортогональных многочленов. II // Матем. сб. 1982. Т. 118 (160). № 1 (5). С. 104-117.

[76] Г. Сегё. Ортогональные многочлены. М.: Физматгиз, 1962.

[77] Г. М. Голузин. Геометрическая теория функций комплексного переменного. М.: Наука, 1966.

[78] В. В. Вавилов. О сходимости аппроксимаций Паде мероморфных функций // Матем. сб. 1976. T. 101 (143). № 1 (9). C. 44-56.

[79] Б. В. Шабат. Введение в комплексньй анализ. Ч. ІІ. М.: Наука, 1976.

Математический институт

Поступила в редакцию

им. В. А. Стеклова РАН

15.10 .2001

E-mail: suetin@mi.ras.ru 